\title{
HOCHAUFLÖSENDE BILDGEBUNG UND NEXAFS-SPEKTROSKOPIE MIT WEICHER RÖNTGENSTRAHLUNG AUS LASERINDUZIERTEN PLASMEN
}

\author{
Dissertation \\ zur Erlangung des mathematisch-naturwissenschaftlichen Doktorgrades \\ „Doctor rerum naturalium" \\ der Georg-August-Universität Göttingen \\ im Promotionsprogramm ProPhys \\ der Georg-August University School of Science (GAUSS) \\ vorgelegt von \\ MATTHIAS MÜLLER \\ aus Karl-Marx-Stadt (heute Chemnitz)
}

Göttingen, 2018 
Betreuungsausschuss

Professor Dr. Tim Salditt

Institut für Röntgenphysik

Georg-August-Universität Göttingen

Dr. Klaus Mann

Abteilung „Optik / Kurze Wellenlängen“

Laser-Laboratorium Göttingen e.V.

Mitglieder der Prüfungskommission

Referent: Professor Dr. Tim Salditt

Korreferent: Dr. Klaus Mann

Weitere Mitglieder der Prüfungskommission

PD Dr. Alexander Egner

Laser-Laboratorium Göttingen e.V.

Professor Dr. Arnulf Quadt

II. Physikalisches Institut

Georg-August-Universität Göttingen

PD Dr. Jürgen Niemeyer

Pflanzenernährung \& Ertragsphysiologie

Georg-August-Universität Göttingen

Professor Dr. Claus Ropers

IV. Physikalisches Institut

Georg-August-Universität Göttingen

Tag der mündlichen Prüfung: 22.10.2018 
Soft x-ray microscopy and absorption spectroscopy are extremely useful tools for high-resolution imaging and chemical analysis of samples in various scientific fields. However, due to the required high photon flux of soft x-ray radiation, up to now, both methods are almost exclusively performed at synchrotron sources. To overcome this limitation there have been successful efforts to employ laboratory-scale sources based on gas discharge or laser-produced plasmas using liquid and solid laser targets. A serious drawback of these sources is the almost unavoidable generation of debris particles, which can damage optical elements close to the plasma. The operation of liquid jet targets requires also great experimental and technical effort. Thus, there is still the need for further optimization and simplification of lab-scale soft $x$-ray sources to pave the way for their wider dissemination.

Here, the development of a laser-produced plasma source from a pulsed gas jet is presented, enabling the construction of a clean, compact and long-term stable soft $\mathrm{x}$-ray source. As its photon yield is smaller due to the lower particle density within the laser focus, several ways to increase the source brilliance have been examined in detail. In particular, it was investigated how higher gas pressures, shorter laser pulses and smaller emission angles affect the plasma properties. Based on this source a compact transmission $x$-ray microscope operating in the "water window" $(\lambda=2.3-4.4 \mathrm{~nm})$ was built, characterized and applied for imaging of various test and biological objects. The resolving power of the microscope is about 50 to $60 \mathrm{~nm}$. In addition, an advanced laboratoryscale NEXAFS spectrometer has been established, allowing for the reliable analysis of different absorption edges at photon energies between 250 to $1250 \mathrm{eV}$. So far, NEXAFS experiments with the table-top spectrometer have been performed in a high vacuum system, excluding a number of interesting samples from spectroscopic investigations. To extend the application possibilities of the spectrometer, the setup was modified to measure samples also under atmospheric conditions using two silicon nitride membranes as vacuum windows. Finally, different schemes have been suggested to merge the soft x-ray microscope and the NEXAFS spectrometer to a combined laboratory-scale spectromicroscope, which shall enable spatially resolved elemental mapping in the future. It has already been shown that a test object can be imaged at two wavelengths below and above the titanium L-edge. 

1 Einleitung 1

2 Quellen weicher Röntgenstrahlung 5

2.I Synchrotron und Freie-Elektronen-Laser . . . . . . . . . 7

2.1.1 Synchrotron ...................... 7

2.1.2 Freie-Elektronen-Laser . . . . . . . . . . . 9 9

2.2 Hohe Harmonische . . . . . . . . . . . . . . . . . II

2.2.1 Drei-Schritt-Modell . . . . . . . . . . . . . 12

2.2.2 Eigenschaften hoher Harmonischer . . . . . . . . . 15

2.3 Laserinduzierte Plasmen und Entladungsquellen ...... 15

2.3.1 Grundlagen der Plasmaphysik . . . . . . . . . . . 16

2.3.2 Targetkonzepte................. 21

2.3.3 Aufbau der Laserplasmaquelle . . . . . . . . . . 23

2.3.4 Emissionseigenschaften und Brillanzsteigerung . . . . 24

2.3.5 Optimierung der Plasmaquelle . . . . . . . . 35

3 Mikroskopie mit weicher Röntgenstrahlung 39

3.1 Grundlagen .................... . . 40

3.1.1 Kontrastmechanismen und Auflösungsvermögen . . . 40

3.1.2 Optische Elemente . . . . . . . . . . . . . . 45

3.1 .3 Kohärenz . . . . . . . . . . . . . . . . . 54

3.1.4 Konzepte der Röntgenmikroskopie . . . . . . . . . . . . . 56

3.1.5 Dosis und Strahlenschäden . . . . . . . . . . . . 61

3.2 Stand der Forschung . . . . . . . . . . . . . 62

3.3 Laborröntgenmikroskop . . . . . . . . . . . . . . . . 65

3.3.1 Mikroskopaufbau ................ . . . 65

3.3 .2 Testobjekte . . . . . . . . . . . 72

3.3.3 Biologische und geologische Proben . . . . . . . . 77

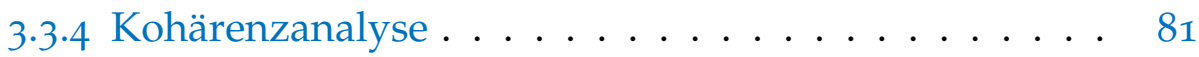

3.3.5 Weiterentwicklung des Röntgenmikroskops . . . . . 85

4 NEXAFS-Spektroskopie $\quad 89$

4.I Feinstruktur der Absorptionskante . . . . . . . . . . . . . . . 89

4.2 Messung von NEXAFS-Spektren . . . . . . . . . . . . . . . . . 92

4.3 Stand der Forschung . . . . . . . . . . . . . . . 93

4.4 Laborröntgenspektrometer . . . . . . . . . . . . . . . . . 95

4.4.1 Gitterjustage und -kalibration . . . . . . . . . . . . . 97

4.4 .2 Auflösungsvermögen . . . . . . . . . . . . . 98 
4.5 Untersuchte Materialsysteme . . . . . . . . . . . . . . 104 4.5.1 Epoxidharz Agar-100 . . . . . . . . . . . . . . 105

4.5.2 Eisenminerale und Silikate . . . . . . . . . . 108

4.6 Ausblenden der 0. Ordnung . . . . . . . . . . . 117

4.7 Proben unter Atmosphärendruck . . . . . . . . . . 120

4.8 Weiterentwicklung des NEXAFS-Spektrometers . . . . . . 124

5 Röntgen-Spektromikroskopie $\quad 127$

5.1 Synchrotronbasierte Spektromikroskopie . . . . . . . . . 127

5.2 Laboraufbau . . . . . . . . . . . . . . . . 128

5.2.1 Zonenplattenmonochromator ........... . . 129

5.2.2 Gittermonochromator . . . . . . . . . . . . . . 129

5.2.3 Zwei-Farbmikroskop ............. 130

6 Zusammenfassung $\quad 135$

A Anhang 139

A.I Instrumentierung . . . . . . . . . . . . . . . . . . 139

A.2 PrismSPECT Simulationsparameter . . . . . . . . . 140

A.3 Fitparameter .................. 140

$\begin{array}{lr}\text { B Naturkonstanten } & 144\end{array}$

$\begin{array}{ll}\text { C Abkürzungen } & 145\end{array}$

$\begin{array}{lr}\text { Abbildungsverzeichnis } & \mathbf{1 4 6}\end{array}$

$\begin{array}{lr}\text { Tabellenverzeichnis } & \mathbf{1 5 0}\end{array}$

$\begin{array}{ll}\text { Literaturverzeichnis } & \mathbf{1 5 3}\end{array}$

$\begin{array}{lr}\text { Eigene Publikationen } & \mathbf{1 8 8}\end{array}$

$\begin{array}{lr}\text { Danksagung } & 191\end{array}$ 


\section{EIN LEIT UNG}

Mit der Erfindung des Lichtmikroskops Ende des 16. Jahrhunderts [1] öffnete sich die Tür zu einer Welt, die dem menschlichen Auge bis dahin verborgen blieb. Um immer tiefer in diese Welt vordringen zu können, wurden mit der Zeit leistungsfähigere Mikroskope gebaut und neue Mikroskopiemethoden entwickelt, die zu unzähligen Entdeckungen in den Naturwissenschaften, insbesondere der Medizin geführt haben. Mikroskope sind daher für zahlreiche wissenschaftliche und industrielle Anwendungen mittlerweile unverzichtbar, aber auch zur Klärung aktueller Forschungsfragen von besonderer Bedeutung. So stellt z. B. die Abbildung lebender Zellen bis heute eine große Herausforderung dar: Die Auflösung klassischer Lichtmikroskope ist auf etwa $200 \mathrm{~nm}$ begrenzt, da aufgrund des Beugungslimits nur Strukturen aufgelöst werden können, die größer sind als die halbe Wellenlänge des verwendeten Lichts [2]. Mit speziellen Fluoreszenzmikroskopen (u. a. STED [3], PALM [4] und STORM [5]) lässt sich die Auflösungsgrenze klassischer Lichtmikroskope überwinden, jedoch müssen dazu die Zellbestandteile gezielt mit geeigneten Farbstoffen markiert werden. Auch mit Elektronenmikroskopen wird eine hinreichend hohe Auflösung von bis zu 0,1 nm erreicht. Dennoch sind sie zur Abbildung lebender Zellen nicht geeignet, weil die Proben vakuumstabil sein müssen. Außerdem ist die Probendicke für Messungen in Transmission auf wenige 10 bis $100 \mathrm{~nm}$ beschränkt. Mit Rasterelektronenmikroskopen lassen sich wiederum nur oberflächennahe Bereiche untersuchen.

Beide Beschränkungen - das begrenzte Auflösungsvermögen klassischer Lichtmikroskope und die zum Teil aufwendige Probenpräparation für die hochauflösende Fluoreszenzmikroskopie - entfallen, wenn zur Bildgebung Röntgenstrahlung verwendet wird. Dabei wird zwischen weicher Röntgenstrahlung mit Wellenlängen von etwa 5 bis $0,3 \mathrm{~nm}$ und harter Röntgenstrahlung mit Wellenlängen bis $1 \mathrm{pm}$ unterschieden [6]. Aufgrund der kürzeren Wellenlängen ist die räumliche Auflösung entsprechend besser als mit sichtbarem Licht. Zudem lassen sich biologische Zellen bis zu einer Dicke von mehreren Mikrometern direkt ohne Anfärbung abbilden. Im Spektralbereich harter Röntgenstrahlung werden dafür hauptsächlich die Röntgenfluoreszenzmikroskopie [7], aber auch weitere Methoden wie die kohärente diffraktive Bildgebung [8] oder In-Line-Holografie [9, 10] verwendet. Kohärente Röntgenbildgebung wird zunehmend auch für weiche Röntgenstrahlung eingesetzt [11] und ergänzt damit klassische Röntgenmikroskope, die bereits zur 
Untersuchung biologischer Proben im Spektralbereich des „Wasserfensters" $(\lambda=2,3-4,4 \mathrm{~nm})$ etabliert sind [12, 13]. Aufgrund des natürlichen Absorptionskontrastes zwischen Kohlenstoff und Wasser werden chemische und strukturelle Informationen über das biologische Objekt gewonnen. Da weiche Röntgenstrahlung mehrere Mikrometer in eine Zelle eindringen kann, ist die Röntgenmikroskopie darüber hinaus nicht auf ultradünne Schnitte angewiesen. Daher ergeben sich zahlreiche Anwendungen für die 3D-Bildgebung intakter biologischer Zellen [14-16].

Weiche Röntgenstrahlung lässt sich aber nicht nur für bildgebende Verfahren, sondern auch für die Absorptionsspektroskopie anwenden, weil in diesem Spektralbereich Absorptionskanten verschiedener Elemente z. B. von Kohlenstoff, Sauerstoff und Eisen liegen. Daraus resultiert ein elementspezifischer Kontrast, der genutzt werden kann, um die chemische Zusammensetzung einer Probe zu bestimmen. Von besonderem Interesse ist die Untersuchung der Feinstruktur einer Absorptionskante im Rahmen der NEXAFS-Spektroskopie (engl. near-edge x-ray absorption fine structure). Die Merkmale der Spektren liefern den "chemischen Fingerabdruck" der Probe und ermöglichen damit Aussagen über die chemische Struktur z. B. über Bindungsarten, Oxidationsstufen und Koordination. Wird die hohe chemische Sensitivität der NEXAFSSpektroskopie mit der hochauflösenden Bildgebung kombiniert, ergeben sich für die (Spektro-)Mikroskopie mit weicher Röntgenstrahlung zahlreiche Anwendungen nicht nur in der Biologie, sondern u. a. auch in der Halbleitertechnik [17] sowie den Material- [18], Geo- [19] und Umweltwissenschaften [20].

Für alle Anwendungen sind allerdings Röntgenquellen hoher Brillanz notwendig, sodass Röntgenmikroskope und -spektrometer fast ausschließlich an Synchrotronen betrieben werden. Da die Nachfrage nach Messzeit an Synchrotronstrahlquellen aber immer weiter zunimmt, sind diese massiv überbucht und röntgenmikroskopische Anwendungen auf einen kleinen Nutzerkreis beschränkt. Für eine weitere Verbreitung der Methoden wurden daher zunehmend Gasentladungs- und laserinduzierte Plasmaquellen zur Erzeugung weicher Röntgenstrahlung im Labormaßstab entwickelt. Das erste Laborröntgenmikroskop, mit dem reproduzierbar wasserfreie Objekte mit hoher Auflösung $(<100 \mathrm{~nm})$ und hohem Signal-Rausch-Verhältnis abgebildet werden konnten, wurde im Jahr 1999 von Hertz et al. [21] präsentiert. Es basiert auf einem laserinduzierten Plasma, wobei als Lasertarget flüssiges Ethanol in Tröpfchenform verwendet wurde. Wenige Jahre später konnte ein kompaktes Röntgenmikroskop auch mit einer Gasentladungsquelle realisiert werden [22]. Ein Nachteil der Gasentladungs- und Plasmaquellen mit festem oder flüssigem Lasertarget ist die unvermeidbare Erzeugung von Debristeilchen, die umliegende Optiken beschädigen [23]. Die Verwendung von Flüssig- und Kryotargets ist zudem aufgrund der benötigten 
Pumpleistungen und der Kühlung mit Flüssigstickstoff mit hohem experimentellem und technischem Aufwand verbunden, der eine kompaktere Bauweise der Systeme verhindert. Diese Einschränkungen entfallen beim Einsatz eines gasförmigen Lasertargets, das den Aufbau sauberer, kompakter und stabiler Quellen für weiche Röntgenstrahlung ermöglicht [24-26]. Der einzige Nachteil ist ihre geringe Brillanz aufgrund der niedrigeren Teilchendichte des Gastargets im Laserfokus und der damit verbundenen relativ großen Ausdehnung im Vergleich zu Plasmen mit Flüssig- oder Festkörpertargets.

Im Rahmen dieser Arbeit wurden daher neue Methoden zur Brillanzsteigerung des laserinduzierten Plasmas untersucht und umgesetzt. Diese werden ausführlich in Kapitel 2 beschrieben. Unter anderem wurde eine neue Düse getestet, mit der sich der Gasdruck um den Faktor 5 erhöhen lässt. Außerdem wurde der Einfluss der Laserpulslänge auf die Emissionseigenschaften des Plasmas und die Winkelabhängigkeit der vom Plasma emittierten Röntgenstrahlung untersucht. Die Fortschritte bei der Entwicklung der Plasmaquelle ermöglichten den Bau eines kompakten Röntgenmikroskops. Neben der umfassenden Charakterisierung des Labormikroskops konnten erste Abbildungen verschiedener Test- und biologischer Objekte mit monochromatischer Strahlung bei $\lambda=2,88 \mathrm{~nm} \mathrm{im} \mathrm{"Wasserfenster"} \mathrm{realisiert} \mathrm{werden,} \mathrm{wobei} \mathrm{das} \mathrm{räumli-}$ che Auflösungsvermögen des Mikroskops im Bereich von 50 bis $60 \mathrm{~nm}$ liegt. Der Aufbau des Mikroskops und die röntgenmikroskopischen Aufnahmen werden ausführlich in Kapitel 3 diskutiert.

Parallel dazu wurde ein bereits vorhandenes NEXAFS-Laborspektrometer weiterentwickelt, mit dem Proben bisher hauptsächlich bei Photonenenergien zwischen 280 und $500 \mathrm{eV}$, insbesondere an der KohlenstoffK-Kante vermessen wurden [27-29]. Das Ziel der Spektrometerentwicklung war deshalb, die zuverlässige Messbarkeit auch von Absorptionskanten bei Energien oberhalb von 500 eV wie der K-Kante des Sauerstoffs oder der L-Kanten von Mangan und Eisen zu erreichen. Zudem mussten die Proben für NEXAFS-Messungen bislang unter Vakuum gehalten werden. Um die damit verbundenen Einschränkungen (wie etwa bei der Analyse von flüssigen oder ausgasenden Proben) zu vermeiden, wurde eine spezielle Kammer gebaut, in der die Proben unter Atmosphärendruck in einer Heliumumgebung untersucht werden können. Die Vorgehensweise und entsprechenden Resultate werden detailliert in Kapitel 4 erläutert.

Synchrotronbasierte Röntgenmikroskope werden heutzutage fast ausschließlich in Kombination mit spektroskopischen Methoden eingesetzt, um die Elementverteilung innerhalb der Probe mit hoher räumlicher Auflösung zu erfassen. In Kapitel 5 werden zwei verschiedene Konzepte für kompakte Spektromikroskope auf Basis laserinduzierter Plasmaquellen mit gasförmigem Target vorgestellt, die zukünftig zu einer weite- 
ren Verbreitung dieser Anwendung über die Nutzergemeinschaft von Synchrotronstrahlquellen hinaus führen könnten. In diesem Zusammenhang wurde mit dem Röntgenmikroskop zudem ein erstes Testobjekt ober- und unterhalb der Titan-L-Kante aufgenommen.

Die drei wesentlichen Ziele dieser Arbeit bestanden demnach in der Brillanzerhöhung der laserinduzierten Plasmaquelle mit gasförmigem Target, dem Bau eines Labormikroskops für weiche Röntgenstrahlung im „Wasserfenster" und der Weiterentwicklung des NEXAFS-Spektrometers. Um ein tieferes Verständnis dieser Arbeitsschwerpunkte zu erlangen, werden zu Beginn der vier Kapitel jeweils zunächst die theoretischen Grundlagen und der aktuelle Stand der Forschung erläutert. Daraufhin werden die einzelnen Maßnahmen beschrieben, mit denen diese Ziele erreicht wurden, und die Ergebnisse der Arbeit diskutiert. Jedes der Kapitel schließt mit einem kurzen Ausblick auf zukünftige Experimente bzw. mit Vorschlägen zur weiteren Optimierung der kompakten Laboraufbauten ab. Zum Abschluss der Arbeit werden in Kapitel 6 die wichtigsten Ergebnisse noch einmal knapp zusammengefasst. 
Seit ihrer Entdeckung durch Wilhelm Conrad Röntgen im Jahr 1895 [30] wird Röntgenstrahlung in zahlreichen wissenschaftlichen und industriellen Anwendungen (siehe Kap. I) genutzt. Dafür stehen einerseits Synchrotrone und Freie-Elektronen-Laser und andererseits Laborquellen wie Röntgenlaser, Röntgenröhren, Entladungs- und Plasmaquellen sowie hohe Harmonische zur Verfügung. Bei allen Quellen wird Röntgenstrahlung infolge starker Beschleunigung von Elektronen und / oder aufgrund hochenergetischer Übergänge in der Elektronenhülle von Atomen oder Molekülen erzeugt. Trotzdem unterscheiden sich die Quellen teils wesentlich in ihren Emissionseigenschaften wie emittierter Wellenlänge, Kohärenz, Divergenz und Pulslänge. Um die Röntgenquellen dennoch klassifizieren und vergleichen zu können, wird u. a. deren Brillanz $\mathrm{Br}$ angegeben. Diese ist definiert als Anzahl der pro Zeiteinheit $\tau$ (in $\mathrm{s}$ ) und Quellfläche $A_{Q}\left(\right.$ in $\mathrm{mm}^{2}$ ) in einen Raumwinkel $\Omega$ (in $\operatorname{mrad}^{2}$ ) emittierten Photonen $N_{P h}$ der Wellenlänge $\lambda$ im Spektralbereich $\Delta \lambda$ [31]:

$$
\mathrm{Br}=\frac{\mathrm{N}_{\mathrm{Ph}}}{\tau \cdot \mathrm{A}_{\mathrm{Q}} \cdot \Omega \cdot \Delta \lambda / \lambda}
$$

wobei die spektrale Breite $\Delta \lambda$ typischerweise $0,1 \%$ der zentralen Wellenlänge $\lambda$ beträgt. Unterschieden wird zudem zwischen Spitzen- und mittlerer Brillanz, je nachdem ob $\tau$ die Pulsdauer der emittierten Strahlung oder die inverse Repetitionsrate angibt. Abbildung 2.I zeigt einen Vergleich von Spitzen- und mittlerer Brillanz der wichtigsten Röntgenquellen. Im Spektralbereich weicher Röntgenstrahlung lässt sich derzeit die höchste Brillanz mit Freie-Elektronen-Lasern (z. B. X-FEL, LCLS) und Synchrotronen der 3. Generation (z. B. PETRA III, ESRF, NSLS) erreichen [33], deren Funktionsweise in Abschnitt 2.I erläutert wird.

Bei den Laborquellen erzielen Röntgenlaser die höchste Brillanz. Allerdings ist die Umsetzung nach dem „klassischen“ Laserprinzip analog zum sichtbaren Spektralbereich aus mehreren Gründen problematisch. Zum einen stehen geeignete Resonatorspiegel mit hinreichend hoher Reflektivität für weiche Röntgenstrahlung nur eingeschränkt zur Verfügung. Als Lasermedium werden daher stark angeregte Plasmen hoher Dichte genutzt, in denen der Röntgenpuls durch den Prozess der "Amplified Spontaneous Emission“ (ASE) im Einzeldurchlauf (,single pass“) verstärkt wird [35]. Zum anderen skaliert die benötigte Pumpleistung $^{1}$ zur Anregung des Mediums mit der Laserwellenlänge $\lambda$ aufgrund

1 Verglichen mit einem Laser der Wellenlänge $500 \mathrm{~nm}$ ist die benötigte Pumpleistung eines Röntgenlasers für $\lambda=5 \mathrm{~nm}$ um einen Faktor $10^{12}$ höher [36]. 

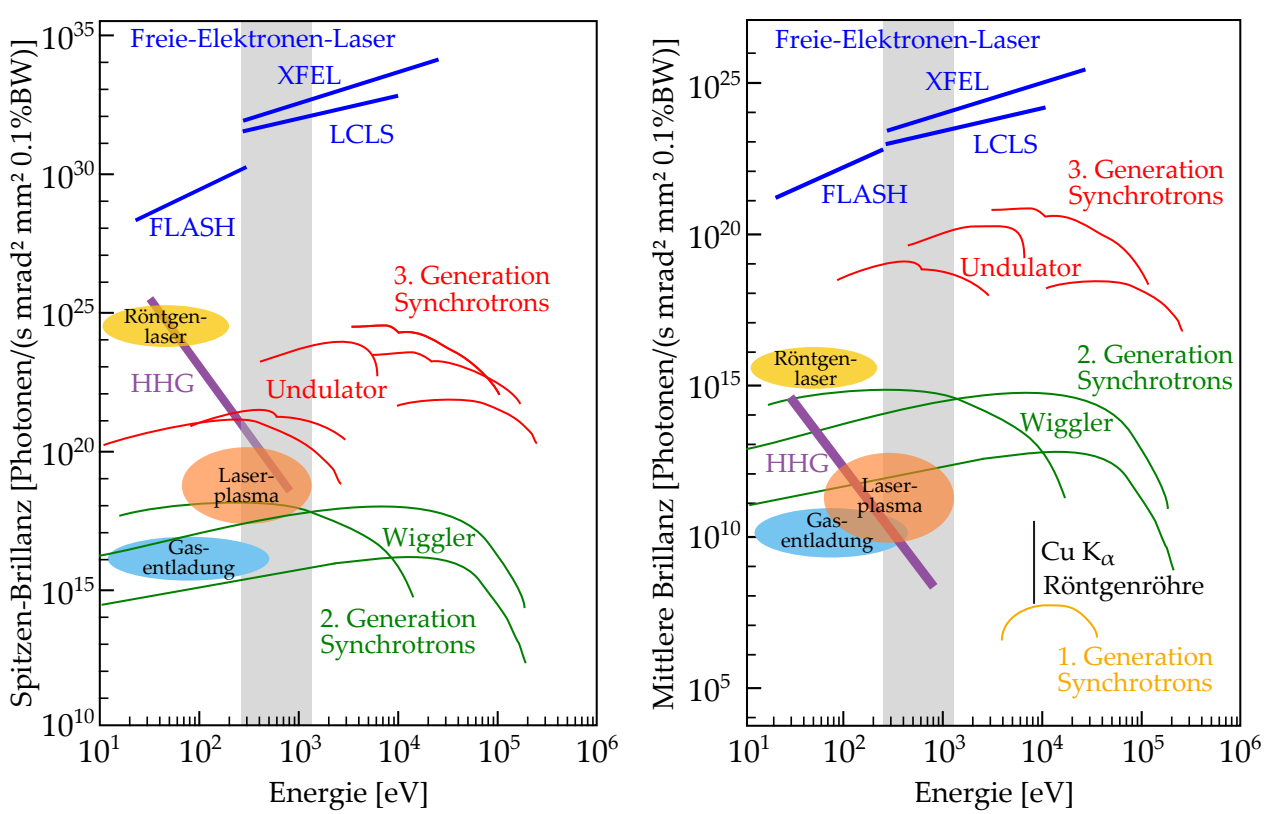

Abbildung 2.1: Spitzen- (links) und mittlere Brillanz (rechts) ausgewählter Röntgenquellen (nach [26, 32-34]). Der für diese Arbeit relevante Energiebereich von 250 bis $1250 \mathrm{eV}$ ist grau hinterlegt.

von Verlusten durch spontane Emission mit $1 / \lambda^{4}$ [37]. Bisher ist der Betrieb kompakter Röntgenlaser deshalb auf Photonenenergien kleiner als 250 eV begrenzt [38].

Röntgenröhren hingegen werden üblicherweise nur zur Erzeugung von Strahlung im Energiebereich einiger Kiloelektronvolt eingesetzt. Dazu werden an einer Kathode freie Elektronen erzeugt und in einem elektrischen Feld zu einer Anode beschleunigt. Beim Auftreffen auf die Anode wechselwirken die Elektronen mit den Anodenatomen und werden dabei stark abgebremst. Dabei wird breitbandige Bremsstrahlung emittiert, die je nach Anodenmaterial von charakteristischen Linien überlagert ist. Der Wirkungsgrad einer Röntgenröhre ist proportional zur Kernladungszahl Z des Anodenmaterials und umgekehrt proportional zur Wellenlängen $\lambda_{\max }$, bei der die Intensität der Bremsstrahlung ihr Maximum erreicht [39]. Die ohnehin schon geringe Effizienz von Röntgenröhren ist demnach für weiche Röntgenstrahlung um etwa drei Größenordnungen kleiner als für harte Röntgenstrahlung bei $10 \mathrm{keV}$, da neben der größeren Wellenlänge gemäß Moseley'schem Gesetz als Anodenmaterial nur Elemente geringer Ordnungszahl wie Lithium $(Z=3)$, Beryllium $(Z=4)$ oder Bor $(Z=5)$ verwendet werden können. Für bildgebende Verfahren oder Spektroskopie mit weicher Röntgenstrahlung werden daher keine Röntgenröhren, sondern hohe Harmonische sowie Plasma- und Entladungsquellen eingesetzt. Diese drei Quellen werden in den Abschnitten 2.2 und 2.3 genauer erklärt, wobei der Schwerpunkt auf der in dieser Arbeit verwendeten laserinduzierten Plasmaquelle liegt. 


\subsection{SYNCHROTRON UND FREIE-ELEKTRONEN-LASER}

Im Jahr 1944 beschrieben Iwanenko und Pomeranchuk [40] eine Art von Strahlung, welche infolge der starken Beschleunigung von Elektronen auftritt, die sich mit relativistischer Geschwindigkeit im Magnetfeld eines Speicherrings bewegen. Drei Jahre danach wurde diese Strahlung an einem Synchrotron bei General Electric (USA) erstmals nachgewiesen [41] und entsprechend Synchrotronstrahlung genannt. Charakteristische Eigenschaften wie der kleine Abstrahlwinkel und die hohen Intensitäten, die andere Quellen insbesondere im Röntgenbereich weit übertreffen, ermöglichen vielfältige Anwendungen von Synchrotronstrahlung u.a. in den Materialwissenschaften, der Kristallografie, Chemie, Biophysik und Medizin [42]. Zunächst als „Nebenprodukt" der Ringbeschleuniger (Synchrotrone der 1. Generation) genutzt, werden seit den 1970ern Anlagen eigens zur Erzeugung von Synchrotronstrahlung gebaut und im Hinblick auf ihre Brillanz stetig weiterentwickelt. Die neuesten Quellen sind Freie-Elektronen-Laser. Die spezifischen Unterschiede im Aufbau der Synchrotronstrahlungsquellen und deren Auswirkungen auf die Strahlungseigenschaften werden im Folgenden diskutiert.

\subsubsection{SYNCHROTRON}

In einem Ringbeschleuniger werden geladene Teilchen (z. B. Elektronen) durch Anlegen eines Magnetfeldes auf annähernd kreisförmige Bahnen gezwungen. Aufgrund der relativistischen Geschwindigkeiten, der kontinuierlichen Richtungsänderung und damit verbundenen Beschleunigung der Elektronen emittieren diese tangential zu ihrer Bewegungsrichtung Synchrotronstrahlung (siehe Abb. 2.2). Weil die Strahlung in Beschleunigungsrichtung der Elektronen abgestrahlt wird, ist sie entlang der horizontalen Achse linear polarisiert. Die Pulslänge liegt im SubNanosekundenbereich. Das Spektrum ist breitbandig und reicht von Infrarot- bis zu harter Röntgenstrahlung [42].

Um die Synchrotronstrahlung eines Ringbeschleunigers zu charakterisieren, wird häufig die kritische Photonenenergie $E_{c}$ angegeben, die das Spektrum in Bereiche gleicher integraler Strahlungsleistung teilt. Sie lässt sich aus der endlichen Pulsdauer der Synchrotronstrahlung ableiten und ist von der Elektronenenergie $E_{e}$ und der magnetischen Flussdichte B abhängig [6]:

$$
\mathrm{E}_{\mathrm{c}}[\mathrm{keV}]=0,665 \cdot \mathrm{E}_{e}^{2}[\mathrm{GeV}] \cdot \mathrm{B}[\mathrm{T}] \text {. }
$$

Zur Erzeugung weicher Röntgenstrahlung $\left(E_{c}=1 \mathrm{keV}\right)$ müssen die Elektronen in einem Magnetfeld der Stärke $B=1 \mathrm{~T}$ demnach auf eine Energie von $\mathrm{E}_{e}=1,25 \mathrm{GeV}$ beschleunigt werden. 


\section{Ringbeschleuniger}

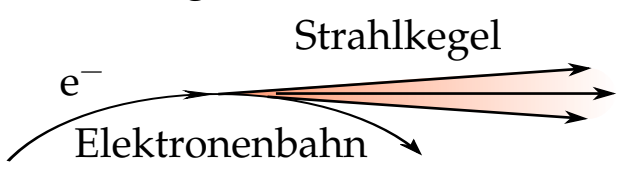

Wiggler

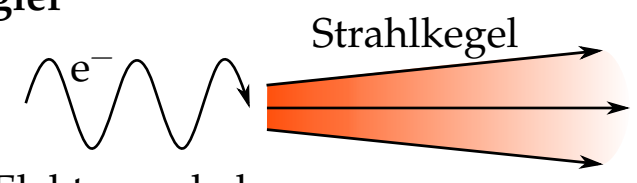

Elektronenbahn

\section{Undulator}

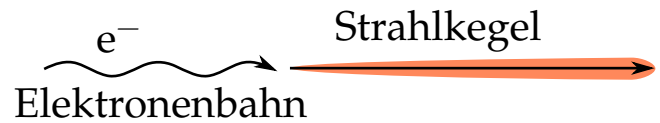

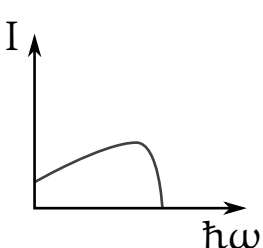
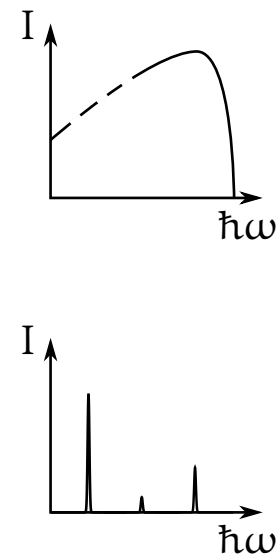

Abbildung 2.2: Synchrotronstrahlung und zugehöriges Spektrum eines Ringbeschleunigers (oben), Wigglers (Mitte) und Undulators (unten; nach [6, 31])

Es gibt allerdings effizientere Möglichkeiten Synchrotronstrahlung zu erzeugen, als Elektronen in einem Ringbeschleuniger auf einer einfachen Kreisbahn zu bewegen. In modernen Synchrotronen (2. und 3. Generation) ist die Kreisbahn durch gerade Abschnitte unterbrochen, in denen die Elektronen durch eine Abfolge von Magneten zu oszillierenden Bewegungen gezwungen werden. Diese sogenannten „insertion devices“ lassen sich in zwei Kategorien unterteilen: Wiggler und Undulatoren (siehe Abb. 2.2). Beide Komponenten bestehen aus einer Reihe von $2 \mathrm{~N}$ Dipolmagneten (typischerweise $N=100$ ), deren Magnetfeldrichtung alterniert. Dadurch werden die Elektronen auf eine meist sinusförmige Bahn gezwungen, sukzessive abgelenkt und damit beschleunigt. Im Wiggler werden die Elektronen dabei sehr viel stärker ausgelenkt als im Undulator, wodurch die erzeugten Strahlungskeulen einen großen Winkel zur Achse des Wigglers einschließen. Da die emittierte Strahlung demnach nicht interferieren kann, ist das Spektrum ähnlich breitbandig wie das eines Ringbeschleunigers. Die Intensität der Wigglerstrahlung ist jedoch um den Faktor 2N höher [31].

Im Undulator wird gezielt eine geringere magnetische Flussdichte als im Wiggler verwendet, sodass die Elektronen weniger stark ausgelenkt werden. Daher interferieren die Strahlungskeulen bei einer Wellenlänge (oder den Harmonischen) konstruktiv miteinander und die Undulatorstrahlung ist quasi-monochromatisch mit Bandbreite $1 / \mathrm{N}$ und partiell kohärent [42]. Obwohl aufgrund des schwächeren Magnetfelds die Beschleunigung der Elektronen und damit die erreichbare Photonenener- 
gie kleiner ist, weist Undulatorstrahlung aufgrund der geringeren Divergenz eine höhere Brillanz auf als Wigglerstrahlung. Zudem skaliert die Intensität der emittierten Strahlung mit $N^{2}$ und nicht mit $N$ wie für den Wiggler. In Synchrotronen der 3. Generation werden deshalb ausschließlich Undulatoren verwendet.

\subsubsection{FREIE-ELEKTRONEN-LASER}

Undulatoren sind hervorragend zur Erzeugung brillanter Synchrotronstrahlung geeignet. Dennoch können sie hinsichtlich ihrer Brillanz übertroffen werden. Denn obwohl sich die Strahlung eines Elektrons kohärent überlagert, ist die Strahlung verschiedener Elektronen zueinander inkohärent. Das liegt daran, dass die Elektronen den Undulator als Elektronengas ohne räumliche Ordnung passieren. Allerdings wechselwirkt die Synchrotronstrahlung mit den Elektronen. In einem FreieElektronen-Laser wird dies gezielt genutzt, um eine kohärente Emission von Synchrotronstrahlung zu erreichen. Das Prinzip des Freie-Elektronen-Lasers wurde 1971 von John Madey [43] vorgestellt. In seiner Arbeitsgruppe gelang sechs Jahre später die erste experimentelle Umsetzung bei einer Wellenlänge von $\lambda=3,4 \mu \mathrm{m}$ unter Verwendung eines optischen Resonators [44]. In den letzten Jahrzehnten wurden Freie-ElektronenLaser zur Erzeugung immer kurzwelliger Strahlung bis in den weichen und harten Röntgenbereich [33, 45, 46] entwickelt.

Die Strahlung überholt die Elektronen nach einer Undulatorperiode. Je nach Phase nehmen die Elektronen entweder Energie von der Strahlung auf und werden infolge der Lorentzkraft beschleunigt oder sie geben Energie ab und werden gebremst. Schnellere Elektronen schließen so zu langsameren auf: Es bildet sich eine Dichtemodulation, deren Periode gerade der Wellenlänge der Strahlung entspricht. Dieser Effekt wird als "Microbunching“ bezeichnet [47]. Die Elektronen eines Paketes sind in Phase und emittieren kohärente Strahlung, die innerhalb des Undulators immer weiter verstärkt wird. Die Intensität skaliert demnach nicht nur quadratisch mit den Undulatorperioden $\left(\propto N^{2}\right)$, sondern ist auch proportional zum Quadrat der Anzahl der in einem Paket enthaltenen Elektronen $\left(\propto n_{\#}^{2}\right)$. Zudem zeichnet sich die Strahlung durch eine geringe Divergenz, kurze Pulsdauern im Bereich weniger Femtosekunden und einen hohen Kohärenzgrad aus. Freie-Elektronen-Laser erreichen daher eine viel höhere Brillanz als z. B. Synchrotrone der 3. Generation.

Da im Röntgenbereich keine Spiegel mit hinreichender Reflektivität zum Bau optischer Resonatoren zur Verfügung stehen, können die Elektronen den Undulator nur einmal durchlaufen. Die Undulatoren in FreieElektronen-Lasern sind daher mehrere zehn bis 100 Meter lang, damit sich die Elektronendichtemodulation vollständig ausbildet. Die Strahlungsleistung $P$ wächst dabei zunächst linear mit der im Undulator zu- 
rückgelegten Wegstrecke $z$ an, bis die Feldstärke hoch genug ist, um die Elektronenverteilung entsprechend zu modulieren. Infolge dieser Wechselwirkung nimmt die Leistung sodann exponentiell zu [46]:

$$
\mathrm{P} \propto \mathrm{e}^{z / l_{9}}
$$

Die Länge $l_{g}($,gain length“) charakterisiert dabei die Verstärkungsrate und beträgt üblicherweise einige Meter. Nachdem die Elektronen eine Wegstrecke von etwa $22 l_{g}$ zurückgelegt haben [33], steigt die Leistung nicht weiter: Die Elektronen geben genauso viel kinetische Energie an das elektromagnetische Feld ab, wie sie zur Aufrechterhaltung der Resonanzbedingung aus dem Feld aufnehmen [48]. Der Verlauf der Strahlungsleistung innerhalb des Undulators ist in Abbildung 2.3 dargestellt.

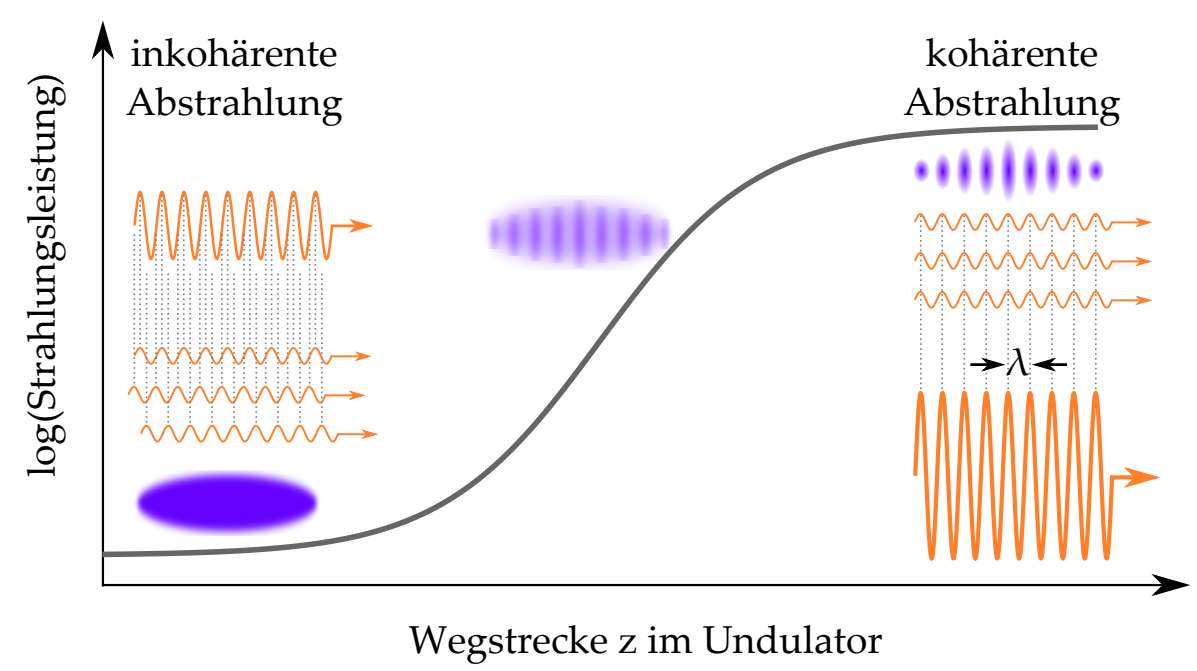

Abbildung 2.3: Strahlungsleistung als Funktion der im Undulator zurückgelegten Wegstrecke $z$ nach dem SASE-Prinzip und Modulation der Elektronendichte (nach $[46,47])$.

Der Verstärkungsprozess beginnt aufgrund spontaner Fluktuationen im Elektronenstrahl und wird daher selbstverstärkte spontane Emission (SASE - „Self-Amplified Spontaneous Emission“) genannt [49]. Einzelne Pulse unterscheiden sich in Intensität, Zeitstruktur und spektralen Eigenschaften [50]. Zudem ist das Spektrum relativ breitbandig, wodurch die zeitliche Kohärenz verringert ist [51]. Um Puls-zu-Puls Schwankungen zu minimieren, wird der Elektronenstrahl bereits vor Eintritt in den Undulator z. B. mit einem Laser moduliert [52]. Alternativ kann eine Selbstmodulation auftreten, wenn die Undulatorstrahlung mit einem Monochromator gefiltert wird. Dadurch verringert sich die relative Bandbreite um einen Faktor 40 bis 50 [53].

Freie-Elekronen-Laser erreichen Gesamtlängen von einigen Kilometern (z. B. European XFEL: 3,4 km [54]). Daher wird der Bau kompakter 
Freie-Elektronen-Laser im Labormaßstab angestrebt [55-57]. Bereits verfügbare Laborquellen zur Erzeugung weicher Röntgenstrahlung wie hohe Harmonische, laserinduzierte Plasmen und Entladungsquellen werden in den folgenden Abschnitten vorgestellt.

\subsection{HOHE HARMONISCHE}

Die nichtlineare Wechselwirkung eines intensiven Laserpulses der Frequenz $\omega_{0}$ mit einem Konversionsmedium führt zur Erzeugung hoher Harmonischer mit Frequenz $m \cdot \omega_{0}$ [32], wobei $m$ eine ganze positive Zahl ist. Nachdem bereits 1961 die 2. Harmonische eines Rubin-Lasers bei $\lambda=347 \mathrm{~nm}$ in einem Quartzkristall beobachtet wurde [58], konnten McPherson et al. [59] und Ferray et al. [60] erst Ende der 1980er hohe Harmonische bis zur 17. bzw. 33. Ordnung in Neon, Argon und Xenon erzeugen. Bis heute wurden hohe Harmonische im Bereich weicher Röntgenstrahlung bis zu Photonenenergien von 1,3 keV demonstriert [61]. Zudem werden hohe Harmonische zur Erzeugung von ultrakurzen Pulsen im Attosekundenbereich [62-64] eingesetzt. Abbildung 2.4 zeigt ein charakteristisches Spektrum hoher Harmonischer, die in Edelgasen erzeugt werden.

Ordnung $\mathrm{m}$

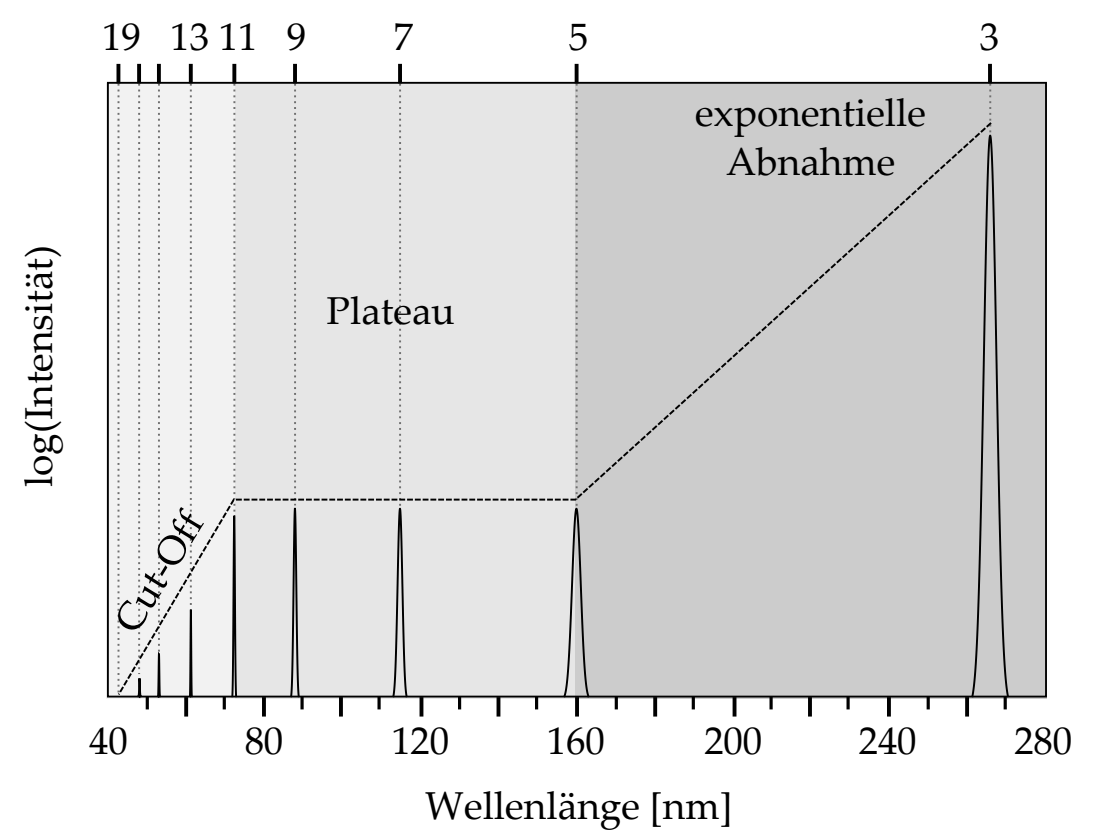

Abbildung 2.4: Exemplarisches Spektrum bei der Erzeugung hoher Harmonischer (Fundamentale $800 \mathrm{~nm}$ ) in Edelgasen (nach [32, 65]).

Die Entstehung hoher Harmonischer lässt sich durch das 1993 von Corkum [66] und Kulander et al. [67] entwickelte, semi-klassische „DreiSchritt-Modell“ - Ionisation, Propagation, Rekombination - beschreiben. 


\section{SCHRITT 1: IONISATION}

Im ersten Schritt zur Erzeugung hoher Harmonischer wird ein freies Elektron generiert. Der zugrunde liegende Mechanismus hängt dabei vom elektrischen Feld $E(t)=E_{0} \cos (\omega t)$ des Laserpulses $a b$, weil dadurch zusätzlich zum Coulomb-Term des Atoms $V_{C}$ der Potenzialterm $V_{\mathrm{L}}=e \mathrm{E}(\mathrm{t}) \mathbf{r}$ auftritt. Die Elektronen befinden sich demnach in einem effektiven Potenzial [32]:

$$
V_{\text {eff }}(\mathbf{r}, t)=-\frac{e^{2}}{4 \pi \epsilon_{0} r}+e E(t) r
$$

Dabei bezeichnet $\epsilon_{0}$ die elektrische Feldkonstante und $r$ den Abstand eines Elektrons vom Atom. Das Ionisationspotential $I_{P}$ ist mit dem ponderomotorischen Potential (mittlere kinetische Energie)

$$
\mathrm{U}_{\mathrm{P}}[\mathrm{eV}]=\frac{\mathrm{e}^{2} \mathrm{E}_{0}^{2}}{4 \mathrm{~m}_{\mathrm{e}} \mathrm{\omega}^{2}}=0,97 \cdot 10^{-13} \cdot \mathrm{I}\left[\mathrm{W} \mathrm{cm}^{-2}\right] \cdot \lambda^{2}\left[\mu \mathrm{m}^{2}\right]
$$

über den Keldysh-Parameter $\gamma_{\mathrm{K}}=\sqrt{\mathrm{I}_{\mathrm{P}} /\left(2 \mathrm{U}_{\mathrm{P}}\right)}$ verknüpft [68]. Für Laserintensitäten von $\mathrm{I} \approx 10^{15} \mathrm{~W} \mathrm{~cm}^{-2}$ beträgt das ponderomotorische Potenzial bei einer Wellenlänge von $\lambda=800 \mathrm{~nm}$ etwa $60 \mathrm{eV}$.

Abhängig von Intensität I und Frequenz $\omega$ bzw. Wellenlänge $\lambda$ des elektrischen Feldes wird zwischen drei Ionisationsmechanismen unterschieden:

- Bleibt das Coulomb-Potenzial unverändert, ist Multi-Photonenabsorption die einzige Möglichkeit, um ein freies Elektron zu erzeugen (siehe Abb. 2.5a).

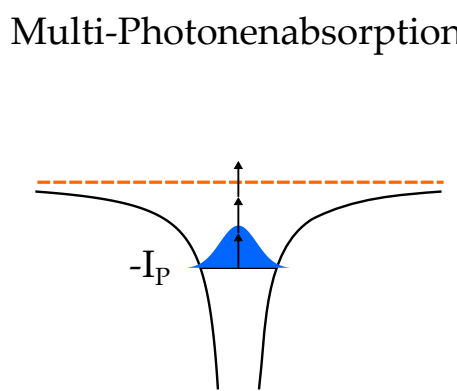

(a)
Tunnelionisation

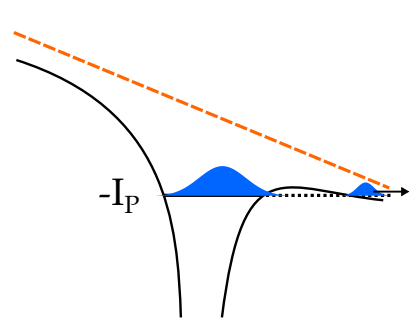

(b)
Feldionisation

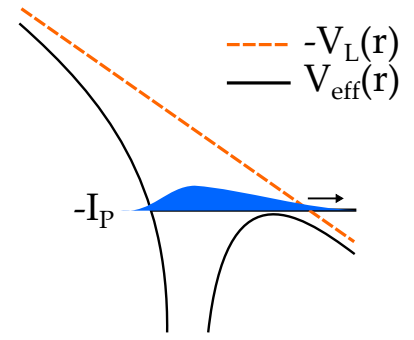

(c)

Abbildung 2.5: Mechanismen zur Erzeugung freier Elektronen (nach [32]). IP bezeichnet das Ionisationspotenzial, $V_{\mathrm{L}}$ ist der Potenzialterm aufgrund des Laserpulses und $V_{\text {eff }}$ das effektiv auf das Elektron (blau) wirkende Potenzial. 
- Bei höherer Feldstärke wird das Coulomb-Potenzial so weit abgesenkt, dass die gebundenen Elektronen mit einer endlichen Wahrscheinlichkeit über die verbleibende Barriere aus dem Atom hinaustunneln können (siehe Abb. 2.5b). Die Periode $2 \pi / \omega$ des elektrischen Feldes muss dazu verglichen mit der Tunnelzeit hinreichend lang sein, damit das Elektron das Atom verlassen kann, bevor das Feld seine Richtung ändert, und das Potenzial wieder anhebt.

- Liegt die Ionisationsenergie oberhalb des Coulomb-Potenzials (Feldionisation, siehe Abb. 2.5c), können die Elektronen das Atom „klassisch" verlassen.

\section{SCHRITT 2: PROPAGATION}

Nach der Ionisation wird das Elektron näherungsweise als freies geladenes Teilchen betrachtet, dessen Bewegung allein durch das elektrische Feld $E(t)=E_{0} \cos (\omega t)$ des Laserpulses bestimmt ist. Auf das Elektron wirkt die $\operatorname{Kraft} F(t)=-e E(t)$, sodass es auf die Geschwindigkeit

$$
v(t)=\int_{0}^{t}-\frac{e}{m_{e}} E\left(t^{\prime}\right) d t^{\prime}+v_{0}
$$

beschleunigt wird. Die Ionisation kann zu einer beliebigen Phase $\varphi$ stattfinden. Unter der Annahme, dass das Elektron zur Zeit $t=0$ am Ort $x_{0}=0$ mit der Geschwindigkeit $v_{0}=0$ an das Elektron gebunden ist, lauten die Bewegungsgleichungen für ein Elektron demnach [32]

$$
\begin{aligned}
& v(t)=-\frac{e E_{0}}{m_{e} \omega}[\sin (\omega t+\varphi)-\sin (\varphi)] \quad \text { und } \\
& x(t)=\frac{e E_{0}}{m_{e} \omega^{2}}[\cos (\omega t+\varphi)-\cos (\varphi)+\sin (\varphi) \cdot \omega t] .
\end{aligned}
$$

Die Amplitude der oszillatorischen Bewegung wird als ponderomotorischer Radius $a_{p}=e E_{0} /\left(m_{e} \omega^{2}\right)$ bezeichnet und liegt für Laserintensitäten von $\mathrm{I} \approx 10^{15} \mathrm{~W} \mathrm{~cm}^{-2}$ und einer Wellenlänge von $\lambda=800 \mathrm{~nm} \mathrm{im}$ Bereich weniger Nanometer. Abbildung 2.6a zeigt die Trajektorien $x(t)$ der Elektronen für verschiedene Phasenverschiebungen $\varphi$. Für kleine Phasen $\varphi \approx 0$ bleibt das Elektron nah bei seinem Ausgangsion. Je größer allerdings die Phase wird, desto schneller entfernt sich das Elektron vom Ursprung $x=0$. Dadurch kehren deutlich weniger Elektronen zu ihrem Ion zurück und rekombinieren.

\section{SCHRITT 3: REKOMBINATION}

Rekombiniert ein Elektron mit seinem Ausgangsion, wird meist ein Photon emittiert. Die Energie des Photons ist dabei die Summe aus der Io- 


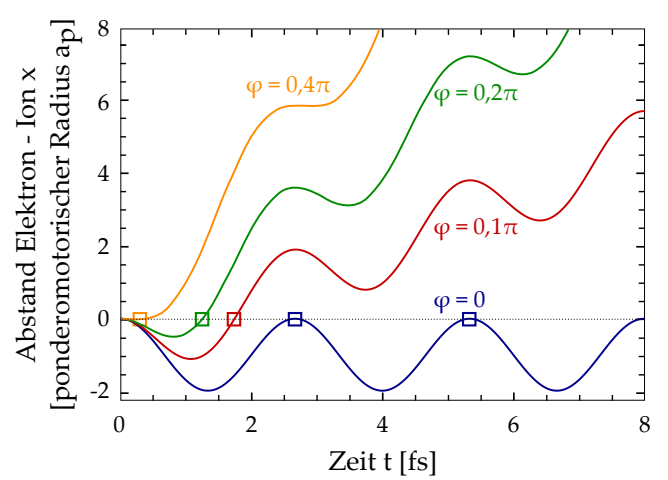

(a) Trajektorien

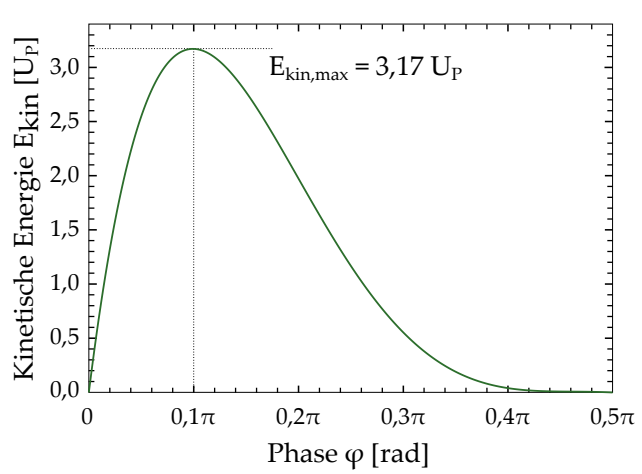

(b) kinetische Energie

Abbildung 2.6: (a) Trajektorien der Elektronen, die zum Zeitpunkt $t=0$ mit einer Phasenverschiebung $\varphi$ im elektrischen Feld des Laserpulses $(\lambda=800 \mathrm{~nm})$ beschleunigt werden (nach [32]). Orte möglicher Rekombination der Elektronen mit ihren Ausgangsionen sind durch Quadrate gekennzeichnet. (b) Kinetische Energie der Elektronen zum Zeitpunkt $t_{0}$ der Rekombination mit dem Ausgangsion als Funktion der Phase $\varphi$.

nisationsenergie $I_{P}$ und der kinetischen Energie des Elektrons $E_{k i n}$ das im elektrischen Feld des Laserpulses beschleunigt wurde:

$$
\hbar \omega=I_{P}+E_{k i n}
$$

Geschwindigkeit und kinetische Energie der Elektronen zum Zeitpunkt $t_{0}$ der Rekombination entsprechen der Steigung der Trajektorien am Ort $x=0$ (siehe Abb. 2.6a) und sind von der Phase $\varphi$ abhängig. Der Zeitpunkt $t_{0}(\varphi)$ kann daher durch numerisches Lösen der Gleichung $x\left(t_{0}\right)=0$ für verschiedene Phasen bestimmt und daraufhin die kinetische Energie gemäß $E_{\text {kin }}=m_{e} / 2 \cdot v\left(t_{0}\right)^{2}$ berechnet werden (siehe Abb. 2.6b). Für eine Phase von $\varphi=0,1 \pi$ ist die kinetische Energie mit einem Wert von $\mathrm{E}_{\mathrm{kin}}^{\max } \approx 3,17 \mathrm{U}_{\mathrm{P}}$ maximal. Die Energie der emittierten Photonen kann demnach nicht höher sein als

$$
\hbar \omega_{c}=I_{P}+3,17 U_{P}
$$

und hohe Harmonische können folglich nur bis zu einer Grenzfrequenz $\omega_{c}$ erzeugt werden. Dieser Zusammenhang wurde von Kulander et al. [69] bereits vor der Veröffentlichung des „Drei-Schritt-Modells“ empirisch hergeleitet.

Zur Erzeugung kurzwelliger Strahlung mit Photonenenergien von 250 bis $1000 \mathrm{eV}$ sind bei einer Wellenlänge von $\lambda=800 \mathrm{~nm}$ Intensitäten von bis $\mathrm{zu} \mathrm{I}=10^{16} \mathrm{~W} \mathrm{~cm}^{-2}$ notwendig. Daher werden für hohe Harmonische hauptsächlich Femtosekundenlaser mit Wellenlängen im Infrarotbereich eingesetzt (siehe z. B. [64]). Der dominierende Mechanismus zur Ionisation des Konversionsmediums ist dabei der Tunneleffekt. 


\subsubsection{EIGENSCHAFTEN HOHER HARMONISCHER}

Die Erzeugung hoher Harmonischer hängt von der Phase $\varphi$ des elektrischen Feldes ab. Die Strahlung ist dementsprechend kohärent zur Fundamentalen des Laserpulses. Darüber hinaus ist die Emission der Photonen auf einen Bruchteil der halben Periodendauer $T_{0} / 2$ des Laserpulses begrenzt, sodass hohe Harmonische eine sehr kurze Pulslänge im Sub-Femto- bis Attosekundenbereich aufweisen [63]. Zudem wiederholt sich der Entstehungsprozess in Konversionsmedien mit Inversionssymmetrie zweimal pro Laserzyklus. Dies führt im Frequenzraum zu einem Abstand der diskreten Frequenzen von $\Delta \omega=2 \pi /\left(T_{0} / 2\right)=2 \omega_{0}$. Das Spektrum besteht daher aus ungeraden Vielfachen der Frequenz $\omega_{0}$ (siehe Abb. 2.4). In Medien gebrochener Symmetrie wiederholt sich die Erzeugung hoher Harmonischer hingegen nach einer vollen Periode $\mathrm{T}_{0}$. Im Spektrum treten deshalb gerade und ungerade Harmonische auf [32]. Kontinuierliche Strahlung kann unter Verwendung sogenannter „few-cycle"-Laserpulse erzeugt werden [70, 71], indem die Rekombination der Elektronen mit den Ionen so gezielt gesteuert wird, dass nur zu einem Zeitpunkt des Laserpulses Photonen emittiert werden.

\subsection{LASERINDUZIERTE PLASMEN UND ENTLADUNGSQUELLEN}

Eine nach außen elektrisch neutrale makroskopische Ansammlung geladener Teilchen wird allgemein als Plasma bezeichnet [72]. Um ein Plasma zu generieren, wird z. B. ein Laserstrahl mit hinreichend hoher Intensität $\left(10^{12}\right.$ bis $\left.10^{15} \mathrm{~W} / \mathrm{cm}^{2}\right)$ auf ein Target (siehe Abschnitt 2.3.2) fokussiert. Die Dichte solcher laserinduzierter Plasmen ist fast so hoch wie die eines Festkörpers, und die Temperatur liegt im Bereich mehrerer Millionen Kelvin. Daher eignen sich diese sogenannten heißen, dichten Plasmen hervorragend als Quellen weicher Röntgenstrahlung.

Eine Alternative zur laserbasierten Erzeugung eines Plasmas ist die elektrische Gasentladung. Obwohl die Umwandlung elektrischer Energie in Strahlung sehr effizient ist, erreicht das Plasma zunächst keine hinreichend hohe Temperatur zur Emission weicher Röntgenstrahlung. Um das Plasmas entsprechend aufzuheizen, wird in Gasentladungsquellen vorwiegend der Pinch-Effekt genutzt [73]: Die gerichtete Bewegung der geladenen Teilchen (elektrischer Strom) führt zur Entstehung eines Magnetfeldes. Infolge der resultierenden Lorentzkraft bewegen sich die Teilchen aufeinander zu und das Plasma wird verdichtet.

Gasentladungsquellen unterscheiden sich stark hinsichtlich ihrer Entladungsgeometrie. Zur Erzeugung weicher Röntgenstrahlung werden die „pseudo spark-like“ Elektrodenanordnung [74], die „micro-plasmapulsed“ Entladung [75] und der "Z-Pinch“ [76] am häufigsten genutzt. Problematisch ist in allen Fällen die Debrisproduktion durch die Erosion 
der Elektroden aufgrund der hohen Spitzenstromstärken und der thermischen Last durch den geringen Abstand zum Plasma. Um die Lebensdauer der Gasentladungsquelle zu erhöhen und Debris zu reduzieren, wurde z. B. der elektrodenlose "Z-Pinch" entwickelt [77].

Aufgrund des Pinch-Effektes sind die Plasmen typischerweise zylinderförmig. Bei einer Länge von wenigen Millimetern beträgt ihr Durchmesser einige $100 \mu \mathrm{m}$. Die Pulslänge der emittierten - inkohärenten Röntgenstrahlung liegt im Bereich von Nanosekunden [74]. Je nach Elektrodengeometrie erreichen Gasentladungsquellen eine Repetitionsrate von bis $\mathrm{zu} 1 \mathrm{kHz}$ [22].

Die emittierte Strahlung von Plasmen unterscheidet sich von der hoher Harmonischer z. B. hinsichtlich Pulslänge und Kohärenz. Um diese Unterschiede zu verstehen, werden im nächsten Abschnitt zunächst die grundlegenden Plasmaprozesse erklärt. Daran anschließend wird die experimentelle Umsetzung zur Erzeugung laserinduzierter Plasmen beschrieben, und es werden die Emissionseigenschaften der Plasmaquelle im Hinblick auf das Erreichen einer möglichst hohen Brillanz diskutiert.

\subsubsection{GRUNDLAGEN DER PLASMAPHYSIK}

Das physikalische Verhalten und die Eigenschaften eines Plasmas werden durch kurz- und langreichweitige Wechselwirkungen der freien negativen und positiven elektrischen Ladungen untereinander und mit externen elektromagnetischen Feldern z. B. Laserstrahlung charakterisiert.

Kurzreichweitige Prozesse umfassen $u$. a. inelastische Stöße zwischen den Elektronen und Atomen. Dabei wird das Plasma aufgeheizt und die Atome werden ionisiert, wobei der Anregungsgrad wesentlich von der Elektronentemperatur und den Bindungsenergien der Atome abhängt. Zudem entstehen weitere freie Elektronen, die wiederum mit Atomen zusammenstoßen können. Die daraus resultierende Elektronenlawine liefert zahlreichen Ionen in hoch angeregten Zuständen. Findet ein Übergang aus dem angeregten Zustand $n$ in einen energetisch tiefer liegenden Zustand $m$ statt, wird schmalbandige Linienstrahlung der Energie $\Delta E_{n m}=h v_{n m}=E_{n}-E_{m}$ emittiert, die mit dem verallgemeinerten Moseley'schen Gesetz berechnet werden kann [78]:

$$
h v_{n m}=\frac{h c_{0} R_{\infty}}{1+m_{e} / m_{k}}(Z-S)^{2}\left(\frac{1}{m^{2}}-\frac{1}{n^{2}}\right)
$$

Dabei ist $R_{\infty}$ die Rydberg-Konstante, $m_{K}$ die Kernmasse, $c_{0}$ die Lichtgeschwindigkeit und Z die Ordnungszahl des Elements. Die Konstante S beschreibt die Abschirmung der Kernladung durch die Elektronen.

Zugleich entsteht breitbandige Brems- und Rekombinationsstrahlung, wenn die Elektronen im Coulombfeld der Atome und Ionen beschleunigt werden bzw. mit den Ionen rekombinieren. Weil die Abstände zu 
den Ionen, die Geschwindigkeiten und damit kinetischen Energien der Elektronen verschieden sind, ist die emittierte Strahlung kontinuierlich. Ein charakteristisches Emissionsspektrum ist in Abbildung 2.7 gezeigt.

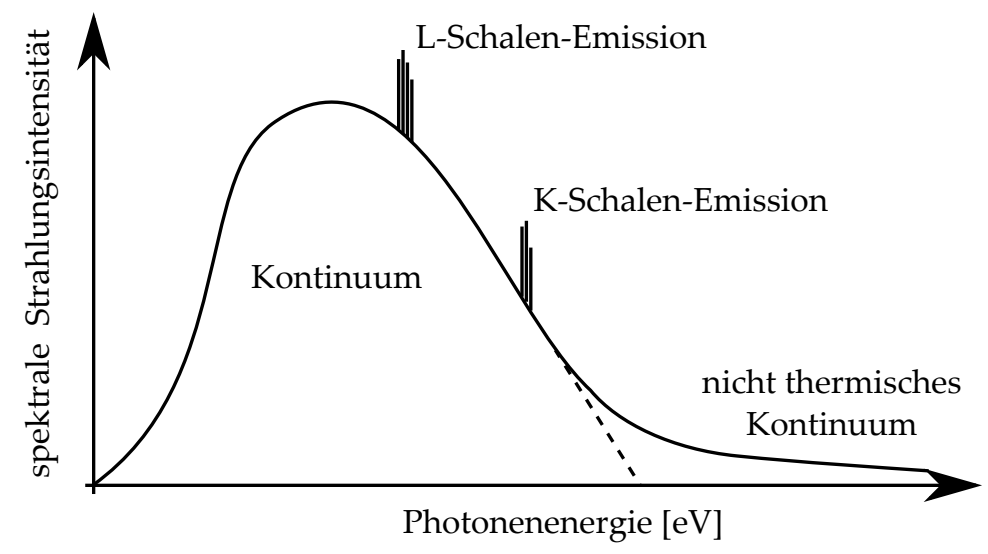

Abbildung 2.7: Emissionsspektrum eines heißen, dichten Plasmas (nach [6]). Der kontinuierliche Anteil des Spektrums resultiert aus Brems- und Rekombinationsstrahlung. Die schmalbandigen Linien entstehen aufgrund diskreter Übergänge in unterschiedlich stark angeregten Ionen. Der nicht thermische Ausläufer zu hohen Energien ist auf „heiße“ Elektronen zurückzuführen.

Zur Beschreibung der Prozesse in einem Plasma wird in erster Näherung angenommen, dass sich das Plasma im thermischen Gleichgewicht befindet. Den Elektronen und Ionen wird dabei eine gemeinsame Temperatur $T_{e}$ mit dazugehöriger Maxwell'scher Geschwindigkeitsverteilung $f$ zugewiesen [79]:

$$
f(v)=\sqrt{\frac{2}{\pi}} \cdot \frac{v^{2}}{v_{e}^{3}} \cdot e^{-v^{2} /\left(2 v_{e}\right)^{2}} .
$$

Dabei bezeichnet $v_{e}^{2}=k_{\mathrm{B}} T_{e} / m_{e}$ mit Boltzmann-Konstante $k_{\mathrm{B}}$ die mittlere quadratische Geschwindigkeit der Elektronen. Im Rahmen dieser Näherung kann das Plasma als Schwarzer Körper behandelt werden, dessen emittierte Strahlung unabhängig von der Beschaffenheit (Oberfläche, Dichte ...) nur von seiner Temperatur abhängt. Die spektrale Leistungsdichte (in Einheiten der Brillanz)

$$
\mathrm{B}=3,146 \cdot 10^{11} \cdot \frac{(\hbar \omega / \mathrm{eV})^{3}}{e^{\hbar \omega /\left(\mathrm{k}_{\mathrm{B}} \mathrm{T}_{e}\right)}-1} \frac{\text { Photonen } / \mathrm{s}}{\mathrm{mm}^{2} \cdot \mathrm{mrad}^{2} \cdot 0,1 \% \mathrm{BW}}
$$

ergibt sich aus dem Planck'schen Strahlungsgesetz für $\Delta \lambda / \lambda=10^{-3}$ und erreicht für eine Photonenenergie von $\hbar \omega=2,822 \mathrm{k}_{\mathrm{B}} \mathrm{T}_{e}$ ihr Maximum [6]. Die mittlere Elektronentemperatur ${ }^{2}$ laserinduzierter Plasmen mit gasförmigen Targets liegt bei etwa $100 \mathrm{eV}$ [80]. Daraus ergibt sich

2 Ausgedrückt in Einheiten der Energie entspricht eine Elektronentemperatur von $100 \mathrm{eV}$ etwa $1,2 \cdot 10^{6} \mathrm{~K}$. 
bei einer Wellenlänge von $\lambda=4,4 \mathrm{~nm}$ eine maximale Brillanz von etwa $4,5 \cdot 10^{17}$ Photonen $/\left(\mathrm{s} \cdot \mathrm{mm}^{2} \cdot \operatorname{mrad} \cdot 0,1 \% B W\right)$. Die pro Fläche abgestrahlte Leistung I eines idealen Schwarzen Körpers ist gemäß StefanBoltzmann-Gesetz proportional zur vierten Potenz der Elektronentemperatur $\mathrm{T}_{e}[6]$ :

$$
\mathrm{I}=\sigma \mathrm{T}_{e}^{4}=\frac{\pi^{2} \mathrm{k}_{\mathrm{B}}^{4}}{60 \mathrm{c}_{0}^{2} \hbar^{3}} \cdot \mathrm{T}_{e}^{4} \approx 1 \cdot 10^{5} \frac{\mathrm{W}}{\mathrm{cm}^{2} \mathrm{eV}} \cdot\left(\mathrm{k}_{\mathrm{B}} \mathrm{T}_{e}\right)^{4} .
$$

Ein Plasma der Elektronentemperatur von $100 \mathrm{eV}$ strahlt daher mit einer Intensität von ca. $10^{13} \mathrm{~W} / \mathrm{cm}^{2}$. Entsprechend muss der Laserpuls zum Aufheizen des Plasmas mindestens diese Intensität aufweisen, um die Emission weicher Röntgenstrahlung zu gewährleisten.

Tatsächlich befindet sich das Plasma nicht im thermischen Gleichgewicht, und die abgestrahlte Leistung unter Annahme eines Schwarzen Körpers stellt lediglich eine obere Grenze der Emission des Plasmas dar. Infolge nichtlinearer Prozesse wie Resonanzabsorption werden einige Elektronen so stark beschleunigt, dass sie hochenergetische Brems- bzw. Rekombinationsstrahlung emittieren (nicht thermisches Kontinuum siehe Abb. 2.7). Allerdings reicht ein Zwei-Temperatur-Modell meist aus, um die breitbandige Strahlung des Plasmas zu beschreiben [6].

Neben den kurzreichweitigen Prozessen, in denen Energie hauptsächlich durch Stöße zwischen den Teilchen übertragen wird, sind in heißen, dichten Plasmen auch langreichweitige Wechselwirkungen wie PlasmaOszillationen von Bedeutung. Den Übergang von kurz- zu langreichweitigen Phänomenen charakterisiert die Debye-Abschirmlänge

$$
\lambda_{\mathrm{D}}=\sqrt{\frac{\epsilon_{0} \mathrm{k}_{\mathrm{B}} \mathrm{T}_{e}}{\mathrm{e}^{2} \mathrm{n}_{e}}}
$$

in die neben der Elektronentemperatur $T_{e}$ auch die Elektronendichte $n_{e}$ eingeht. Für typische Dichten von $n_{e}=10^{20} \mathrm{e} / \mathrm{cm}^{3}$ [81] und einer Temperatur von $100 \mathrm{eV}$ beträgt die Debye-Abschirmlänge etwa $7 \mathrm{~nm}$. Auf Skalen größer der Debye-Abschirmlänge ist das Plasma quasineutral und es dominieren kollektive Prozesse wie Oszillationen der Elektronenoder Ionendichte. Um das Verhalten der kollektiven Phänomene exakt zu charakterisieren, müssten die Positionen und Geschwindigkeiten aller Teilchen verfolgt werden. Das ist aufgrund der hohen Komplexität analytisch nicht und auch im Rahmen numerischer Simulationen nur für eine stark begrenzte Anzahl von Teilchen möglich. Zur Vereinfachung werden daher die Geschwindigkeiten aller Teilchen gemittelt und das Plasma als Fluid mit den Parametern Dichte, Temperatur und Druck auf Grundlage der Maxwell-Euler Gleichungen beschrieben. Im Folgenden werden die wichtigsten Kenngrößen eines Plasmas z. B. Plasmafrequenz und kritische Dichte diskutiert. Eine detaillierte Herleitung findet sich z. B. in Referenz [6]. 
Die kollektive Elektronenbewegung ist durch die Plasmafrequenz

$$
\omega_{p}=\sqrt{\frac{n_{e} e^{2}}{\epsilon_{0} m_{e}}}
$$

charakterisiert, die der natürlichen Schwingungsfrequenz der Elektronen im Plasma entspricht. Für die Ausbreitung einer transversalen, ebenen elektromagnetischen Welle gilt für den Realteil der Dispersionsrelation $\omega^{2}=\omega_{p}^{2}+k^{2} c_{0}^{2}$ mit der Wellenzahl $k$. Diese unterscheidet sich lediglich durch den Term $\omega_{p}^{2}$ von der Dispersionsrelation elektromagnetischer Wellen im Vakuum, wo sich die Wellen unabhängig von ihrer Frequenz mit der Lichtgeschwindigkeit $c_{0}$ ausbreiten. Im Plasma hingegen wird die Wellenzahl k imaginär, wenn die Frequenz der Strahlung $\omega$ kleiner ist als die Plasmafrequenz $\omega_{p}$. Die elektromagnetische Welle kann sich in diesem sogenannten „überdichten“ Bereich nicht ausbreiten. Aus Gleichung (2.15) folgt für $\omega=\omega_{p}$ die kritische Elektronendichte

$$
n_{c}=\frac{\epsilon_{0} m_{e} \omega^{2}}{e^{2}}=\frac{1,11 \cdot 10^{21} \mathrm{e} / \mathrm{cm}^{3}}{\lambda^{2}[\mu \mathrm{m}]} \text {. }
$$

Für einen Nd:YAG-Laser $(\lambda=1064 \mathrm{~nm})$ beträgt die kritische Elektronendichte somit etwa $10^{21} \mathrm{e} / \mathrm{cm}^{3}$. Nur im "unterdichten“ Bereich des Plasmas $\left(n_{e} \ll n_{c}\right)$ wird die einfallende Strahlung absorbiert, da die elektromagnetische Welle die freien Elektronen im Plasma zu Schwingungen anregt. Einige der Elektronen geben ihre Energie infolge von inelastischen Stößen mit den Ionen ab und heizen so das Plasma auf (siehe Abb. 2.8). Mit zunehmender Dichte nimmt auch die Effizienz des Energietransfers von der elektromagnetischen Welle ins Plasma zu - allerdings nur, bis die kritische Dichte erreicht ist und die elektromagnetische Welle total reflektiert wird. Die Emission der Röntgenstrahlung erfolgt hingegen vorwiegend aus einem schmalen Bereich des Plasmas oberhalb der kritischen Dichte (siehe Abb. 2.8), in den Energie durch geladene Teilchen transportiert wird.

Der Dichtegradient und die damit einhergehende selbstregulierende Heizzone entstehen, weil sich das Plasma mit der Geschwindigkeit

$$
v_{\exp }=\sqrt{\frac{5 \mathrm{Q}_{\mathrm{I}} \mathrm{k}_{\mathrm{B}} \mathrm{T}_{e}}{3 \mathrm{~m}_{\mathrm{I}}}}
$$

ausdehnt. Die Expansionsgeschwindigkeit $v_{\exp }$ hängt demnach neben der Elektronentemperatur $\mathrm{T}_{e}$ auch von der Masse $\mathrm{m}_{\mathrm{I}}$ (Trägheit der Ionen) und der mittleren Ladung $\mathrm{Q}_{\mathrm{I}}$ der Ionen $\mathrm{ab}$. Ein 6fach angeregtes Stickstoffplasma expandiert bei einer Elektronentemperatur von $100 \mathrm{eV}$ mit einer Geschwindigkeit von ca. $80 \mu \mathrm{m} / \mathrm{ns}$. Zum einen muss also die Energiezufuhr auf der gleichen Zeitskala erfolgen, um eine für die Emission von Röntgenstrahlung hinreichend hohe Plasmatemperatur zu erreichen. Zum anderen können keine beliebig kleinen laserinduzierten Plasmen erzeugt werden. 


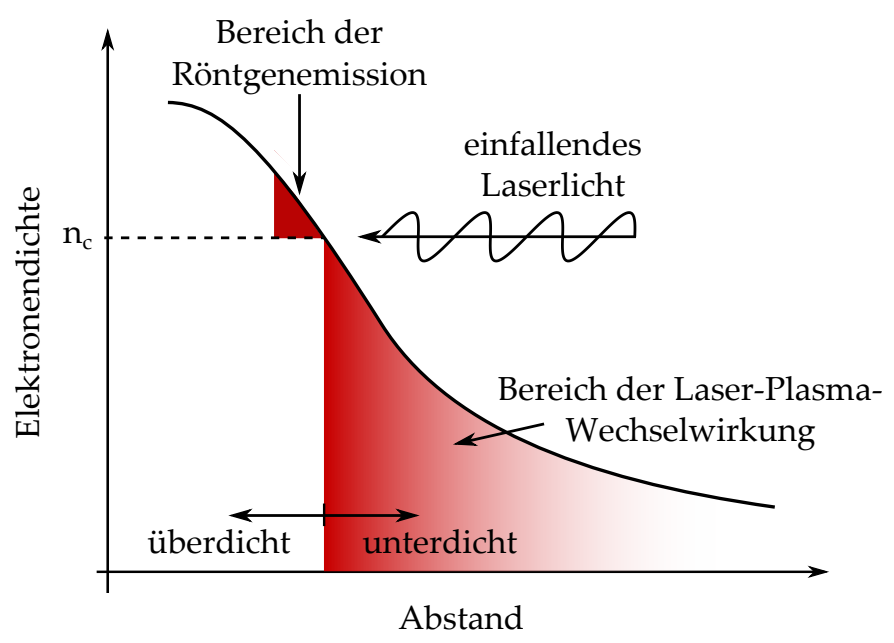

Abbildung 2.8: Eindimensionaler Elektronendichteverlauf eines laserinduzierten Plasmas (nach [6]). Im „unterdichten“ Bereich des Plasmas wird das Laserlicht absorbiert und das Plasma infolge inverser Bremsstrahlung aufgeheizt. Mit Erreichen der kritischen Elektronendichte $n_{c}$ wird die elektromagnetische Welle hingegen total reflektiert. Röntgenstrahlung wird hauptsächlich aus einem schmalen Bereich oberhalb der kritischen Dichte emittiert.

Atomare Prozesse innerhalb eines Plasmas können mithilfe verschiedener Programme simuliert werden. Von besonderem Interesse ist dabei häufig die Abhängigkeit der spektralen Eigenschaften von den charakteristischen Plasmaparametern Elektronentemperatur und -dichte. Um den Einfluss dieser beiden Größen auf das Emissionsspektrum laserinduzierter Plasmen zu untersuchen, wurde im Rahmen dieser Arbeit das Programm PrismSPECT verwendet. Es basiert auf einem „magnetohydrodynamic, collisional-radiative spectral analysis"-Code [82, 83]. Für die Simulation eines Emissionsspektrums, wird zunächst ein passendes Atommodell gewählt. Grundsätzlich werden dabei sowohl die elementspezifischen Energieniveaus als auch die dazugehörigen durch Stöße sowie Strahlung möglichen Übergänge berücksichtigt. Je nach Anwendung beinhaltet das Modell mehr oder weniger Details über die im Plasma vorhandenen chemischen Elemente. Dem Programm PrismSPECT liegt die ATBASE Datenbank zugrunde, die Energieniveaus und Übergangswahrscheinlichkeiten für alle Ionisationsgrade aller Elemente bis zur Ordnungszahl Z = 36 (Krypton) enthält.

Ausgehend vom ausgewählten Modell wird ein System gekoppelter zeitabhängiger Ratengleichungen gelöst, um die Besetzung der einzelnen Niveaus zu berechnen. Daraus ergeben sich die wellenlängen- bzw. frequenzabhängigen Absorptions- und Emissionseigenschaften des Plasmas. Damit können wiederum für verschiedene Elektronentemperaturen und -dichten die spektralen Eigenschaften des Plasmas bestimmt und z. B. mit gemessenen Spektren verglichen werden. 


\subsubsection{TARGETKONZEPTE}

Für laserinduzierte Plasmen werden als Target Festkörper, Flüssigkeiten und Gase verwendet. Die Dichte des Materials bestimmt dabei die Brillanz der Quelle, und es gilt in der Regel: Je höher die Dichte ist, desto mehr Röntgenphotonen werden pro Volumen erzeugt. Laserinduzierte Plasmen mit Festkörpertargets weisen demnach die höchste Brillanz auf. Um eine kontinuierliche Zufuhr des Targetmaterials bei Repetitionsraten von bis $\mathrm{zu} 1 \mathrm{kHz}$ zu gewährleisten, werden $\mathrm{u}$. a. rotierende Zylinder [84, 85] oder schnell umlaufende dünne Folien [86] genutzt. Je nach Anwendung kommen typischerweise Metalle wie Kupfer, Eisen, Aluminium, Zinn, Wolfram, Platin, Gold und Blei $[87,88]$ zum Einsatz. Darüber hinaus werden ausgefrorene Gase [89, 90] oder Flüssigkeiten [91] in ihrer festen Phase - sogenannte Kryotargets - verwendet. Die Plasmen auf der Basis fester Targets zeichnen sich durch eine vergleichsweise geringe Ausdehnung aus, die nur wenige zehn Mikrometer beträgt.

Mit flüssigen Targets, wie Methanol oder Ethanol $[92,93]$ werden ähnliche Plasmagrößen und Brillanzen erreicht. Das Material wird dabei entweder als kontinuierlicher Flüssigkeitsstrahl oder in Form einzelner Tropfen (Droplets) in die Vakuumkammer eingebracht.

Wie in Abschnitt 2.3.I beschrieben, kann der Laserpuls das Plasma nur bis zum Erreichen der kritischen Dichte aufheizen. Aufgrund der intrinsisch hohen Dichte von flüssigen und festen Targetmaterialien wird die Energie des Laserpulses demnach nicht vollständig in das Plasma eingekoppelt. Daher wird die Dichte des Materials häufig zunächst mit einem Vorpuls geringer Intensität reduziert, bevor dann mit dem eigentlichen Hauptpuls das Plasma erzeugt wird [94].

Der weit größere Nachteil bei der Verwendung flüssiger und fester Materialien ist allerdings die unvermeidliche Debrisproduktion, insbesondere von schnellen Teilchen und Ionen mit Energien bis zu $200 \mathrm{keV}$ [95]. Dadurch können empfindliche Optiken beschädigt oder sogar vollständig zerstört werden [96]. Um Debrisschäden zu vermeiden, wurden daher unterschiedliche Konzepte entwickelt: Zum einen werden elektrische [97] und magnetische Felder [98] eingesetzt, um die geladenen Teilchen in Ionenfallen zu lenken. Zum anderen wird zum Abbremsen der Teilchen ein Gas bei niedrigem Hintergrunddruck verwendet [99], oder die Optiken werden gezielt mit Schutzgas umspült [10o]. Trotz dieser Bemühungen bleibt die Lebensdauer der Optiken begrenzt, selbst wenn die genannten Techniken kombiniert eingesetzt werden [101]. Deshalb muss bereits die Erzeugung von Debris verhindert werden. Dazu werden sogenannte „masselimitierte“ Targets eingesetzt, deren Durchmesser so gewählt ist, dass alle Targetatome im Laserfokus in den Plasmazustand überführt werden. Neben den schon erwähnten Droplets kommen hauptsächlich gasförmige Targets zum Einsatz [24, 25]. Das Gas wird 
hierbei über piezoelektrisch oder elektromagnetisch gesteuerte Düsen ins Vakuum expandiert. Um trotz des Gasvordrucks von bis zu 25 bar die Gasmenge in der Vakuumkammer und damit eine mögliche Reabsorption der Röntgenstrahlung zu minimieren, wird die Düse in aller Regel gepulst betrieben. Die Pulslänge liegt typischerweise im Bereich von einigen Mikro- bis Millisekunden. Laserinduzierte Plasmen auf Basis gasförmiger Targets sind nicht nur nahezu debrisfrei, sondern zeichnen sich ebenso durch einen langzeitstabilen Betrieb, eine sehr kompakte Bauweise und einfache Handhabung aus. Darüber hinaus lassen sich die spektralen Eigenschaften der emittierten Strahlung ohne großen Aufwand variieren, indem unterschiedliche Gase verwendet werden (siehe Abs. 2.3.4). Während Gase niedriger Ordnungszahl (z. B. Stickstoff, Sauerstoff) schmalbandige Linienstrahlung emittieren, ist das Spektrum von Gasen hoher Ordnungszahl (z. B. Krypton, Xenon) aufgrund der vielen möglichen elektronischen Übergänge quasi-kontinuierlich [8I].

Die Brillanz der Plasmen ist wegen der niedrigen Dichte des Gastargets und der größeren Ausdehnung des Plasmas allerdings geringer als bei Plasmen, bei denen Flüssigkeiten oder Festkörper als Targetmaterialien verwendet werden. Um die Dichte zu erhöhen, werden daher speziell geformte Düsen [102, 103] bzw. sogenannte Doppelstrahldüsen [104] eingesetzt. Zusätzlich kann der Gasdruck von einigen 10 bar auf 100 bis 200 bar erhöht werden (siehe Abschnitt 2.3.4). Eine andere Möglichkeit zur Verdichtung des Gases besteht darin, die Vakuumkammer mit einem Hintergrundgas geringen Drucks zu füllen. Dadurch wird ein sogenannter „Fass-Stoß“ hervorgerufen, der den Gasstrahl komprimiert. Infolge der höheren Konversionseffizienz von Laserenergie in weiche Röntgenstrahlung lässt sich die Brillanz bis auf das Zehnfache steigern [105]. Neben der Teilchendichte des Targetgases hängen die Emissionseigenschaften der Plasmaquelle wesentlich von der Pulsdauer (bei konstanter Pulsenergie) und damit von der Leistungsdichte des verwendeten Lasers ab (siehe Abschnitt 2.3.4). Werden keine Nano-, sondern Pikosekundenpulse verwendet, verschiebt sich das Emissionsspektrum zu kürzeren Wellenlängen. Zugleich ist die Gesamtzahl der im weichen Röntgenbereich emittierten Photonen fünf- bis zehnmal größer und die Ausdehnung des Plasmas um etwa ein Drittel geringer [8I].

Im Folgenden wird der Aufbau der laserinduzierten Plasmaquelle beschrieben und es werden die Emissionseigenschaften des Plasmas für verschiedene Parameter wie Gasart und -druck sowie Laserpulslänge und -energie diskutiert. Dabei wird insbesondere darauf eingegangen, wie die Brillanz der Plasmaquelle erhöht werden kann. 


\subsubsection{AUFBAU DER LASERPLASMAQUELLE}

Zur Erzeugung kurzwelliger Strahlung im Energiebereich von 250 bis $1250 \mathrm{eV}$ wird eine laserinduzierte Plasmaquelle genutzt (siehe Abb. 2.9), die im Wesentlichen aus einem gepulsten Nano- bzw. Pikosekundenlaser (Parameter siehe Tab. 2.I) und einer piezo-elektrisch gesteuerten Düse nach dem Proch-Trickl-Prinzip [106] besteht. Da die mittlere freie Weg-

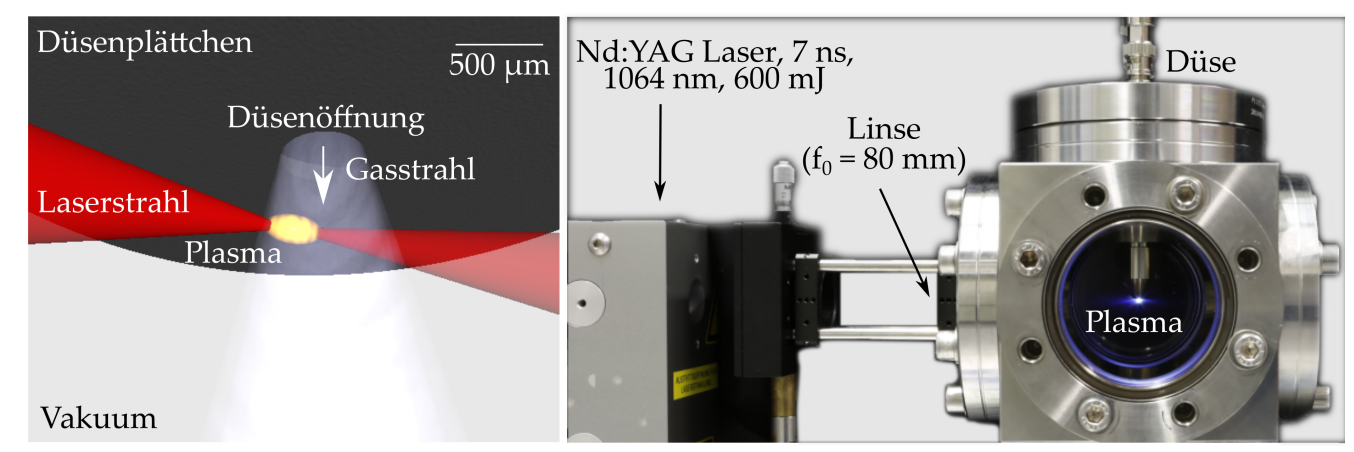

Abbildung 2.9: Schematische Darstellung (links) und Foto des Aufbaus (rechts) zur Erzeugung weicher Röntgenstrahlung

länge weicher Röntgenstrahlung in Luft nur wenige Millimeter beträgt, wurden alle Versuche unter Vakuumbedingungen bei einem Druck kleiner $10^{-2}$ mbar durchgeführt.

Tabelle 2.1: Parameter der eingesetzten Nano- bzw. Pikosekundenlaser

\begin{tabular}{lccc}
\hline & $\begin{array}{c}\text { Innolas } \\
\text { Spitlight 600 }\end{array}$ & $\begin{array}{c}\text { Quantel } \\
\text { YG 980 }\end{array}$ & $\begin{array}{c}\text { Ekspla } \\
\text { SL312P }\end{array}$ \\
\hline Wellenlänge $[\mathrm{nm}]$ & 1064 & 1064 & 1064 \\
Pulslänge $[\mathrm{ns}]$ & 7 & 10 & 0,17 \\
Pulsenergie $[\mathrm{mJ}]$ & 600 & 600 & 500 \\
Repetitionsrate $[\mathrm{Hz}]$ & 5 & 10 & 5 \\
Strahldurchmesser $[\mathrm{mm}]$ & 6 & 8 & 12 \\
Fokusdurchmesser $[\mu \mathrm{m}]$ & 60 & 50 & 40 \\
Leistungsdichte $\left[\mathrm{W} / \mathrm{cm}^{2}\right]$ & $3,0 \cdot 10^{12}$ & $3,1 \cdot 10^{12}$ & $2,3 \cdot 10^{14}$ \\
\hline
\end{tabular}

Das Gas expandiert über ein $1 \mathrm{~mm}$ langes konisch geformtes Düsenplättchen aus Wolfram (Eintrittsdurchmesser $d_{E}=300 \mu m$, Austrittsdurchmesser $d_{A}=550 \mu \mathrm{m}$ ) in den evakuierten Quellwürfel. Die Düse ist dabei lediglich für ca. $900 \mu$ s geöffnet, um den Gasballast und damit eine mögliche Reabsorption der Röntgenstrahlung zu minimieren. Der Gasdruck liegt typischerweise bei 10 bis 20 bar. Der Laserstrahl wird mit einer Linse (Brennweite $\mathrm{f}_{0}=80-120 \mathrm{~mm}$ ) auf den gepulsten Gasstrahl fokussiert. Aufgrund der hohen Leistungsdichte von bis zu $10^{14} \mathrm{~W} / \mathrm{cm}^{2}$ 
im Laserfokus werden im Gas infolge von Multi-Photonenabsorption und Feldionisation freie Elektronen erzeugt (siehe Abschnitt 2.3.I) und es entsteht ein heißes, dichtes Plasma. Obwohl die Teilchendichte an der Austrittsöffnung am höchsten ist, wird das Plasma in einem Abstand von 0,5 bis $1 \mathrm{~mm}$ zur Düse gezündet. Dadurch wird eine Beschädigung des Düsenplättchens durch schnelle Ionen und folglich die Produktion von Debris verhindert.

Um gestreute Laserstrahlung und vom Plasma emittiertes Licht außerhalb des weichen Röntgenbereiches zu blocken, werden je nach Anwendung dünne Metallfolien (Lebow, Dicke 150 bis 200 nm) aus Aluminium, Zirkonium oder Titan eingesetzt.

\subsubsection{EMISSIONSEIGENSCHAFTEN UND BRILLANZSTEIGERUNG}

Einige der in diesem Abschnitt beschriebenen Ideen und gezeigten Abbildungen finden sich so oder so ähnlich in folgender Publikation:

M. Müller, F.-C. Kühl, P. Großmann, P. Vrba und K. Mann, "Emission properties of ns and ps laser-induced soft x-ray sources using pulsed gas jets," Optics Express 21(10), 12831 (2013).

Zur räumlichen Charakterisierung des Plasmas wurde eine Lochkame-

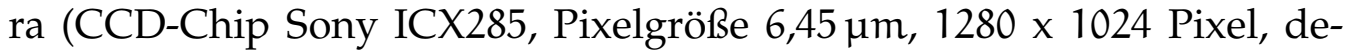
taillierter Aufbau und Funktionsweise siehe Ref. [26]) verwendet. Die Blende (Durchmesser $50 \mu \mathrm{m}$ ) ist mit einer $200 \mathrm{~nm}$ dicken, freitragenden Titanfolie bedeckt, sodass nur Strahlung mit Wellenlängen $<20 \mathrm{~nm}$ zur Kamera gelangen. Der CCD-Chip ist mit Phosphor (ProxiVision, P43, Schichtdicke $5 \mu \mathrm{m}$ ) beschichtet, um die Röntgenstrahlung in sichtbares Licht der Wellenlänge $\lambda=545 \mathrm{~nm}$ zu konvertieren, das dann detektiert wird. Spektral wird das Plasma mit einem Gitterspektrometer (mittlere Gitterkonstante 2400 Linien/mm) analysiert. Das Spektrometer wird ausführlich in Abschnitt 4.4 beschrieben. Eine Übersicht der verschiedenen Möglichkeiten zur Charakterisierung des Plasmas und der dazu verwendeten Messgeräte sowie deren Kenngrößen findet sich zudem in Tabelle A.I im Anhang A.

GASART

Die spektralen Eigenschaften der laserinduzierten Plasmaquelle hängen wesentlich von der Wahl des Targetgases ab. Abbildung 2.10 zeigt die Emissionsspektren von Stickstoff, Sauerstoff, Krypton und Xenon im weichen Röntgenspektralbereich.

Die Spektren der Elemente mit niedriger Ordnungszahl Z (Stickstoff und Sauerstoff) bestehen aus einzelnen, isolierten Linien. Mit zunehmender Ordnungszahl steigt die Anzahl möglicher elektronischer Übergänge allerdings stark an, sodass die Emissionsspektren der Elemente Kryp- 

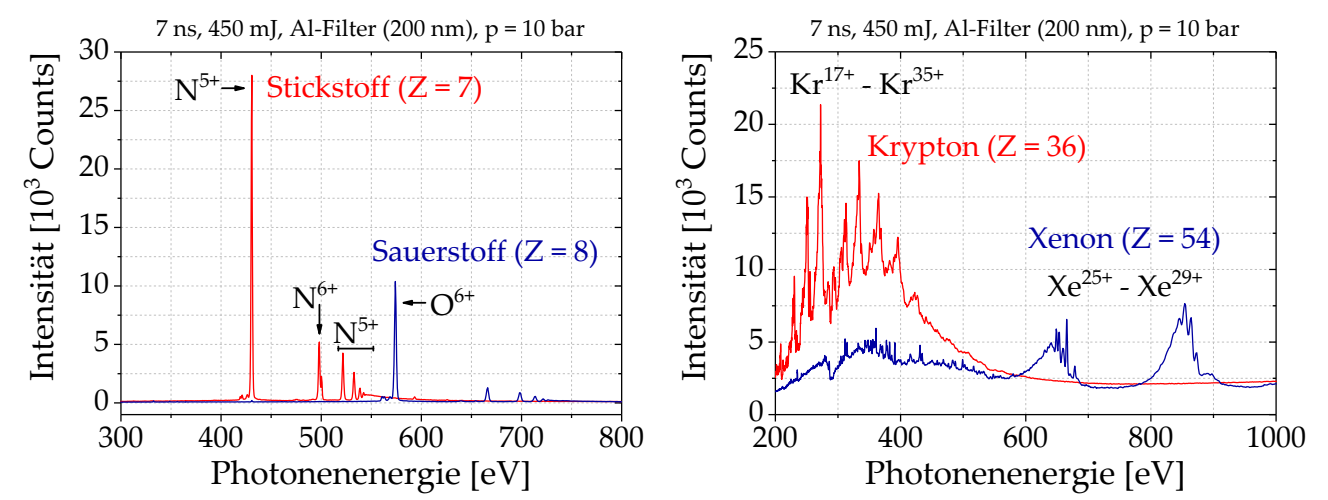

Abbildung 2.10: Emissionsspektren (100 Pulse) verschiedener Gase

ton und Xenon eine Vielzahl von Linien aufweisen. Weil die Übergänge energetisch jedoch so nah nebeneinander liegen, können sie im Spektrometer nicht einzeln aufgelöst werden. Die emittierte Strahlung von Elementen mit hoher Ordnungszahl ist daher quasi-kontinuierlich.

\section{LASERPULSL ÄNGE UND -ENERGIE}

Neben der Wahl des Gases hängen die Emissionseigenschaften des Plasmas auch von der Pulsdauer bzw. bei gleicher Pulsenergie von der Leistungsdichte des verwendeten Lasers ab (siehe Abb. 2.11). Bei der Verwendung des Pikosekundenlasers nimmt die Intensität des Plasmas stark zu, und das Spektrum ist zu höheren Photonenenergien verschoben. Offensichtlich führt die höhere Leistungsdichte des Pikosekundenlasers zu einer stärkeren Ionisation des Gases, was z. B. anhand der Anregung wasserstoffähnlicher Stickstoffionen $\left(\mathrm{N}^{6+}\right)$ deutlich wird. Der Ioni-
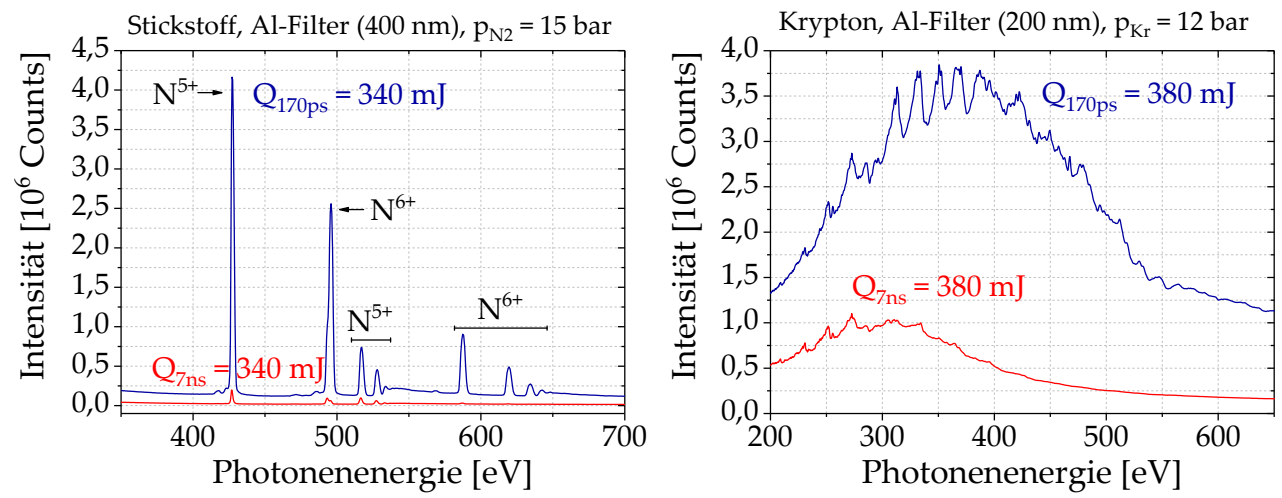

Abbildung 2.11: Emissionsspektren (100 Pulse) von Stickstoff (links) und Krypton (rechts) für verschiedene Pulslängen von $7 \mathrm{~ns}$ bzw. $170 \mathrm{ps}$

sationsgrad hängt dabei wesentlich von der Elektronentemperatur und -dichte des Plasmas ab. Um diese beiden Parameter für die von Nanobzw. Pikosekundenlasern induzierten Plasmen abzuschätzen, wurden Emissionsspektren von Argon mit dem Programm PrismSPECT berech- 

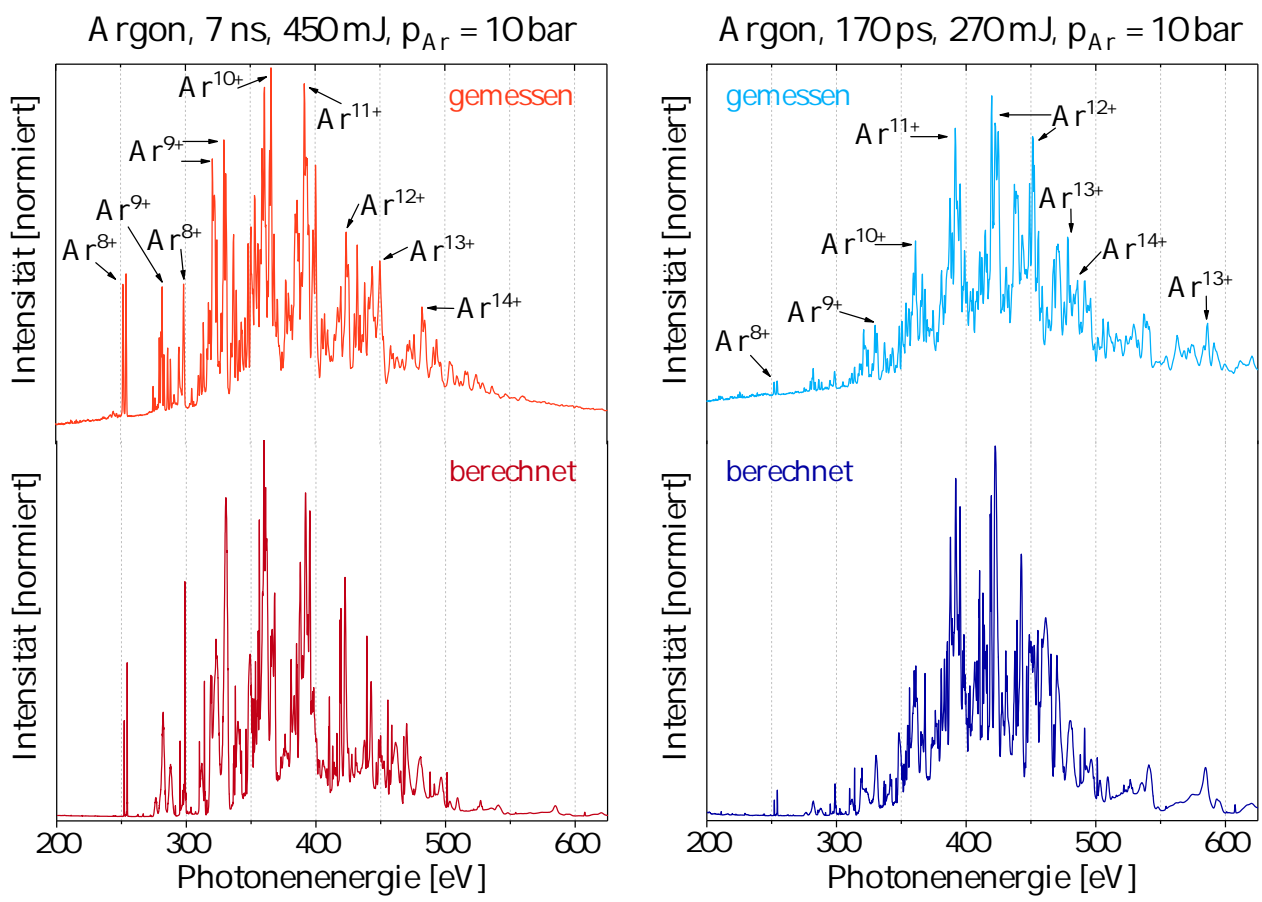

Abbildung 2.12: Vergleich zwischen gemessenen und mit PrismSPECT berechneten Argonspektren (100 Pulse, Al-Filter Dicke $200 \mathrm{~nm}$ ) für $7 \mathrm{~ns}$ (links) und 170 ps (rechts) Pulslänge. Die Parameter der berechneten Spektren sind in Tabelle 2.2, die Programmeinstellungen im Anhang A aufgeführt.

net (siehe Abs. 2.3.I) und mit experimentell bestimmten Spektren verglichen. Die Einstellungen der Simulation sind im Anhang A aufgelistet.

Ein laserinduziertes Plasma befindet sich während der Wechselwirkung mit dem Laserstrahl nicht im thermischen Gleichgewicht und die gemessenen Emissionsspektren repräsentieren eine Mischung von Spektren aus Bereichen unterschiedlicher Elektronentemperatur $T_{e}$ und Elektronendichte $n_{e}$. Daher wurden für verschiedene Parameterkonfigurationen $\left\{T_{e}, n_{e}\right\}$ Spektren berechnet und gewichtet kombiniert, bis eine möglichst gute Übereinstimmung zwischen gemessenen und simulierten Spektren vorlag. In Abbildung 2.12 sind die Emissionsspektren gegenübergestellt: Sowohl für den Nano- als auch den Pikosekundenlaser werden nahezu alle Merkmale der komplexen Spektren bis hin zu kleinen Details reproduziert. Der prozentuale Anteil verschiedener Elektronentemperaturen und -dichten zum Gesamtspektrum und die daraus resultierenden Mittelwerte sind in Tabelle 2.2 zusammengetragen. Unter Verwendung des Pikosekundenlasers erreicht das Plasma eine im Mittel 30 \% höhere Elektronentemperatur. Infolge der stärkeren Ionisation der Targetatome nimmt du Elektronendichte um etwa den Faktor $3 \mathrm{zu}$.

$\mathrm{Zu}$ beachten ist, dass die angegebenen Temperaturen und Dichten nicht eindeutig sind. Es können jedoch keine beliebigen Parameterkombinationen zur Simulation der Spektren verwendet werden. Außerdem 
zeigt die große Übereinstimmung der Emissionsspektren und ein Vergleich der dafür verwendeten Elektronentemperaturen und -dichten mit Daten aus der Literatur [107-109], dass eine hinreichend gute Abschätzung der Plasmaparameter gelungen ist.

Eine ausführlichere Analyse der Emissionseigenschaften wurde für Stickstoff und Krypton durchgeführt, da beide Gase im Bereich weicher Röntgenstrahlung als Target von besonderer Bedeutung sind: Stickstoff wird in Kombination mit einer Titanfolie als quasi-monochromatische Quelle für Linienstrahlung bei $430 \mathrm{eV}$ in der Röntgenmikroskopie genutzt. Krypton hingegen weist ein quasi-kontinuierliches Spektrum auf und ist damit ein ideales Target für spektroskopische Anwendungen. In Abbildung 2.13 sind Emissionsspektren von Stickstoff und Krypton für verschiedene Pulsenergien des Pikosekundenlasers gezeigt. Die Leistungsdichte des Pikosekundenlasers liegt mindestens zwei Größenordnungen über der des Nanosekundenlasers. Dennoch tritt die Verschiebung zu höheren Photonenenergien nur auf, wenn die Pulsenergie hinreichend hoch ist, um das Plasma aufzuheizen. Die optimale Anregung der Gasatome in Stickstoff und Krypton setzt daher sowohl hohe Pulsenergien als auch kurze Pulsdauern voraus. Li et al. [110] beobachteten eine vergleichbare Verschiebung zu höheren Photonenenergien mit zunehmender Pulsenergie für die Verwendung eines Nd:YAG-Lasers mit 150 ps Pulslänge bei Plasmen mit festem Zirkonium als Target. Die Gesamtintensität hingegen ist für Plasmen von Festkörpertargets (Zirkonium, Molybdän, Kohlenstoff und Stickstoff), die mit einem Nanosekundenlaser induziert werden, höher. Diesen Unterschied bedingen die deutlich verschiedenen Teilchendichten gasförmiger und fester Targets. Um die Anregung des Plasmas in gasförmigem Stickstoff und Krypton besser zu verstehen, wurde daher der Anteil absorbierter Laserenergie bestimmt. Dazu wurde mit einem pyroelektrischen Messkopf (SpectroLas, gentec ED-200) die Laserenergie gemessen, die vom Plasma reflektiert bzw. durch das Plasma transmittiert wird. Für beide Gase werden weniger als $2 \%$ der Laserenergie am Plasma reflektiert. Diese Beobach-

Tabelle 2.2: Anteile der Elektronentemperaturen $T_{e}$ und Elektronendichten $n_{e}$ zur Berechnung der Argonspektren

\begin{tabular}{|c|c|c|c|c|c|c|c|c|}
\hline \multirow[b]{2}{*}{$\mathrm{T}_{\mathrm{e}}[\mathrm{eV}]$} & \multicolumn{5}{|c|}{ Nanosekundenlaser } & \multicolumn{3}{|c|}{ Pikosekundenlaser } \\
\hline & 66,25 & 61,25 & 51,25 & 47,50 & 35,00 & 72,00 & 58,00 & 36,00 \\
\hline Mittelwert & \multicolumn{5}{|c|}{50,3} & \multicolumn{3}{|c|}{66,3} \\
\hline$n_{e}\left[10^{19} \mathrm{e} / \mathrm{cm}^{3}\right]$ & 2,3 & 2,7 & 7,4 & 22,9 & 3,2 & 22,9 & 22,9 & 12,1 \\
\hline Mittelwert & \multicolumn{5}{|c|}{7,0} & \multicolumn{3}{|c|}{22,4} \\
\hline Anteil [\%] & 18 & 5 & 44 & 11 & 22 & 68 & 27 & 5 \\
\hline
\end{tabular}



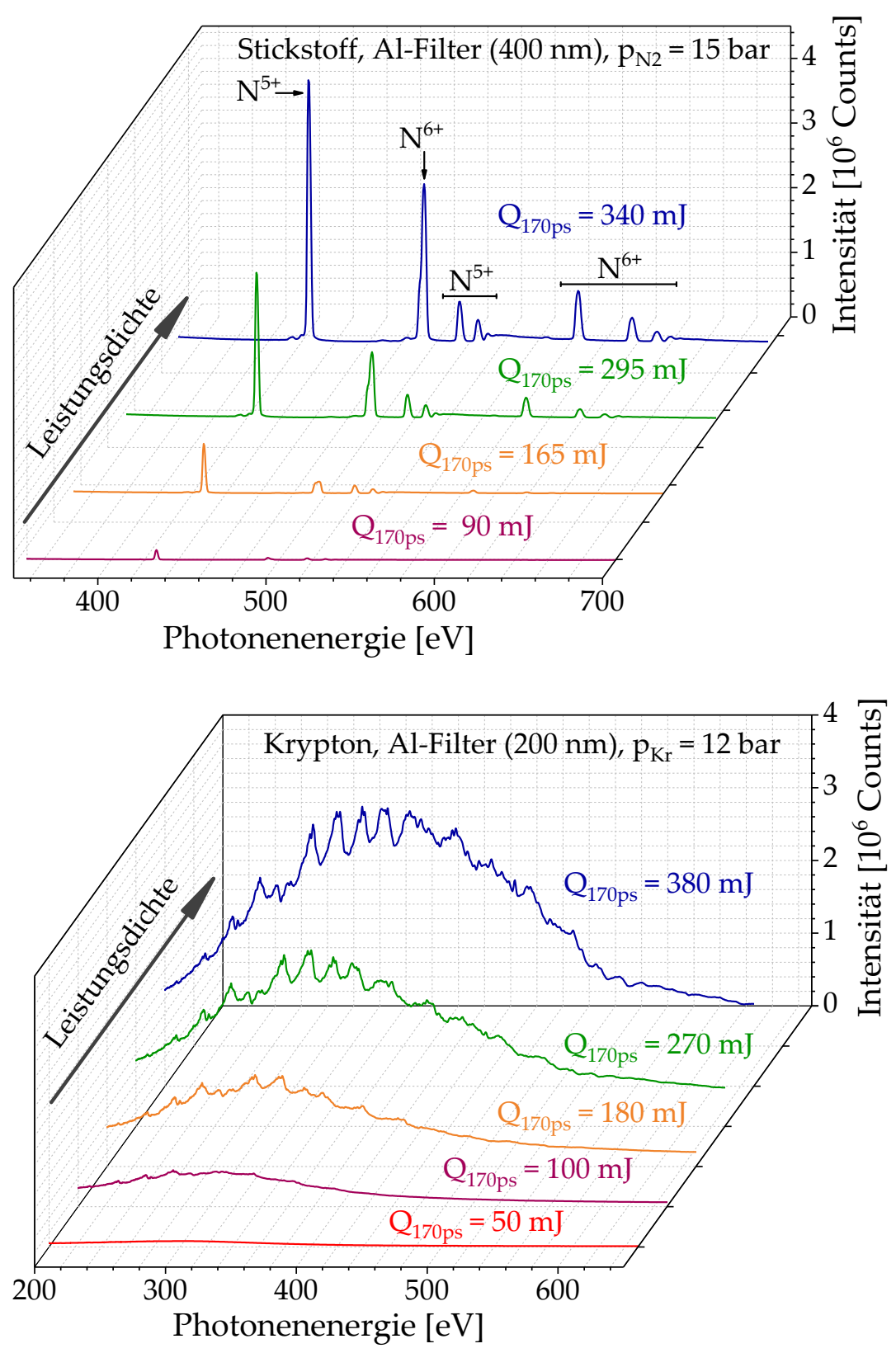

Abbildung 2.13: Emissionsspektren (100 Pulse) von Stickstoff (oben) und Krypton (unten) für verschiedene Pulsenergien des Pikosekundenlasers 
tung passt $\mathrm{zu}$ den berechneten Elektronendichten, die Werte von $10^{19}$ bis $10^{20} \mathrm{e} / \mathrm{cm}^{3}$ (siehe Tab. 2.2) annehmen und damit unterhalb der kritischen Elektronendichte von $10^{21} \mathrm{e} / \mathrm{cm}^{3}$ (siehe Abs. 2.3.1) liegen, ab der der Laserstrahl am Plasma total reflektiert wird.

Der Anteil absorbierter Laserenergie ist für Stickstoff und Krypton in Abbildung 2.14 gezeigt. Während für Krypton bis zu 95\% der Energie des Pikosekundenlasers im Plasma absorbiert werden, sind es bei dem Nanosekundenlaser lediglich 55\%. Auch wenn der Anteil für Stickstoff insgesamt geringer ist als für Krypton, zeigt sich doch die gleiche Tendenz: Im Vergleich zum Nanosekundenlaser verdoppelt sich bei maximaler Pulsenergie der Anteil absorbierter Energie für den Pikosekundenlaser auf etwa $60 \%$.

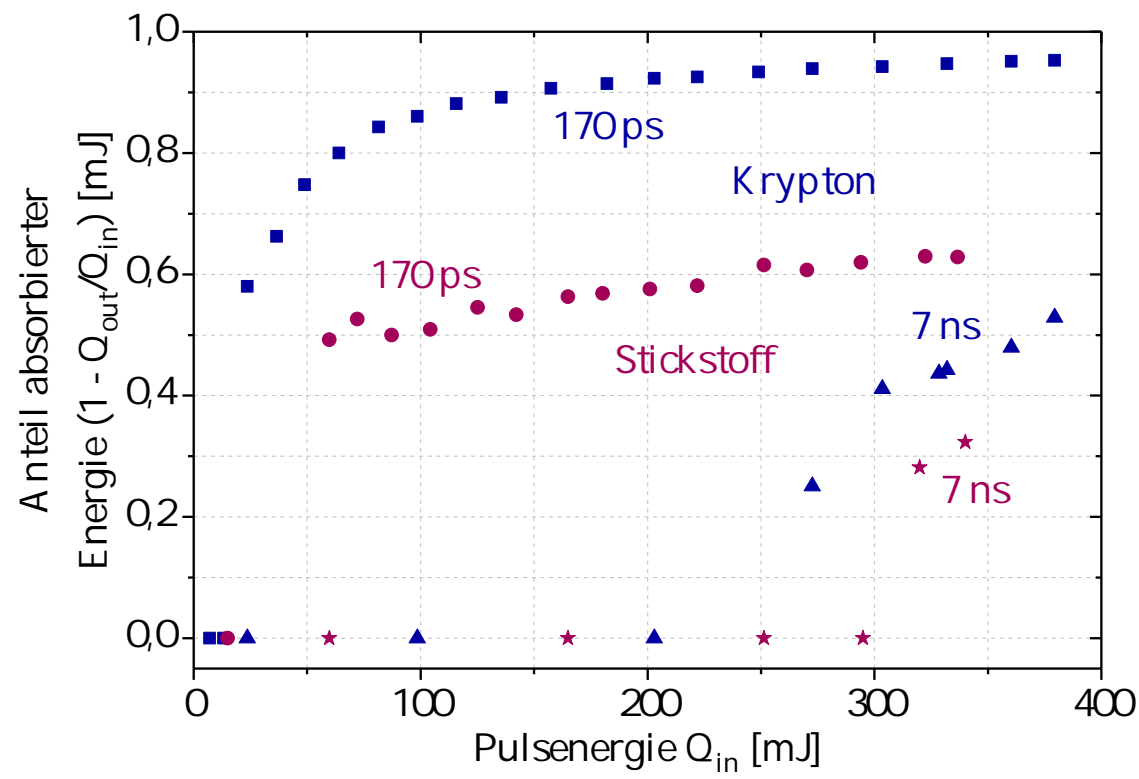

Abbildung 2.14: Anteil der absorbierten Laserpulsenergie in Stickstoff und Krypton (Gasdruck 12 bar) für Nano- und Pikosekundenlaser

Eine mögliche Ursache für die unterschiedlich starke Absorption sind Cluster erhöhter Dichte, die infolge des abrupten Abkühlens des Gases bei der adiabatischen Expansion in das Vakuum entstehen [11I]. Allerdings liegt die Zerfallszeit der Cluster im Bereich einiger weniger Pikosekunden [112], die selbst bei einer Pulslänge von 170 ps für eine effektive Wechselwirkung zwischen Clustern und Laserpuls zu kurz sein könnte. Trotzdem lässt sich die erhöhte Gasdichte der zerfallenden Cluster während der steigenden Flanke des Pikosekundenlaserpulses nutzen, um die Konversionseffizienz von Laserenergie in weiche Röntgenstrahlung zu erhöhen und dementsprechend die Brillanz der Quelle zu steigern. Der Nanosekundenlaser wechselwirkt jedoch nicht mit den Clustern, sondern mit dem rein gasförmigen Target. Dies führt zu einer verringerten Absorption der Laserenergie und damit zu einer niedrigeren Elek- 
tronentemperatur im Plasma, einem geringeren Ionisationsgrad und entsprechend einer verminderten Emission weicher Röntgenstrahlung.
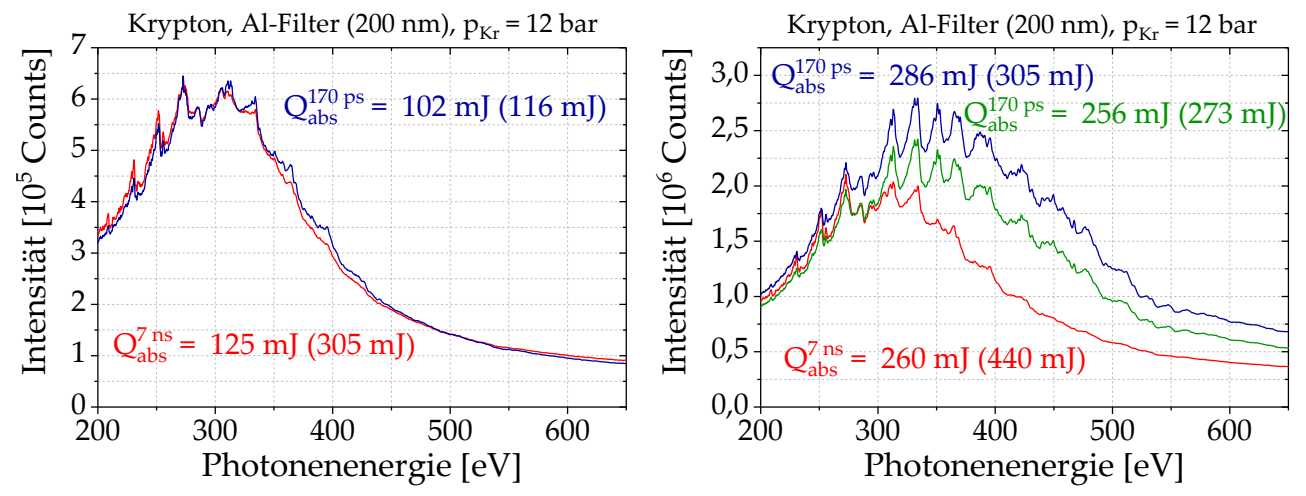

Abbildung 2.15: Emissionsspektren (100 Pulse) von Krypton in Abhängigkeit der absorbierten Energie $Q_{a b s}$ von Nano- und Pikosekundenlaser. Die dazugehörigen Laserpulsenergien sind in Klammern angegeben.

Unter Berücksichtigung der tatsächlich absorbierten Anteile lassen sich Spektren von Krypton bei annähernd gleicher Energie $\mathrm{Q}_{\mathrm{abs}}$, aber verschiedener Pulslänge vergleichen (siehe Abb. 2.15). Auch wenn sich die Leistungsdichten der beiden Laser deutlich unterscheiden, sind die Emissionsspektren für vergleichbare, kleine Werte der absorbierten Energie $\left(Q_{a b s}^{7 n s}=125 \mathrm{~mJ}\right.$ bzw. $\left.Q_{a b s}^{170 p s}=102 \mathrm{~mJ}\right)$ nahezu identisch. Erst für absorbierte Energien $\mathrm{Q}_{\mathrm{abs}}>200 \mathrm{~mJ}$ sind die Spektren unter Verwendung des Pikosekundenlasers deutlich zu höheren Photonenenergien verschoben und weisen zudem eine höhere Intensität auf. Das Plasma wird demnach aufgrund der kürzeren Pulslänge stärker aufgeheizt und die Kryptonatome werden höher angeregt. Diese Beobachtung unterstreicht, dass für eine ideale Anregung der Targetgase und entsprechend brillante Plasmen bei der Wahl des Lasers neben der kurzen Pulsdauer auch auf eine hinreichend hohe Pulsenergie $(>50 \mathrm{~mJ}) \mathrm{zu}$ achten ist.

Um eine optimale Wechselwirkung des Laserstrahls mit den Clustern zu erreichen, ist aufgrund der Zerfallszeit der Cluster eine Pulslänge von etwa 1 bis 10 ps anzustreben. Aktuell sind kompakte Lasersysteme mit diesen Parametern jedoch nicht kommerziell verfügbar. Allerdings wird am HiLASE Centre (Institut der Physik der Akademie der Wissenschaft Tschechien) gegenwärtig ein Lasersystem (Pulslänge 2 ps, Pulsenergie bis zu $500 \mathrm{~mJ}$, Repetitionsrate $1 \mathrm{kHz}$ ) entwickelt, das diese Anforderungen erfüllt. Im Rahmen einer bereits bestehenden Kooperation soll dieser Prototyp zukünftig zur Erzeugung eines Plasmas getestet werden.

\section{GASDRUCK}

Wie im vorherigen Abschnitt im Zusammenhang mit der Clusterbildung beschrieben, wird die Brillanz der laserinduzierten Plasmaquelle maßgeblich von der Teilchendichte des Targets beeinflusst. Typische 
Gasdrücke zum Betrieb der Plasmaquelle lagen bisher im Bereich von 10 bis 20 bar. Um deutlich höhere Gasdrücke bis 200 bar zu ermöglichen, wurde von Jonathan Holburg [113] im Rahmen seiner Bachelorarbeit eine neue Düse konzipiert. Im Folgenden wird die Abhängigkeit der Plasmaintensität vom Stickstoff- bzw. Kryptongasdruck bei Verwendung dieser Hochdruckdüse beschrieben; dabei wurde zur Erzeugung der Plasmen der Nano- und der Pikosekundenlaser eingesetzt.

In Abbildung 2.16 sind für beide Gase und Laser die Intensitäten der Plasmen für verschiedene Gasdrücke aufgetragen: Während für Stickstoff die Intensität aufgrund der höheren Teilchendichte im Laserfokus mit steigendem Gasdruck zunimmt, stagniert die Intensität für Krypton je nach verwendetem Laser ab einem Druck von etwa 30 bzw. 40 bar. Gleichzeitig lässt sich eine Zunahme des reflektierten Laserlichtes feststellen, da im Plasma bei diesen Drücken die kritische Elektronendichte von $10^{21} \mathrm{e} / \mathrm{cm}^{3}$ erreicht und das Plasma nicht weiter aufgeheizt wird. Die Intensität nimmt deshalb auch für höhere Drücke nicht weiter zu. Unter Verwendung des Pikosekundenlasers werden die Kryptonatome stärker ionisiert (siehe Abs. 2.3.4). Der Laserstrahl wird daher bereits bei einem niedrigeren Druck als für den Nanosekundenlaser am Plasma reflektiert. Aufgrund der geringeren Anzahl an Elektronen wird in Stickstoff die kritische Elektronendichte auch bei Gasdrücken bis 100 bar noch nicht erreicht.
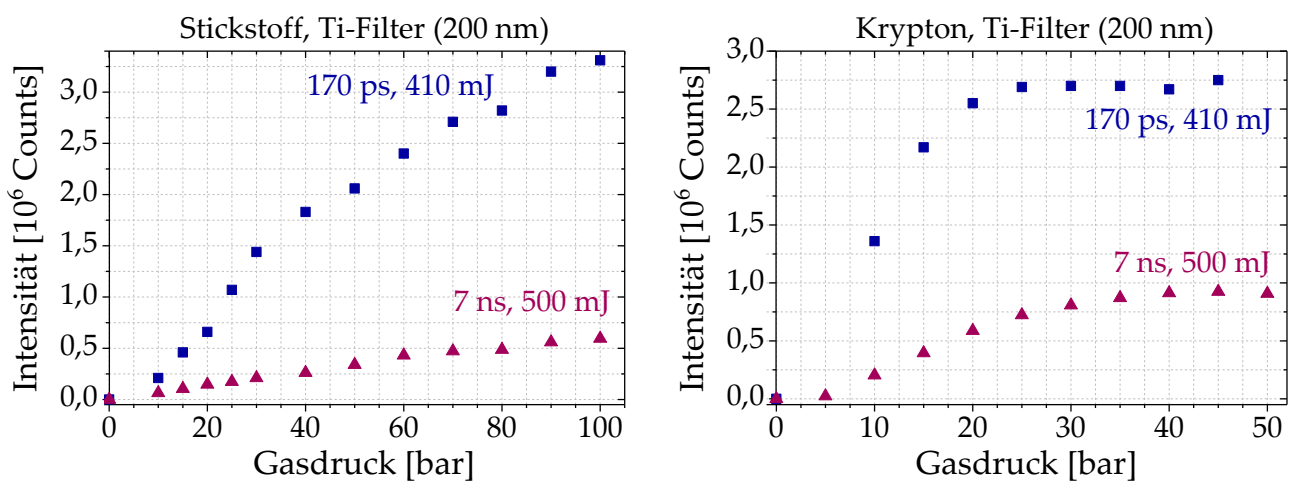

Abbildung 2.16: Intensität (Mittelung 100 Pulse) von Stickstoff- (links) und Kryptonplasma (rechts) in Abhängigkeit vom Gasdruck (Abstand Plasma Düse $1 \mathrm{~mm}$ außer für Stickstoff und Nanosekundenlaser, hier: $550 \mu \mathrm{m}$; Verstärkungsfaktor Lochkamera 6 für Stickstoff und 1 für Krypton)

Eine weitere Erhöhung des Drucks ist in Kombination mit dem Pikosekundenlaser allerdings problematisch, da das Düsenplättchen stark beschädigt wird (siehe Abb. 2.17). Die abgetragene Fläche befindet sich bezogen auf die Ausbreitungsrichtung des Laserstrahls hinter der Düsenöffnung. Daraus lässt sich schließen, dass erstens das Plasma bereits bei der steigenden Flanke des Laserpulses gezündet wird, und zweitens der Anteil nicht absorbierter Laserstrahlung vom Plasma verformt wird. Der Laserstrahl wird infolgedessen stark aufgeweitet und abla- 


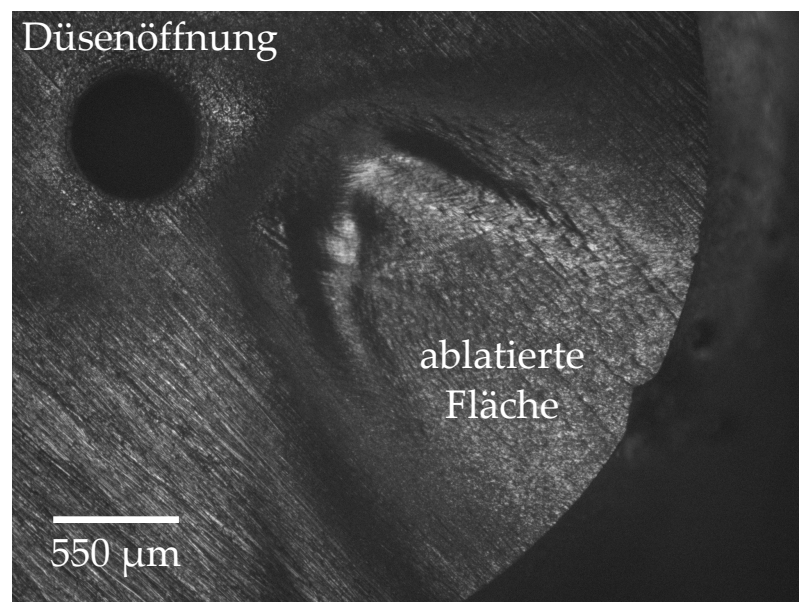

Abbildung 2.17: Mikroskopaufnahme (Hellfeld, 5fache Vergrößerung) eines durch Pikosekundenlaserstrahlung beschädigten Düsenplättchens. Der Laser breitet sich von oben links nach unten rechts aus; die ablatierte Fläche befindet sich demnach hinter der Düsenöffnung.

tiert Material von dem Düsenplättchen. Folglich setzt sich Wolfram in der Vakuumkammer u. a. auf dem Laserein- und -austrittsfenster sowie der dünnen Titanfolie ab, sodass deren Transmittivitäten zunehmend reduziert werden. Um die Zerstörung der Bauteile zu verhindern, könnte der Abstand zwischen Düse und Plasma auf deutlich über $1 \mathrm{~mm}$ erhöht werden, bis der Laserstrahl das Düsenplättchen nicht mehr erreicht. Allerdings würde sich dadurch die Intensität des Plasmas stark reduzieren. Alternativ könnte die Geometrie der Düse entsprechend verändert werden, um eine Ablation des Wolframs zu vermeiden und einen debrisfreien Betrieb der laserinduzierten Plasmaquelle sicherzustellen. Eine dritte Möglichkeit wäre es, andere Materialien wie Borcarbid, Tantalcarbid, Titanborid oder Aluminiumoxid zu verwenden, die möglicherweise gar nicht oder nicht so stark abgetragen werden wie Wolfram.

\section{WIN KELABH ÄNGIGKEIT}

Aus den Referenzen [114, 115] ist bekannt, dass weiche Röntgenstrahlung in der Ebene senkrecht zur Düsenachse nicht isotrop abgestrahlt wird: Die Emission ist abhängig vom Winkel zwischen einfallender Laserstrahlung und Beobachtungsposition des Plasmas. Da das Plasma asymmetrisch zur Düsenachse gezündet wird, legt die emittierte Strahlung unterschiedlich lange Wegstrecken durch das Gas zurück. Je größer der Winkel und damit der Weg durch das Gasvolumen ist, desto stärker wird die emittierte Strahlung absorbiert. Kranzusch et al. [115] haben für azimutale Winkel von 40 bis $130^{\circ}$ eine Abnahme der Intensität um den Faktor 8 gemessen. Allerdings verwendeten sie eine zylindrische Düse, die im Vergleich zu der in dieser Arbeit benutzten konisch geformten Düse einen weniger stark gerichteten Gasstrahl produziert. Zudem wur- 
den die Experimente bei einem Gasdruck von lediglich 1,75 bar durchgeführt. Inwiefern sich ein höherer Gasdruck von 25 bar auf Größe und Emissionseigenschaften des Plasmas unter verschiedenen Winkeln auswirken, wird im folgenden Absatz beschrieben.

Die Versuchsanordnung zur Untersuchung der Winkelabhängigkeit ist in Abbildung 2.18 gezeigt. Der Laserstrahl (Pulslänge $7 \mathrm{~ns}$, Pulsener-
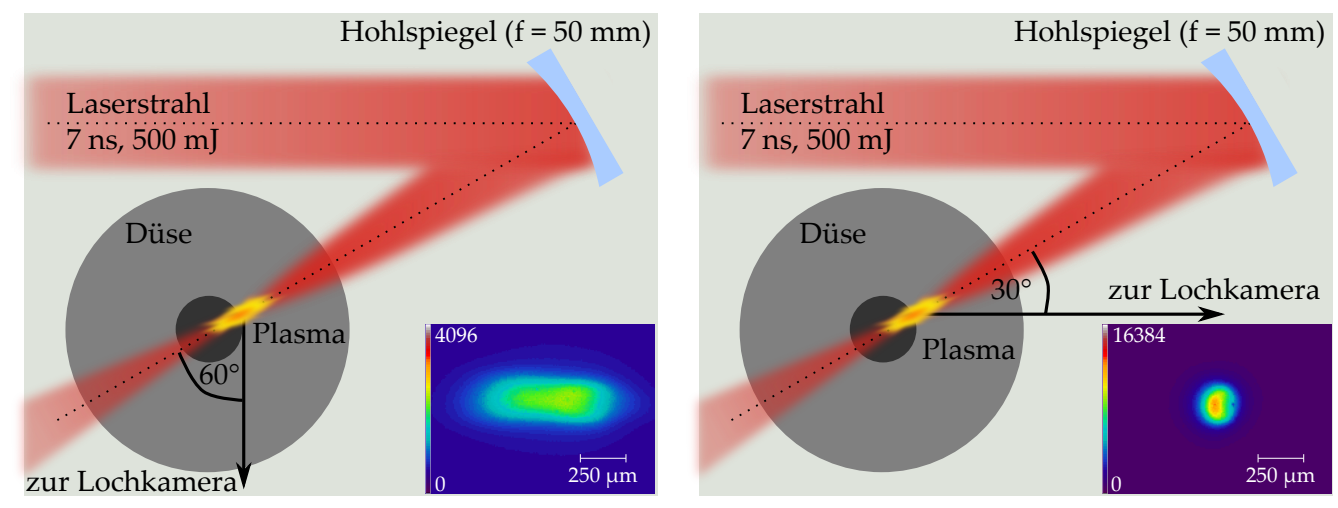

Abbildung 2.18: Schematische Darstellung (nicht maßstabsgerecht) des Aufbaus zur Plasmacharakterisierung unter $120^{\circ}$ (links) und $30^{\circ}$ (rechts) zum einfallenden Laserstrahl und entsprechende Lochkamerabilder der Kryptonplasmen (Mittelung 100 Pulse, Gasdruck 25 bar, Abstand zur Düse $550 \mu \mathrm{m}$ ) im Spektralbereich weicher Röntgenstrahlung.

gie $500 \mathrm{~mJ}$ ) wird mit einem Hohlspiegel (Brennweite $50 \mathrm{~mm}$ ) auf den Gasstrahl (Krypton, Gasdruck 25 bar) fokussiert. Zur Charakterisierung des Plasmas wird eine Lochkamera unter $120^{\circ} \mathrm{bzw} .30^{\circ} \mathrm{zum}$ einfallenden Laserstrahl eingesetzt. Anhand der Plasmabilder in Abbildung 2.18 lässt sich erkennen, dass das Plasma für den kleineren Winkel sowohl signifikant heller als auch kleiner geworden ist (siehe Tabelle 2.3). So nimmt die Gesamtenergie um den Faktor 1,6 zu, da die emittierte Strahlung über das Plasmavolumen integriert wird. Gleichzeitig verringert sich die Plasmafläche auf ein Viertel. Die Brillanz der Plasmaquelle erhöht sich demnach etwa um den Faktor 6. Zudem ist das Plasma nicht mehr elliptisch, sondern annähernd kreisförmig.

Mit der Lochkamera lassen sich Intensität und Größe des Plasmas sowie dessen Lagestabilität bestimmen. Dazu wird für jede Plasmaabbildung der Intensitätsschwerpunkt ermittelt und anschließend werden der Mittelwert aller Schwerpunkte sowie die Standardabweichung berechnet. In Abbildung 2.19 sind für beide Winkel jeweils die Schwerpunkte von 100 Kryptonplasmen aufgetragen. Dichteschwankungen im Gasstrom führen dazu, dass das Plasma in Ausbreitungsrichtung des Laserstrahls an unterschiedlichen Stellen gezündet wird. Unter einem Beobachtungswinkel von $120^{\circ}$ schwankt der Plasmaschwerpunkt daher entlang dieser Richtung um $\sigma_{x}^{120^{\circ}} \approx 10 \mu \mathrm{m}$. Wird der Beobachtungswin- 
kel verringert, nimmt die Lagestabilität des Plasmas speziell in Laserstrahlrichtung fast um den Faktor $8 \mathrm{zu}$ (siehe Tab. 2.3).

Tabelle 2.3: Vergleich charakteristischer Größen des Kryptonplasmas, gemessen mit Lochkamera unter $120^{\circ}$ und $30^{\circ}$ zum einfallenden Laserstrahl

\begin{tabular}{lcc|c}
\hline & $120^{\circ}$ & $30^{\circ}$ & Faktor \\
\hline Maximum $\left[10^{3}\right.$ Counts] & 1,83 & 12,83 & 6,8 \\
Gesamtenergie $\left[10^{6}\right.$ Counts] & 5,47 & 8,67 & 1,6 \\
Größe $\left(1 / \mathrm{e}^{2}\right)[\mathrm{mm} \times \mathrm{mm}]$ & $0,94 \times 0,35$ & $0,27 \times 0,29$ & $0,29 \times 0,83$ \\
Fläche $\left[\mathrm{mm}^{2}\right]$ & 0,26 & 0,06 & 0,23 \\
Exzentrizität $[-]$ & 0,38 & 0,93 & 2,45 \\
Ortsstabilität $\sigma_{x}[\mu \mathrm{m}]$ & 10,1 & 1,34 & 0,13 \\
Ortsstabilität $\sigma_{y}[\mu \mathrm{m}]$ & 1,0 & 0,7 & 0,7 \\
\hline
\end{tabular}

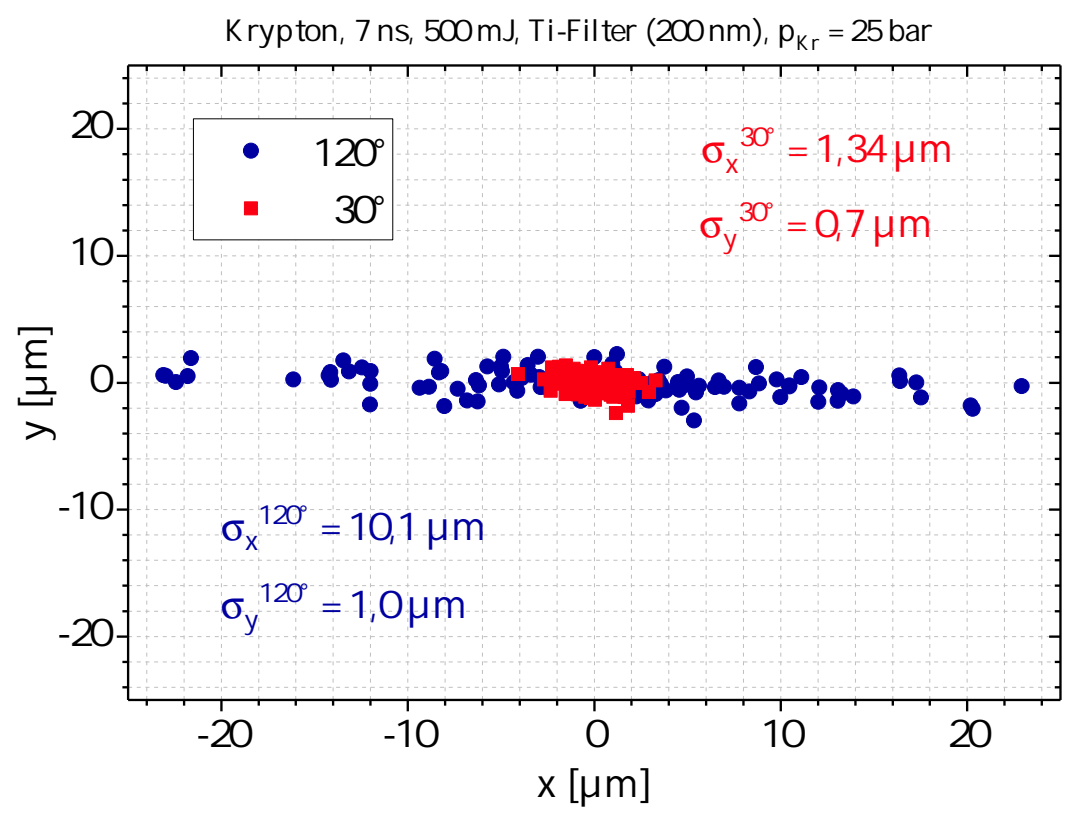

Abbildung 2.19: Lage und Standardabweichung der Schwerpunkte von 100 Kryptonplasmen unter einem Winkel von $30^{\circ}$ bzw. $120^{\circ}$. 


\subsubsection{OPTIMIERUNG DER PLASMAQUELLE}

Im Rahmen dieser Arbeit wurden verschiedene Maßnahmen vorgestellt (siehe Abs. 2.3.4), mit denen sich die Brillanz der laserinduzierten Plasmaquelle mit gasförmigem Target insgesamt um bis zu zwei Größenordnungen steigern lässt. Darüber hinaus gibt es jedoch weitere Möglichkeiten zur Optimierung der Quelle. So sollte die Ausdehnung des Plasmas verringert werden, denn im Vergleich zu Plasmaquellen mit festen oder flüssigen Targets ist das hier beschriebene Plasma etwa um einen Faktor 10 größer. Dazu könnten Düsen mit entsprechend 10fach kleineren Öffnungsdurchmessern getestet werden. Die damit einhergehende Verringerung der Gasteilchendichte ließe sich z. B. durch einen höheren Gasdruck kompensieren.

Die mittlere Brillanz wird außerdem durch die geringen Repetitionsraten von lediglich $5 \mathrm{~Hz}$ der vorhandenen Laser begrenzt. Mit neueren Systemen sind bei vergleichbaren Pulsenergien und -längen hingegen 20 bis $100 \mathrm{~Hz}$ möglich. Mit zunehmender Wiederholrate steigt allerdings der Gasballast stark an und die weiche Röntgenstrahlung wird reabsorbiert. Mit einer differenziellen Pumpstufe kann die Quellkammer aber vom übrigen Vakuumsystem getrennt und die Absorption der Strahlung minimiert werden. Eine Steigerung der Repetitionsrate auf mindestens $25 \mathrm{~Hz}$ sollte damit möglich sein, sodass die mittlere Brillanz der Plasmaquelle auf das Fünffache erhöht wird.

Unabhängig davon sollte die Teilchendichte insbesondere in größerer Entfernung $(>500 \mu \mathrm{m})$ zur Düse gesteigert werden. Statt einer Düse könnten hierfür zwei Düsen verwendet werden, die sich im Abstand weniger Millimeter gegenüberstehen. Das Gas expandiert dadurch ähnlich wie bei der Doppelstrahldüse von Fiedorowicz et al. [104] oder dem Fassstoß von Mey et al. [105] nicht ungehindert ins Vakuum, da es auf den Gaspuls der jeweils anderen Düse trifft. Die Auswirkung dieser Wechselwirkung auf die Gasverteilung zwischen den Düsen wurde mithilfe des Schlierenverfahrens untersucht. Dabei handelt es sich um eine gängige Methode aus der Fluiddynamik, mit der qualitativ Dichtegradienten visualisiert werden können [116]. Der Aufbau zur Aufnahme von Schlierenabbildungen ist z. B. in Referenz [105] beschrieben. Für die Messungen wurde an die eine Düse Stickstoff und an die andere Düse Krypton angelegt. Der Abstand zwischen den Düsen beträgt $2 \mathrm{~mm}$. Abbildung 2.20 zeigt die entsprechenden Schlieren- und dazugehörigen Plasmaaufnahmen für die Nutzung einer bzw. gleichzeitig beider Düsen. Wird zur Krypton- auch die Stickstoffdüse zugeschaltet, manifestiert sich zwischen den Düsen ein großer Dichtegradient (siehe Abb. 2.20e), der auf einen Bereich mit höherer Teilchendichte schließen lässt. Die Gesamtenergie des Plasmas erhöht sich dadurch um den Faktor 1,6. Zudem verringert sich die Plasmafläche um etwa $45 \%$ (weitere 


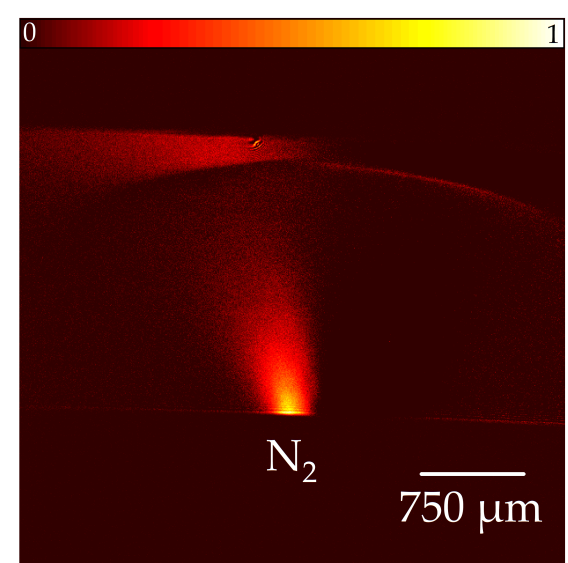

(a) $p_{N_{2}}=15$ bar

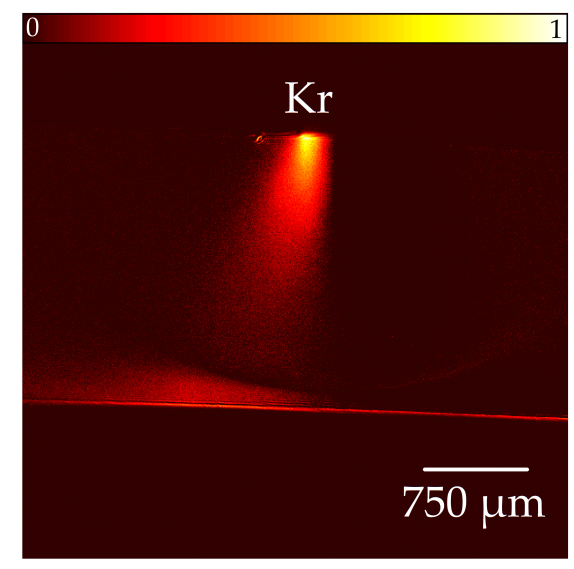

(c) $p_{\mathrm{Kr}}=10 \mathrm{bar}$

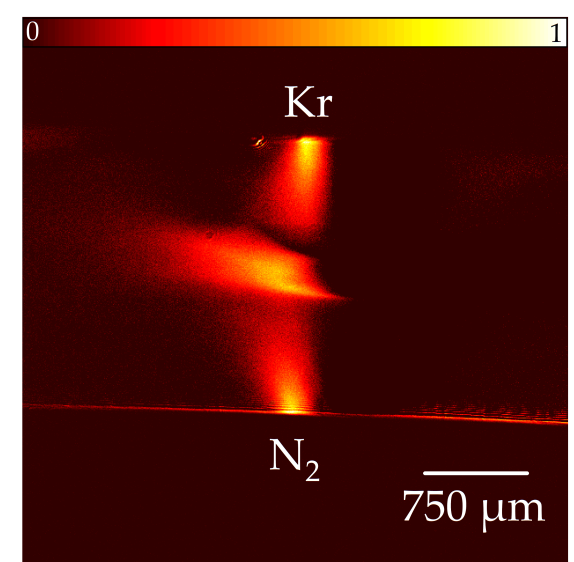

(e) $p_{\mathrm{Kr}}=10 \mathrm{bar}, p_{\mathrm{N}_{2}}=15 \mathrm{bar}$

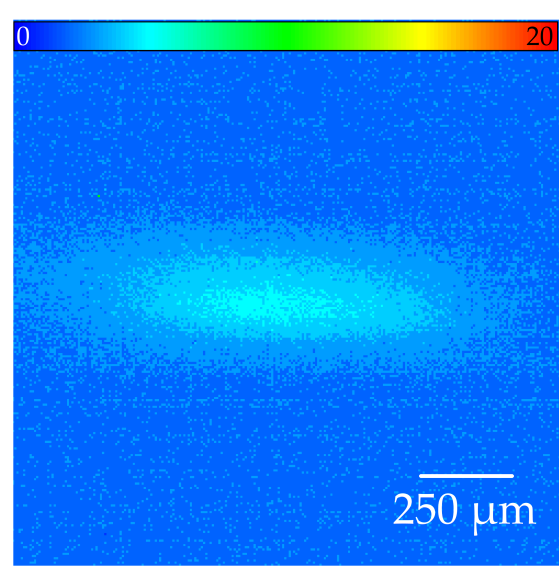

(b) $p_{N_{2}}=15 \mathrm{bar}$

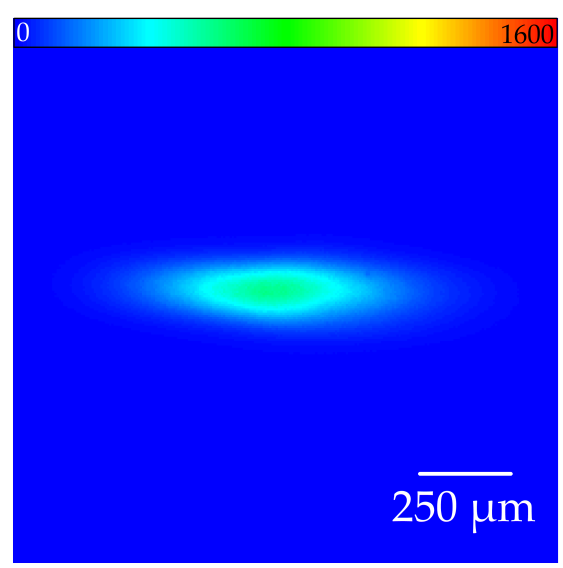

(d) $p_{\mathrm{Kr}}=10 \mathrm{bar}$

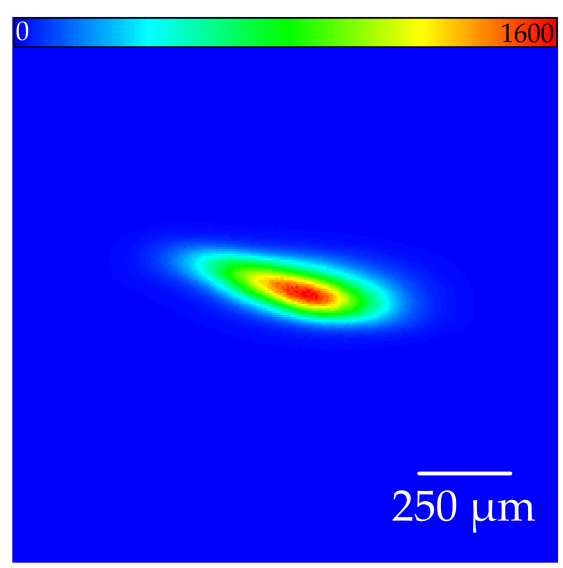

(f) $p_{\mathrm{Kr}}=10 \mathrm{bar}, \mathrm{p}_{\mathrm{N}_{2}}=15 \mathrm{bar}$

Abbildung 2.20: Schlierenaufnahme zur Visualisierung des Dichtegradienten (links) und Lochkamerabilder (Mittelung 100 Pulse, Abstand Plasma - Düse $1 \mathrm{~mm}$, Spektralbereich weiche Röntgenstrahlung) der dazugehörigen Plasmen (rechts) für Krypton, Stickstoff und beide Gase zusammen. Aufgrund des großen Intensitätsunterschieds zwischen Stickstoff- und Kryptonplasma (ca. Faktor 80) wurde die Skala angepasst. 
Parameter siehe Tab. 2.4). Die Intensitätszunahme lässt sich dabei nicht auf die Emission weicher Röntgenstrahlung des zusätzlichen Stickstoffs im Laserfokus zurückführen, denn das Stickstoffplasma ist etwa 80fach dunkler als das Kryptonplasma. Die Asymmetrie der Gasdichte und die damit verbundene Neigung des Plasmas ergibt sich, da die Düsen nicht exakt senkrecht gegenüber stehen. Zudem fällt auf, dass die Lagestabilität der Plasmen um den Faktor 3 schlechter wird, wenn beide Düsen genutzt werden. Weiterführende Untersuchungen bieten allerdings die Möglichkeit zahlreiche Parameter zu variieren, um die Brillanz der Quelle weiter zu erhöhen und die Ortsstabilität wieder zu verbessern. So ist z. B. von Interesse, wie sich die Gasdichteverteilung bei Variation des Düsenabstands, höherem Gasdruck, anderen Gasen bzw. Kombinationen verschiedener Gase und unterschiedlichen Öffnungszeitpunkten der Düsen verändert.

Tabelle 2.4: Vergleich charakteristischer Größen der beiden Plasmen, aufgenommen nur mit Krypton sowie Krypton und Stickstoff zusammen

\begin{tabular}{lcc|c}
\hline & $\mathrm{Kr}$ & $\mathrm{Kr}$ und $\mathrm{N}_{2}$ & Faktor \\
\hline Maximum $\left[10^{3}\right.$ Counts $]$ & 0,6 & 1,6 & 2,7 \\
Gesamtenergie $\left[10^{6}\right.$ Counts] & 1,24 & 1,94 & 1,6 \\
Größe $\left(1 / \mathrm{e}^{2}\right)[\mathrm{mm} \times \mathrm{mm}]$ & $0,91 \times 0,23$ & $0,62 \times 0,18$ & $0,7 \times 0,8$ \\
Fläche $\left[\mathrm{mm}^{2}\right]$ & 0,16 & 0,09 & 0,56 \\
Ortsstabilität $\sigma_{x}[\mu \mathrm{m}]$ & 35 & 110 & 3,1 \\
Ortsstabilität $\sigma_{y}[\mu \mathrm{m}]$ & 5 & 16 & 3,2 \\
\hline
\end{tabular}



Bereits im Jahr 1952 postulierte Hans Wolter, dass weiche Röntgenstrahlung insbesondere zwischen den Absorptionskanten von Kohlenstoff und Sauerstoff im sogenannten "Wasserfenster" $(\lambda=2,3-4,4 \mathrm{~nm})$ zur Mikroskopie geeignet sei [117]. Dafür gibt es zwei wesentliche Gründe: Zum einen ist in diesem Spektralbereich die Eindringtiefe in kohlenstoffhaltige Proben gegenüber Wasser um etwa eine Größenordnung geringer (siehe Abb. 3.I). Daraus resultiert ein natürlicher Absorptions-

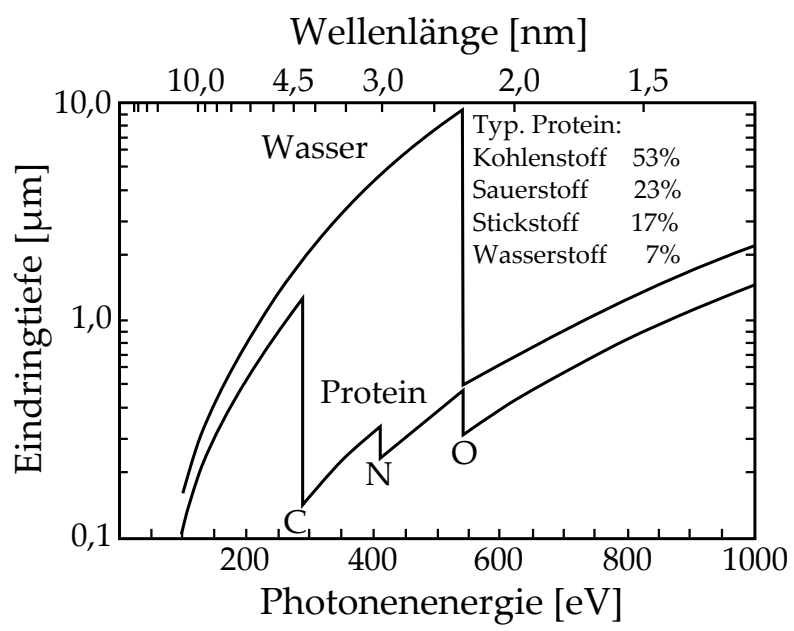

Abbildung 3.1: Eindringtiefe weicher Röntgenstrahlung in Wasser und einem typischen Protein (Dichte $\rho \approx 1,3 \mathrm{~g} \mathrm{~cm}^{-3}$ ) als Funktion der Photonenenergie (Daten aus Ref. [6])

kontrast, der u.a. für die Abbildung biologischer Proben genutzt werden kann. Zum anderen ist aufgrund der geringen Wellenlänge eine hohe räumliche Auflösung von wenigen Nanometern möglich. Doch erst in den letzten 20 Jahren hat sich die Röntgenmikroskopie durch viele erfolgreiche Anwendungen in verschiedenen Wissenschaftsdisziplinen zu einer etablierten Messmethode an Synchrotronen entwickelt [118]. Zugleich werden für Röntgenmikroskope zunehmend kompakte Laborquellen eingesetzt.

Um die Funktionsweise von Röntgenmikroskopen verstehen zu können, folgen in den nächsten Abschnitten zuerst die theoretischen Grundlagen der zugrunde liegenden Kontrastmechanismen, zum Auflösungsvermögen, zur Kohärenz und von Strahlenschäden. Zudem werden verschiedene optische Elemente z. B. Zonenplatten beschrieben, die typischerweise zur Strahlführung und -fokussierung genutzt werden. Daran 
anschließend wird der aktuelle Stand der Forschung mit Laborröntgenmikroskopen erläutert (siehe Abs. 3.2), bevor auf das Mikroskop eingegangen wird, das während dieser Arbeit aufgebaut und charakterisiert wurde (siehe Abs. 3.3).

\subsection{GRUNDLAGEN}

Die Wechselwirkung weicher Röntgenstrahlung mit Materie ist grundlegend anders als im sichtbaren Spektralbereich. Einerseits unterscheiden sich daher die Kontrastmechanismen zu anderen mikroskopischen Verfahren. Andererseits folgen daraus besondere Anforderungen an den Aufbau eines Röntgenmikroskops z. B. hinsichtlich der Auswahl geeigneter Optiken zur Strahlführung. Im Folgenden werden diese Zusammenhänge genauer erläutert.

\subsubsection{KONTRASTMECHANISMEN UND AUFLÖSUNGSVERMÖGEN}

Ein Röntgenphoton kann in Materie absorbiert, elastisch oder inelastisch gestreut werden. Dabei wird die Absorption im Energiebereich von 250 bis $1250 \mathrm{eV}$ hauptsächlich von der Wechselwirkung der einfallenden Strahlung mit den kernnahen Elektronen der Atome innerhalb des Mediums bestimmt. Für die elastische Streuung muss hingegen die gesamte Elektronendichte berücksichtigt werden. Allerdings tragen elastische Streuprozesse kaum zur Abschwächung weicher Röntgenstrahlung beim Durchdringen eines Mediums bei. Inelastische Streuprozesse und Mehrfachstreuung sind aufgrund des geringen Wechselwirkungsquerschnitts vollständig vernachlässigbar [119].

Die Ausbreitung einer elektromagnetischen Welle in Materie lässt sich für unpolarisierte Röntgenstrahlung auf makroskopischer Ebene über den komplexen Brechungsindex

$$
n=1-\delta+i \beta
$$

beschreiben. Der Realteil charakterisiert dabei die Brechung (Phasenverschiebung), während der Imaginärteil direkt proportional zum linearen Absorptionskoeffizienten $\mu=4 \pi \beta / \lambda$ des Mediums ist. In einem Material der Dicke $d$ nimmt die Intensität $\mathrm{I}_{0}$ der Strahlung mit der Wellenlänge $\lambda$ gemäß Lambert-Beer'schem Gesetz [6]

$$
\mathrm{I}=\mathrm{I}_{0} e^{-\mu \mathrm{d}}=\mathrm{I}_{0} e^{-\frac{4 \pi \beta}{\lambda} \mathrm{d}}
$$

exponentiell ab. Alternativ zur makroskopischen Beschreibung können Absorption und Streuung auf atomarer Ebene durch den komplexen Streufaktor dargestellt werden. Dieser gibt das Amplitudenverhältnis zweier elektromagnetischer Wellen an, die an einem Atom bzw. freien 
Elektron gestreut werden. Für Röntgenstrahlung ist im Falle der Vorwärtsstreuung der atomare Streufaktor mit dem Brechungsindex verknüpft [6]:

$$
n=1-\frac{n_{a} r_{e} \lambda^{2}}{2 \pi}\left(f_{1}^{0}-i f_{2}^{0}\right)
$$

Dabei bezeichnet $n_{a}$ die atomare Dichte, $r_{e}$ den klassischen Elektronenradius, $\lambda$ die Wellenlänge der Röntgenstrahlung und $f_{1}^{0}$ sowie $f_{2}^{0}$ den Real- bzw. Imaginärteil des atomaren Streufaktors. Aus dem Vergleich von Gl. (3.1) und (3.3) folgt damit für Brechung und Absorption:

$$
1-\delta=1-\frac{n_{a} r_{e} \lambda^{2}}{2 \pi} f_{1}^{0} \quad \text { und } \quad \beta=\frac{n_{a} r_{e} \lambda^{2}}{2 \pi} f_{2}^{0} .
$$

Für die Elemente mit Ordnungszahl von 1 bis 92 sind $f_{1}^{0}$ und $f_{2}^{0}$ über einen großen Spektralbereich tabelliert [120]. So beträgt etwa für Kohlenstoff $(Z=6)$ bei einer Energie von $E=430 \mathrm{eV}$ der Realteil $f_{1}^{0}=$ 5,74 und der Imaginärteil $f_{2}^{0}=2,45$. Daraus folgt nach Gleichung (3.4) $\delta=2,4 \times 10^{-3}$ und $\beta=1,0 \times 10^{-3}$. Der Realteil des Brechungsindex $\operatorname{Re}(n)=1-\delta$ ist für weiche Röntgenstrahlung demnach nur geringfügig kleiner als Eins. Aus dem energieabhängigen Verlauf der Streufaktoren $f_{1}^{0}$ und $f_{2}^{0}$ für Kohlenstoff (siehe Abb. 3.2) werden zudem zwei weitere generelle Eigenschaften deutlich: Außer an den Absorptionskanten ist $f_{1}^{0}$ nahezu konstant und entspricht oberhalb der Kante ungefähr der Ordnungszahl Z des Elements, der Imaginärteil $f_{2}^{0}$ hingegen skaliert mit $E^{-2}$. Gemäß dem Lambert-Beer'schen Gesetz (siehe Gl. (3.2)) nimmt die Probendicke $d_{e}=1 / \mu$, die zu einer Abnahme der Intensität $I_{0}$ auf das 1 /e-fache führt, daher mit der dritten Potenz der Photonenenergie zu. Das heißt, mit weicher Röntgenstrahlung können Proben sowohl mit Absorptions- als auch Phasenkontrastverfahren abgebildet werden. Für höhere Photonenenergien ab etwa $10 \mathrm{keV}$ wird die Röntgenstrahlung bei gleicher Probendicke hingegen kaum absorbiert, und der Bildkontrast entsteht fast ausschließlich infolge der Phasenänderung.

Da im Absorptionskontrast nur die transmittierte Intensität gemessen werden muss, ist dieses Abbildungsverfahren im Bereich weicher Röntgenstrahlung vor allem bei Labormikroskopen die am häufigsten eingesetzte Methode. Verbreitet ist zudem die Dunkelfeldmikroskopie, bei der nur an der Probe gestreutes Licht detektiert wird. Sie eignet sich vor allem zur Abbildung sehr dichter Objekte, da diese stark streuen.

An synchrotronbasierten Röntgenmikroskopen werden darüber hinaus weitere Verfahren wie differenzieller Phasenkontrast, Fluoreszenzkontrast, Phasenkontrast nach Zernike und differenzieller Interferenzkontrast nach Nomarski genutzt. Eine ausführliche Übersicht über diese Methoden und deren Anwendungen findet sich z. B. in Referenz [13].

Um die Qualität eines Mikroskops unabhängig vom Aufnahmeverfahren bewerten zu können, werden meist dessen räumliches Auflösungs- 
vermögen und der Kontrast der Abbildung ermittelt. Zur Definition des Kontrastes gibt es mehrere mögliche Ansätze. In der Mikroskopie wird häufig der Michelson-Kontrast als relativer Intensitätsunterschied zwischen dem hellsten und dunkelsten Punkt des Bildes verwendet [121]:

$$
\mathrm{C}_{M}=\frac{\mathrm{I}_{\max }-\mathrm{I}_{\min }}{\mathrm{I}_{\max }+\mathrm{I}_{\min }}
$$

Für eine Absolutmessung des Kontrastes ist diese Definition allerdings nicht geeignet, denn bereits einige wenige extrem helle oder dunkle Bildpunkte würden das Ergebnis verfälschen. Zur Bestimmung von maximaler und minimaler Intensität sollte daher lokal über mehrere Pixel innerhalb des relevanten Bildbereiches gemittelt werden.

Eng verknüpft mit dem Bildkontrast ist das Signal-Rausch-Verhältnis SNR (engl. signal-to-noise ratio). Es beschreibt die Empfindlichkeit eines bildgebenden Systems, indem die mittlere Intensität $\overline{\mathrm{I}}$ der Abbildung mit der Standardabweichung des Rauschens $\sigma_{\mathrm{bg}}$ aus einer Dunkelbildaufnahme verglichen wird:

$$
\mathrm{SNR}=\frac{\text { Signal }}{\text { Rauschen }}=\frac{\overline{\mathrm{I}}}{\sigma_{\mathrm{bg}}} .
$$

Der Informationsgehalt einer Aufnahme wird außer vom Kontrast auch durch das Auflösungsvermögen des Mikroskops bestimmt. Es be-
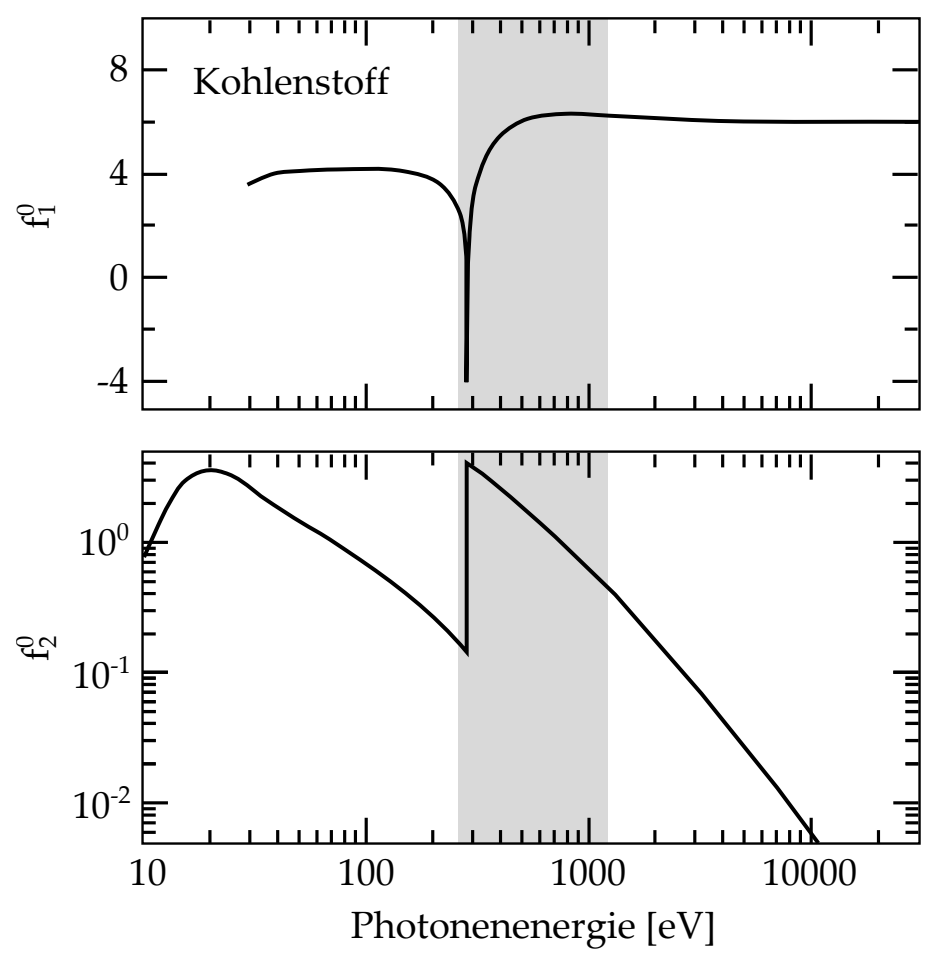

Abbildung 3.2: Verlauf von $f_{1}^{0}$ und $f_{2}^{0}$ für Kohlenstoff als Funktion der Photonenenergie (Daten aus Ref. [120]). Der für diese Arbeit relevante Bereich weicher Röntgenstrahlung ist grau hinterlegt. 
zeichnet den minimalen Abstand zweier Strukturen, um diese im Bild noch getrennt wahrnehmen zu können. Dieser Abstand wird z. B. ermittelt, indem die Airy-Scheibchen von zwei monochromatischen und zueinander inkohärenten Punktlichtquellen eines Objekts betrachtet werden. Wenn das Maximum des einen Beugungsscheibchens in das erste Minimum des anderen fällt, dann sind die beiden Objekte nach Rayleigh zueinander aufgelöst [122]. Für den minimalen Abstand gilt demnach

$$
\delta^{\text {Rayl. }}=0,61 \frac{\lambda}{\mathrm{NA}}
$$

wobei NA die numerische Apertur der abbildenden Optik bezeichnet.

Die kurze Wellenlänge weicher Röntgenstrahlung ermöglicht bei hinreichend hoher NA eine theoretische Auflösung von wenigen Nanometern. Aktuell ist sie allerdings nicht durch die Wellenlänge, sondern aufgrund der verfügbaren Optiken (siehe Abs. 3.1.2) auf etwa $10 \mathrm{~nm}$ begrenzt [123]. Experimentell lässt sich die räumliche Auflösung z. B. bestimmen, indem der Intensitätsverlauf einer kontrastreichen Kante im Bild vermessen wird (,Knife-Edge-Test“). Der Abstand $\delta^{\text {Rayl. }}$ entspricht dabei ungefähr der Intensitätsabnahme von $90 \%$ auf $10 \%$ [6].

Bisher wurden Bildkontrast und Auflösung als voneinander unabhängige Größen definiert. Das Auflösungsvermögen eines Mikroskops kennzeichnet aber nicht nur die Fähigkeit zwei Objektstrukturen räumlich getrennt abzubilden, sondern auch verschiedene Ortsfrequenzen aus der Bild- in die Detektorebene zu übertragen. Bei inkohärenter Beleuchtung wird dies durch die optische Übertragungsfunktion OTF (engl. optical transfer function) beschrieben [124]:

$$
\operatorname{OTF}(\mathbf{u})=\operatorname{MTF}(\mathbf{u}) e^{i \cdot \operatorname{PTF}(\mathbf{u})} .
$$

Dabei umfasst die Phasenübertragungsfunktion PTF (engl. phase transfer function) alle Änderungen der Phase. Der Betrag der OTF hingegen charakterisiert den Kontrastübertrag verschiedener Raumfrequenzen $\mathbf{u}$ aus der Objekt- in die Bildebene und wird Modulationsübertragungsfunktion MTF (engl. modulation transfer function) genannt. Die MTF verknüpft damit den Kontrast eines Bildes mit der Auflösung und beinhaltet zudem mögliche Aberrationen des Mikroskops.

Die MTF eines abbildenden Systems lässt sich direkt oder indirekt ermitteln. Direkte Verfahren basieren auf dem Vergleich von Bild- und Objektkontrast. Als ideales Objekt eignet sich hierfür ein unendlich ausgedehntes Gitter mit sinusförmigem Transmissionsverlauf und variabler Gitterkonstante, um unterschiedliche Raumfrequenzen abzudecken. Die Herstellung entsprechend präziser Nanostrukturen für den Einsatz im Spektralbereich weicher Röntgenstrahlung ist allerdings nahezu unmöglich. Meist wird daher auf Rechteckprofile statt des sinusförmigen Verlaufs zurückgegriffen. Des Weiteren führt die endliche Ausdehnung des 
Testobjekts zu weiteren Abweichungen, sodass die MTF mit direkten Messmethoden lediglich angenähert werden kann.

Alternativ wird die MTF indirekt berechnet, indem das Fourierspektrum des Transmissionsverlaufs eines beliebigen aber bekannten Objekts und der dazugehörigen Abbildung analysiert wird. Die MTF lässt sich z. B. aus der zweidimensionalen Fouriertransformation der Punktspreizfunktion PSF (engl. point spread function) berechnen :

$$
\operatorname{MTF}(\mathbf{u})=\mathcal{F}\{\operatorname{PSF}(\mathbf{x})\},
$$

wobei die PSF angibt, wie ein idealisiertes, punktförmiges Objekt im Mikroskop abgebildet wird.

Generell ist bei den beschriebenen Methoden zu beachten, dass nicht nur das Testobjekt und die abbildende Optik das Messergebnis beeinflussen, sondern auch der Detektor sowie das Beleuchtungssystem inklusive verwendeter Aperturen. Eine weitere Fehlerquelle stellt das Photonenrauschen dar, denn es überlagert die eigentlichen Bildinformationen. Die MTF wird daher speziell für hohe Raumfrequenzen verfälscht, da sie dort sehr kleine Werte annimmt. Das Rauschen lässt sich in guter Näherung durch eine Poisson-Verteilung beschreiben und es gilt [125]:

$$
\text { Photonenrauschen }=\sqrt{\text { Anzahl der Photonen }} .
$$

Das heißt zugleich, dass das Signal-Rausch-Verhältnis proportional zur Wurzel aus der Anzahl der Photonen zunimmt. Um Bilder hoher Qualität $\mathrm{zu}$ erhalten, werden etwa $N=1000$ Photonen pro Pixel benötigt $[21,125]$. Dies entspricht einem $S N R$ von $N / \sqrt{N} \approx 31,6$.

Um die MTF aus einzelnen Mikroskopbildern ermitteln zu können, sollte das Bild möglichst viele Raumfrequenzen enthalten. Als Teststruktur wird daher häufig ein Siemensstern (siehe Abb. 3.3a) verwendet, der ein kontinuierliches Raumfrequenzspektrum in radialer Richtung aufweist. Aufgrund der Rotationssymmetrie ist eine eindimensionale Beschreibung des Siemenssterns allerdings nicht möglich. Die indirekten

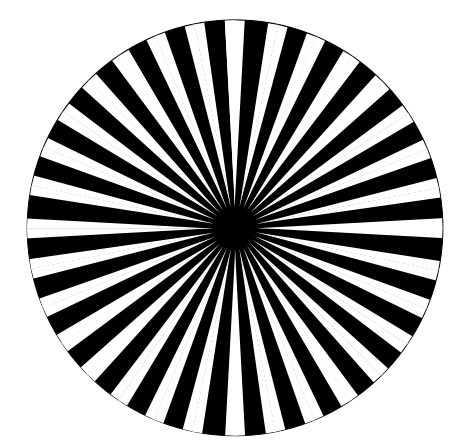

(a) Siemensstern

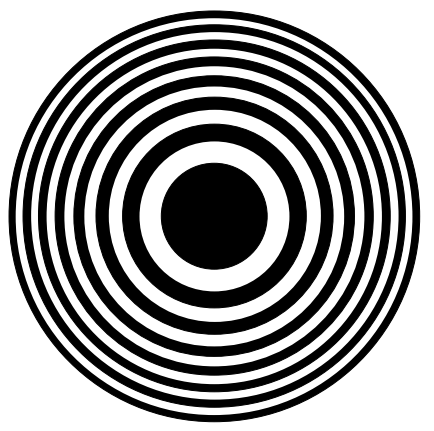

(b) Fresnel'sche Zonenplatte

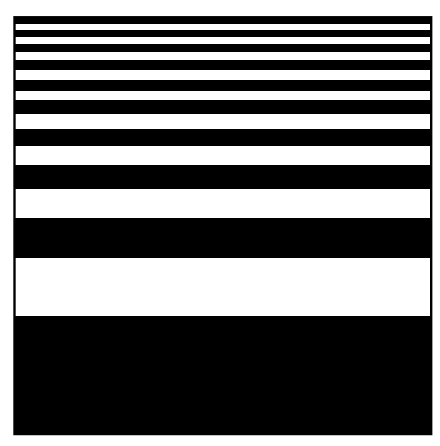

(c) Lineargitter

Abbildung 3.3: Mögliche Testobjekte zur Messung der MTF 
Verfahren zur Bestimmung der MTF sind somit eher ungeeignet. Dafür lässt sich die MTF für verschiedene Richtungen messen. Darüber hinaus können die Abbildungseigenschaften des Mikroskops unmittelbar ohne analytisches Verfahren beurteilt werden. Aus der Abbildung wird direkt sichtbar, bis zu welchem Radius die Strukturen aufgelöst sind. Zudem werden durch die Rotationssymmetrie richtungsabhängige Abbildungsfehler aufgedeckt. Des Weiteren eignen sich als mögliche Testobjekte zur Messung der MTF u. a. Kreisgitter mit radial variierender Gitterkonstante wie Fresnel'sche Zonenplatten oder lineare Gitter mit hyperbolischem Zonenbildungsgesetz (siehe Abb. 3.3b und 3.3c).

\subsubsection{OPTISCHE ELEMENTE}

Wie im vorherigen Abschnitt beschrieben, gibt es im Vergleich zum sichtbaren Spektralbereich große Unterschiede in der Wechselwirkung weicher Röntgenstrahlung mit Materie. Diese wirken sich unmittelbar auf die Art der Optiken aus, die in Röntgenmikroskopen zur Strahlfokussierung und zur Abbildung des Objekts verwendet werden können. Insbesondere der Einsatz klassischer Linsen ist nicht möglich. Aufgrund der starken Absorption weicher Röntgenstrahlung darf eine Linse lediglich wenige $100 \mathrm{~nm}$ dick sein, um eine Transmission von mindestens $10 \%$ zu gewährleisten. Da sich der Realteil des Brechungsindex aber nur minimal von Eins unterscheidet, ist mit dieser Linse keine signifikante Brechung und damit auch keine Fokussierung des Strahls möglich. Für harte Röntgenstrahlung $(E>10 \mathrm{keV})$ ist die Absorption hingegen deutlich geringer und an Synchrotronstrahlquellen werden zur Fokussierung daher mehrere bikonkave Linsen (engl. compound refractive lenses, CRL) eingesetzt [126, 127]. Für weiche Röntgenstrahlung können solche zusammengesetzten Linsen jedoch nicht verwendet werden. Üblicherweise wird deshalb auf reflektierende oder diffraktive Optiken zur Strahlmanipulation zurückgegriffen.

\section{REFLEKTIERENDE OPTIKEN}

Inwiefern eine ebene, elektromagnetische Welle an einer Grenzfläche zweier Materialien unterschiedlicher Brechungsindizes reflektiert wird, lässt sich quantitativ aus den Fresnel'schen Formeln berechnen. Bei senkrechtem Einfall zur Grenzfläche gilt für die Reflektivität [128]

$$
\mathrm{R}^{90^{\circ}}=\frac{\delta^{2}+\beta^{2}}{(2-\delta)^{2}+\beta^{2}} .
$$

Mit den in Abschnitt 3.I.I berechneten Werten für $\delta$ und $\beta$ ergibt sich für Kohlenstoff bei einer Wellenlänge von $2,88 \mathrm{~nm}$ somit ein sehr kleiner Wert von $\mathrm{R}^{90^{\circ}}=6,0 \times 10^{-6}$. Die Reflektivität anderer Materialien 
ist im Bereich weicher Röntgenstrahlung ähnlich gering. Auf diese Weise können Spiegel demnach nicht verwendet werden. Um die Reflektivität entsprechend zu erhöhen, gibt es zwei Möglichkeiten: Entweder wird der Spiegel unter streifendem Einfall genutzt (Totalreflexion) oder es werden sogenannte Multischichtspiegel eingesetzt. Letztere bestehen aus mehreren Schichten, die phasengerecht überlagert werden, um konstruktive Interferenz zwischen den reflektierten Teilstrahlen zu erhalten. Dazu werden Doppelschichten der Dicke d aus je einem Material hoher und niedriger Absorption auf ein Substrat aufgetragen. Entspricht der Weglängenunterschied unter dem gewünschten Einfallswinkel ${ }^{1} \theta$ einem Vielfachen $m$ der Wellenlänge $\lambda$, ist also die Bragg-Bedingung

$$
2 \mathrm{~d} \sin (\theta)=\mathrm{m} \lambda
$$

mit $m \in \mathbb{N}$ erfüllt, lassen sich theoretisch Reflektivitäten nahe 1 erreichen. Aufgrund der starken Absorption ist die Reflektivität aber tatsächlich geringer. Für extrem ultraviolette Strahlung bei einer Wellenlänge von $\lambda=13,5 \mathrm{~nm}$ sind mit Molybdän/Silizium-Spiegeln dennoch Reflektivitäten von bis zu 70\% möglich [129]. Die Winkelakzeptanz beträgt etwa $\theta= \pm 5^{\circ}$. Multischichtspiegel werden daher hauptsächlich zur Strahlführung und -fokussierung in der Halbleiterlithografie verwendet [130]. Die spektrale Bandbreite skaliert invers zur Schichtanzahl [131] und liegt für Spiegel mit 60 Schichten für senkrechten Einfall in der Größenordnung $\Delta \lambda \approx 0,5 \mathrm{~nm}$. Die Spiegel werden deshalb auch als Monochromatoren eingesetzt.

Wegen des großen Akzeptanzwinkels sind Multischichtspiegel auch als Kondensoroptiken für die Mikroskopie mit weicher Röntgenstrahlung von Interesse. Diese werden auf Basis von Scandium (Sc) sowie Titan (Ti) und Vanadium (V) entwickelt. Auf kleinen sphärischen Substraten können zwar Spiegel mit Reflektivitäten von bis zu 32\% hergestellt werden [132], aufgrund der geringen numerischen Apertur sind diese jedoch für den Einsatz in Mikroskopen ungeeignet. Die Reflektivität von Spiegeln mit ausreichend großen Durchmessern von etwa $60 \mathrm{~mm}$ liegt hingegen für nahezu senkrechten Einfall lediglich im Bereich von 0,5 bis 4,5\% [133, 134]. Die geringen Werte bei kurzen Wellenlängen sind auf die extrem hohen Anforderungen bei der Herstellung der einzelnen Schichten zurückzuführen. Die Schichtdicke für nahezu senkrechten Einfall entspricht gemäß der Bragg-Bedingung (3.12) nur etwa der halben Wellenlänge. Aufgrund von Diffusion zwischen den Schichten ist es aber schwierig, scharfe Übergänge an den Grenzflächen zwischen den Materialien zu erreichen. Durch den Einsatz zusätzlicher Barriereschichten (z. B. Borcarbid) lässt sich die Diffusion verringern und die Reflektivität der Spiegel erhöhen [135]. Eine weitere Schwierigkeit besteht darin, die

I Der Einfallswinkel wird in diesem Fall nicht zum Lot, sondern zur Oberfläche des Spiegels gemessen. 
Schichten gleichmäßig auf die gesamte Fläche des Substrats aufzutragen. Da sich der Einfallswinkel entlang der gekrümmten Oberfläche ändert, muss zudem die Schichtdicke entsprechend angepasst werden. Dazu wird eine Maske über dem rotierenden Substrat angebracht, während das Material aufgetragen wird [133]. Da die spektrale Breite der Spiegel aufgrund der vielen Schichten (bis zu 400) für weiche Röntgenstrahlung nur etwa $\Delta \lambda=0,01 \mathrm{~nm}$ beträgt, muss die Schichtdicke exakt an die Wellenlänge der Röntgenquelle angepasst sein. Andernfalls führen schon minimale Abweichungen zu einem deutlichen Reflektivitätsverlust. Insbesondere für Laborröntgenmikroskope mit laserinduzierten Plasmen ist dies problematisch, denn die Emissionswellenlänge des Plasmas ist durch einen elektronischen Übergang innerhalb des Plasmas gegeben und daher sehr schmalbandig.

Als Alternative zu Multischichtspiegeln werden Spiegel unter streifendem Einfall eingesetzt. Für Einfallswinkel (gemessen zur Oberfläche, nicht zum Lot) unterhalb eines kritischen Winkels $\theta_{c}$ wird eine elektromagnetische Welle beim Übergang von einem optisch dichten auf ein optisch dünneres Medium total reflektiert. Der kritische Winkel ergibt sich hierfür aus dem Snellius'schen Brechungsgesetz unter Vernachlässigung von Absorption $(\beta=0) \mathrm{zu}$

$$
\cos \left(\theta_{\mathrm{c}}\right)=1-\delta
$$

Wegen der geringen Werte von $\delta$ tritt Totalreflexion für weiche Röntgenstrahlung folglich nur unter sehr kleinen Winkeln auf, sodass für den kritischen Winkel mit der Näherung $\cos \left(\theta_{\mathcal{c}}\right) \approx 1-\theta_{\mathrm{c}}^{2} / 2$ gilt $[6,136]$ :

$$
\theta_{c} \approx \sqrt{2 \delta} \stackrel{G l .(3.4)}{=} \sqrt{\frac{n_{a} r_{e} \lambda^{2} f_{1}^{0}}{\pi}} \propto \lambda \sqrt{Z}
$$

Dabei wurde ausgenutzt, dass sich der Streufaktor $f_{1}^{0}$ durch die Ordnungszahl Z annähern lässt (vgl. Abb. 3.2). Elemente hoher Ordnungszahl z. B. Gold $(Z=79)$ eignen sich demnach besonders gut, um einen großen kritischen Winkel zu erreichen. Allerdings wurde bisher die Absorption weicher Röntgenstrahlung in Materie vernachlässigt.

Allgemein wird die Reflektivität einer elektromagnetischen Welle aus den Fresnel-Gleichungen berechnet (Herleitung siehe z. B. Ref. [6]):

$$
R=\frac{\theta^{2}-\sqrt{2} \theta \sqrt{\sqrt{\left(\theta^{2}-\theta_{c}^{2}\right)^{2}+4 \beta^{2}}+\left(\theta^{2}-\theta_{c}^{2}\right)}+\sqrt{\left(\theta^{2}-\theta_{c}^{2}\right)^{2}+4 \beta^{2}}}{\theta^{2}+\sqrt{2} \theta \sqrt{\sqrt{\left(\theta^{2}-\theta_{c}^{2}\right)^{2}+4 \beta^{2}}+\left(\theta^{2}-\theta_{c}^{2}\right)}+\sqrt{\left(\theta^{2}-\theta_{c}^{2}\right)^{2}+4 \beta^{2}}} .
$$

In Abbildung 3.4 ist die Reflektivität als Funktion des normierten Einfallswinkels $\theta / \theta_{c}$ für verschiedene Parameter $\beta / \delta$ aufgetragen: Je stärker 
das Material absorbiert, desto geringer ist dessen Reflektivität. Für weiche Röntgenstrahlung reflektieren daher mit Nickel $\left(Z=28, \theta_{c}^{\mathrm{Ni}}=6,5^{\circ}\right)$ beschichtete Spiegel besser als Goldspiegel, obwohl die Ordnungszahl von Gold deutlich höher ist. Bei einer Wellenlänge von $\lambda=2,88 \mathrm{~nm}$ beträgt das Verhältnis zwischen Absorption und Brechung für Nickel aber lediglich $\beta / \delta=0,28$, sodass die Reflektivität deutlich geringer vom Idealfall der Totalreflexion abweicht als für Gold $(\beta / \delta=1,1)$ [120].

Konkavspiegel unter streifendem Einfall eignen sich also zur Fokussierung weicher Röntgenstrahlung und werden in der Mikroskopie häufig als Kondensoroptiken eingesetzt. Verwendet werden sphärisch, elliptisch, parabolisch oder hyperbolisch geformte Spiegel. Diese Einspiegelsysteme weisen jedoch Aberrationen wie Astigmatismus oder Koma auf [137]. Weit verbreitet sind zur Fokussierung daher Anordnungen aus mehreren Spiegeln nach Kirkpatrick-Baez [138], Wolter [117] und Montel [139], mit denen Abbildungsfehler verringert werden können. Nachteilig ist die aufwendige Justage der Mehrfachspiegelsysteme.

Unabhängig von der Spiegelgeometrie wird immer ein Teil der Strahlung an den Spiegeloberflächen gestreut. Je nach Herstellungsprozess beträgt die Oberflächenrauheit 0,5 bis $2 \mathrm{~nm}$ (mittlere quadratische $\mathrm{Ab}$ weichung) [140]. Dies führt zu einer verminderten Reflektivität des Spiegels. Außerdem vergrößert sich der Fokusdurchmesser, weil das gestreute Licht rings um den Fokuspunkt seitliche Ausläufer entstehen lässt.

\section{DIFFRAKTIVE OPTIKEN}

Wie eben beschrieben, eignen sich für die Fokussierung weicher Röntgenstrahlung speziell Spiegel unter streifendem Einfall. Ihr Auflösungsvermögen ist aufgrund von Abbildungsfehlern aber begrenzt. Um eine

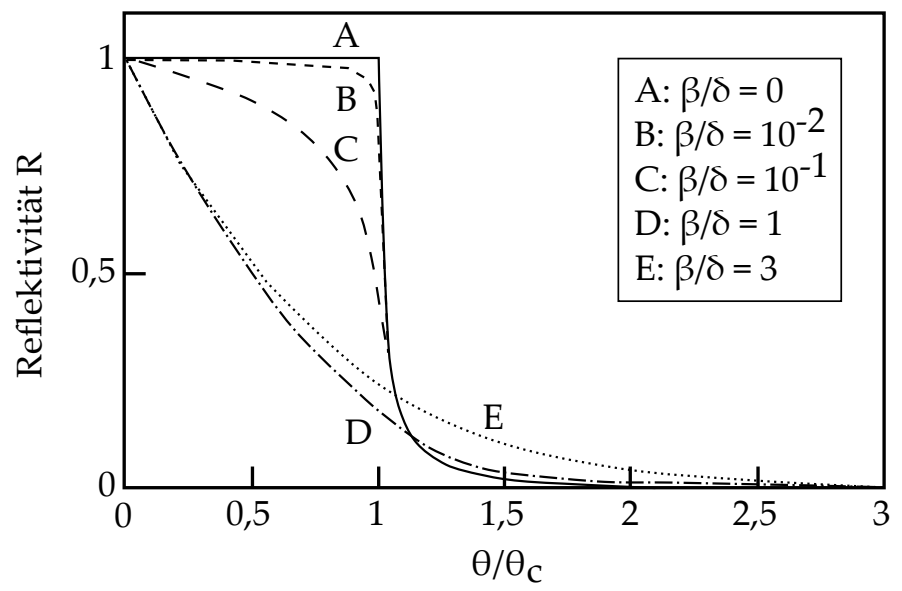

Abbildung 3.4: Reflektivität an einer Grenzschicht zwischen Vakuum und Materie als Funktion des normierten Einfallswinkels $\theta / \theta_{c}$ für verschiedene Parameter $\beta / \delta$ (nach Ref. [6]). Der Verlauf der Reflektivität gilt für senkrecht und parallel polarisierte Röntgenstrahlung. 
räumliche Auflösung nahe des Beugungslimits zu erreichen, werden daher diffraktive Optiken verwendet. Zum Beispiel werden Beugungsgitter als Monochromatoren an Synchrotronstrahlquellen und als dispersive Elemente in Spektrometern (siehe Abs. 4.4) eingesetzt. Zur hochauflösenden Abbildung werden hingegen Fresnel'sche Zonenplatten genutzt, deren Funktionsweise im Folgenden beschrieben wird. Die Darstellung orientiert sich an den Referenzen [6] und [119].

Fresnel'sche Zonenplatten sind Kreisgitter aus abwechselnd absorbierenden und transmittierenden Ringen (siehe Abb. 3.5), deren Breite nach außen abnimmt. Dadurch verringert sich die Gitterperiode und die Beu-

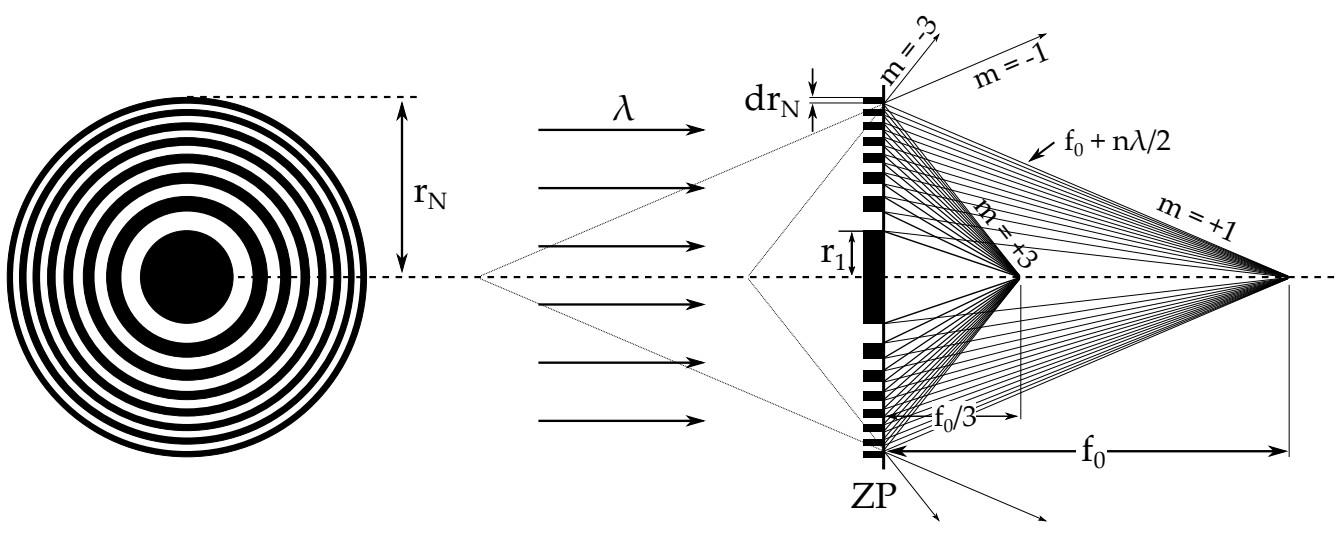

Abbildung 3.5: Schematische Darstellung einer Fresnel'schen Zonenplatte (nach Ref. [6]). Die Parameter sind im Text erklärt.

gungswinkel der transmittierten Teilstrahlen werden in radialer Richtung größer. Ändern sich die Radien der einzelnen Ringe im richtigen Verhältnis zueinander, ist es demnach möglich, dass die Teilstrahlen konstruktiv in einem gemeinsamen Fokus interferieren. Die wichtigsten Größen der Zonenplatte wie Brennweite $f_{0}$, numerische Apertur NA, Auflösungsvermögen $\delta^{\text {Rayl. }}$ und Schärfentiefe $\Delta z$ werden dabei durch die Wellenlänge $\lambda$ der einfallenden Strahlung, die Anzahl der Zonen $N$ und die Breite $d r_{N}$ der äußersten Zone charakterisiert.

Wenn der Weglängenunterschied zwischen benachbarten transmittierenden Zonen der Wellenlänge $\lambda$ entspricht, interferieren die Teilstrahlen konstruktiv im Fokus der ersten Beugungsordnung. Die optische Weglänge $\sqrt{r_{n}^{2}+f_{0}^{2}}-n \lambda / 2$ ist demnach gleich der Brennweite $f_{0}$. Eine Reihenentwicklung der Wurzel bis zu Termen $r_{n}^{4}$ ergibt für den Radius der n-ten Zone

$$
r_{n}^{2}=n \lambda f_{0}+\frac{n^{2} \lambda^{2}}{4}
$$

Im Bereich weicher Röntgenstrahlung ist die numerische Apertur (siehe Tab. 3.1) einer Zonenplatte meist sehr viel kleiner als Eins. Der Term $n^{2} \lambda^{2} / 4$ lässt sich somit vernachlässigen, weil $f_{0} \gg n \lambda / 2$ gilt. Für höhere Wellenlängen sollte der Term allerdings berücksichtigt werden, um 
sphärische Aberrationen zu korrigieren. Für kleine numerische Aperturen hingegen vereinfacht sich Gleichung (3.16) zu

$$
r_{\mathrm{n}} \approx \sqrt{n \lambda f_{0}}
$$

Die Teilstrahlen interferieren demnach konstruktiv, wenn die Radien benachbarter Ringe mit $\sqrt{n}$ größer werden. Aus Gleichung (3.17) lassen sich die charakteristischen Parameter einer Zonenplatte ableiten (siehe Tab. 3.1, Herleitung siehe Ref. [6]), aus denen einige wichtige Eigenschaften einer Zonenplatte folgen:

Tabelle 3.1: Charakteristische Größen Fresnel'scher Zonenplatten für die hochauflösende Abbildung bei $\lambda=2,88 \mathrm{~nm}$ in der ersten Beugungsordnung

\begin{tabular}{|c|c|c|}
\hline Parameter & Formel & typische Werte \\
\hline Breite äußerster Ring & $d r_{N}=\frac{\lambda f_{O}}{2 r_{N}}$ & $25-80 \mathrm{~nm}$ \\
\hline Durchmesser & $\mathrm{D}=2 \mathrm{r}_{\mathrm{N}}=4 \mathrm{Nd} \mathrm{r}_{\mathrm{N}}$ & $80-200 \mu \mathrm{m}$ \\
\hline Brennweite & $f_{0}=\frac{4 N\left(d r_{N}\right)^{2}}{\lambda}$ & $0,7-5,5 \mathrm{~mm}$ \\
\hline numerische Apertur & $N A=\frac{r_{N}}{f_{0}}=\frac{\lambda}{2 d r_{N}}$ & $0,058-0,018$ \\
\hline Auflösungsvermögen & $\delta^{\text {Rayl. }}=1,22 \mathrm{dr}_{\mathrm{N}}=\frac{0,61 \lambda}{\mathrm{NA}}$ & $30-100 \mathrm{~nm}$ \\
\hline Schärfentiefe & $\Delta z= \pm \frac{\lambda}{2 N A^{2}}= \pm \frac{2\left(\mathrm{dr}_{\mathrm{N}}\right)^{2}}{\lambda}$ & $0,45-4,5 \mu \mathrm{m}$ \\
\hline
\end{tabular}

1. Die Brennweite ist umgekehrt proportional zur Wellenlänge. Die chromatische Aberration ist demnach sehr viel größer als für eine „klassische“ Linse.

2. Das Auflösungsvermögen ist hingegen unabhängig von der Wellenlänge und gemäß Rayleigh-Kriterium nur durch die Breite der äußersten Zone bestimmt.

3. Die Fläche $\pi\left(r_{n}^{2}-r_{n-1}^{2}\right)=\pi \lambda f_{0}$ zweier benachbarter Ringe ist konstant. Alle Zonen tragen daher gleichermaßen zur Intensität im Fokus bei.

4. Die Schärfentiefe $\Delta z$ skaliert invers zum Quadrat der numerischen Apertur. Sie gibt den Bereich um den Brennpunkt an, in dem die Intensität nur um maximal 20\% abnimmt [6]. Für Zonenplatten mit großer Apertur, die potenziell die höchste räumliche Auflösung erreichen, ist die Schärfentiefe dementsprechend sehr gering. Das Objekt muss daher exakt im Brennpunkt positioniert werden können. Zudem begrenzt die Schärfentiefe den Bereich eines ausgedehnten dreidimensionalen Objekts, der scharf abgebildet wird. 
5. Aufgrund der Wellenlängenabhängigkeit der Brennweite muss die Quelle hinreichend monochromatisch sein, um chromatische Aberrationen in der Abbildung des Objekts zu verhindern. Aus der Bedingung, dass die Fokuslängenvariation nicht größer als die Schärfentiefe sein darf, folgt für die spektrale Bandbreite: $\Delta \lambda / \lambda \leqslant 1 / N$. Bei typischerweise einigen hundert Zonen muss die relative Bandbreite der Quelle daher deutlich kleiner als $1 \%$ sein.

Fresnel'sche Zonenplatten werden aufgrund ihrer Eigenschaften in der Röntgenmikroskopie als Kondensoroptiken zur Strahlfokussierung und als Objektive zur hochauflösenden Abbildung verwendet. Da die Zonenplatte wie eine Linse wirkt, gilt das Abbildungsgesetz

$$
\frac{1}{f_{0}}=\frac{1}{g}+\frac{1}{b}
$$

Ein Objekt im Abstand $g$ zur Zonenplatte wird demnach in die Bildebene im Abstand $b$ abgebildet. Die Vergrößerung beträgt $M=b / g$ und liegt typischerweise im Bereich von 100 bis 1000.

Bisher wurde jedoch nur die erste Beugungsordnung der Zonenplatte betrachtet. Wie bei jedem Gitter treten aber immer ganzzahlige Ordnungen $m \in \mathbb{Z}$ auf. Neben der unfokussierten o. Ordnung gibt es demnach eine Reihe zusammenlaufender $(+m)$ und divergenter $(-m)$ Strahlen mit Brennweite $f_{0} / m$ bzw. $-f_{0} / m$ (siehe Abb. 3.5). Die numerische Apertur ist in der m-ten Ordnung um den Faktor $m$ größer, die Auflösung entsprechend m-fach besser. Da alle Ringe die gleiche Fläche haben, löschen sich die geraden Ordnungen im Fokus allerdings aus.

Labormikroskope basieren auf kompakten Röntgenquellen, deren Brillanz deutlich kleiner ist als die von Synchrotronen. Um dennoch ein Objekt bei hoher Auflösung mit gutem Kontrast abbilden zu können, muss die Zonenplatte einen möglichst hohen Beugungswirkungsgrad $\eta$ aufweisen. Dieser gibt das Verhältnis der Intensität $\mathrm{I}_{m}$ in der m-ten Beugungsordnung zur Gesamtintensität I an. Für eine dünne, symmetrische Zonenplatte (Aspektverhältnis von Zonentiefe zu Zonenbreite $<10: 1$ ) und $m \neq 0$ gilt $[141]$

$$
\eta_{m}=\frac{I_{m}}{I}=\frac{1}{(m \pi)^{2}}\left[1+e^{-4 \pi \beta t / \lambda}-2 e^{-2 \pi \beta t / \lambda} \cos (2 \pi \delta t / \lambda)\right],
$$

wobei $\mathrm{t}$ die Zonentiefe der Zonenplatte bezeichnet. Der Anteil der 0. Beugungsordnung $(m=0)$ und absorbierten Intensität $I_{a b s}$ wird nach

$$
\begin{aligned}
\eta_{0} & =\frac{I_{0}}{I}=\frac{1}{4}\left[1+e^{-4 \pi \beta t / \lambda}+2 e^{-2 \pi \beta t / \lambda} \cos (2 \pi \delta t / \lambda)\right] \text { und } \\
\eta_{a b s} & =\frac{I_{a b s}}{I}=\frac{1}{2}\left(1+e^{-4 \pi \beta t / \lambda}\right)
\end{aligned}
$$

berechnet. Hinsichtlich ihrer Effizienz werden drei Arten von Zonenplatte unterschieden: Amplituden- bzw. Absorptionszonenplatten (Fresnel), 
phasenschiebende Zonenplatten (Rayleigh-Wood) und mehrstufige phasenschiebende Zonenplatten (Multilevel).

Bei Fresnel'schen Zonenplatten $(\beta \rightarrow \infty)$ wird die Hälfte der einfallenden Strahlung in den opaken Zonen absorbiert. Weitere $25 \%$ werden nicht gebeugt (0. Beugungsordnung). Die restliche Intensität verteilt sich auf die ungeraden Beugungsordnungen, wobei der Wirkungsgrad quadratisch mit $\mathrm{m}$ abnimmt:

$$
\eta_{\mathrm{FZP}}=\frac{1}{(\pi \mathrm{m})^{2}} \text {. }
$$

In die erste Beugungsordnung werden etwa 10\% der transmittierten Strahlung fokussiert. Die für eine vollständige Absorption (>99\%) erforderliche Zonentiefe $t$ lässt sich anhand des Lambert-Beer'schen Gesetzes (siehe Gl. (3.2)) bestimmen. Sie ist umso geringer, je stärker das Material der Fresnel'schen Zonenplatte die weiche Röntgenstrahlung absorbiert.

Bei einer phasenschiebenden Zonenplatte wird durch die Wahl eines transparenten Zonenmaterials in den Beugungsordnungen eine deutlich höhere Effizienz erreicht. Für eine bestimmte Zonentiefe erfährt die durch die transparenten Zonen transmittierte Strahlung einen Phasenschub von $\varphi=2 \pi \delta \mathrm{t} / \lambda=\pi$. Im Idealfall $(\beta \rightarrow 0)$ wird demnach keine Strahlung absorbiert und zudem die 0 . Beugungsordnung vollständig unterdrückt. Damit ergibt sich in allen Beugungsordnungen eine Vervierfachung der Intensität. Insbesondere für weiche Röntgenstrahlung kann die Absorption in den Zonen aber nicht vernachlässigt werden. Dadurch wird die Beugungseffizienz deutlich verringert. In Abbildung 3.6 sind für einige Materialien, die zur Herstellung von Zonenplatten verwendet werden, die theoretisch erreichbaren Wirkungsgrade für eine Photonenenergie von $E=430 \mathrm{eV}$ aufgetragen. Der Phaseneffekt in Abhängigkeit der Zonentiefe ist für alle gezeigten Materialien deutlich sichtbar. Für Beryllium (Be) ist das Verhältnis $\xi=\beta / \delta$ mit 0,15 verglichen mit Nickel $(\mathrm{Ni}, \xi=0,28)$, Siliziumnitrid $\left(\mathrm{Si}_{3} \mathrm{~N}_{4}, \xi=0,52\right)$ und Wolfram $(\mathrm{W}, \xi=0,88)$ am geringsten. Daher ergibt sich für Beryllium die höchste Beugungseffizienz. Allerdings beträgt die Zonentiefe dabei $670 \mathrm{~nm}$, die durch lithografische Herstellungsprozesse kaum realisierbar ist. Mit zunehmendem $\xi$ nimmt die Beugungseffizienz ab, da die Absorption innerhalb der Zonen dominiert. Aufgrund der größeren Phasenmodulation sind aber gleichzeitig kleinere Zonentiefen nötig. Für die Herstellung hochauflösender Zonenplatten mit möglichst geringer äuBerster Zonenbreite sind diese Materialien deshalb vorzuziehen.

Mehrstufige phasenschiebende Zonenplatten erreichen theoretisch eine Effizienz von fast $100 \%$ in der ersten Beugungsordnung. Bei diesen Multilevel-Zonenplatten ändert sich der Phasenschub zwischen offener und transmittierender Zone nicht abrupt von 0 auf $\pi$, sondern kontinuierlich (Kinoform). Dazu werden die teiltransparenten Zonen in Strahlrichtung mit beliebig vielen stufenförmigen Unterzonen (Phasenlevel) L 


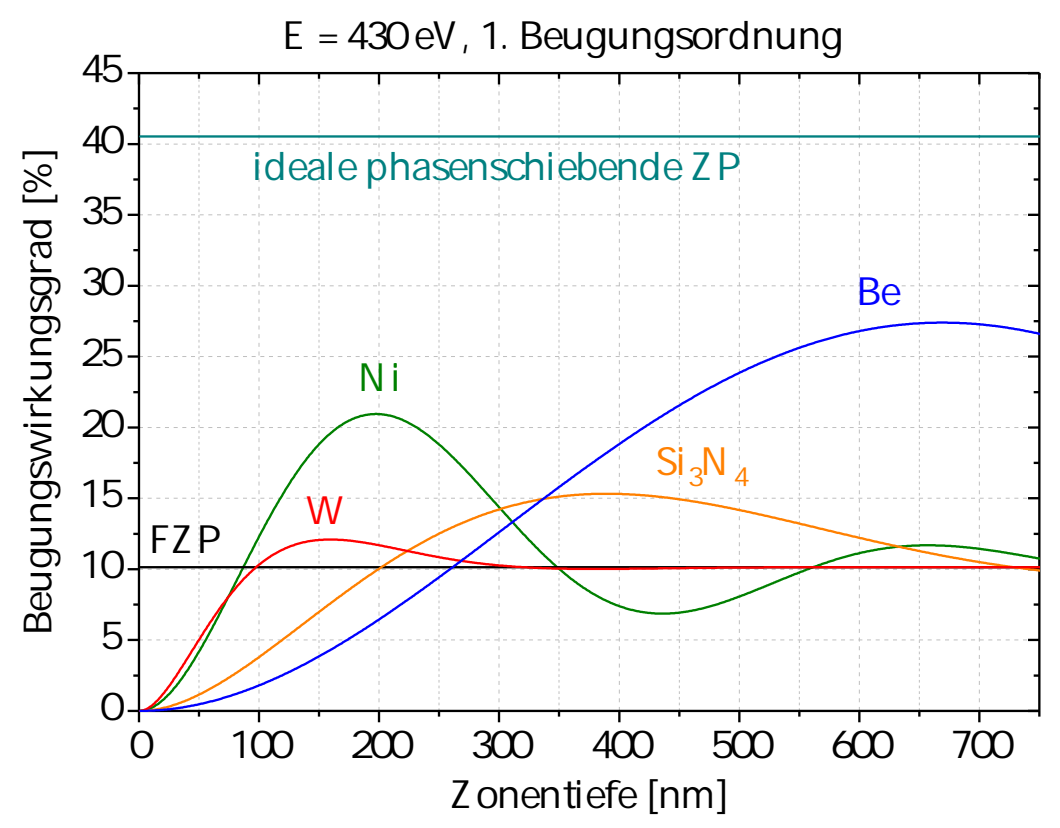

Abbildung 3.6: Berechnete Wirkungsgrade $\eta_{1}$ phasenschiebender Zonenplatten aus verschiedenen Materialien sowie Effizienz einer Fresnel'sche Zonenplatte $(\beta \rightarrow \infty)$ und einer idealen phasenschiebenden Zonenplatte $(\beta \rightarrow 0)$. Die Werte für $\delta$ und $\beta$ sind Referenz [120] entnommen.

über ein parabolisches Tiefenprofil angenähert. Die äußerste Breite einer mehrstufigen Zonenplatte ist größer als bei einer binären Zonenplatte (z. B. Faktor 2 bei einer quaternären $(L=4)$ Zonenplatte). Dadurch ist auch das Auflösungsvermögen verringert. Deshalb muss je nach Anwendung die passende Zonenplatte ausgewählt werden, um entweder vom höheren Beugungswirkungsgrad mehrstufiger oder von der besseren Auflösung Fresnel'scher Zonenplatten zu profitieren.

Zonenplatten werden konventionell in einem Verfahren aus Elektronenstrahl-Lithografie und reaktivem Ionenätzen hergestellt [142, 143]. Die äußerste Zone sollte dabei eine möglichst geringe Breite aufweisen, um eine hohe Auflösung zu ermöglichen. Dies ist jedoch nur bedingt möglich, da der Elektronenstrahl nicht beliebig klein fokussiert werden kann. Zudem verhindert die Entstehung von Sekundärelektronen im Polymer die Herstellung hoher Zonendichten. Aufgrund der optischen Eigenschaften typischer Zonenplattenmaterialien sind für einen Phasenschub von $\pi$ und damit einer optimalen Effizienz außerdem Zonentiefen von mindestens $100 \mathrm{~nm}$ erforderlich. In Verbindung mit den kleinen äußeren Strukturen der Zonenplatte resultieren daraus hohe Aspektverhältnisse, die lithografisch eine große Herausforderung darstellen. Mit fortgeschrittenen Techniken lassen sich diese Schwierigkeiten überwinden. So werden die Lithografie- und Ätzprozesse mehrfach wiederholt. Werner et al. demonstrierten auf diese Weise ein Aspektverhältnis von 20 : 1 für eine Nickel-Zonenplatte [144]. Alternativ können zwei oder 
mehr Zonenplatten hintereinander gestapelt werden, um ein höheres Aspektverhältnis zu erreichen [145, 146]. Ist deren Periode unterschiedlich, entstehen so außerdem mehrstufige Zonenplatten [147].

Für die hochauflösende Abbildung im Bereich weicher Röntgenstrahlung beträgt die kleinste, bisher gefertigte äußerste Zonenbreite $12 \mathrm{~nm}$ [123]. Dazu wurden zwei komplementäre Zonenplatten mit einer Positioniergenauigkeit von $2 \mathrm{~nm}$ ineinander verschachtelt. Eine andere Möglichkeit die äußerste Zonenbreite zu verringern, besteht in der nachträglichen Deposition von Zonenplattenmaterial auf bereits geätzten Strukturen [148]. Mit dieser Art von Zonenplatte wurde bei einer Energie von 1,2 keV eine räumliche Auflösung von $9 \mathrm{~nm}$ erreicht [149]. Mit den genannten Verfahren wurden bisher allerdings nur einzelne Prototypen hergestellt. Bei kommerziell erhältlichen Zonenplatten ist die Breite der äußersten Zonen hingegen auf ungefähr $25 \mathrm{~nm}$ und das Aspektverhältnis auf etwa 10:1 beschränkt. Die Zonenplatten, die im Rahmen dieser Arbeit verwendet wurden, werden in Abschnitt 3.3.1 beschrieben.

\subsubsection{KOH ÄRENZ}

Je nach Quelle weist Röntgenstrahlung unterschiedliche Eigenschaften auf, die abhängig vom Abbildungsverfahren das Auflösungsvermögen und den Kontrast eines Bildes beeinflussen können. Ein wichtiges Merkmal von Strahlung ist die Kohärenz. Sie charakterisiert ganz generell die Korrelation zwischen zwei Größen eines Wellenfeldes. Ein Phänomen, um diese Korrelation zu veranschaulichen, ist Interferenz. Im klassischen Sinne kennzeichnet der Begriff Kohärenz daher die Fähigkeit von Wellen, räumlich und zeitlich stabile Interferenzmuster zu bilden. Im Folgenden werden die wesentlichen Konzepte der Kohärenz erläutert. Die Darstellung orientiert sich dabei an Referenz [150].

Für ein quasi-stationäres Wellenfeld $E(r, t)$ mit Periodendauer $T$ wird die Kohärenz mathematisch durch die komplexe wechselseitige Kohärenzfunktion (engl. mutual coherence function)

$$
\begin{aligned}
\Gamma\left(\mathbf{r}_{1}, \mathbf{r}_{2}, \mathrm{t}_{1}, \mathrm{t}_{2}\right) & =\Gamma_{12}\left(\mathrm{t}_{1}, \mathrm{t}_{2}\right)=\Gamma_{12}(\tau)=\left\langle\mathrm{E}\left(\mathbf{r}_{1}, \mathrm{t}+\tau\right) \mathrm{E}^{*}\left(\mathbf{r}_{2}, \mathrm{t}\right)\right\rangle \\
& =\lim _{\mathrm{T} \rightarrow \infty} \frac{1}{\mathrm{~T}} \int_{-\mathrm{T} / 2}^{\mathrm{T} / 2} \mathrm{E}\left(\mathbf{r}_{1}, \mathrm{t}+\tau\right) \mathrm{E}^{*}\left(\mathbf{r}_{2}, \mathrm{t}\right) \mathrm{dt}
\end{aligned}
$$

beschrieben. Diese hängt neben der Zeitverschiebung $\tau=t_{2}-t_{1}$ von den beiden Raumpunkten $\mathbf{r}_{1}$ und $\mathbf{r}_{2}$ ab. Die spitzen Klammern bezeichnen dabei den zeitlichen Mittelwert und $E^{*}$ kennzeichnet das komplex Konjugierte zu E. Der komplexe Kohärenzgrad entspricht damit der normierten Kohärenzfunktion:

$$
\gamma_{12}(\tau)=\frac{\Gamma_{12}(\tau)}{\sqrt{\Gamma_{11}(0) \Gamma_{22}(0)}},
$$


wobei $\Gamma_{11}(0)$ und $\Gamma_{22}(0)$ die Intensitäten des Wellenfeldes am Ort $\mathbf{r}_{1}$ bzw. $\mathbf{r}_{2}$ bezeichnen. Je nach Betrag des Kohärenzgrades werden folgende drei Fälle unterschieden:

$$
\begin{aligned}
\left|\gamma_{12}\right|=1 & \text { vollständige Kohärenz, } \\
0<\left|\gamma_{12}\right|<1 & \text { partielle Kohärenz und } \\
\left|\gamma_{12}\right|=0 & \text { vollständige Inkohärenz. }
\end{aligned}
$$

Wie eingangs beschrieben, ist Kohärenz eng mit der Interferenzfähigkeit von Strahlung verbunden. Aus dem Betrag des Kohärenzgrades lässt sich daher der Kontrast $\mathrm{K}$ des Interferenzmusters berechnen:

$$
\mathrm{K}=\frac{\mathrm{I}_{\max }-\mathrm{I}_{\min }}{\mathrm{I}_{\max }+\mathrm{I}_{\min }}=\left|\gamma_{12}(\tau)\right|
$$

wobei hier gleiche Intensitäten der interferierenden Wellen angenommen wurden. In der Praxis wird dieser Zusammenhang meist umgekehrt genutzt, um aus dem gemessenen Interferenzmuster den Kohärenzgrad zu ermitteln.

Aus dieser generellen Beschreibung des Kohärenzbegriffs lassen sich zwei Grenzfälle ableiten: zeitliche $\left(\mathbf{r}_{1}=\mathbf{r}_{2}\right)$ und räumliche $(\tau=0)$ Kohärenz, die beide nachfolgend beschrieben werden.

\section{ZEITLICHE KOHÄRENZ}

Die zeitliche Kohärenz ergibt sich aus dem Vergleich einer Welle mit sich selbst zu verschiedenen Zeitpunkten. Die Kohärenzeigenschaften werden dabei durch die Kohärenzzeit $\tau_{\mathfrak{c}}$ charakterisiert. Diese ist als die Zeitverschiebung $\tau$ definiert, bei der die Kontrastfunktion auf den Wert $1 /$ e abgefallen ist. Die Welle legt in dieser Zeit die Strecke

$$
\xi_{t}=c_{0} \tau_{c}
$$

zurück ( $c_{0}$ : Lichtgeschwindigkeit), die deshalb als longitudinale Kohärenzlänge bezeichnet wird. Sie beschreibt die Kohärenzeigenschaften des Strahlungsfeldes in Ausbreitungsrichtung.

Gemäß Wiener-Chintschin-Theorem ist die Kohärenzzeit über eine Fouriertransformation mit der Frequenzbreite $\Delta v$ verknüpft [151, 152]:

$$
\xi_{\mathrm{t}}=\mathrm{c}_{0} \tau_{\mathrm{c}} \propto \frac{\mathrm{c}_{0}}{\Delta v}=\frac{\lambda^{2}}{\Delta \lambda}
$$

Für eine Quelle der Wellenlänge $\lambda$ nimmt die Kohärenzlänge folglich mit zunehmender Bandbreite $\Delta \lambda$ ab. Experimentell lässt sich die zeitliche Kohärenz z. B. mit einem Michelson-Interferometer bestimmen [153]. 


\section{RÄUMLICHE KOH ÄHERENZ}

Um Lichtquellen hinsichtlich ihrer räumlichen Kohärenz zu charakterisieren, wird die Interferenz von Wellen unterschiedlicher Ausgangspunkte betrachtet. Dazu eignet sich u. a. der Young'sche Doppelspaltversuch [154]. Dabei werden zwei Lochblenden, die sich im Abstand a zueinander befinden, von einer inkohärenten Quelle der Ausdehnung $\mathrm{D}$ mit Strahlung der Wellenlänge $\lambda$ beleuchtet. Der Abstand zwischen Quelle und Apertur beträgt $\mathrm{R}_{\mathrm{Q}}$. Für die Entstehung eines Interferenzmusters muss dann

$$
a \leqslant \frac{R_{Q} \lambda}{2 D}
$$

gelten (zur Herleitung siehe [150]). Die Fähigkeit zur Interferenz geht demnach mit zunehmendem Quelldurchmesser D verloren, kann aber wiederum durch einen größeren Abstand der Lochblenden zur Quelle erhöht werden. Um z. B. mit weicher Röntgenstrahlung bei $\lambda=2,88 \mathrm{~nm}$ eine transversale Kohärenzlänge von $\mathrm{a}=10 \mu \mathrm{m} \mathrm{zu}$ erreichen, ist für einen Quelldurchmesser von $\mathrm{D}=350 \mu \mathrm{m}$ (typische Ausdehnung des Plasmas in y-Richtung, siehe Abs. 2.3.4) ein Abstand zur Quelle von mindestens $R_{Q}=2,4 \mathrm{~m}$ nötig.

\section{VAN-CITTERT-ZERNIKE-THEOREM}

Die Messung der transversalen Kohärenzlänge aus dem Young'schen Doppelspaltversuch beruht auf einigen Näherungen. So wird z. B. die Intensitätsverteilung der Quelle vernachlässigt. Wesentlich genauer kann der räumliche Kohärenzgrad einer ausgedehnten Quelle mit dem VanCittert-Zernike-Theorem bestimmt werden. Unter der Annahme einer kleinen Quellausdehnung und großer Beobachtungsabstände entspricht der Kohärenzgrad der Fouriertransformierten der Quellintensität [6]:

$$
\gamma_{12}=\frac{e^{-i \varphi} \iint I(u, v) e^{i k\left(u \vartheta_{x}+v \vartheta_{y}\right)} d u d v}{\iint I(u, v) d u d v} .
$$

Dabei sind $u$ und $v$ die räumlichen Koordinaten in der Quellebene, $\vartheta_{x}$ und $\vartheta_{y}$ die Winkel zum betrachteten Punkt $\mathrm{P}(x, y)$ im Abstand $z$ in der Detektorebene und $\varphi=k\left(x^{2}+y^{2}\right) /(2 z)$ der Phasenterm mit Wellenzahl $k=2 \pi / \lambda$.

\subsubsection{KONZEPTE DER RÖNTGENMIKROSKOPIE}

Um ein Objekt mit weicher Röntgenstrahlung abzubilden, gibt es wie mit sichtbarem Licht verschiedene Möglichkeiten. So werden Transmissions- und Rasterröntgenmikroskope eingesetzt oder auch "linsenlose" Verfahren verwendet. Die wesentlichen Merkmale dieser unterschiedlichen Abbildungsmethoden werden im Folgenden erläutert. 


\section{TRANSMISSIONSMIKROSKOPIE}

Transmissionsröntgenmikroskope (engl. transmission x-ray microscope, TXM) sind analog aufgebaut zu Durchlichtmikroskopen im sichtbaren Spektralbereich (siehe Abb. 3.7): Das Objekt wird von einem Kondensor vollständig ausgeleuchtet und mit einem Objektiv vergrößert auf einen ortsauflösenden Detektor abgebildet. In synchrotronbasierten Mi-
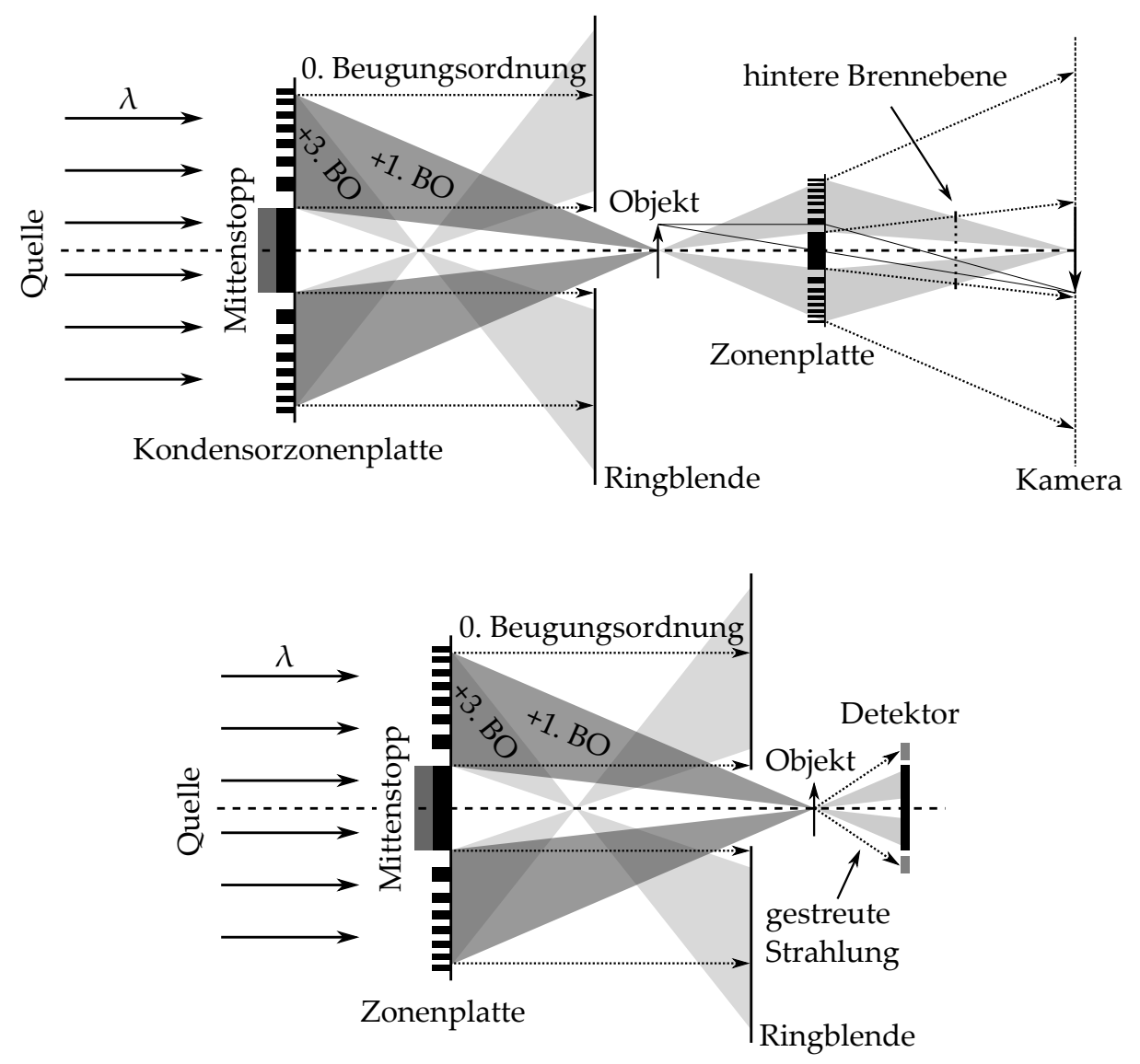

Abbildung 3.7: Schematischer Aufbau eines Transmissions- (oben) und Rasterröntgenmikroskops (unten, nach Ref. [6]).

kroskopen werden als Kondensoroptiken meist Zonenplatten verwendet. Schmahl et al. konnten so erstmals 1976 biologische Proben bei einer Wellenlänge von $\lambda=4,6 \mathrm{~nm}$ abbilden [12, 155]. Alternativ kommen insbesondere in Laborröntgenmikroskopen auch Multischichtspiegel [21] oder Spiegel unter streifendem Einfall [156] zum Einsatz. Ein Mittenstopp vor dem Kondensor verhindert, dass Röntgenstrahlung direkt auf den Detektor gelangt und dadurch den Kontrast der Abbildung verringert. Zudem werden mit einer Ringblende die höheren Beugungsordnungen der Zonenplatte geblockt. Diese würden sonst ebenso zu einem unerwünschten Untergrundsignal in der Bildebene führen. Die Abbildung des Objekts erfolgt im Absorptionskontrast mit einer einzelnen Zonenplatte, deren äußerste Zonenbreite das Auflösungsvermögen des 
Transmissionsmikroskops bestimmt. Um die höchste Auflösung zu erreichen, muss die numerische Apertur der Zonenplatte an die des Kondensors angepasst sein [157]. Als Detektoren werden in aller Regel Kameras mit CCD-Sensoren (engl. charge-coupled device) eingesetzt. Ein großer Vorteil von Transmissionsmikroskopen ist, dass für eine Abbildung im Absorptionskontrast die Quelle räumlich inkohärent sein kann. Bei partiell kohärenter Strahlung kann das Objekt mit Phasenkontrast abgebildet werden, wenn in die hintere Brennebene der Mikro-Zonenplatte zusätzlich eine ringförmige Phasenplatte gestellt wird [158].

Aufgrund der starken Absorption weicher Röntgenstrahlung in Materie dürfen die Proben maximal einige Mikrometer dick sein, um eine hinreichend hohe Transmission zu gewährleisten. Daher werden vereinzelt Mikroskope betrieben, in denen das Objekt in Reflexion untersucht wird [159, 160]. Wegen der geringen Eindringtiefe der Strahlung in Materie ist diese Methode besonders oberflächensensitiv.

\section{RASTERMIKROSKOPIE}

In Rasterröntgenmikroskopen (engl. scanning x-ray microscope, SXM) wird das Objekt nicht als Ganzes, sondern Punkt für Punkt abgebildet. Dazu wird die Röntgenstrahlung mit einer Zonenplatte auf das Objekt fokussiert (siehe Abb. 3.7). Ein Mittenstopp verhindert die direkte Beleuchtung des Objekts, und eine Ringblende blockt alle unerwünschten Beugungsordnungen. Während das Objekt durch den Fokus bewegt wird, wird die transmittierte Strahlung zeitaufgelöst von einem Detektor mit hoher Sensitivität aufgenommen. Typischerweise werden dazu mit Gas gefüllte Zählrohre, Fotodioden oder Phosphorschirme in Kombination mit einem Fotomultiplier verwendet [119]. Lässt sich das $\mathrm{Ob}-$ jekt hinreichend exakt im Fokus verfahren, ist das Auflösungsvermögen durch die Fokusgröße und damit durch die kleinste Strukturbreite der Zonenplatte limitiert. Um maximale Auflösung zu erreichen, ist jedoch im Gegensatz zum Transmissionsmikroskop eine kohärente Beleuchtung der Zonenplatte nötig. Als Quelle für Rastermikroskope eignet sich daher besonders hochbrillante Undulatorstrahlung (siehe Abs. 2.I.I). Ist die Kohärenz der Quelle nicht ausreichend, wird stattdessen eine Kreisblende verkleinert in die Objektebene abgebildet, denn gemäß Van-Cittert-Zernike-Theorem (siehe Gl. (3.29)) ist der Grad der räumlichen Kohärenz mit der Quellgröße verknüpft. Die Kohärenz lässt sich so über den Durchmesser der Blende anpassen. Die Blende verringert allerdings die nutzbare Intensität der Quelle. Außerdem wird das $\mathrm{Ob}-$ jekt punktweise abgetastet, sodass die Aufnahmedauer mit einem Rastermikroskop etwa eine Größenordnung länger ist als bei einem Transmissionsmikroskop (wenige Minuten statt Sekunden). Daraus ergeben sich deutlich höhere Anforderungen an die Stabilität eines Rastermikroskops, um zeitliche Veränderungen wie Intensitätsschwankungen der 
Quelle, Vibrationen des mechanischen Aufbaus oder thermische Einflüsse zu minimieren. Dagegen sind Aberrationen reduziert, und das Objekt wird in einem Rastermikroskop einer geringeren Strahlenbelastung (siehe Abs. 3.1.5) ausgesetzt, da nur eine fokussierende und keine zusätzliche abbildende Optik benötigt wird. Ein weiterer Vorteil gegenüber dem Transmissionsmikroskop ist, dass sich Objekte nicht nur im Absorptions- und Phasenkontrast ${ }^{2}$ abbilden lassen, sondern mit geeigneten Detektoren gleichzeitig auch vom Objekt emittierte Fluoreszenzstrahlung oder Elektronen detektiert werden können. Rastermikroskope eignen sich daher sehr gut für die Kombination von Röntgenbildgebung und z. B. Fluoreszenz- oder Fotoelektronenspektroskopie, ohne dass die Energie der Röntgenstrahlung variiert werden muss [13]. Mit Transmissionsmikroskopen hingegen lassen sich Absorptionsspektren nur messen, wenn das Objekt bei verschiedenen Photonenenergien abgebildet wird. Das Thema Spektromikroskopie wird in Kapitel 5 behandelt.

Transmissions- und Rasterröntgenmikroskope wurden in den letzten Jahrzehnten stetig weiterentwickelt und werden in einer Vielzahl unterschiedlicher Varianten an zahlreichen Synchrotronstrahlquellen eingesetzt. Die zahlreichen Ergebnisse und daraus gewonnenen Erkenntnisse sind seit 1983 bis heute in den Veröffentlichungen von Konferenzen zur Röntgenmikroskopie dokumentiert [161-173].

\section{LINSENLOSE ABBILDUNGSVERFAHREN}

Im Spektralbereich weicher Röntgenstrahlung konnte mit Rastermikroskopen bisher eine räumliche Auflösung von etwa $10 \mathrm{~nm}$ erreicht werden [123, 149]. Das Auflösungsvermögen der besten Mikroskope liegt damit um einen Faktor 4 bis 10 über dem theoretischen Limit von etwa $\lambda / 2$ und ist demnach nicht durch Wellenlänge der Röntgenstrahlung begrenzt, sondern durch die verwendeten Zonenplatten. Als Alternative zu den „klassischen" Röntgenmikroskopen wurden deshalb in den letzten 20 Jahren "linsenlose“ Abbildungsverfahren erarbeitet, mit denen im Prinzip ein beugungsbegrenztes Auflösungsvermögen von 1 bis $2 \mathrm{~nm}$ möglich ist [174]. Die kohärente diffraktive Röntgenbildgebung (engl. coherent $x$-ray diffractive imaging, CXDI, meist nur CDI) nutzt gezielt die hohe Brillanz und Kohärenz von Synchrotronen der 3. Generation und Freie-Elektronen-Laser sowie die phasenschiebenden Eigenschaften der Objekte aus. Das Verfahren wurde für Röntgenstrahlung von Sayre et al. entwickelt [175, 176]. Das Objektiv des Röntgenmikroskops wird dabei quasi durch einen Algorithmus ersetzt. Eine CDI-Messung besteht somit im Wesentlichen aus drei Schritten:

i) Das Objekt wird mit monochromatischer, kohärenter Röntgenstrahlung beleuchtet und das Beugungsbild im Fernfeld detektiert.

2 Eine Übersicht zu Phasenkontrastverfahren findet sich z. B. in Referenz [119]. 
ii) Da auf diese Weise nur die Intensität - aber nicht die Phase - gemessen werden kann, wird diese mit iterativen Algorithmen berechnet.

iii) Das unbekannte Objekt wird mittels inverser Fouriertransformation rekonstruiert.

Experimentell realisiert wurde die kohärente diffraktive Röntgenbildgebung erstmals 1999 von Miao et al., die mit einer Auflösung von etwa $75 \mathrm{~nm}$ einzelne Goldkügelchen rekonstruierten [177]. Dank der stetigen Weiterentwicklung der Algorithmen ist es seitdem möglich, eine Vielzahl verschiedener Objekte (Nanopartikel, Nanokristalle, Zellen ...) zwei- und dreidimensional zu untersuchen. Eine Übersicht bisheriger Arbeiten und eingesetzter Algorithmen findet sich z. B. in den Referenzen [178] und [13].

Die größte Herausforderung in der kohärenten Röntgenbildgebung besteht in der Rekonstruktion der Phase. Da die meisten Objekte von Interesse nicht periodisch sind, müssen für die Algorithmen zusätzliche Informationen berücksichtigt werden. Typischerweise wird dazu Vorwissen über die Größe des Objekts genutzt oder das Objekt mit einem kollimierten Strahl beleuchtet. Wichtig ist in beiden Fällen, dass das Objekt isoliert ist („finite support constraint") [174]. Um die Phase für eine bestimmte Bildfeldgröße aus einem Beugungsbild zu bestimmen, muss die Pixelgröße des Detektors im Fernfeld daher invers zur Objektgröße skalieren. In der Praxis führt dies zu einigen Schwierigkeiten, weil ein Objekt zunächst mit einem großen Bildfeld möglichst in Echtzeit angeschaut werden soll. So werden interessante Bereiche des Objekts identifiziert, die anschließend mit hoher Auflösung abgebildet werden. Darüber hinaus wird für die Algorithmen angenommen, dass die Objekte entweder nur die Phase verändern oder die Röntgenstrahlung ausschließlich absorbiert wird. Gerade für weiche Röntgenstrahlung treten in den allermeisten Fällen aber immer beide Prozesse auf (siehe Abs. 3.I.I). Um diese Einschränkungen zu überwinden, werden mehrere Beugungsbilder von unterschiedlichen, aber überlappenden Regionen des Objekts aufgenommen („overlapping constraint"). Dazu wird das Objekt mit einem schmalen Strahl beleuchtet und anschließend abgerastert. Dabei ist die Schrittweite etwa fünf- bis zehnmal kleiner als der Strahldurchmesser. Das mehrfache Abtasten gleicher Objektbereiche entspricht im Fourierraum einer Faltung zwischen der Fouriertransformierten der begrenzten Beleuchtungsfunktion und des Beugungsbildes [179]. Dieses Verfahren wird daher als Ptychographie (aus dem Griechischem $\pi \tau v \times \eta-$ falten) bezeichnet und geht auf Walter Hoppe zurück [180], der es 1969 zuerst für die Beugung von Elektronen postulierte. Mit Röntgenstrahlung wurde erstmalig ein Objekt (Fresnel'sche Zonenplatte) von Rodenburg et al. 2007 ptychographisch abgebildet [181]. Die Qualität der Rekonstruktion hängt dabei von einer exakten Kenntnis der Beleuchtungsfunktion 
$a b$, die üblicherweise aber nicht gegeben ist. Thibault et al. erweiterten deshalb die bestehenden Algorithmen, um die Abbildungsqualität zu erhöhen und gleichzeitig die Beleuchtungsfunktion $\mathrm{zu}$ rekonstruieren [182]. Damit wurde die Ptychographie kompatibel zur Rastermikroskopie, bei der die Röntgenstrahlung auf das Objekt fokussiert wird, ohne dass die Beleuchtungsfunktion genau bekannt ist. Die räumliche Auflösung der Rastermikroskope lässt sich so deutlich über die Fokusgröße hinaus verbessern. Seitdem gibt es zahlreiche Ansätze, die Ptychographie weiterzuentwickeln. Neben der Abbildung von Objekten mit geringem Phasenschub [183] und der Erweiterung zur dreidimensionalen Rekonstruktion (Nanotomographie) [184] zählt dazu auch die Kombination mit spektroskopischen Verfahren $[185,186]$. Weitere Varianten und Anwendungsbeispiele sind in Referenz [179] aufgeführt.

Eine weitere Möglichkeit zur „linsenlosen“ Abbildung eines Objekts bietet die In-Line-Holographie [9] oder die Röntgenholografie (engl. fourier transform holography), die erstmals 1992 von McNulty et al. mit einer Auflösung einiger zehn Nanometer realisiert wurde [187]. Wie bei der diffraktiven Röntgenbildgebung muss das Objekt mit kohärenter Strahlung beleuchtet werden. Die gestreute Welle interferiert dann mit einer bekannten Referenzwelle, die von einer kleinen Apertur neben dem Objekt („,off-axis“) ausgeht. Das entstehende Hologramm wird detektiert und das Objekt durch Fouriertransformation des Interferenzmusters bestimmt. Im Vergleich zu CDI oder Ptychografie ist die Rekonstruktion damit deutlich einfacher. Allerdings ist die Strahlenbelastung höher und die Bildqualität aufgrund der Faltung mit der Referenzwelle verringert. Anwendung finden holografische Methoden z. B. in der Analyse magnetischer Domänen [188], aber auch der Abbildung biologischer Zellen [10]. Weitere Beispiele finden sich in Referenz [13].

\subsubsection{DOSIS UND STRAHLENSCH ÄDEN}

Die Absorption weicher Röntgenstrahlung führt in Materie aufgrund der hohen Photonenenergie $\mathrm{zu}$ einer Ionisation der Atome. Dadurch werden u. a. chemische Bindungen zerstört und insbesondere bei biologischen Objekten treten morphologische Veränderungen auf. Damit das Objekt während der gesamten Aufnahme intakt bleibt, darf die Strahlendosis daher einen kritischen Wert nicht übersteigen. Die Dosis gibt dabei die über die gesamte Belichtungszeit absorbierte Energie pro bestrahlter Masse an und wird in der Einheit Gray $(1 \mathrm{~Gy}=1 \mathrm{~J} / \mathrm{kg})$ gemessen. Die aufgenommene Dosis hängt gemäß Lambert-Beer'schem Gesetz (siehe Gl. (3.2)) zum einen von der Dicke des Objekts ab. Zum anderen skaliert die Dosis invers zur vierten Potenz der räumlichen Auflösung [189]: Je besser die räumliche Auflösung ist, desto kleiner ist das Probenvolumen, aus dem die Photonen zum Detektor gelangen. Neben den 
verwendeten Röntgenoptiken ist in der Transmissionsmikroskopie die maximale räumliche Auflösung demnach auch durch etwaige Strahlenschäden limitiert. Schneider berechnete, dass Dosen von $10^{5}$ bis $10^{8} \mathrm{~Gy}$ notwendig sind, um 50 bis $10 \mathrm{~nm}$ große Strukturen (z. B. Proteine) in wässriger Umgebung mit weicher Röntgenstrahlung in einem Transmissionsmikroskop abbilden zu können [190]. Biologische Zellen in wässriger Umgebung erleiden jedoch bereits ab einer Dosis von $10^{4} \mathrm{~Gy}$ schwere Schäden. Hauptursache sind freie Radikale, die aufgrund der Wechselwirkung der Röntgenstrahlung mit dem Wasser entstehen.

Um Strukturveränderungen zu verhindern, müssen die Objekte deshalb entweder getrocknet und anschließend in ein Polymer eingebettet oder in wässrigem Zustand eingefroren werden. Bei der Kryofixierung werden die Objekte mit mehreren $1000 \mathrm{~K} / \mathrm{s}$ auf eine Temperatur von etwa $136 \mathrm{~K}$ abgekühlt. Im Vergleich zu anderen Methoden werden dadurch z. B. Zellen in einem Zustand stabilisiert, der dem lebender Zellen am ähnlichsten ist. So präparierte kryogene Proben halten einer Dosis von bis $\mathrm{zu} 10^{10} \mathrm{~Gy}$ stand. Auch wenn die biochemischen Verbindungen innerhalb der Zelle verändert werden, ändert sich der Bildkontrast nicht, da sich die Atome innerhalb der gefrorenen Probe nicht bewegen können [125]. Alternativ lässt sich die Strahlenbelastung eines Objekts um etwa eine Größenordnung verringern, wenn die Probe im Phasen- und nicht im Absorptionskontrast abgebildet wird. Zudem ist die Dosis in Rastermikroskopen geringer, da die abbildende Optik hinter dem Objekt entfällt. Für höchste Auflösungen von weniger als $10 \mathrm{~nm}$ sind hinsichtlich der Strahlenschäden linsenlose Verfahren am besten geeignet. An Freie-Elektronen-Lasern wird dank der hohen Brillanz die dafür benötigte Dosis erreicht und aufgrund der ultrakurzen Röntgenpulse $(<$ 10-200 fs) wird das Signal detektiert, bevor das Objekt zerstört wird („diffraction before destruction“) [191, 192].

\subsection{STAND DER FORSCHUNG}

Messzeit mit synchrotronbasierten Röntgenmikroskopen ist aufgrund der geringen Anzahl entsprechender Strahlquellen stark begrenzt. Darüber hinaus ist z. B. bei der Untersuchung biologischer Objekte ein direkter Zugang zu den Mikroskopen notwendig, um Proben unmittelbar nach ihrer Präparation abbilden zu können. Laborgeräte sind deshalb eine entscheidende Voraussetzung, um die Röntgenmikroskopie über die Benutzergemeinschaft von Synchrotronen hinaus zu etablieren.

Kompakte Mikroskope für den Spektralbereich weicher Röntgenstrahlung basieren entweder auf Entladungs- oder laserinduzierten Plasmaquellen. Davon abgesehen, sind sie wie Transmissionsmikroskope an Synchrotronen aufgebaut (siehe Abs. 3.1.4) und unterscheiden sich neben der Quelle im Wesentlichen nur durch die Art der Kondensoroptik. 
Im Folgenden werden die verschiedenen Konzepte und wichtigsten Anwendungen bisher realisierter Laborröntgenmikroskope vorgestellt.

Bereits 1994 entwickelten Nakayama et al. ein Röntgenmikroskop auf der Basis einer laserinduzierten Plasmaquelle mit einem Festkörpertarget aus Kohlenstoff [193]. Ein elliptischer Multischichtspiegel filterte die 3,37 nm-Linie aus dem breitbandigen Emissionsspektrum heraus und fokussierte die Strahlung auf das Objekt. Das Auflösungsvermögen war bei einer maximalen Vergrößerung von 286 allerdings nur etwas besser als die eines Lichtmikroskops. Die unvermeidliche Entstehung von Debris und die schwierige Bereitstellung von neuem Targetmaterial verhindern zudem einen stabilen Langzeitbetrieb des Mikroskops.

Im gleichen Jahr demonstrierten Rudolph et al. mit ihrem Aufbau ein Auflösungsvermögen von 100 bis $150 \mathrm{~nm}$ [194]. Dazu nutzten sie eine Gasentladungsquelle in Kombination mit elliptischem Kondensorspiegel und hochauflösender Zonenplatte. Problematisch ist dabei die geringe Stabilität der Entladungsquelle. Es ist außerdem nicht möglich, ein Auflösungsvermögen deutlich unter $100 \mathrm{~nm}$ zu erreichen, weil die kleine spektrale Bandbreite der Quelle $(E / \Delta E=100-300) \mathrm{zu}$ chromatischen Aberrationen führt.

Das erste Laborröntgenmikroskop, mit dem reproduzierbar hochauflösende Abbildungen wasserfreier Objekte mit hohem Signal-RauschVerhältnis möglich waren, wurde 1999 in der Arbeitsgruppe von Hans Hertz (Universität Stockholm) realisiert [21]. Als Quelle weicher Röntgenstrahlung dient ein laserinduziertes Plasma. Das Target besteht aber nicht aus festem Material, sondern aus mikroskopisch kleinen Ethanoltröpfchen. Dadurch wird zum einen deutlich weniger Debris freigesetzt. Zum anderen kann das Targetmaterial auch bei hohen Repetitionsraten $(>100 \mathrm{~Hz})$ effizient nachgeführt werden, um einen stabilen Betrieb der Quelle zu gewährleisten. Das Mikroskop wird bei der intensivsten Linie des Spektrums bei einer Wellenlänge von 3,37 $\mathrm{nm}$ betrieben. Als Kondensor dient ein sphärischer Multischichtspiegel (Reflektivität 0,5\%). Das Objekt wird mit einer phasenschiebenden Zonenplatte (Effizienz ca. 7,3\%) mit bis zu 1000facher Vergrößerung auf einen gedünnten, rückseitig belichteten CCD-Chip abgebildet. Die räumliche Auflösung beträgt bei Belichtungszeiten von wenigen Minuten etwa $60 \mathrm{~nm}$.

Darauf aufbauend wurde das Laborröntgenmikroskop kontinuierlich verbessert: Mit einem optimierten Multischichtspiegel höherer Reflektivität konnte zunächst die Belichtungszeit auf ein Zehntel verringert werden [133]. Darüber hinaus wurde das Ethanoltarget durch einen kontinuierlichen Strahl aus flüssigem Stickstoff ersetzt [195]. Durch das veränderte Emissionsspektrum der Plasmaquelle können die Objekte folglich mit einer kürzeren Wellenlänge von 2,48 nm abgebildet werden. Dadurch wird etwa fünfmal weniger Strahlung von der Probe absorbiert, sodass auch Zellen in wässriger Umgebung mit einer Dicke von bis zu 
$10 \mu \mathrm{m}$ untersucht werden konnten. Der Probenhalter befand sich dazu in einer Heliumatmosphäre. Ebenso wurden Abbildungen mit einer Auflösung von $25 \mathrm{~nm}$ [196] und im Zernike-Phasenkontrast demonstriert [197]. Eine Verbesserung der Quellintensität wurde ferner durch ein neues Lasersystem hoher Leistung von 60 bis 200 W (Pulsenergie 100 m J, Repetitionsrate bis $2 \mathrm{kHz}$ ) erreicht [198], mit dem die mittlere Brillanz des Plasmas um einen Faktor 5 bis 15 gesteigert wurde. Parallel zu der Entwicklung in der AG Hertz wird am „Berlin Laboratory for innovative Xray Technologies - BLiX" (Technische Universität Berlin und Max-BornInstitut für Nichtlineare Optik und Kurzzeitspektroskopie) ebenfalls auf Basis dieses Lasersystems und flüssigen Stickstofftargets ein Laborröntgenmikroskop aufgebaut [199, 200].

Das Laborröntgenmikroskop der AG Hertz wurde bereits für verschiedene Anwendungen genutzt. So wurde etwa die Struktur von Kolloiden, Tonmineralen und Bodenkolloiden in ihrem natürlichen, wässrigen $\mathrm{Zu}$ stand analysiert [201, 202]. Des Weiteren konnten 2009 erstmals strahlenresistente Objekte (Kieselalgen) dreidimensional abgebildet werden [203]. Um Strahlenschäden bei der tomografischen Aufnahme biologischer Proben zu vermeiden, wurde das Mikroskop zudem um einen drehbaren Probenhalter für kryofixierte Objekte erweitert. Damit gelang 2011 die zwei- und dreidimensionale Abbildung intakter Zellen [204, 205]. Die Bildqualität ist vergleichbar zu synchrotronbasierten Mikroskopen, obwohl die Messzeit von etwa 20 min für die Aufnahme aller Bilder zur dreidimensionalen Rekonstruktion der Zellen lediglich um einen Faktor 5 länger ist [134].

Für kompakte Röntgenmikroskope werden auch Gasentladungsquellen genutzt. Aufgrund des großen Plasmadurchmessers von über $1 \mathrm{~mm}$ ist die mittlere Brillanz trotz hoher Repetitionsraten (bis zu $1 \mathrm{kHz}$ ) jedoch bis zu drei Größenordnungen geringer als bei laserinduzierten Plasmen mit Flüssigtarget. Zusätzlich erodieren die Elektroden der Entladungsquelle. Dies führt einerseits zur Produktion von Debris, andererseits vermindert es die Quellstabilität. Ein dauerhafter Betrieb der Gasentladungsquellen ist daher problematisch. Folglich wurden bisher nur zwei Mikroskope realisiert, die aber ausschließlich zur Abbildung von Testobjekten und nicht für Anwendungen eingesetzt wurden [22, 76].

Trotz der bisherigen Erfolge in der Entwicklung und Anwendung von Laborröntgenmikroskopen besteht die Notwendigkeit, die Mikroskope noch kompakter $\mathrm{zu}$ bauen und sie weiter $\mathrm{zu}$ vereinfachen. Dabei ist besonders wichtig, einen stabilen Betrieb der Quelle zu gewährleisten und die Bedienerfreundlichkeit des Mikroskops sicherzustellen. Die unvermeidliche Debrisproduktion bei laserinduzierten Plasmen mit festen oder flüssigen Targets sowie bei Entladungsquellen steht diesen Anforderungen jedoch entgegen. Zudem ist ein erheblicher experimenteller und technischer Aufwand nötig, um z. B. ein stabiles Kryotarget mit flüs- 
sigem Stickstoff zu realisieren. Als Alternative wurden daher laserinduzierte Plasmen mit gasförmigen Targets entwickelt (siehe Abs. 2.3.2 und 2.3.3). Im Rahmen dieser Arbeit wurde erstmals gezeigt, dass Röntgenmikroskopie mit einem besseren Auflösungsvermögen als für Lichtmikroskope auch unter Verwendung dieser stabilen, nahezu debrisfreien und sehr kompakten Quelle möglich ist [206, 207]. Um in Testobjekten Strukturen mit einer Größe von etwa $50 \mathrm{~nm}$ aufzulösen, sind aufgrund der geringen Brillanz allerdings Belichtungszeiten zwischen 30 und $120 \mathrm{~min}$ notwendig.

Nahezu gleichzeitig zu den Arbeiten in der Abteilung Optik / Kurze Wellenlängen des Laser-Laboratoriums Göttingen wurde in der Arbeitsgruppe von Henryk Fiedorowicz (Technische Militärakademie Warschau) ebenfalls ein kompaktes Mikroskop basierend auf einem laserinduzierten Plasma mit gasförmigen Target und vergleichbarer Leistungsfähigkeit aufgebaut [208, 209].

\subsection{LABORRÖNTGENMIKROSKOP}

Im Zuge dieser Arbeit wurde ein Mikroskop zur hochauflösenden Abbildung von Objekten mit weicher Röntgenstrahlung entwickelt, dessen Aufbau, Funktionsweise und Leistungsvermögen in den folgenden Abschnitten beschrieben werden. Einige der Abbildungen und Ergebnisse finden sich in ähnlicher Weise in folgenden Publikationen:

M. Müller, T. Mey, J. Niemeyer und K. Mann,"Table-top soft x-ray microscope using laser-induced plasma from a pulsed gas jet," Optics Express 22(19), 023489 (2014) und

M. Müller, T. Mey, J. Niemeyer, M. Lorenz und K. Mann, "Table-top soft X-ray microscopy with a laser-induced plasma source based on a pulsed gas-jet," AIP Conference Proceedings 1764(1), 030003 (2016).

\subsubsection{MIKROSKOPAUFBAU}

Für das Laborröntgenmikroskop (siehe Abb. 3.8) wird als Quelle ein mit einem Nanosekundenlaser (Quantel, Wellenlänge 1064 nm, Pulslänge $10 \mathrm{~ns}$, Pulsenergie bis $600 \mathrm{~mJ}$ ) induziertes Plasma mit gasförmigem Stickstoff genutzt (siehe Abs. 2.3.3 und 2.3.4). Ansonsten besteht das Mikroskop aus einem elliptischen Kondensorspiegel (Rigaku), einer Zonenplatte und einer Kamera (Roper Scientific, 1024 x 1024 Pixel, Pixelgröße $13 \mu \mathrm{m}$ bzw. Andor, 2048 x 2048 Pixel, Pixelgröße 13,5 $\mu \mathrm{m}$ ) mit rückseitig belichtetem, gedünntem CCD-Chip. Zudem befindet sich ein $200 \mathrm{~nm}$ dicker Titanfilter im Strahlengang. Sein Einfluss ist in Abbildung 3.9 dargestellt: Strahlung unterhalb der Absorptionskante des Titans $(\lambda=2,7 \mathrm{~nm})$ 


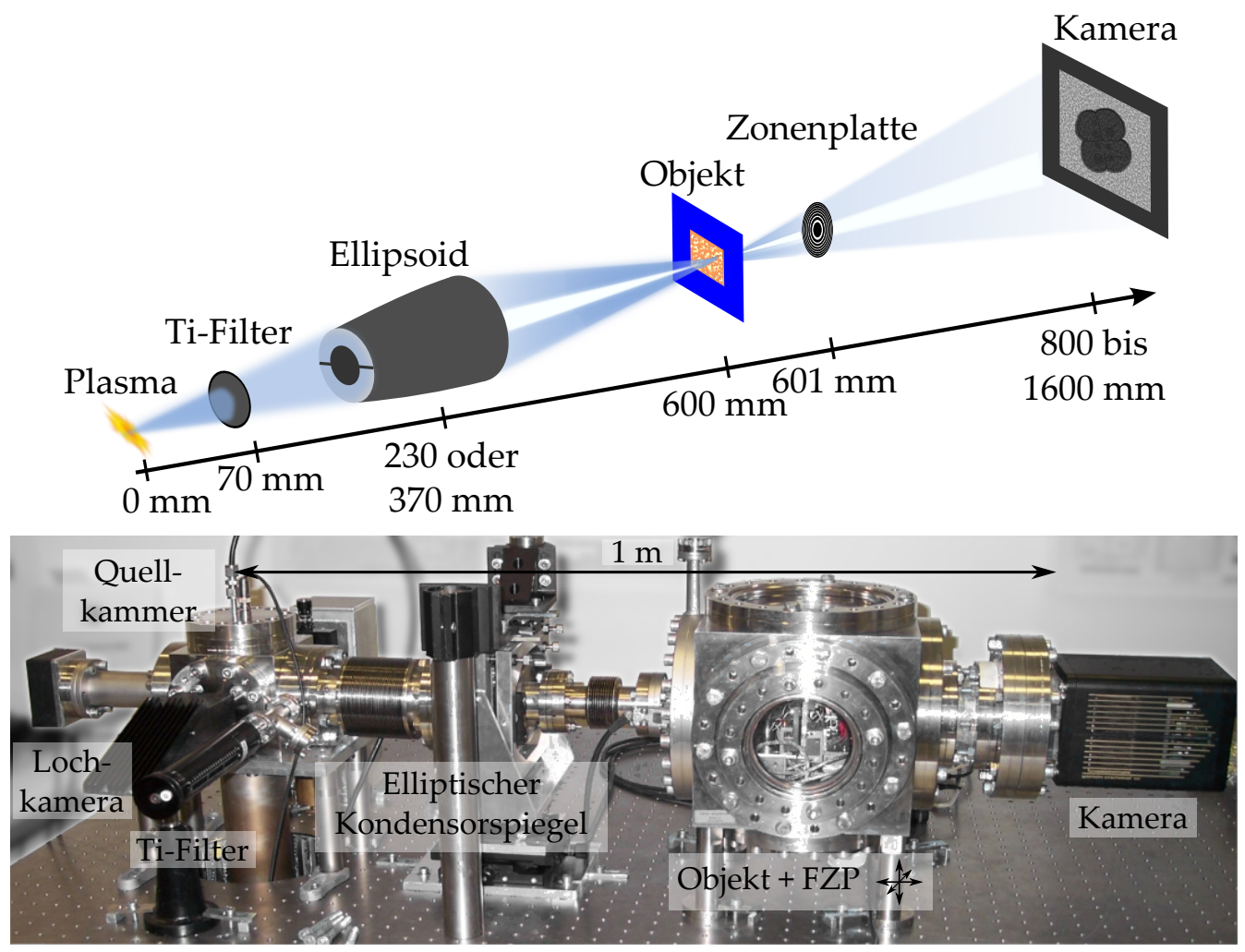

Abbildung 3.8: Schematische Darstellung (oben) und Foto des Laborröntgenmikroskops (unten)

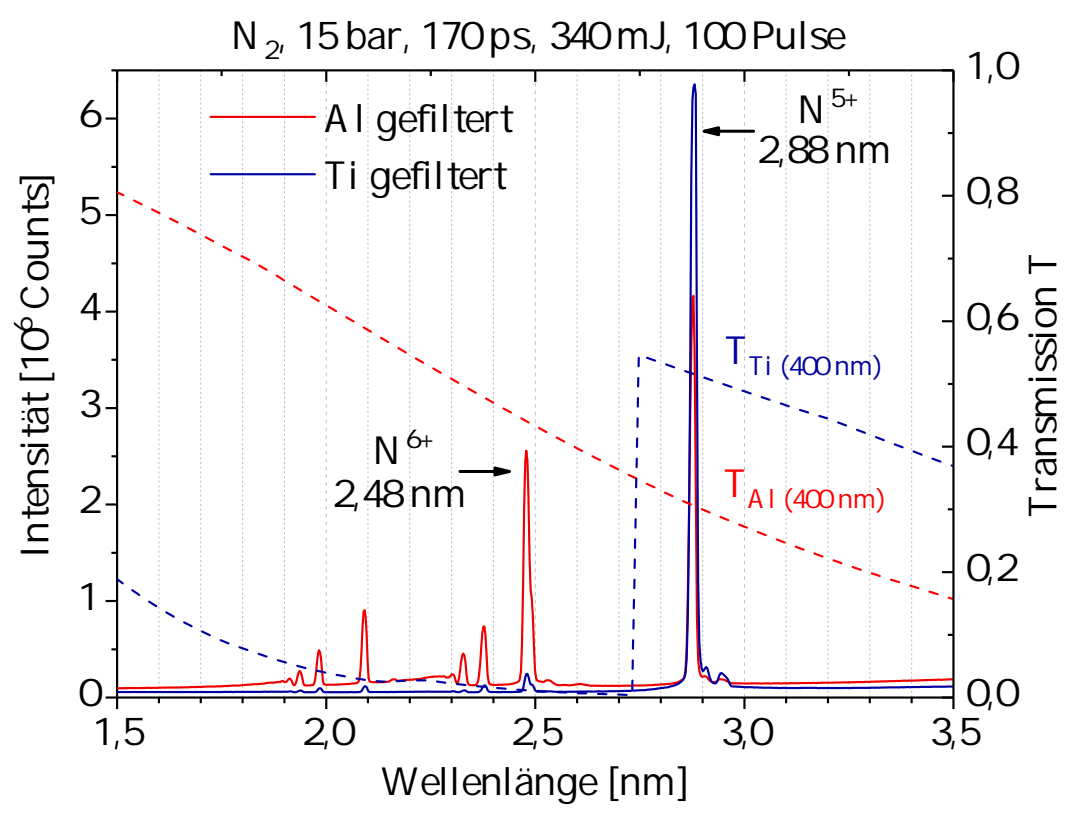

Abbildung 3.9: Mit Aluminium- bzw. Titanfilter (Dicke $400 \mathrm{~nm}$ ) gemessenes Emissionsspektrum von Stickstoff (Transmission der Filter aus Ref. [120]) 
sowie sichtbares Licht und gestreute Laserstrahlung werden vom Filter geblockt. So wird sichergestellt, dass das Objekt nur mit monochromatischer Röntgenstrahlung der Wellenlänge $\lambda=2,88 \mathrm{~nm}$ beleuchtet wird. Wenn nicht anders angegeben, wurden die Messungen bei einem Stickstoffdruck von 25 bar durchgeführt.

Der CCD-Chip zeichnet sich durch seine hohe Sensitivität und das geringe Ausleserauschen aus. Der Nachteil des Detektors ist sein limitierter Dynamikumfang von lediglich einigen $10^{3}$ Photonen pro Pixel, der durch den hohen Konversionsfaktor (118 Elektronen pro Röntgenphoton bei $\lambda=2,88 \mathrm{~nm}$ ) und die begrenzte Aufnahmekapazität von etwa $10^{5}$ Elektronen pro Pixel bedingt ist.

Um das thermische Rauschen während der Bildaufnahme zu minimieren, wird der Chip auf $-40^{\circ} \mathrm{C}$ gekühlt. Dennoch werden - insbesondere bei langen Belichtungszeiten - Elektronen thermisch generiert. Diese werden von den einzelnen Pixeln unterschiedlich stark akkumuliert, sodass auf dem CCD-Chip eine zeitabhängige Dunkelstromstruktur entsteht. Durch Subtraktion eines Dunkelbildes (ohne Bestrahlung, ansonsten gleiche Aufnahmebedingungen) wird diese und der konstante Offset der Ausleseelektronik in den Mikroskopbildern korrigiert.

\section{BELEUCHTUNGSSYSTEM}

Die vom Plasma emittierte Strahlung wird von einem nickelbeschichteten elliptischen Kondensorspiegel (Rigaku, Länge 100 mm, Brennweite $300 \mathrm{~mm}$, siehe Abb. 3.10) aufgesammelt und in die Objektebene fokussiert. Die Einfallswinkel $\theta$ zur Spiegeloberfläche liegen dabei zwi-

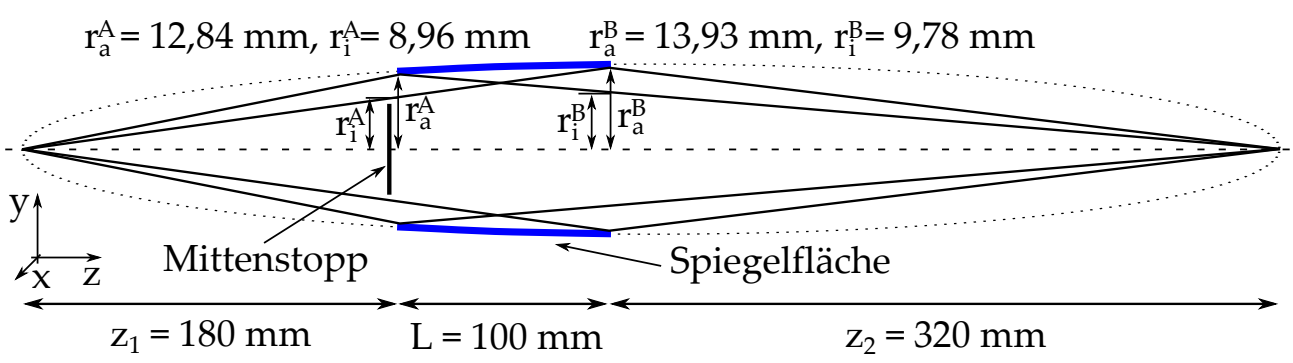

Abbildung 3.10: Skizze mit Spezifikationen des elliptischen Kondensorspiegels

schen 1,8 und $2,5^{\circ}$ und damit unter dem kritischen Winkel $\theta_{\mathrm{c}}^{\mathrm{Ni}}=6,5^{\circ}$ für Totalreflexion. Die Reflektivität des Spiegels beträgt dennoch keine $100 \%$. Verringert wird sie einerseits durch die Absorption weicher Röntgenstrahlung in Nickel $(\beta / \delta=0,28$ bei $\lambda=2,88 \mathrm{~nm})$ sowie andererseits aufgrund der Oberflächenrauheit von etwa 0,5 nm (Herstellerangabe). Trotzdem ist für den Kondensor eine theoretische Reflektivität von über $50 \% \mathrm{zu}$ erwarten (siehe Abb. 3.4). Experimentell lässt sich diese Annahme überprüfen, indem mit einer kalibrierten Photodiode (IRD AXUV10o) die vom Plasma abgestrahlte Energie jeweils mit 


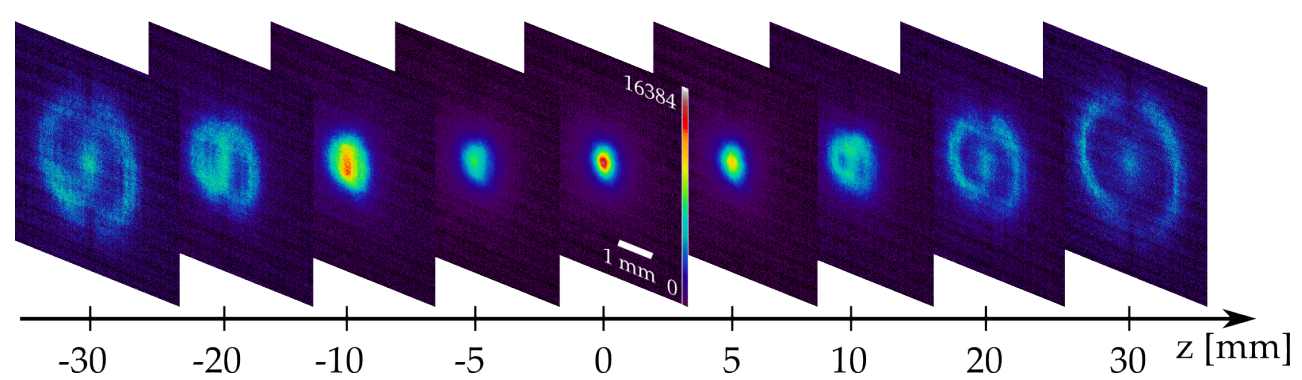

Abbildung 3.11: Intensitätsverteilung ( $\lambda=2,88 \mathrm{~nm}$, Mittelung 20 Pulse, Konfiguration A) entlang der optischen Achse hinter dem Kondensorspiegel. Die Intensität an den Positionen $\pm 10 \mathrm{~mm}, \pm 20 \mathrm{~mm}$ und $\pm 30 \mathrm{~mm}$ wurde für eine bessere Sichtbarkeit der Profile jeweils um den Faktor 2, 3 bzw. 4 erhöht.

und ohne Kondensorspiegel gemessen wird. Dabei sind die unterschiedlichen Raumwinkel zu berücksichtigen, in die die Strahlung emittiert wird. Ohne Spiegel ergibt sich ein Wert von $120 \mu \mathrm{J} \mathrm{sr}^{-1}$ pro Puls, mit Spiegel wurde eine Energie von $34 \mu \mathrm{J} \mathrm{sr}^{-1}$ gemessen. Die Reflektivität des elliptischen Kondensorspiegels beträgt demnach bei $\lambda=2,88 \mathrm{~nm}$ knapp $30 \%$ und weicht somit um einen Faktor 2 vom theoretischen Wert ab. Die wahrscheinlichste Ursache dafür ist, dass die Rauheit über die gesamte Spiegeloberfläche größer ist als die vom Hersteller genannten $0,5 \mathrm{~nm}$. Schon geringfügig höhere Werte führen aufgrund der kleinen Wellenlänge zu einer deutlichen Zunahme gestreuten Lichts und damit zu einer verminderten Reflektivität des Spiegels.

Ein Mittenstopp (Radius $6 \mathrm{~mm}$ ) am Kondensorspiegel verhindert die direkte Beleuchtung eines zentralen Bereichs auf dem Detektor, sodass die Abbildung des Objekts nicht von anderer Strahlung überlagert wird. Die aus dieser Hohlkegelbeleuchtung resultierende Intensitätsverteilung wurde entlang der optischen Achse hinter dem elliptischen Kondensorspiegel mit einer Kamera mit Phosphor (ProxiVision, P43, Schichtdicke $5 \mu \mathrm{m}$ ) beschichtetem CCD-Chip (Sony ICX285, Pixelgröße 6,45 $\mu \mathrm{m}$, 1280 x 1024 Pixel) gemessen (siehe Abb. 3.II). Zusätzlich zum Titanfilter befand sich eine $4 \mu \mathrm{m}$ dicke Polypropylenfolie (Transmission 0,2\% bei $\lambda=2,88 \mathrm{~nm}$ ) im Strahlengang, um eine Überbelichtung der Kamera im Fokus zu verhindern. Die ringförmige Struktur für große Abstände zur Fokusposition $(z=0 \mathrm{~mm})$ ist deutlich sichtbar.

Der Kondensorspiegel kann entweder näher zur Quelle (Konfiguration A) oder näher zum Objekt (Konfiguration B) eingesetzt werden (siehe Abb. 3.10). Die Austrittsapertur beträgt dann $N A_{\text {Kond }}^{\text {Aus, }}=0,044$ bzw. $N A_{\text {Kond }}^{\text {Aus, }}=0,071$. Im Fall A wird das Plasma dementsprechend um den Faktor $370 / 230=1,6$ vergrößert, im Fall B um den Faktor 1,6 verkleinert in die Objektebene abgebildet. Die Intensitätsverteilung im Fokus ist für beide Positionen des Kondensors in Abbildung 3.12 gezeigt. Um die Fokusgröße zu bestimmen, wurde an die vertikalen und horizontalen Querschnitte eine Lorentzkurve angepasst. Dadurch wer- 

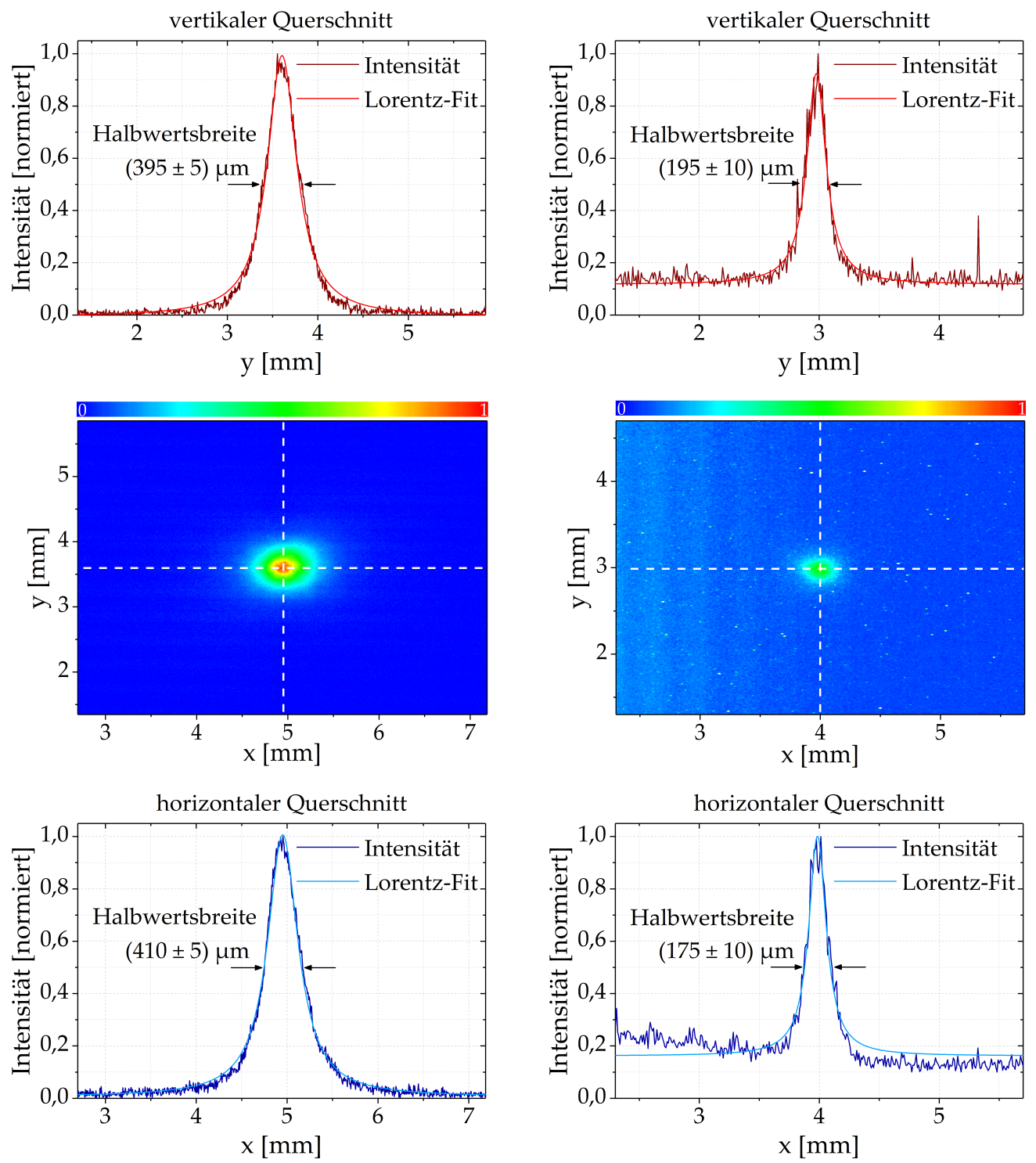

Abbildung 3.12: Intensitätsverteilung (Mitte) im Fokus des Kondensorspiegels für Konfiguration A (links) und Konfiguration B (rechts). An die vertikalen (oben) und horizontalen (unten) Querschnitte wurde zur Berechnung der Halbwertsbreiten jeweils eine Lorentzfunktion angepasst. Die Fitparameter sind in Tabelle A.2 aufgelistet. Die Absolutwerte der Intensität sind nicht vergleichbar, da für die Messungen unterschiedliche Kameras verwendet wurden. 
den die Profile insbesondere für Intensitäten $<0,3$ besser beschrieben als z. B. mit einer Gaußfunktion, da Aberrationen des Spiegels zu einer Verbreiterung des Fokus führen. Ist das Ellipsoid näher an der Quelle, ergibt sich eine Ausdehnung (Halbwertsbreite) von $d_{x}^{A}=410(5) \mu \mathrm{m}$ und $d_{y}^{A}=395(5) \mu m$. Die Pulsenergie im Fokus wurde mit einer kalibrierten Photodiode (IRD AXUV100) zu 100 nJ gemessen. Die Energiedichte pro Puls beträgt unter Berücksichtigung der Fokusgröße damit $0,081 \mathrm{~mJ} / \mathrm{cm}^{2}\left(1,2 \times 10^{4}\right.$ Photonen $\left./ \mu \mathrm{m}^{2}\right)$.

Wird der Kondensorspiegel gedreht, verringert sich der Fokusdurchmesser um den Faktor 2,34 bzw. 2,03 auf $d_{x}^{B}=175(10) \mu m$ und $d_{y}^{B}=$ 195(10) $\mu \mathrm{m}$. Damit ist der Fokus bei dieser Konfiguration insbesondere in vertikaler Richtung größer als erwartet, da die Durchmesser theoretisch um den Faktor 1,6 $6^{2}=2,56$ kleiner sein müssten. Die Abweichung lässt sich auf Aberrationen des elliptischen Spiegels zurückführen. Diese tragen stärker zu einer Vergrößerung des Fokusdurchmessers bei, wenn das Plasma verkleinert abgebildet wird (Konfiguration B) [137].

Die Ellipsoidposition wirkt sich direkt auf das Leistungsvermögen des Mikroskops aus. Infolge des kleineren Fokus der Konfiguration B können von der abbildenden Optik einerseits mehr Photonen aufgesammelt werden, die zum Detektor gelangen. Andererseits ist die quellseitige Apertur geringer, sodass weniger Photonen in die Objektebene fokussiert werden. Um abzuschätzen, welcher Effekt überwiegt, wurde für beide Positionen jeweils ein Leerbild mit abbildender Optik, aber ohne Objekt aufgenommen (Vergrößerung $M=470$, Belichtungszeit $120 \mathrm{~min}$ ). Befindet sich der Kondensor näher an der Objektebene (Konfiguration B), ist die Intensität verglichen mit Position A (näher an Quelle) etwa um das 2,5fache höher. Die kleinere Fokusgröße ist demnach von Vorteil.

\section{ABBILDENDES SYSTEM}

Um das Objekt auf den Detektor abzubilden, stehen drei verschiedene Zonenplatten (ZonePlates Ltd, Parameter siehe Tab. 3.2) aus Wolfram zur Auswahl. Ihre Zonentiefe beträgt $130 \mathrm{~nm}$ und ist somit für einen optimalen Phasenschub und folglich maximale Effizienz angepasst. Nach Gleichung (3.19) ergibt sich ein theoretischer Beugungswirkungsgrad von $11,7 \%$. Da zusätzlich die Transmission ( $T \approx 0,5$ bei $\lambda=2,88 \mathrm{~nm}$ ) der $100 \mathrm{~nm}$ dicken $\mathrm{Si}_{3} \mathrm{~N}_{4}$-Trägermembran berücksichtigt werden muss, verringert sich die theoretisch erreichbare Effizienz allerdings auf etwa $6 \%$. Experimentell wurde für $\mathrm{ZP} 2$ hingegen ein Beugungswirkungsgrad von ca. $4 \%$ gemessen, indem die Anzahl der Photonen pro Pixel und Sekunde aus der Energiedichtemessung (ohne $\mathrm{ZP}_{2}$ ) mit dem Wert aus einem Leerbild (mit ZP2, ohne Objekt) verglichen wurden. Dabei wurde berücksichtigt, dass die Zonenplatte kleiner ist als der Fokus des Ellipsoids und daher nur einen Anteil der Photonen aus der Fokusebene aufsammeln kann. Die Abweichung zur theoretisch berechneten Effizienz ist 
Tabelle 3.2: Parameter der im Laborröntgenmikroskop verwendeten Zonenplatten bei $\lambda=2,88 \mathrm{~nm}$ in der ersten Beugungsordnung

\begin{tabular}{|c|c|c|c|}
\hline \multirow[t]{2}{*}{ Parameter } & \multicolumn{3}{|c|}{ Wert } \\
\hline & $\mathrm{ZP} 1$ & $\mathrm{ZP} 2$ & $\mathrm{ZP}_{3}$ \\
\hline Breite äußerster Ring $d r_{N}[n m]$ & 40 & 30 & 25 \\
\hline Durchmesser D $[\mu \mathrm{m}]$ & 80 & 190 & 120 \\
\hline Anzahl Zonen N & 500 & 1583 & 1200 \\
\hline Brennweite $f_{0}[\mu \mathrm{m}]$ & 1111,1 & 1979,2 & 1041,7 \\
\hline numerische Apertur NA & 0,036 & 0,048 & 0,058 \\
\hline Auflösungsvermögen $\delta^{\text {Rayl. }}[\mathrm{nm}]$ & 48,8 & 36,6 & 30,5 \\
\hline Schärfentiefe $\Delta z[\mu \mathrm{m}]$ & $\pm 1,11$ & $\pm 0,63$ & $\pm 0,43$ \\
\hline
\end{tabular}

zum einen durch zusätzliche Stützstrukturen (siehe Abb. 3.16) bedingt. Diese sind notwendig, um die Stabilität der Zonenplatte zu gewährleisten, führen aber zu einem höheren Anteil absorbierter Strahlung. Zum anderen wird der theoretische Wert nicht erreicht, weil die Zonen insbesondere für die kleinen Strukturbreiten vom idealen Zonenplattenbildungsgesetz (siehe Gl. (3.17)) abweichen. Die Fläche benachbarter Ringe ist daher nicht gleich groß, sodass eine Abbildung von Objekten in der zweiten Beugungsordnung möglich war. Dadurch nimmt die Effizienz in der ersten Beugungsordnung jedoch ab.

Die numerische Apertur der Zonenplatte sollte an die des Kondensors angepasst sein. Abhängig davon, in welcher Konfiguration das Ellipsoid verwendet wird, muss demnach eine passende Zonenplatte ausgewählt werden. In Konfiguration $\mathrm{A}\left(\mathrm{NA} \mathrm{A}_{\text {Kond }}^{\text {Aus, }}=0,044\right)$ eignen sich $\mathrm{ZP} 1$ und $\mathrm{ZP} 2$. Für Konfiguration $\mathrm{B}\left(\mathrm{N} A_{\mathrm{Kond}}^{\text {Aus, }}=0,071\right)$ ist $\mathrm{ZP} 3$ die beste Wahl, obwohl die numerische Apertur der Zonenplatte etwas zu klein ist. Für eine optimale NA müsste die äußerste Zonenbreite $20 \mathrm{~nm}$ statt $25 \mathrm{~nm}$ betragen. Eine solche Zonenplatte war jedoch nicht verfügbar.

Ist die numerische Apertur des Kondensors deutlich größer als die der Zonenplatte, sammelt diese fast ausschließlich vom Objekt gestreutes Licht auf. Der Beleuchtungskegel hingegen strahlt größtenteils an der Zonenplatte vorbei, sodass das Objekt im Dunkelfeldkontrast abgebildet wird. Dieses Kontrastverfahren lässt sich z. B. realisieren, wenn für die Ellipsoidposition $\mathrm{B} \mathrm{ZP}_{2}$ statt $\mathrm{ZP}_{3}$ genutzt wird.

Die Bildweite (Abstand Zonenplatte - Detektor) kann zwischen 0,2 m und $1 \mathrm{~m}$ variiert werden, sodass Objekte bis zu 1000fach vergrößert abgebildet werden können. 


\subsubsection{TESTOBJEKTE}

Das Laborröntgenmikroskop wurde zunächst zur Abbildung von Testobjekten bekannter Struktur eingesetzt, um dessen Leistungsvermögen zu charakterisieren. Das erste Objekt ist ein Kupfergitter der Periode $12,5 \mu \mathrm{m}$. Das Ellipsoid befand sich in näher an der Quelle (Position A) und als Objektiv wurde ZP2 verwendet. In Abbildung 3.13 sind die Mikroskopaufnahmen des Gitters bei 225facher Vergrößerung für verschiedene Belichtungszeiten gezeigt. Um die Bildqualität zu vergleichen, wurde gemäß Gleichung (3.5) bzw. (3.6) der Michelson-Kontrast $C_{M}$ und das Signal-Rausch-Verhältnis SNR berechnet. Dazu wurde jeweils über die Intensitätswerte innerhalb der markierten Quadrate (S: Signal, N: Rauschen) gemittelt (Daten siehe Tab. A.3). Je länger die Belichtungszeit ist,

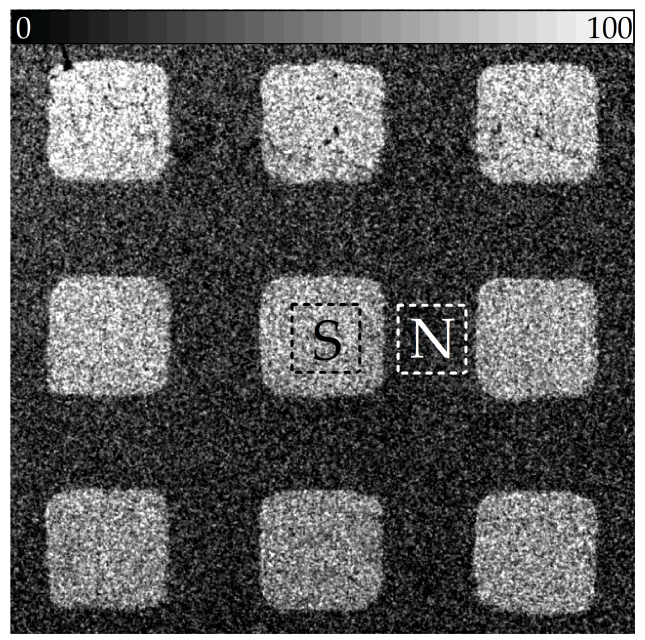

(a) $t=1 \min , C_{M}=0,22, S N R=9,4$

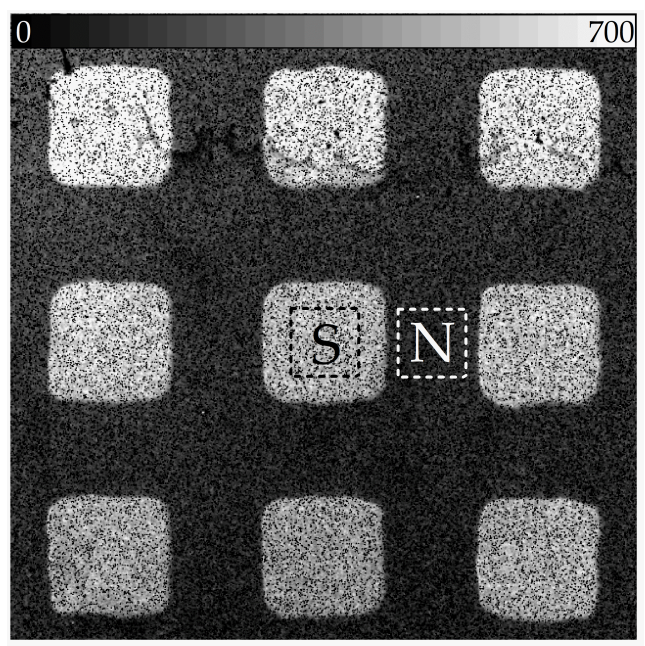

(c) $t=10 \times 1 \mathrm{~min}, \mathrm{C}_{M}=0,22, \mathrm{SNR}=7,5$

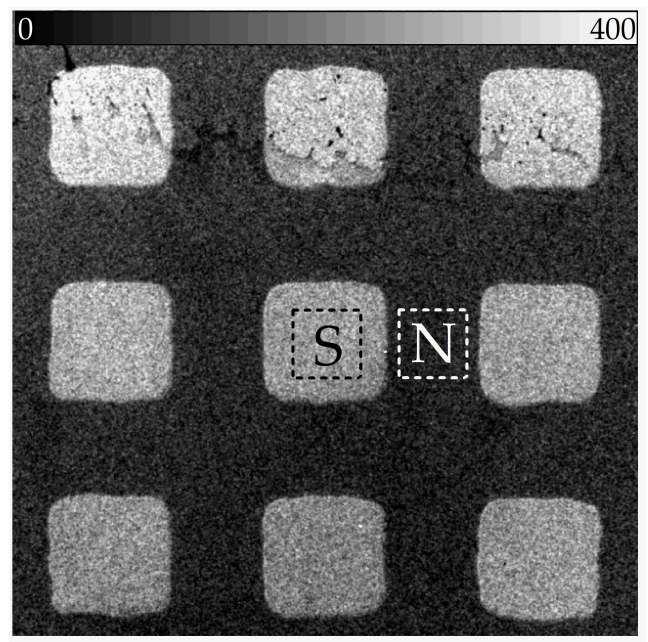

(b) $t=5 \mathrm{~min}, \mathrm{C}_{M}=0,22, \mathrm{SNR}=20,9$

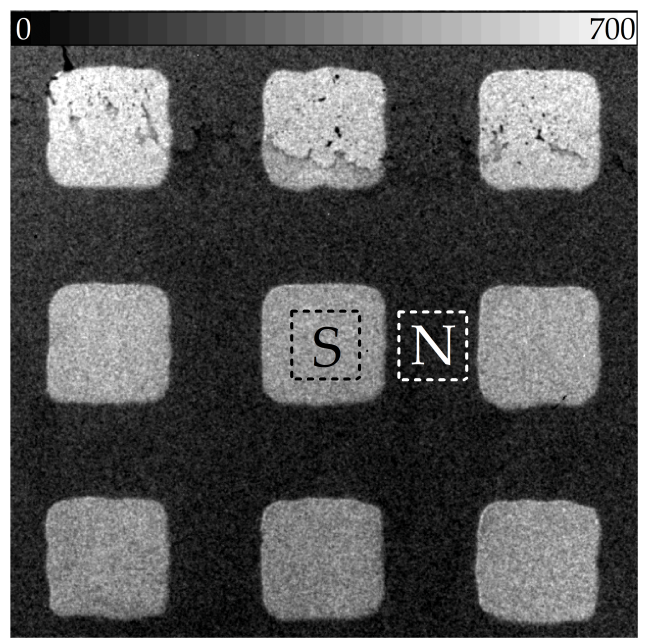

(d) $t=10 \min , C_{M}=0,21, S N R=27,0$

Abbildung 3.13: Röntgenmikroskopische Aufnahmen eines Kupfergitters ( $M=$ 225) für verschiedene Belichtungszeiten $t$. Die Gitterstege sind $5 \mu \mathrm{m}$ breit. 
desto höher ist auch das Signal-Rausch-Verhältnis. Der Kontrast hingegen bleibt bei allen Aufnahmen unverändert. Um eine Akkumulation des elektronischen Ausleserauschens zu verhindern, sollte zudem nicht mehrmals über kurze Zeiten (z. B. 10x 1 min), sondern über die Gesamtzeit integriert werden.

Ein Siemensstern eignet sich sehr gut, um unmittelbar das Auflösungsvermögen und mögliche Abbildungsfehler des Mikroskops zu ermitteln (siehe Abs. 3.I.I). Der Siemensstern (NTT-AT, Modell ATN/XRESO-50) wurde dazu zuerst für Ellipsoidposition A mit ZP2 bei einer Vergrößerung von $M=470$ abgebildet (siehe Abb. 3.14). Trotz der langen Belichtungszeit von $t=120$ min ist die Bildqualität gering. Daher sind nur einzelne der $100 \mathrm{~nm}$-Strukturen sichtbar. Für größere Strukturen ist die Abbildungsqualität jedoch richtungsinvariant.

Eine deutlich bessere Auflösung ergibt sich bei der Abbildung des Siemenssterns im Dunkelfeldkontrast. Dazu wurde das Ellipsoid in Konfiguration B (näher an Objekt) verwendet. Da die numerische Apertur des Kondensors in diesem Fall größer als die der Zonenplatte ist, gelangt fast nur vom Objekt gestreutes Licht zur Zonenplatte und damit zum Detektor. Dadurch werden die Kanten stark überhöht und auch die kleinsten Strukturen von $50 \mathrm{~nm}$ sind erkennbar.

In Abbildung 3.15 sind zwei Hellfeldaufnahmen eines zweiten Siemenssterns (ZonePlate Ltd) gezeigt, die mit $\mathrm{ZP}_{3}$ für Konfiguration $\mathrm{B}$ des Ellipsoids aufgenommen wurden. Bei einer geringen Vergrößerung von $M=175$ lassen sich bereits bei Belichtungszeiten von 5 min Strukturen bis etwa $150 \mathrm{~nm}$ auflösen. Zudem eignet sich dieser Modus, um Übersichtsbilder des Objekts über ein Bildfeld von bis zu $50 \mu \mathrm{m}$ aufzunehmen. Bei stärkerer Vergrößerung $(M=375)$ lassen sich dann 50 bis $60 \mathrm{~nm}$ große Objektdetails auflösen. Die Belichtungszeit verlängert sich aufgrund der kleineren effektiven Pixelfläche allerdings auf $30 \mathrm{~min}$. Wie in Abbildung 3.15b erkennbar, ist das Auflösungsvermögen erneut entlang aller Richtungen gleich gut.

Um den Kontrastübertrag verschiedener Raumfrequenzen (Strukturgrößen) von der Objekt- in die Detektorebene abzuschätzen, bietet sich die Abbildung einer der Zonenplatten $\left(\mathrm{ZP}_{2}\right)$ an. Das Bildfeld beträgt bei einer 375fach vergrößerten Aufnahme mit $\mathrm{ZP}_{3}$ allerdings nur etwa $35 \mu \mathrm{m}$. ZP2 (Durchmesser $190 \mu \mathrm{m}$ ) kann demnach mit einer Aufnahme nicht komplett abgebildet werden. Daher wurden fünf Einzelbilder der Zonenplatte aufgenommen $(t=30 \mathrm{~min}$, Ellipsoidposition $\mathrm{B}, \mathrm{ZP} 3)$, die entsprechend in horizontaler Richtung zueinander versetzt sind. Abbildung 3.16 zeigt das zusammengesetzte Mikroskopbild. Das Profil wurde entlang der gezeigten Markierung ermittelt, wobei über knapp 30 Pixel (etwa $1 \mu \mathrm{m}$ Breite) gemittelt wurde. Für verschiedene Raumfrequenzen wurde aus dem Profil jeweils das Intensitätsmaximum und -minimum ermittel und daraus nach Gleichung (3.5) der Kontrast als Funktion der 

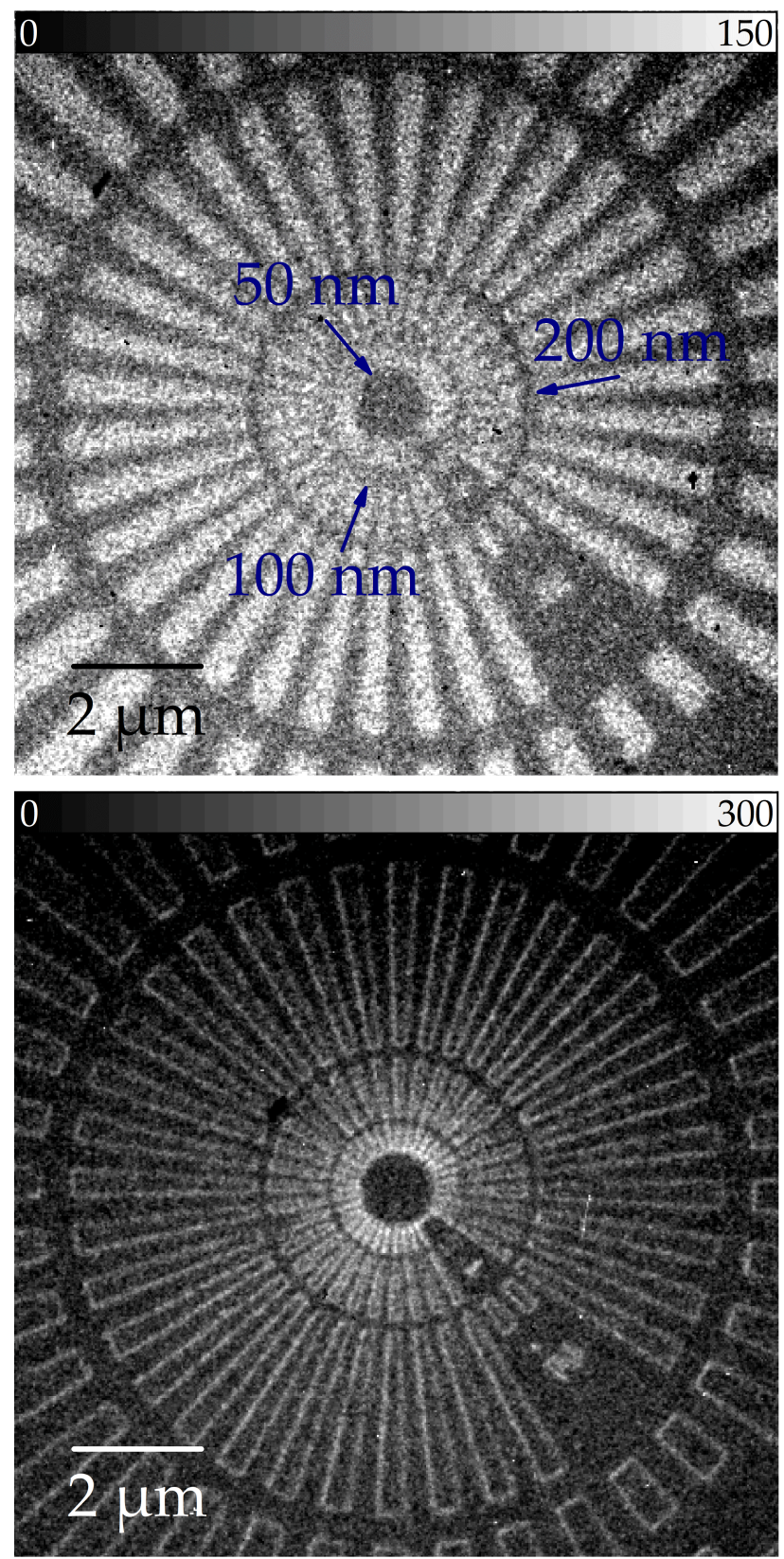

Abbildung 3.14: Röntgenmikroskopische Aufnahme eines Siemenssterns ( $M=$ $470, t=120 \mathrm{~min}$, NTT-AT) im Hellfeld- (oben) und Dunkelfeldkontrast (unten). 


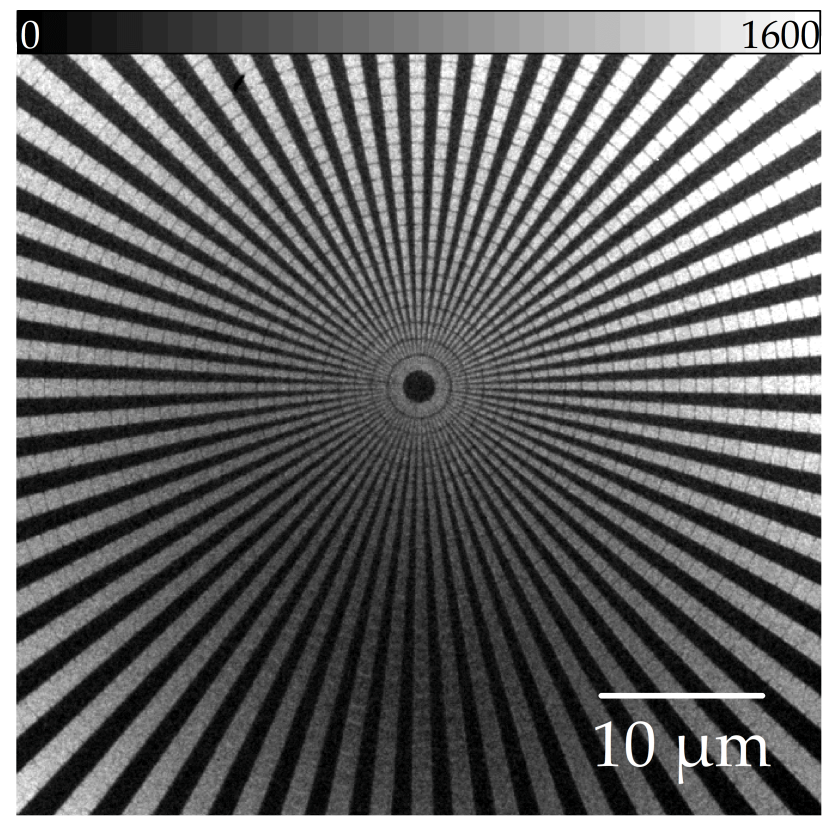

(a) $M=175, t=5 \mathrm{~min}$

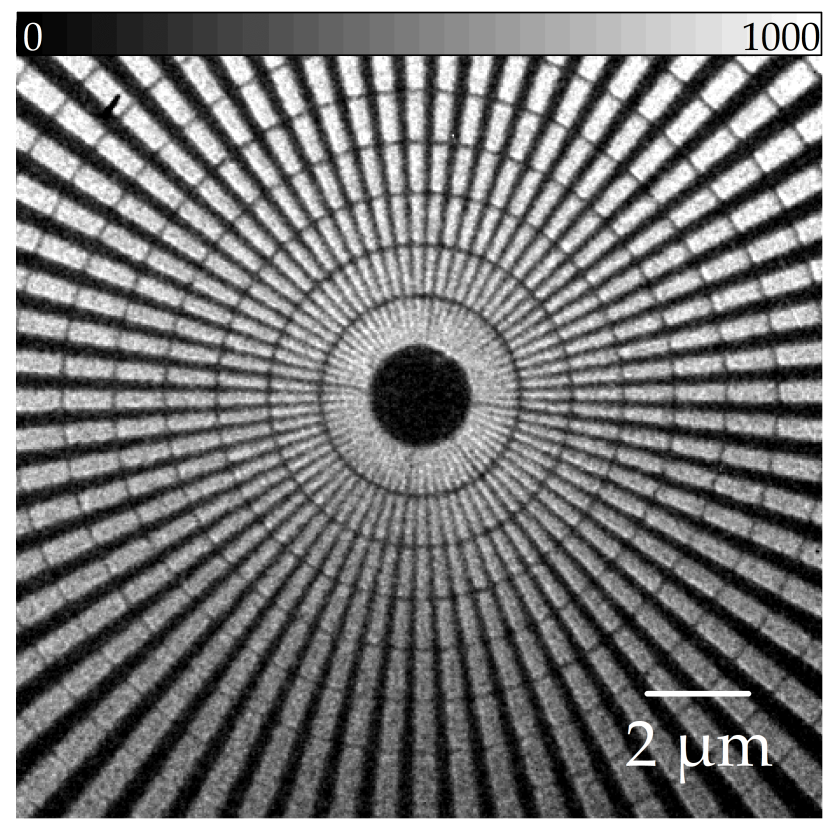

(b) $M=375, t=30 \mathrm{~min}$

Abbildung 3.15: Röntgenmikroskopische Aufnahmen eines Siemenssterns (ZonePlate Ltd) für verschiedene Vergrößerungen und Belichtungszeiten 

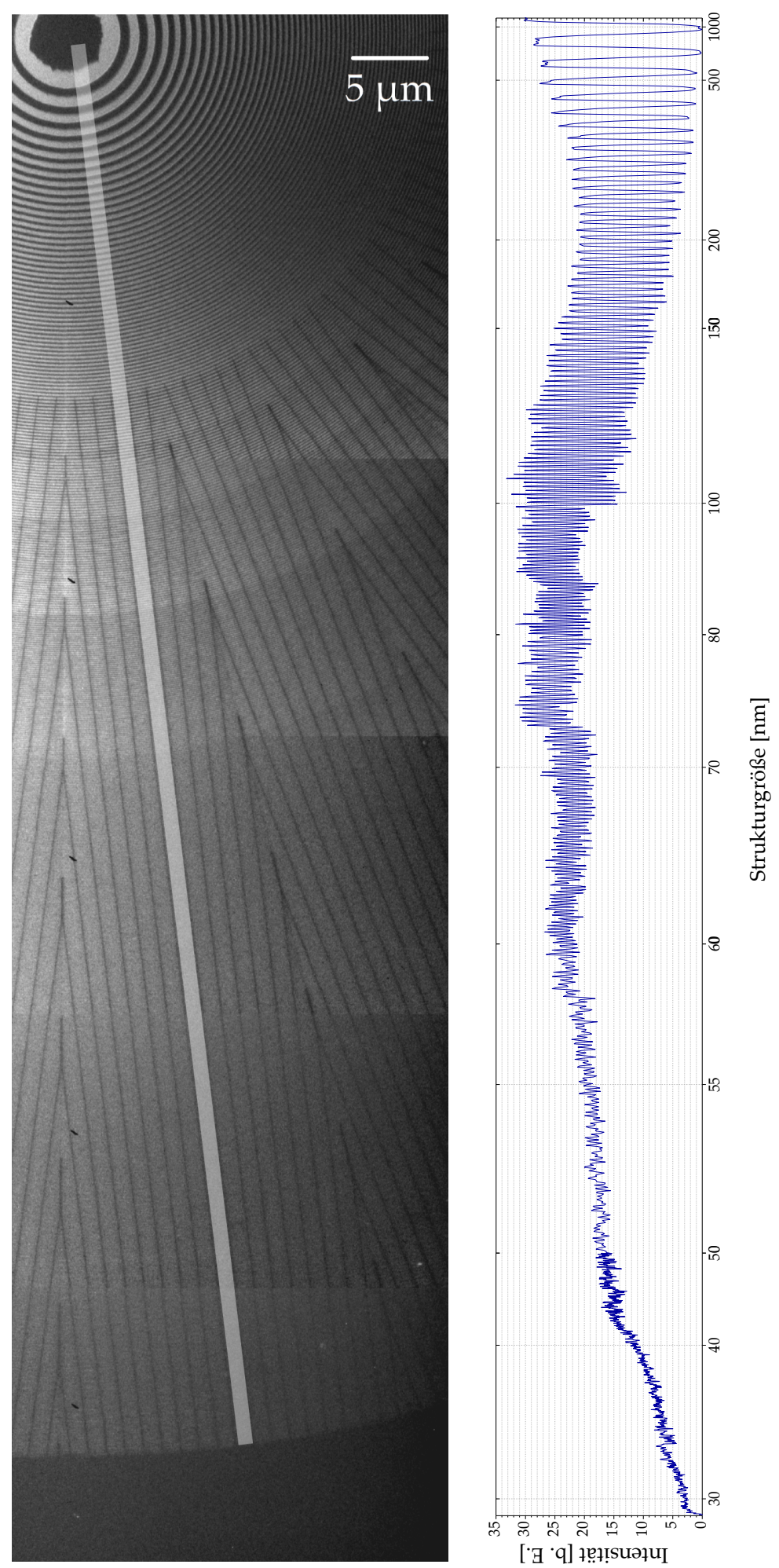

Abbildung 3.16: Röntgenmikroskopische Aufnahme der Zonenplatte ZP2 ( $M=$ $375, \mathrm{t}=30 \mathrm{~min}$ ) zusammengesetzt aus fünf Einzelbildern (links) und Profil (rechts) ermittelt entlang der weißen Markierung 


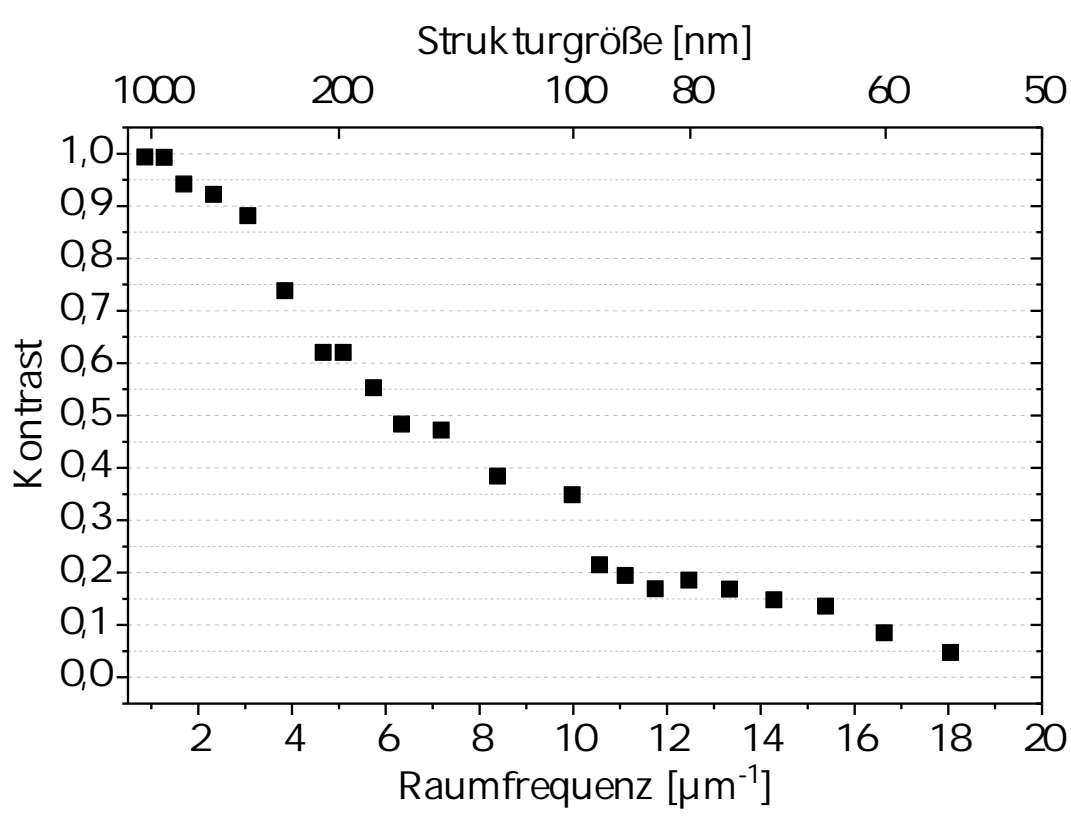

Abbildung 3.17: Aus dem Profil der Zonenplatte ZP2 berechneter Kontrast als Funktion der Raumfrequenz bzw. Strukturgröße

Raumfrequenz berechnet (siehe Abb. 3.17). Strukturen bis zu einer GröBe von ungefähr $60 \mathrm{~nm}$ bzw. Raumfrequenzen bis etwa $16,7 \mu \mathrm{m}^{-1}$ können demnach räumlich aufgelöst werden.

Das Auflösungsvermögen ist jedoch nicht durch die verwendeten Zonenplatten, sondern durch die effektive Pixelgröße der Kamera und das geringe Signal-Rausch-Verhältnis (SNR) begrenzt. Um die Auflösung zu verbessern, müssten die Objekte daher bei einer stärkeren Vergrößerung abgebildet werden. Gleichzeitig muss die Brillanz der Quelle gesteigert werden, damit für ein hinreichend hohes SNR genügend Photonen detektiert werden können (siehe Abs. 3.3.5).

\subsubsection{BIOLOGISCHE UND GEOLOGISCHE PROBEN}

Nach der Charakterisierung des Laborröntgenmikroskops wurden unterschiedliche biologische und geologische Proben untersucht. Eine Zusammenstellung der Mikroskopbilder zeigt Abbildung 3.18. Alle Proben wurden in wässriger Lösung auf eine $100 \mathrm{~nm}$ dicke $\mathrm{Si}_{3} \mathrm{~N}_{4}$-Membran (Transmission $T \approx 0,5$ bei $\lambda=2,88 \mathrm{~nm}$ ) aufgebracht und eingetrocknet.

Als Erstes wurde das extremophile Bakterium Deinococcus Radiodurans abgebildet. Es zeichnet sich durch eine extrem hohe Resistenz gegenüber ionisierender Strahlung auch in hohen Dosen aus. Das D. Radiodurans kann zudem unter Vakuumbedingungen überleben und ist somit ein ideales Objekt für die Röntgenmikroskopie. Typischerweise besteht das Bakterium aus vier Zellen, die sich in Form eines vierblättrigen Kleeblatts anordnen. Diese charakteristische Struktur der Deinococcus Radi- 


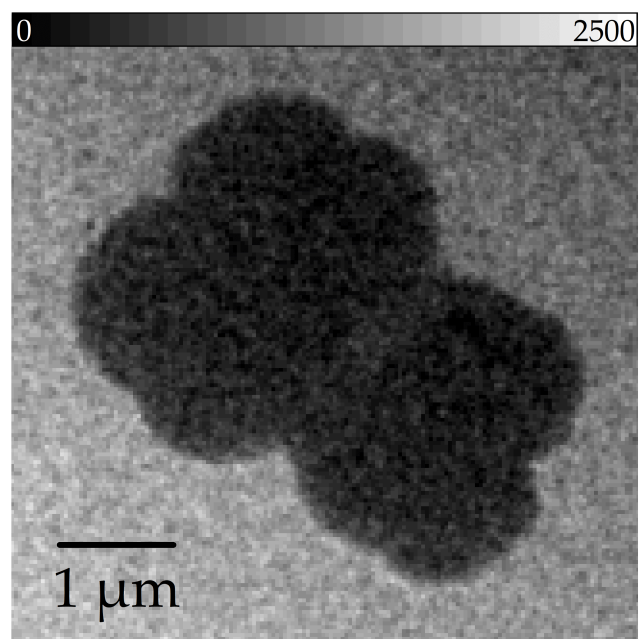

(a) $M=375, t=30 \mathrm{~min}$

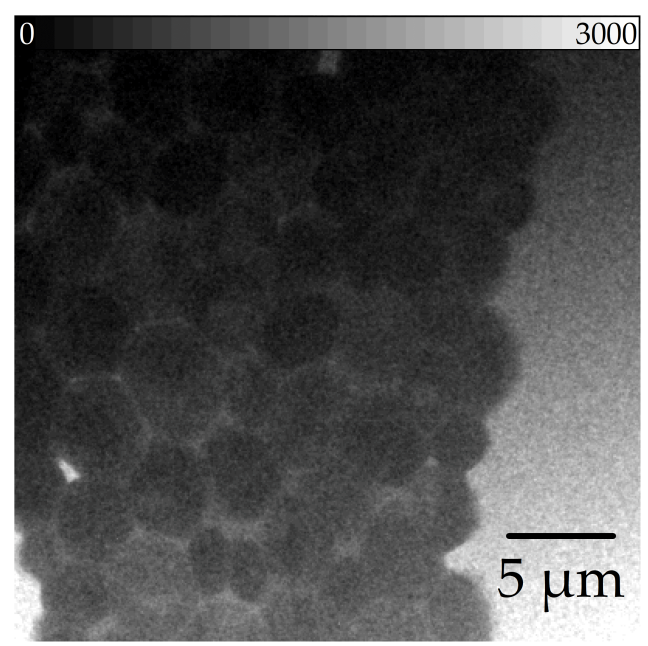

(c) $M=175, t=5 \mathrm{~min}$

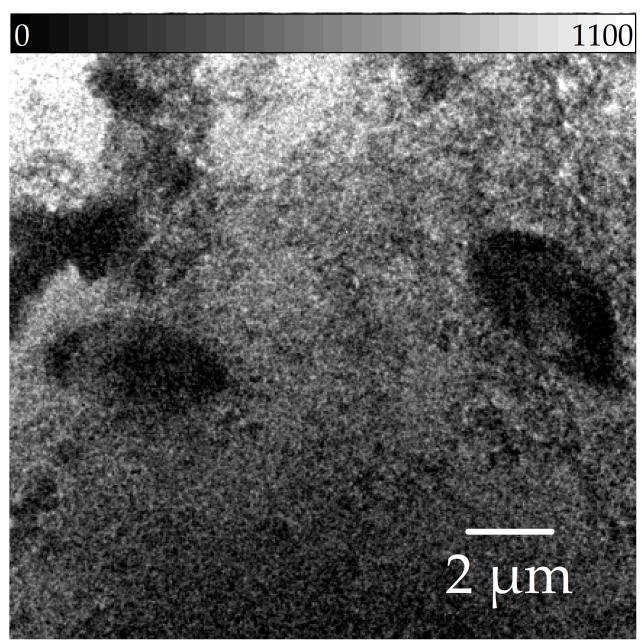

(b) $M=375, t=30 \mathrm{~min}$

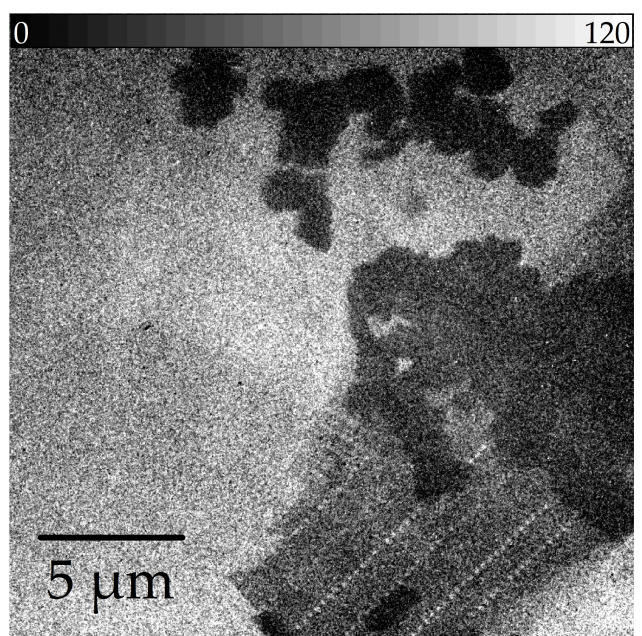

(d) $M=500, t=60 \mathrm{~min}$

Abbildung 3.18: Röntgenmikroskopische Aufnahmen von (a) Deinococcus Radiodurans (DSM-Nr. 20 539), (b) Mikroorganismen, (c) Hefezellen und (d) GeoKolloiden. Die D. Radiodurans wurden von Marius Priebe und Tim Salditt (Univ. Göttingen), die Mikroorganismen sowie Kolloide von Jürgen Niemeyer (Univ. Göttingen) und die Hefezellen von René Siegmund (LLG) bereitgestellt. 
odurans ist in Abbildung 3.18a eindeutig zu erkennen. Die innere Struktur ist hingegen nicht sichtbar, da die weiche Röntgenstrahlung vollständig vom Objekt absorbiert wird.

In Abbildung 3.18b sind zwei Bakterien zu sehen, die aus dem Biofilm einer im wässrigen System korrodierenden Stahlprobe entnommen wurden. Die Mikroorganismen heben sich deutlich von anderem organischen und anorganischen Debrismaterial der Probe ab. Beide Bakterien weisen zudem einen kleinen Bereich geringerer Absorption auf. Die Mikroorganismen könnten - bis auf diesen Bereich - mit anderen Elementen wie Eisen oder Mangan angereichert sein. Dadurch erhöht sich die Absorption weicher Röntgenstrahlung. Ebenfalls möglich erscheint, dass die Mikroorganismen durch das Vakuum dehydriert wurden und ihre Struktur eingebüßt haben. An diesen Stellen sind die Proben daher sehr viel dünner, sodass weniger Photonen absorbiert werden.

Als drittes biologisches Objekt wurden Hefezellen abgebildet (siehe Abb. 3.18c). Ähnlich wie bei den Deinococcus Radiodurans ist auch bei den Hefezellen nur die äußere Form, aber keine innere Struktur sichtbar.

Zusätzlich zu den biologischen Proben wurden Geo-Kolloide aus dem Main untersucht (siehe Abb. 3.18d). Zu sehen sind sowohl mikro-strukturierte als auch unregelmäßige Strukturen. Das Objekt mit der perlschnurartigen Struktur im unteren Teil des Mikroskopbildes ist sehr wahrscheinlich ein Fragment der Hülle einer Kieselalge. Neben der typischen Struktur von Diatomeen bestätigen EDX-Messungen (engl. energy dispersive $\mathrm{x}$-ray spectroscopy) mit einem Rasterelektronenmikroskop diese Vermutung: Das Objekt besteht aus Siliciumdioxid $\left(\mathrm{SiO}_{2}\right)$, das charakteristisch für die Hülle von Kieselalgen ist. Die isolierten „Löcher" dieser Struktur haben einen Abstand von etwa $215 \mathrm{~nm}$. Die unregelmäßig strukturierten Objekte sind hingegen Kolloide, die aus Calciumcarbonat $\left(\mathrm{CaCO}_{3}\right)$ bestehen.

Abschließend wurden drei verschiedenen Algen des Genus Trachelomonas untersucht. Diese einzelligen, eukaryotischen Lebewesen besitzen einen peitschenähnlichen Zellfortsatz (Flagellum) und sind von einer starren, mit Dornen besetzten Hülle (Lorica) umgeben. Die Lorica beinhaltet zudem Mangan- und Eisenverbindungen. Im Lichtmikroskop sind die Zellinhalte wie der Zellkern oder die Chloroplasten daher oft nicht sichtbar. Die drei Algen wurden deshalb auch mit dem Laborröntgenmikroskop abgebildet (siehe Abb. 3.19). Im Gegensatz zu dem Bakterium D. Radiodurans oder den Hefezellen durchdringt die weiche Röntgenstrahlung die Zellen der Algen zumindest teilweise. Dennoch sind die Zellbestandteile nicht zu erkennen. Umso deutlicher sind dafür die charakteristischen Dornen der elliptischen Lorica und das Flagellum in den röntgenmikroskopischen Aufnahmen zu sehen.

Insgesamt zeigt sich, dass die Abbildung biologischer Objekte mit dem Laborröntgenmikroskop bisher nur eingeschränkt möglich ist. Vor 

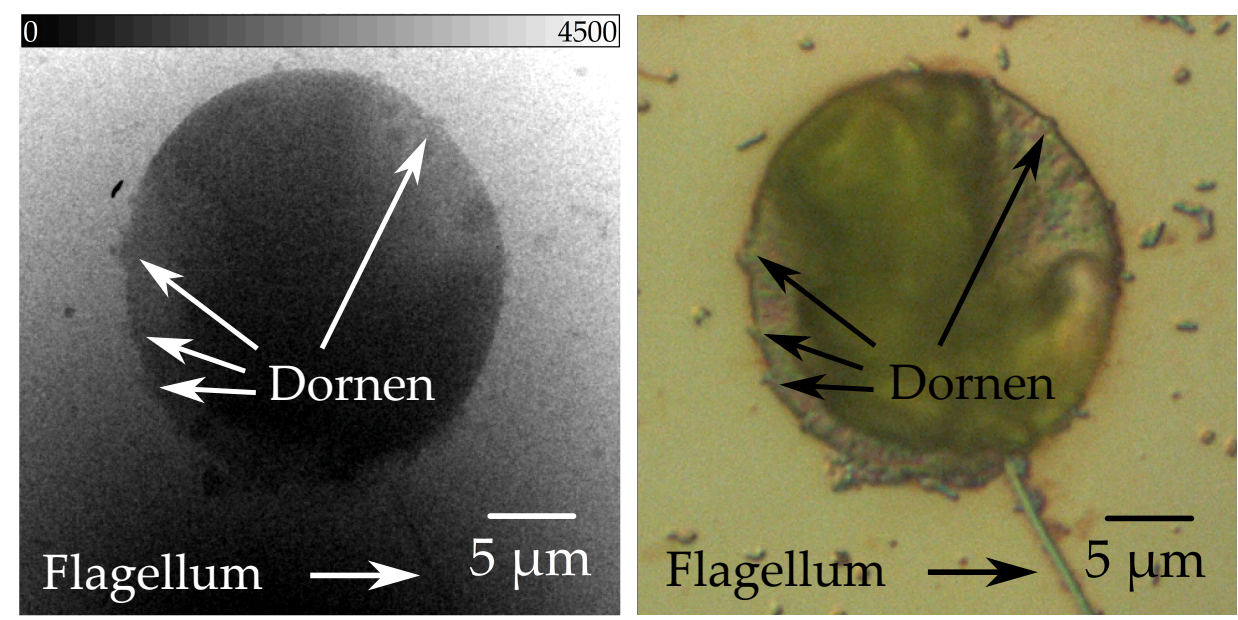

(a) Trachelomonas Levefrei (SAG 1283-10), $M=175, \mathrm{t}=5 \mathrm{~min}$
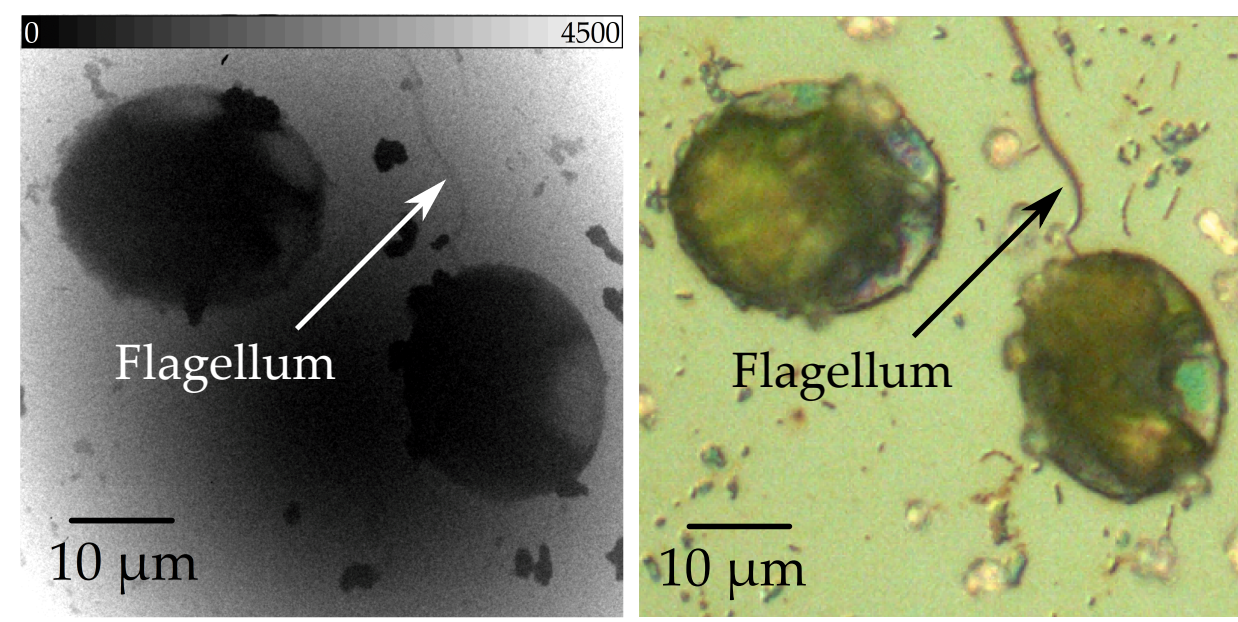

(b) Trachelomonas Hispida (SAG 1283-1), $M=175, \mathrm{t}=5 \mathrm{~min}$
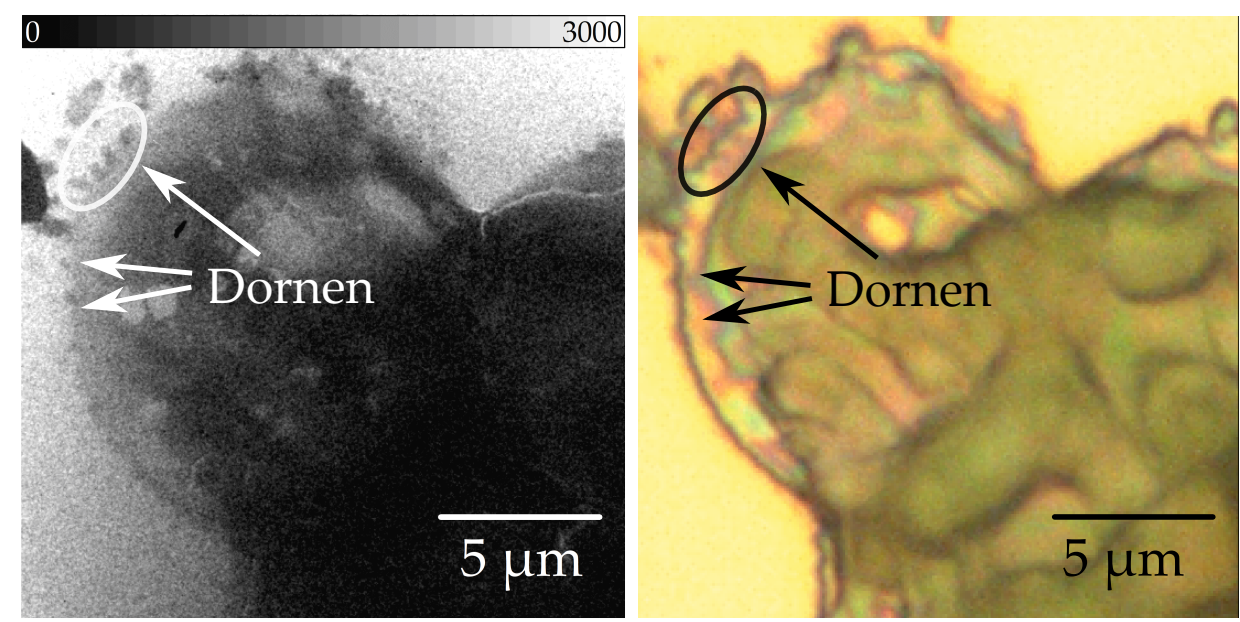

(c) Trachelomonas Oblonga (SAG 1283-11), $M=375, t=60 \mathrm{~min}$

Abbildung 3.19: Röntgen- (links) und lichtmikroskopische (rechts) Aufnahmen verschiedener Algen, bereit gestellt von Maike Lorenz (Sammlung von Algenkulturen Göttingen). Die Algen wurden mit dem Lichtmikroskop $(M=100)$ im differenziellen Interferenzkontrast (Nomarski-Kontrast) abgebildet. 
allem die geringe Brillanz der Quelle limitiert das Signal-Rausch-Verhältnis und damit den Kontrast der Mikroskopaufnahmen. Um zukünftig auch die innere Struktur von Proben untersuchen zu können, werden daher in Abschnitt 3.3.5 einige Maßnahmen zur Optimierung des Mikroskops vorgestellt.

\subsubsection{KOHÄRENZANALYSE}

Alternativ zur „klassischen“ Röntgenmikroskopie werden „linsenlose“ Abbildungsverfahren verwendet (siehe Abs. 3.1.4). Dadurch wird das Auflösungsvermögen nicht durch die abbildende Optik begrenzt und zudem werden Abbildungsfehler vermieden. Für kompakte Laborquellen ist der Verzicht auf die abbildende Optik zusätzlich von Vorteil, da mehr Photonen für die Abbildung genutzt werden, die sonst aufgrund der geringen Effizienz der Optik (z. B. Zonenplatte siehe Abb. 3.6) verloren gingen. Eine Voraussetzung für diese Methoden ist jedoch, dass die Objekte mit kohärenter Röntgenstrahlung beleuchtet werden. Laserinduzierte Plasmen weisen allerdings per se eine geringe Kohärenz auf. Um zu beurteilen, ob die in dieser Arbeit verwendete Plasmaquelle dennoch für die kohärente Bildgebung geeignet sein könnte, wurde der Kohärenzgrad $\gamma$ der Quelle sowohl aus der Intensitätsverteilung des Plasmas nach Van-Cittert-Zernike-Theorem (siehe Abs. 3.1.3) als auch aus dem Beugungsbild einer $10 \mu \mathrm{m}$-Lochblende ermittelt.

Zur Berechnung des Kohärenzgrades nach Van-Cittert-Zernike wurde mit der Lochkamera die Intensitätsverteilung des Stickstoffplasmas bei $\lambda=2,88 \mathrm{~nm}$ gemessen (siehe Abb. 3.20a) und an das horizontale Intensitätsprofil eine Gaußfunktion $\mathrm{I}(\mathrm{u})$ angepasst (siehe Abb. 3.20b). Aus dieser wurde nach Gleichung (3.29) der Kohärenzgrad $\gamma(x)$ für den Abstand $z=1135 \mathrm{~mm}$ zur Quelle berechnet, der der Objektebene des Beugungsexperiments entspricht. Das Ergebnis ist in Abbildung 3.21 aufgetragen: Die Kohärenzlänge (Abfall Kohärenzgrad auf $1 / e \approx 0,37$ ) der Plasmaquelle beträgt für diesen Abstand $l_{c}^{\text {ohne }}=3,8 \mu \mathrm{m}$ und reicht demnach nicht aus, um das Beugungsbild der $10 \mu \mathrm{m}$-Lochblende aufzunehmen. Um den Kohärenzgrad ausreichend zu erhöhen, müsste der Abstand zwischen Quelle und Objekt auf etwa 3000 mm vergrößert werden. Da die kompakte Bauweise des Aufbaus erhalten bleiben soll, wird stattdessen zusätzlich eine Blende (Durchmesser $100 \mu \mathrm{m}$ ) nahe des Plasmas (Abstand zur Quelle $40 \mathrm{~mm}$ ) angebracht und so die Plasmagröße verringert. Die Intensitätsverteilung mit Quellapertur lässt sich durch eine schmalere Gaußkurve annähern (siehe Abb. 3.2ob) und der wiederum nach Van-Cittert-Zernike berechnete Kohärenzgrad erhöht sich. Die Kohärenzlänge nimmt etwa um den Faktor 3,5 auf $l_{\mathrm{c}}^{\mathrm{mit}}=13,2 \mu \mathrm{m} \mathrm{zu}$. Das Beugungsbild der Lochblende sollte unter Verwendung der Aper- 


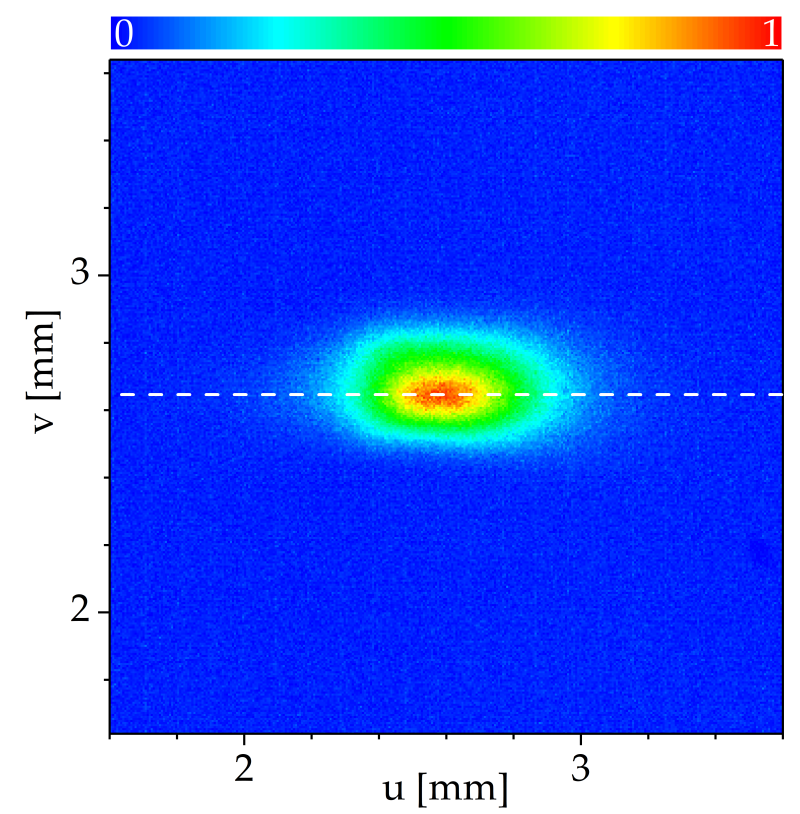

(a) Intensitätsverteilung (Mittelung 100 Pulse)

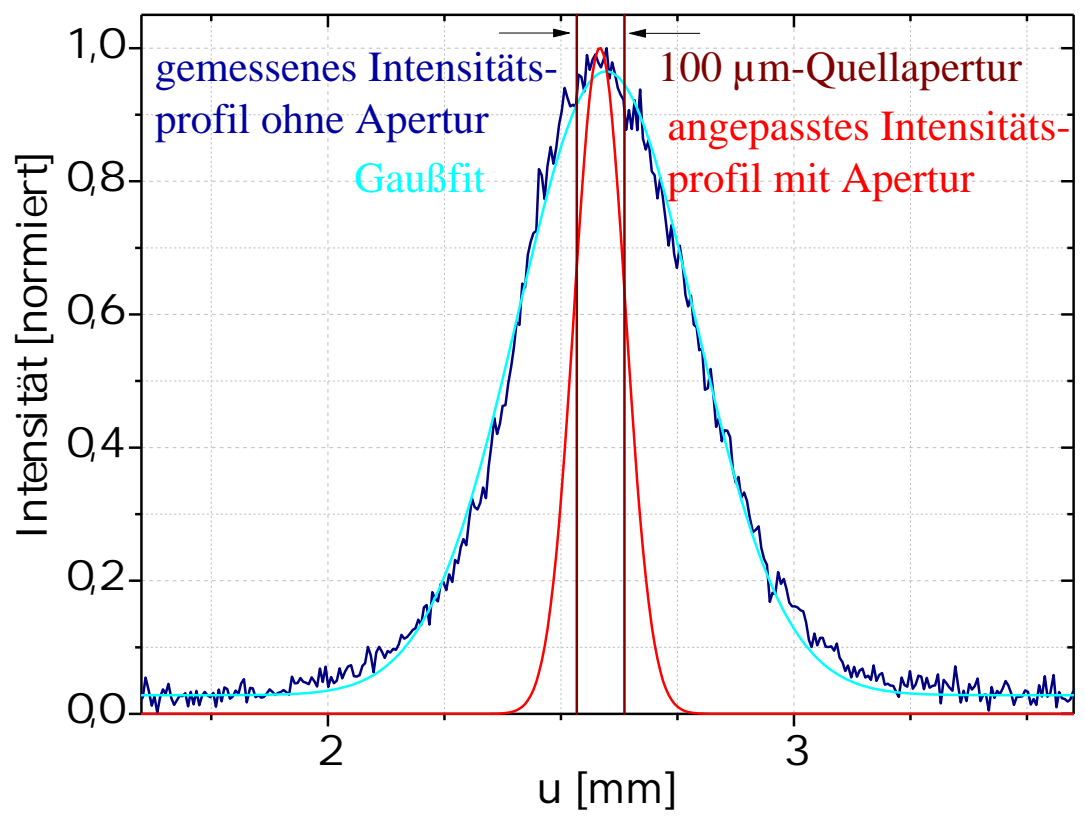

(b) Horizontale Intensitätsprofile und Gaußfits

Abbildung 3.20: (a) Lochkameraaufnahme (ohne Quellapertur) und (b) Intensitätsprofil des Stickstoffplasmas bei $\lambda=2,88 \mathrm{~nm}$ mit angepasster Gaußfunktion sowie angepasste Gaußfunktion zur Näherung des Intensitätsprofil bei Verwendung einer $100 \mu \mathrm{m}$-Quellapertur (Fitparameter siehe Tab. A.4). 


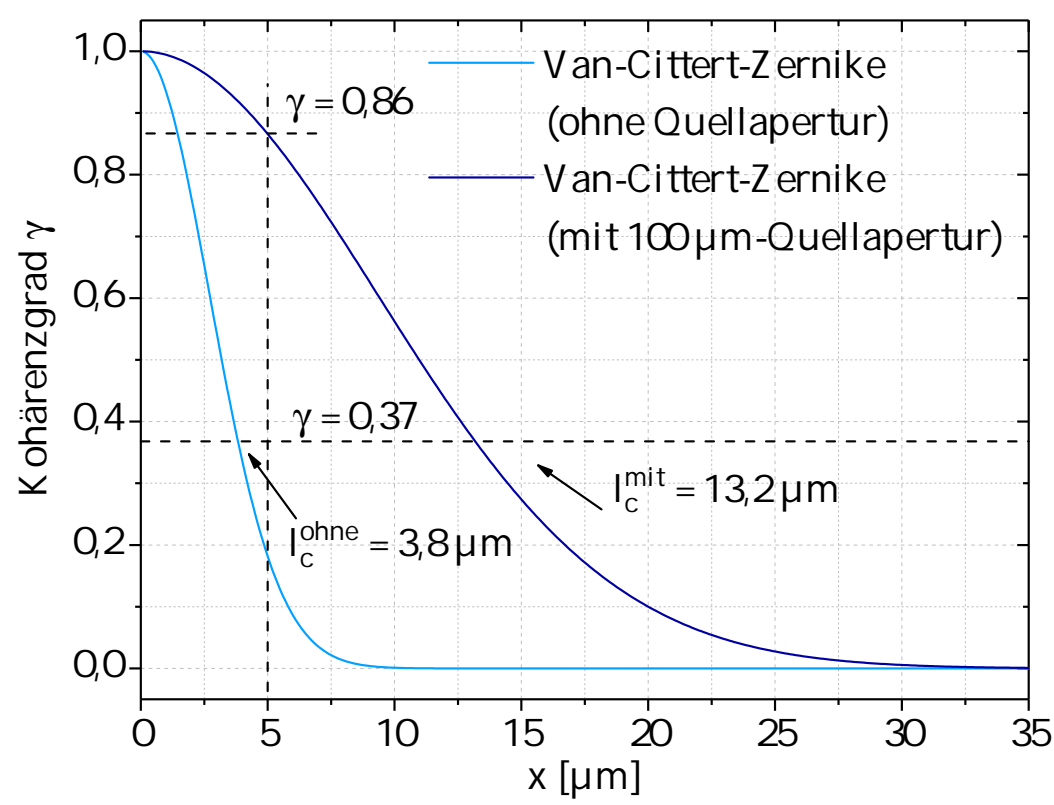

Abbildung 3.21: Kohärenzgrad $\gamma$ der Plasmaquelle ohne und mit einer $100 \mu \mathrm{m}-$ Quellapertur berechnet nach Van-Cittert-Zernike-Theorem

tur also messbar sein. Für den Blendenradius von $5 \mu \mathrm{m}$ beträgt der Kohärenzgrad etwa $\gamma^{5 \mu m} \approx 0,86$.

Der Aufbau für die Aufnahme des Beugungsbildes der Lochblende ist zusammen mit der gemessenen Intensitätsverteilung in Abbildung 3.22 gezeigt. Der Abstand zwischen Quelle und Objekt beträgt $z=1135 \mathrm{~mm}$. Die Kamera (Roper Scientific, 1024 x 1024 Pixel, Pixelgröße $13 \mu \mathrm{m}$ ) befindet sich $215 \mathrm{~mm}$ dahinter. Die Beugungsringe der Blende sind $\mathrm{zu}$ erkennen. In der kohärenten Bildgebung (z. B. CDI) würde aus diesem Beugungsbild das (unbekannte) Objekt rekonstruiert. Hier wird aus dieser Aufnahme stattdessen der Kohärenzgrad der Quelle bestimmt. Dazu
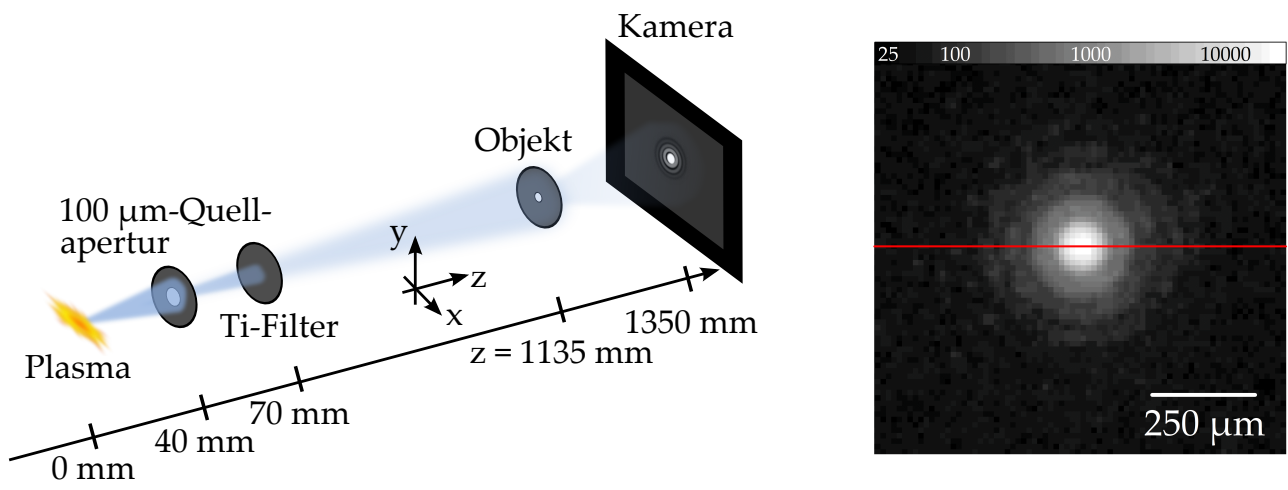

Abbildung 3.22: Schematische Darstellung des Aufbaus zur kohärenten Bildgebung (links) und Beugungsbild der $10 \mu \mathrm{m}$-Lochblende (rechts, $t=60 \mathrm{~min}$ ) 
wurde an den Querschnitt durch das Beugungsbild (siehe Abb. 3.23) die Funktion

$$
\mathrm{F}(\mathrm{x})=\underbrace{\left[\mathrm{A} \cdot\left(\frac{\mathrm{J}_{1}\left(\pi\left(x-x_{\mathrm{c}}\right) \frac{\mathrm{d}}{\lambda z}\right)}{\pi\left(x-x_{\mathrm{c}}\right) \frac{\mathrm{d}}{\lambda z}}\right)^{2}\right]}_{\text {Intensitätsverteilung Beugung an Lochblende }} * \underbrace{\left[\mathrm{c}+\mathrm{B} \cdot \mathrm{e}^{\left.-\mathrm{m} x^{2}\right]}\right.}_{\text {Intensitätsverteilung Plasmaquelle }}
$$

angepasst (Parameter siehe Tab. A.5), wobei $\mathrm{J}_{1}$ die Besselfunktion erster Art bezeichnet. Die charakteristische Intensitätsverteilung nach Beugung an einer Lochblende wird dabei mit einer Gaußfunktion gefaltet, die die endliche Ausdehnung der Plasmaquelle berücksichtigt und daher $\mathrm{zu}$ einem verringerten Kohärenzgrad führt. Zum Vergleich ist in Abbildung 3.23 das Beugungsbild eingezeichnet, das sich ergeben würde, wenn die Lochblende mit vollständig kohärenter Röntgenstrahlung $(\gamma=1)$ abgebildet werden würde. Der Unterschied ist deutlich sichtbar: Im Gegensatz zur kohärenten Beleuchtung der Lochblende ist die Intensitätverteilung im Fall partiell kohärenter Strahlung weniger stark moduliert. Die Abstände der lokalen Maxima und deren Breite bleiben jedoch unverändert.

Die Gaußfunktion aus Gleichung (3.30) repräsentiert den Einfluss der Intensitätsverteilung des Plasmas auf das Beugungsbild der Lochblende. Daher wird diese zur Berechnung des Kohärenzgrades gemäß VanCittert-Zernike-Theorem genutzt. Die auf diese Art ermittelte Kohärenz-

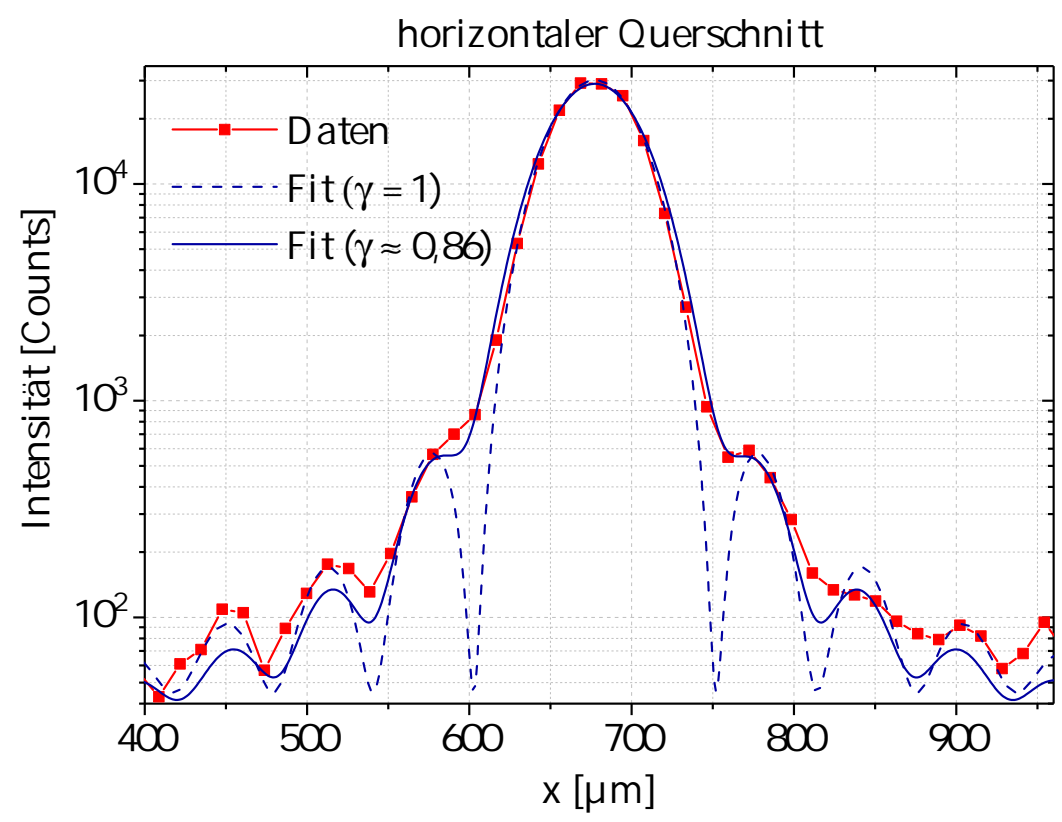

Abbildung 3.23: Horizontaler Querschnitt durch das Beugungsbild der $10 \mu \mathrm{m}-$ Lochblende sowie Fitfunktionen für vollständige $(\gamma=1)$ und partielle Kohärenz $\left(\gamma^{5 \mu \mathrm{m}} \approx 0,86\right.$, Fitparameter siehe Tab. A.5) 
länge von 13,3 $\mu \mathrm{m}$ (siehe Abb. 3.24) stimmt sehr gut mit dem Wert überein, der sich aus der direkt mit Lochkamera gemessenen Intensitätsverteilung des Plasmas mit $100 \mu \mathrm{m}$-Quellapertur ergibt. Beide Methoden sind demnach geeignet, um die Kohärenz der Quelle zu bestimmen. Das Plasmaprofil heranzuziehen, ist jedoch weniger aufwendig.

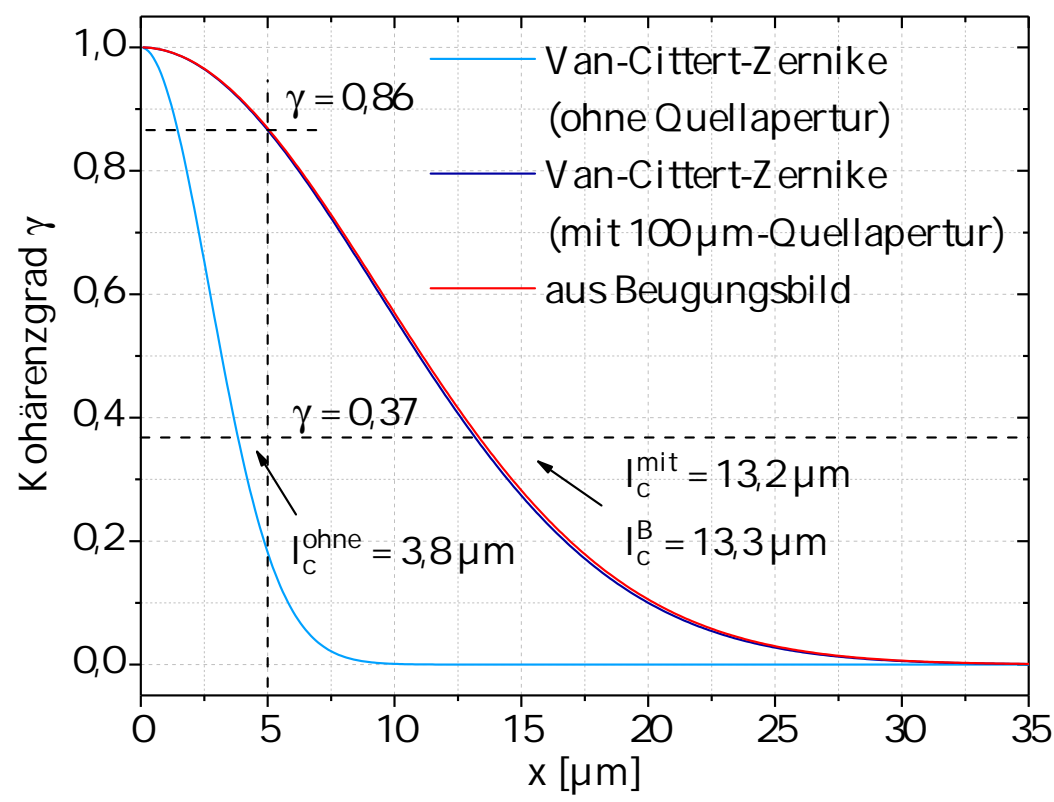

Abbildung 3.24: Kohärenzgrad der Plasmaquelle berechnet nach Van-CittertZernike-Theorem und ermittelt aus Beugungsbild einer $10 \mu \mathrm{m}$-Lochblende

\subsubsection{WEITERENTWICKLUNG DES RÖNTGENMIKROSKOPS}

Wie in Abschnitt 3.3.2 beschrieben, eignet sich das kompakte Röntgenmikroskop sehr gut, um rein absorbierende Objekte mit einer hohen räumlichen Auflösung von 50 bis $60 \mathrm{~nm}$ abzubilden. Die Belichtungszeit liegt dabei für eine Hellfeldaufnahme im Bereich von etwa 30 min. Im Dunkelfeldkontrast sind bis zu 120 min notwendig, um ein ausreichendes Signal-Rausch-Verhältnis zu erhalten. Die Abbildung biologischer und geologischer Proben (siehe Abs. 3.3.3) ist deutlich schwieriger. Insbesondere konnte bisher nur die Form der Objekte, aber nicht deren innere Struktur dargestellt werden.

Um das Signal-Rausch-Verhältnis zu erhöhen, gibt es verschiedene Möglichkeiten, die für ein optimiertes Röntgenmikroskop berücksichtigt werden sollten:

1. Das Plasma sollte wie in Abschnitt 2.3.4 dargestellt unter $30^{\circ}$ statt unter $90^{\circ}$ genutzt werden. Zusätzlich zur höheren Intensität und verbesserten Lagestabilität des Plasmas verringert sich dadurch die Plasmafläche und somit der Fokusdurchmesser in der Objektebene. 
Insgesamt werden auf diese Art mehr Photonen von der Zonenplatte aufgesammelt und tragen zur Abbildung des Objekts bei.

2. Für die Aufnahmen der Testobjekte sowie der biologischen und geologischen Proben wurde der Nanosekundenlaser verwendet, um das Plasma zu zünden. Es konnte jedoch bereits gezeigt werden (siehe Abs. 2.3.4), dass sich die Brillanz der Quelle bei $\lambda=$ 2,88 nm mit einem Pikosekundenlaser etwa um eine Größenordnung steigern lässt [81]. Um den Pikosekundenlaser auch im Röntgenmikroskop zu verwenden, muss allerdings erst die in Abschnitt 2.3.4 beschriebene Problematik der verstärkten Debrisproduktion durch Ablation des Düsenplättchens insbesondere bei hohen Gasdrücken gelöst werden.

3. Nicht nur die Plasmaquelle, auch die eingesetzten Optiken bieten Verbesserungspotenzial. So ist z. B. die Effizienz einer Zonenplatte aus Nickel statt aus Wolfram etwa doppelt so hoch (siehe Abb. 3.6). Der Kondensor könnte hinsichtlich seiner Geometrie angepasst werden. Ein geringerer Abstand zwischen den Brennpunkten des Ellipsoids ermöglicht eine noch kompaktere Bauweise des Mikroskops. Darüber hinaus könnten mehr Photonen vom Plasma aufgesammelt werden, wenn die Eingangsapertur des Spiegels größer wäre. Bei einem kürzeren Spiegel und damit einer kleineren Spiegeloberfläche ließe sich außerdem die vom Hersteller angegebene Rauheit von 0,5 nm eher über die gesamte Fläche erreichen. Dadurch könnte die Reflektivität von derzeit 30\% auf vermutlich über $50 \%$ erhöht werden.

4. Das Aufbringen wässriger Lösungen auf eine $\mathrm{Si}_{3} \mathrm{~N}_{4}$-Membran ist nur bedingt geeignet, um hinreichend dünne Proben hoher Transmission zu erhalten. Wenn möglich, könnten die Objekte - ähnlich zur Transmissionselektronenmikroskopie - eingebettet und mit einem Mikrotom geschnitten werden. Diese Präparationsmethode schließt zwar u.a. die Untersuchung ganzer Zellen aus, dennoch ließen sich auch aus einzelnen Probenschnitten interessante Informationen über die innere Struktur des Objekts gewinnen. Zugleich erhöht sich durch die chemische Fixierung die Resistenz der Proben gegenüber Strahlenschäden.

Die Umsetzung dieser Vorschläge erfordert eine zum Teil aufwendige Neukonstruktion des Mikroskops und ist mit zusätzlichen Kosten verbunden. Die Möglichkeiten zur Anwendung des Mikroskops blieben dennoch eingeschränkt. Insbesondere die dreidimensionale Abbildung kryofixierter Zellen, die an synchrotronbasierten Mikroskopen mittlerweile zum Standard gehört, wäre trotz der Verbesserungen wahrscheinlich nicht möglich. Ein höheres Anwendungspotenzial verspricht statt- 
dessen die Kombination aus Röntgenmikroskopie und z. B. STED-Mikroskopie oder NEXAFS-Spektroskopie: Ein STED-Röntgenmikroskop liefert aufgrund der komplementären Kontrastverfahren beider Methoden ein umfassenderes Bild der Probe. Für harte Röntgenstrahlung nutzen Bernhardt et al. diesen korrelativen Mikroskopieansatz am Synchrotron (PETRA III, DESY) bereits zur Abbildung des Aktin-Zytoskeletts in Zellen aus dem Herzgewebe [210]. Die Verknüpfung von Mikroskopie und Spektroskopie erlaubt wegen des elementspezifischen Kontrastes die chemische Zusammensetzung der Probe mit hoher Ortsauflösung zu bestimmen. Inwiefern diese Spektromikroskopie auch mit einer kompakten, laserinduzierten Plasmaquelle umgesetzt werden könnte, wird in Abschnitt 5.2 erläutert. 

Röntgenstrahlung wird nicht nur zur hochauflösenden Bildgebung eingesetzt (siehe Kap. 3), sondern bildet auch die Grundlage für zahlreiche analytische Methoden von Medizin, Chemie, Physik, Biologie und Materialwissenschaften. Bereits seit Beginn des 20. Jahrhunderts wird z. B. zur Strukturanalyse die Röntgenbeugung ${ }^{1}$ verwendet. Seit Anfang der 1970er hat sich ebenso die Röntgenabsorptionsspektroskopie (engl. x-ray absorption spectroscopy, XAS) als Analyseverfahren etabliert [213]. Abhängig von den chemischen Struktureigenschaften (Elektronenkonfiguration) der untersuchten Probe wird dabei die Änderung des Absorptionskoeffizienten $\mu$ als Funktion der Photonenenergie gemessen. Von besonderem Interesse ist dabei der Energiebereich, in dem sich der Absorptionskoeffizient sprunghaft ändert. An diesen Absorptionskanten tritt eine ausgeprägte Feinstruktur auf, die einen chemischen „Fingerabdruck“ der Probe darstellt. Entsprechend wird dieses Verfahren als RöntgenNahkanten-Absorptionsspektroskopie (engl. near-edge x-ray absorption fine structure, NEXAFS) bezeichnet. Die zunehmende Verbreitung dieser Methode ist eng mit der Entwicklung von Synchrotronen und in den letzten Jahren ebenso Laborröntgenquellen verknüpft (siehe Kap. 2). Bevor jedoch auf den aktuellen Stand der Forschung (siehe Abs. 4.3), das in dieser Arbeit verwendete Laborröntgenspektrometer (siehe Abs. 4.4) und die untersuchten Materialsysteme (siehe Abs. 4.5) eingegangen wird, soll zunächst erläutert werden, wie die Feinstruktur an der Absorptionskante entsteht, wie sie gemessen wird, und welche Informationen sich aus dem NEXAFS-Spektrum gewinnen lassen. Die Darstellung folgt dabei den Referenzen [31, 214, 215].

\section{I FEINSTRUKTUR DER ABSORPTIONSKANTE}

Röntgenstrahlung im Energiebereich zwischen 250 bis $1250 \mathrm{eV}$ wird in Materie hauptsächlich infolge des photoelektrischen Effekts absorbiert. Ein Röntgenphoton gibt dabei seine Energie an ein kernnahes Elektron der K-, L- oder M-Schale des entsprechenden chemischen Elementes ab, falls dessen Bindungsenergie kleiner als die Energie der einfallenden Röntgenstrahlung ist. Das Elektron wird daraufhin in einen unbesetzten gebundenen Zustand oder ins Kontinuum angeregt.

1 Die Röntgenbeugung an Kristallen wurde 1912 von Max von Laue experimentell realisiert [21I] und ein Jahr später von William H. Bragg und William L. Bragg erstmals zur Strukturaufklärung an Kristallen eingesetzt [212]. 
Um die Absorption von Röntgenstrahlung in Materie zu erklären, wird der lineare Absorptionskoeffizient $\mu$ betrachtet. Dieser ist ein Maß für die Wahrscheinlichkeit, mit der Röntgenstrahlung absorbiert wird. Die Intensität $I_{0}$ der Strahlung nimmt innerhalb einer Probe gemäß dem Lambert-Beer'schen Gesetz [6]

$$
\mathrm{I}=\mathrm{I}_{0} \mathrm{e}^{-\mu \mathrm{d}}
$$

ab, wobei I die Intensität der transmittierten Strahlung und d die Probendicke repräsentieren (siehe auch Abs. 3.1.1). Das Produkt aus Absorptionskoeffizient und Dicke wird optische Dichte $(\mathrm{OD}=\mu \mathrm{d})$ genannt.

Für weite Energiebereiche lässt sich der Absorptionskoeffizient als glatte Funktion von Photonenenergie E und Ordnungszahl Z beschreiben [3I]: $\mu \propto Z^{4} E^{-3}$. Erreicht die Energie der Röntgenstrahlung allerdings die Bindungsenergie kernnaher Elektronen, steigt die Wahrscheinlichkeit zur Absorption eines Röntgenphotons sprunghaft an. Die energetische Lage dieser Absorptionskanten ist für jedes Element charakteristisch. Damit ist die NEXAFS-Spektroskopie elementspezifisch. Im Bereich von 250 bis $1250 \mathrm{eV}$ sind u. a. die Elemente Kohlenstoff, Stickstoff, Sauerstoff, Mangan und Eisen für die NEXAFS-Spektroskopie zugänglich (weitere Elemente siehe Abb. 4.I).

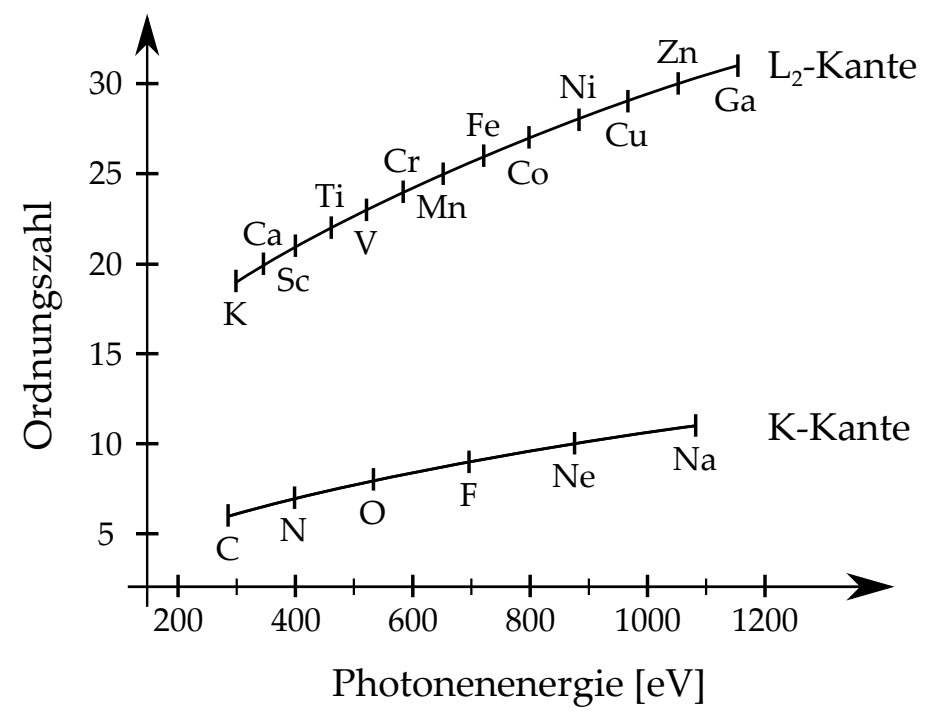

Abbildung 4.1: Übersicht der Elemente, die an der K- bzw. $\mathrm{L}_{2}$-Kante im Energiebereich von 250 bis $1250 \mathrm{eV}$ für die NEXAFS-Spektroskopie zugänglich sind (Daten aus Ref. [216]).

Abhängig von der Umgebung des absorbierenden Atoms weisen die Absorptionskanten außerdem eine Feinstruktur auf. Mit der NEXAFSSpektroskopie wird der Absorptionskoeffizient als Funktion der Energie nahe dieser Absorptionskante untersucht. Strukturen, die im Spektrum einige Zehn bis mehrere Hundert Elektronenvolt oberhalb der 
Kante liegen (Übergänge ins Kontinuum), sind hingegen in der EXAFSSpektroskopie (engl. extended x-ray absorption fine structure) von Interesse [214]. Die Unterteilung des Spektrums folgt dabei aus den zugrundeliegenden physikalischen Prozessen: Die Modulation des Absorptionskoeffizienten im NEXAFS-Bereich wird durch Mehrfachstreuung, im EXAFS-Bereich dagegen durch Einfachstreuung des Elektrons an den Nachbaratomen beschrieben. Das NEXAFS-Signal erlaubt somit Aussagen über die relative Orientierung benachbarter Atome wie Bindungswinkel, Koordination, Oxidationszustände und die Besetzung sowie Hybridisierung von Orbitalen. Die EXAFS-Spektroskopie ist wiederum sensitiv gegenüber Bindungsabständen und Spezies der Nachbaratome. In beiden Fällen wird infolge der Streuung der Elektronen an den Atomen die lokale Struktur der absorbierenden Atome bestimmt. Die Absorptionsröntgenspektroskopie ist daher nicht darauf angewiesen, dass eine Fernordnung wie Kristallstrukturen (Röntgenbeugung), ausgeprägte Dipolmomente (IR-Spektroskopie) oder spezielle funktionelle Gruppen wie chromophore Strukturen (UV-Vis-Spektroskopie) vorhanden sind, und eignet sich daher ebenso zur Untersuchung amorpher Materialien.

Abbildung 4.2 zeigt schematisch das Potenzial eines zweiatomigen Moleküls. Im Unterschied zu einzelnen Atomen entstehen gemeinsame

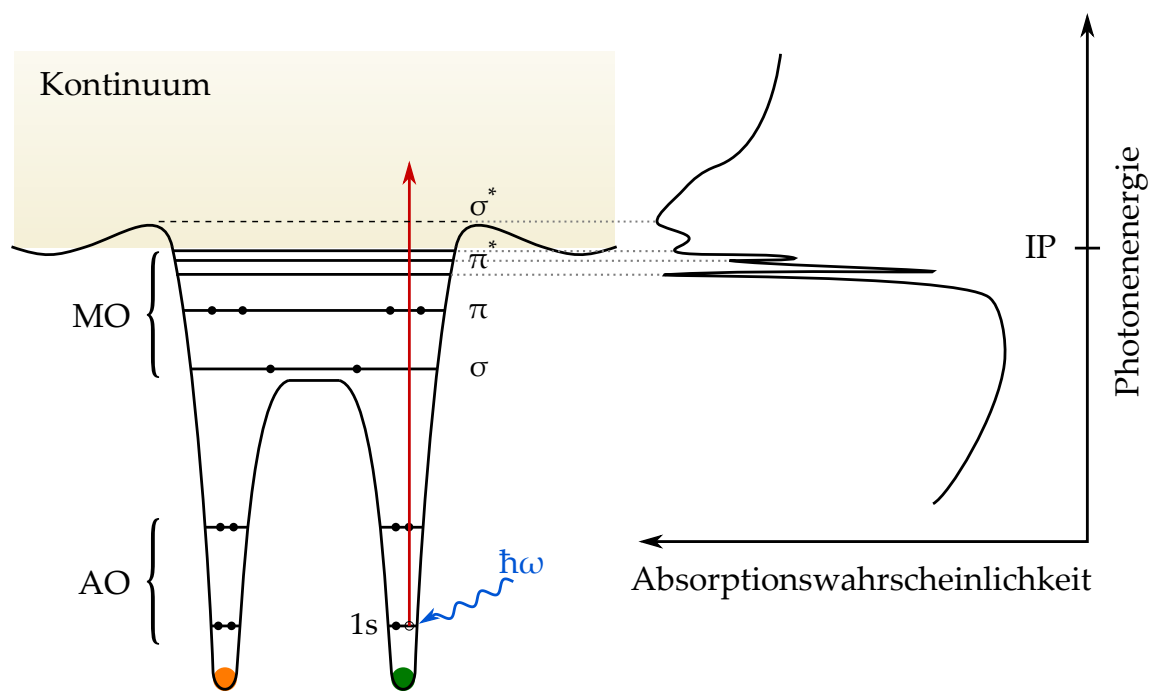

Abbildung 4.2: Schematische Darstellung des Potenzialverlaufs für ein zweiatomiges Molekül (links) und des K-Schalen-Absorptionsspektrums mit charakteristischer Feinstruktur (rechts) nach Referenz [214]

Molekülorbitale (MO), da beide Atomorbitale (AO) an den chemischen Bindungen beteiligt sind. Dabei wird zwischen bindenden und antibindenden $\sigma$ - und $\pi$-Orbitalen unterschieden, wobei unbesetzte Molekülorbitale mit einem Stern (z. B. $\sigma^{*}$ ) gekennzeichnet sind. Die spezifische Feinstruktur im entsprechenden K-Schalen-Absorptionsspektrum (siehe Abb. 4.2) ergibt sich aus den verschiedenen Übergängen kernnaher Elektronen infolge der Absorption der Röntgenstrahlung: Die einzelnen 
Peaks unterhalb des Ionisationspotenzials (IP) bilden die Anregung in unbesetzte $\pi^{*}$-Orbitale ab. Übergänge in unbesetzte $\sigma^{*}$-Orbitale führen hingegen zu breiten Banden oberhalb des Ionisationspotenzials.

Die Absorption von Röntgenstrahlung durch Anregung kernnaher Elektronen ist nur möglich, wenn für das Elektron unbesetzte Zustände zur Verfügung stehen. Diese müssen sowohl die richtige Energiedifferenz als auch den passenden Gesamtdrehimpuls aufweisen. Für elektronische Übergänge müssen dazu die Dipolauswahlregeln beachtet werden. Da bei der Absorption eines Röntgenphotons der Drehimpuls erhalten bleiben muss, gilt für die Änderung des Bahndrehimpulses $\Delta \mathrm{l}= \pm 1$. Zudem ändert sich die Spinquantenzahl nicht $(\Delta s=0)$, sodass die Änderung des Gesamtdrehimpulses $\Delta \mathrm{J}=0, \pm 1$ beträgt. Folglich können die Elektronen aus dem s-Orbital an der $\mathrm{K}$ - und $\mathrm{L}_{1}$-Kante $(\mathrm{l}=0)$ nur in p-Orbitale $(l=1)$ angeregt werden. Eine Absorption an der $L_{2,3}$-Kante $(l=1, p$-Orbitale) ermöglicht hingegen die Analyse von $s-(l=0)$ und d-Orbitalen $(l=2)$. Allerdings gelten die Auswahlregeln strikt nur für atomare Potenziale und können an den Absorptionskanten z. B. infolge einer Hybridisierung der Orbitale verletzt werden.

\subsection{MESSUNG VON NEXAFS-SPEKTREN}

NEXAFS-Spektren können auf verschiedene Arten aufgenommen werden: Entweder wird direkt die Intensität der Röntgenstrahlung gemessen, die durch die Probe hindurchdringt, oder der Absorptionskoeffizient wird indirekt bestimmt, indem z. B. Röntgenfluoreszenz oder AugerElektronen detektiert werden. Transmissionsmessungen werden im Bereich weicher Röntgenstrahlung insbesondere bei dünnen Proben bis zu einer Dicke von etwa $1 \mu \mathrm{m}$ verwendet. Für quantitative Analysen sollte die Probe darüber hinaus homogen und frei von Defekten sein, was allerdings besondere Anforderungen an die Probenpräparation stellt.

Für die Untersuchung dickerer Proben oder Proben geringer Konzentration $(<10 \%)$ sind indirekte Methoden besser geeignet. Aufgrund der Anregung eines kernnahen Elektrons bei der Absorption der Röntgenstrahlung entsteht in der entsprechenden Schale ein unbesetzter Elektronenzustand (",core hole"), der durch ein Elektron aus einer äußeren Schale besetzt werden kann. Beim Übergang zwischen den Schalen emittiert das Elektron wiederum ein Röntgenphoton. Die Intensität der Röntgenfluoreszenz ist ein Maß für die Absorptionswahrscheinlichkeit. Zudem können anhand der charakteristischen Energie der Fluoreszenzstrahlung die in der Probe vorhandenen Elemente identifiziert werden. Neben Fluoreszenz kann auch ein strahlungsloser Übergang in der Elektronenhülle des angeregten Atoms auftreten (Auger-Effekt). Dabei wird die frei werdende Energie infolge des Innerschalenübergangs nicht als Photon emittiert, sondern an ein weiteres, schwach gebundenes Elektron 
desselben Atoms übertragen. Dieses sogenannte Auger-Elektron verlässt das Atom und kann detektiert werden.

Im Bereich weicher Röntgenstrahlung dominiert der Auger-Prozess, sodass insbesondere für leichte Elemente Spektren durch Messung der Auger-Elektronenausbeute („Auger electron yield“) bestimmt werden. Die hochenergetischen Auger-Elektronen werden jedoch bereits nach einer kurzen Wegstrecke von wenigen Nanometern inelastisch gestreut und verlieren dadurch ihre Energie an andere Elektronen. Diese Sekundärelektronen können die Probe ebenso verlassen, sodass meist die Gesamtelektronenausbeute („total electron yield“) inklusive direkt angeregter Photoelektronen gemessen wird. Außerdem lassen sich mit dieser Methode aufgrund der geringen mittleren freien Weglänge der Elektronen nur Schichten bis zu einer Dicke von etwa $10 \mathrm{~nm}$ untersuchen. Die Detektion der Elektronen setzt des Weiteren ein Ultrahochvakuum mit Drücken von $10^{-10}$ bis $10^{-9}$ mbar voraus [217], um eine Verunreinigung der Probe mit Adsorbaten aus der Umgebungsluft zu vermeiden.

\subsection{STAND DER FORSCHUNG}

Strukturen an Absorptionskanten wurden für Röntgenstrahlen erstmals zweifelsfrei in Experimenten von Wilhelm Stenström, Hugo Fricke und Manne Siegbahn ${ }^{2}$ in den Jahren 1918 bis 1920 nachgewiesen [218]. Da geeignete Röntgenquellen und Detektoren insbesondere für detaillierte Untersuchungen der Feinstruktur fehlten, fand die Röntgenabsorptionsspektroskopie in den darauffolgenden Jahrzehnten kaum Beachtung. Erst zu Beginn der 1970er wurden Synchrotrone hoher Brillanz verfügbar, die zuverlässig Röntgenstrahlung bis zu einigen zehn Kiloelektronenvolt erzeugten. Dies ermöglichte die Analyse der Feinstruktur an Absorptionskanten zahlreicher Elemente, sodass die Methode nicht nur in der Physik, sondern auch in anderen Wissenschaftsdisziplinen (Chemie, Biophysik, Geologie ...) angewendet wird. Mittlerweile ist die Röntgenabsorptionsspektroskopie ein etabliertes Standardverfahren an Synchrotronen und über $50 \%$ der Anfragen nach Messzeit aus der Industrie gehen damit einher [219].

NEXAFS-Spektroskopie wird heutzutage meist in Kombination mit Röntgenmikroskopie eingesetzt (siehe Kap. 5 Spektromikroskopie), um die chemische Analyse mit einer räumlich hoch aufgelösten Abbildung der Probe zu verbinden. Die Proben können hierfür bei verschiedenen Bedingungen wie angelegten Magnetfeldern [220, 221] und hohen Temperaturen [222] mit einer spektralen Auflösung von bis zu E $/ \Delta E=10000$ [223] untersucht werden. In Pump-Probe-Experimenten lässt sich zudem mit einer Zeitauflösung bis in den Femtosekundenbereich etwa die

2 M. Siegbahn erhielt für seine röntgenspektroskopischen Entdeckungen und Forschungen 1924 den Nobelpreis für Physik. 
Elektronendynamik z. B. photoinduzierter Phasenübergänge [224] charakterisieren. Darüber hinaus müssen die Messungen nicht unter Vakuumbedingungen stattfinden: Ausgasende Proben werden stattdessen in heliumgefüllten Kammern bei Atmosphärendruck analysiert [225], und Fluidzellen ermöglichen zeitaufgelöste In-situ-Messungen [226, 227].

Wie die Röntgenmikroskopie wird die Absorptionsspektroskopie fast ausschließlich an Synchrotronen angewendet. Infolge der kontinuierlichen Weiterentwicklung kompakter Röntgenquellen sind NEXAFS-Untersuchungen jedoch seit einigen Jahren auch im Labor möglich. Da$\mathrm{zu}$ werden überwiegend laserinduzierte Plasmaquellen eingesetzt, die je nach Targetmaterial breitbandig weiche Röntgenstrahlung zwischen 50 und $1250 \mathrm{eV}$ erzeugen.

Auf der Basis eines laserinduzierten Plasmas mit Festkörpertarget entwickelten Vogt et al. und Kanngießer et al. Laborspektrometer, in denen als dispersives Element reflektierende „off-axis"-Zonenplatten eingesetzt werden $[228,229]$. Damit wird eine hohe spektrale Auflösung von bis $z u E / \Delta E \approx 950$ an der Kohlenstoff-K-Kante $(E=284 \mathrm{eV})$ erreicht. Die Verwendung von "off-axis"-Zonenplatten hat allerdings zwei entscheidende Nachteile: Erstens sind für die hohe Auflösung sehr große Abstände von etwa $4 \mathrm{~m}$ zwischen Quelle und Detektor notwendig. Zweitens kann mit einer Zonenplatte nur ein begrenzter Spektralbereich bei dieser hohen Auflösung abgebildet werden. Für verschiedene Absorptionskanten müssen daher unterschiedliche Zonenplatten verwendet werden, von denen jede speziell entsprechend der Photonenenergie parametrisiert werden muss.

Als Alternative zu den „off-axis“-Zonenplatten können Transmissionsoder Reflexionsgitter als dispersives Element genutzt werden. In der Abteilung Optik / Kurze Wellenlängen des Laser-Laboratoriums Göttingen e.V. (LLG) wurde in den letzten 10 Jahren eine kompaktes Röntgenspektrometer mit Reflexionsgitter entwickelt [27] und stetig optimiert. Zahlreiche Messungen an Proben nicht nur in Transmissions-, sondern auch in Reflexionsgeometrie belegen für den Spektralbereich von 250 bis 530 eV eine sehr gute Übereinstimmung mit Synchrotrondaten [2729, 230]. Darüber hinaus wurden bereits Pump-Probe-Experimente mit Subnanosekundenzeitauflösung durchgeführt [231].

Im Rahmen dieser Arbeit wurde das Spektrometer erneut weiterentwickelt, sodass z. B. Untersuchungen oberhalb der Sauerstoff-K-Kante $(530 \mathrm{eV})$ insbesondere von 3d-Übergangsmetallen durchgeführt werden konnten. Zudem wurde eine heliumgefüllte Probenkammer getestet, die Messungen von Proben unter Atmosphärendruck ermöglicht [232]. Der gegenwärtige Aufbau des Spektrometers und die damit erzielten Messergebnisse werden in den Abschnitten 4.4 bzw. 4.5 beschrieben. 
Ein zum LLG-Aufbau quasi identisches NEXAFS-Spektrometer für den Energiebereich von 250 bis $500 \mathrm{eV}$ wurde Anfang 2018 auch in der AG Fiedorowicz realisiert [233].

Alternativ zu laserinduzierten Plasmaquellen werden seit kurzem hohe Harmonische für die NEXAFS-Spektroskopie genutzt: Biegert et al. erzeugten dazu ein kontinuierliches Spektrum im Energiebereich von 225 bis $530 \mathrm{eV}$ und untersuchten die Kohlenstoff-K- und Titan- $\mathrm{L}_{2,3}$-Kante $[71,234]$. Aufgrund der kurzen Pulslänge eignen sich hohe Harmonische insbesondere für Pump-Probe-Experimente, um z. B. die Elektronendynamik von Atomen und Molekülen an Oberflächen mit Attosekundenzeitauflösung zu untersuchen [235]. Allerdings ist die erreichbare Photonenenergie derzeit auf etwa $530 \mathrm{eV}$ begrenzt.

\subsection{LABORRÖNTGENSPEKTROMETER}

NEXAFS-Messungen im Zuge dieser Arbeit wurden mit einem kompakten Röntgenspektrometer durchgeführt, dessen Aufbau in Abbildung 4.3 gezeigt ist. Als Quelle breitbandiger Strahlung wird dabei ein von einem Pikosekundenlaser (Ekspla, Wellenlänge 1064 nm, Pulslänge 170 ps, Pulsenergie bis $500 \mathrm{~mJ}$ ) induziertes Kryptonplasma in Kombination mit
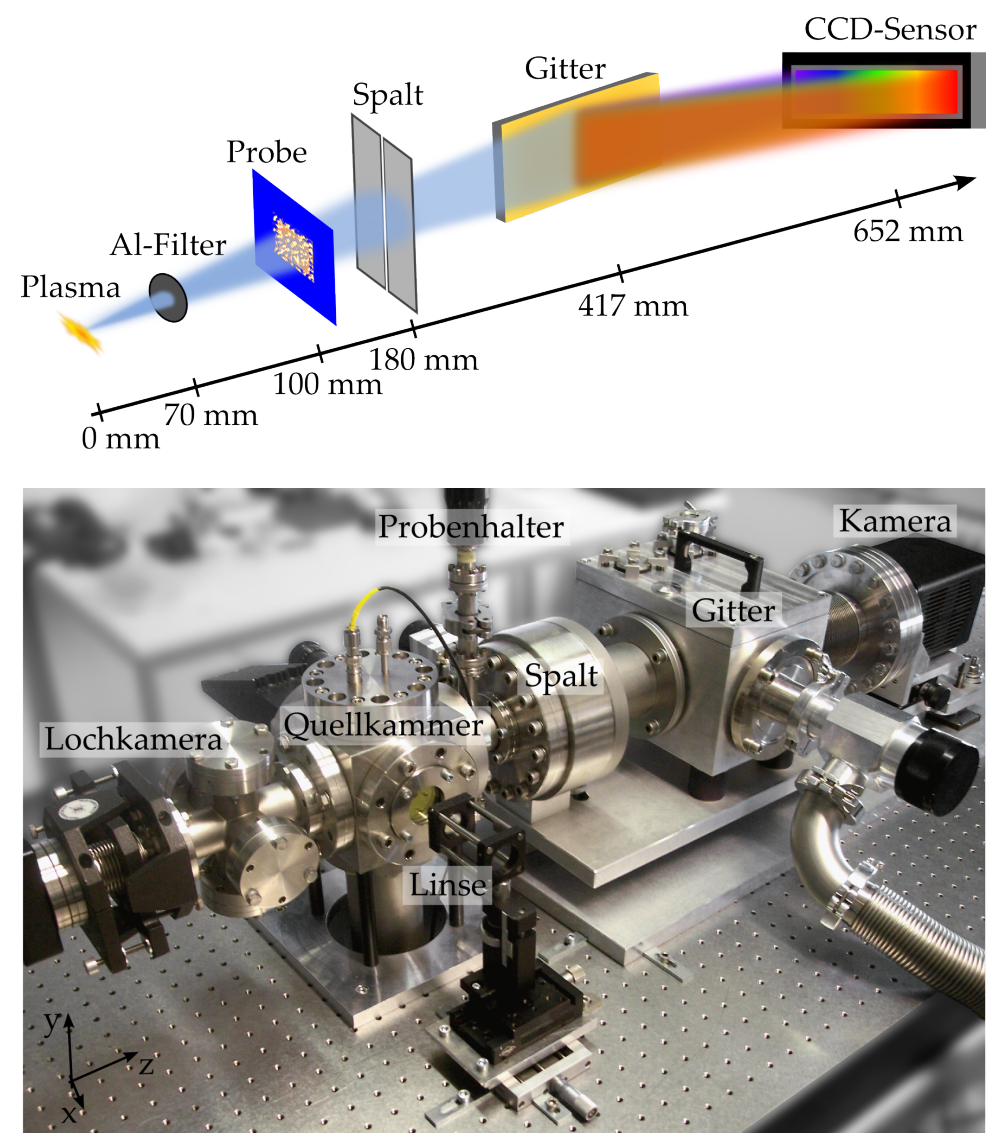

Abbildung 4.3: Schema (oben) und Foto (unten) des Röntgenspektrometers 
einem Aluminiumfilter (Dicke $150 \mathrm{~nm}$ ) genutzt (siehe Abs. 2.3.3 und 2.3.4). Das Spektrometer selbst besteht aus einem Eintrittspalt (Breite 10 bis $300 \mu \mathrm{m}$ ), einem Reflexionsgitter (Hitachi, $30 \mathrm{~mm} \times 50 \mathrm{~mm}$, Energiebereich 250 bis $1250 \mathrm{eV}$, mittlere Gitterkonstante 2400 Linien/mm) und einer Kamera (Roper Scientific, $2048 \times 512$ Pixel, Pixelgröße 13,5 $\mu \mathrm{m}$ ). Die weiche Röntgenstrahlung trifft unter streifendem Einfall mit einem Winkel von $1,3^{\circ}$ auf die goldbeschichtete Gitteroberfläche (siehe Abb. 4.4). Um die Effizienz des Gitters für die 1. Beugungsordnung zu maximie-

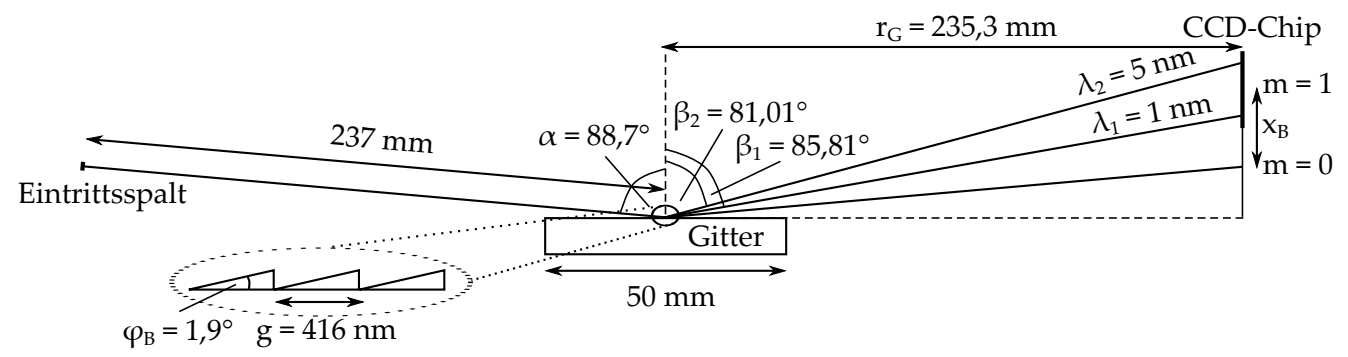

Abbildung 4.4: Skizze mit Spezifikationen des Reflexionsgitters

ren33, weisen die Gitterlinien einen dreieckigen Querschnitt auf. Die so entstehenden Stufen sind um den Blazewinkel gegen die Gitteroberfläche geneigt. Das im Spektrometer eingesetzte Gitter ist für eine Photonenenergie von $830 \mathrm{eV}$ optimiert. Der entsprechende Blazewinkel beträgt $\varphi_{\mathrm{B}}=1,9^{\circ}$. Da das Gitter konkav geformt ist (Krümmungsradius $r_{\mathrm{K}}=15920 \mathrm{~mm}$ ) und darüber hinaus eine variierende Liniendichte (2000 bis 2936 Linien/mm) aufweist, wird der Eintrittsspalt $(237 \mathrm{~mm}$ vor dem Gitter) auf eine Ebene senkrecht zur Ausbreitungsrichtung der Strahlung abgebildet („flat focal field“) [236]. An diese Position - 235,3 mm vom Gitter entfernt - wird der „back-side-illuminated“ CCD-Chip gestellt. Aufgrund der rückwärtigen Belichtung des CCD-Chips wird für weiche Röntgenstrahlung eine Quanteneffizienz von 50 bis $85 \%$ erreicht. Um thermisches Rauschen während der Aufnahme von Spektren zu minimieren, wird der CCD-Chip auf eine Temperatur von $-30^{\circ} \mathrm{C}$ gekühlt.

Die Proben sind auf einer Lineardurchführung befestigt, um sie optimal im Strahlengang zu positionieren. Der Abstand von der Plasmaquelle zur Probe beträgt etwa $100 \mathrm{~mm}$, der von Probe zum Eintrittsspalt etwa $80 \mathrm{~mm}$. Damit konnten die Abstände gegenüber früheren Aufbauten deutlich um ca. $120 \mathrm{~mm}$ zwischen Quelle und Probe und sogar $345 \mathrm{~mm}$ zwischen Probe und Spalt verringert werden. Infolgedessen steigt das Signal-Rausch-Verhältnis, insbesondere im Energiebereich $>500 \mathrm{eV}$ ist die Intensität der Spektren bei diesem Aufbau hinreichend hoch, um verlässlich NEXAFS-Messungen durchzuführen.

Aufgrund der geringen mittleren freien Weglänge weicher Röntgenstrahlung in Luft (wenige Millimeter) ist bei NEXAFS-Messungen ein

3 Der Hersteller gibt über den gesamten Energiebereich eine theoretische Effizienz von etwa 30 bis $40 \%$ für die 1 . Beugungsordnung an. 
Hochvakuum mit Drücken kleiner $10^{-2}$ mbar erforderlich. Somit können keine ausgasenden Proben (z. B. Flüssigkeiten oder adsorbierte Gase) untersucht werden. Darüber hinaus sind Proben wie Proteine, DNS und hydratisierte Verbindungen von Übergangsmetallen nicht messbar, da sich deren Struktur im Hochvakuum infolge einer Dehydratisierung bzw. der Wechselwirkung zwischen Substrat und Adsorbat ändert. Wie in Abschnitt 4.3 bereits erwähnt, werden an Synchrotronen daher Proben in heliumgefüllten Kammern analysiert [225]. Nach diesem Vorbild wurde eine kompakte Probenhalter für das Spektrometer entwickelt, mit der die Proben auch unter Atmosphärendruck entweder in Luft oder einer Heliumumgebung analysiert werden können [230, 232]. Die Probe wird dabei von zwei $150 \mathrm{~nm}$ dicken $\mathrm{Si}_{3} \mathrm{~N}_{4}$-Membranen (Fenstergröße $1 \mathrm{~mm} \times 1 \mathrm{~mm}$ ) von der Vakuumkammer separiert (siehe Abb. 4.5). Der Abstand der beiden Membranen beträgt $4 \mathrm{~mm}$, sodass eine ausreichend hohe Transmission der weichen Röntgenstrahlung gewährleistet ist.
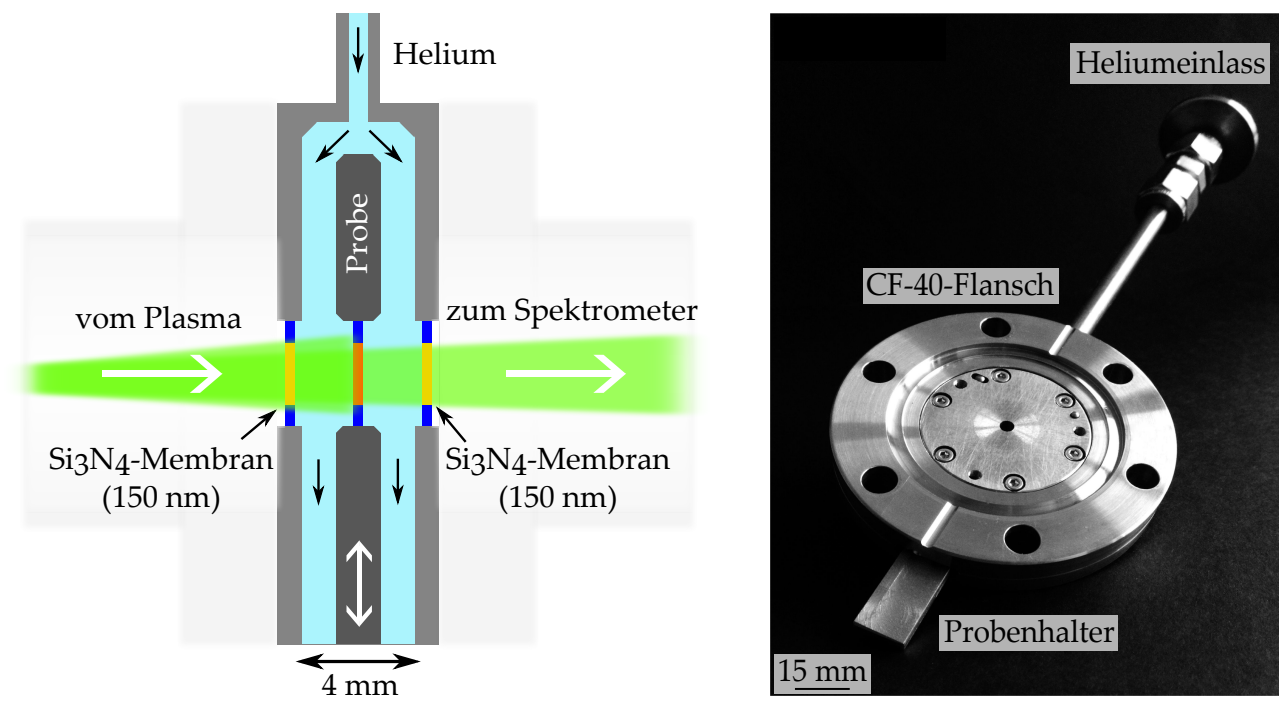

Abbildung 4.5: Schematische Darstellung (links) und Foto (rechts) der Probenkammer für Atmosphärendruck.

Bevor die Ergebnisse der NEXAFS-Experimente an ausgewählten Materialsystemen vorgestellt werden (siehe Abs. 4.5), werden in den nächsten Abschnitten zunächst Aspekte von Gitterjustage und -kalibration sowie der Bestimmung des Auflösungsvermögens diskutiert.

\subsubsection{GITTERJUSTAGE UND -KALIBRATION}

Infolge der abbildenden Eigenschaften des Reflexionsgitters hängt die Beugungseffizienz und das Auflösungsvermögen wesentlich von einer exakten Ausrichtung des Gitters im Spektrometer ab. Zur Justage wird daher in einem ersten Schritt ein HeNe-Laser durch den Aufbau geführt, sodass dieser von der Düsenmitte aus den Eintrittsspalt des Spektrome- 
ters mittig trifft. Im Abstand von etwa $2 \mathrm{~m}$ wird die Position des Laserspots auf einem Schirm markiert. Das Gitter wird daraufhin parallel zur optischen Achse in den Strahlengang gebracht, bis die Hälfte des Laserspots verdeckt ist, ohne dass ein Beugungsreflex erkennbar wäre.

Die erste Beugungsordnung liegt unter einem Winkel von 1,30 zur Gitterebene. Das Gitter wird daher soweit verkippt, bis der Beugungsreflex genau unter 2,6 $6^{\circ}$ zur ursprünglichen Markierung auf dem Schirm zu sehen ist. Gleichzeitig wird sichergestellt, dass das Beugungsmuster parallel zur x-Achse verläuft (Koordinatensystem siehe Abb. 4.3).

Die Feinjustage des Gitters erfolgt anschließend mit einem Stickstoffplasma, dessen Emissionsspektrum auf der Kamera mit rückseitig gedünntem CCD-Chip aufgenommen wird. Das Gitter wird dazu so lange verkippt, bis die jeweilige Position der Stickstofflinien über den gesamten CCD-Chip auf dieselben Pixelspalten fällt. Zudem wird darauf geachtet, dass alle Pixelreihen gleichmäßig ausgeleuchtet sind. Die Justage führt nicht nur zu einer verbesserten Auflösung, sondern auch zu einer knapp $50 \%$ höheren Intensität des Spektrums.

Um das Spektrometer zu kalibrieren, eignen sich Emissionsspektren leichter Elemente wie Stickstoff und Sauerstoff (siehe Abb. 2.10). Die einzelnen Linien werden den entsprechenden Übergängen zugeordnet und mit tabellierten Daten aus der "NIST Atomic Spectra Database“ [237] verglichen. Allerdings wird auf diese Weise nur ein Energiebereich von 400 bis 700 eV abgedeckt. Daher werden zur Kalibration zusätzlich Proben (Polyimid, Siliziumnitrid, Goethit und elementares Kupfer) vermessen, deren Absorptionsspektren sehr genau an Synchrotronstrahlquellen bestimmt wurden. Den Pixeln charakteristischer Peaks zwischen 285 und $930 \mathrm{eV}$ an der Kohlenstoff-, Stickstoff- und Sauerstoff-K-Kante bzw. an der Eisen- und Kupfer-L-Kante wurden anschließend die entsprechenden Photonenenergien zugeordnet (Daten siehe Tab. A.6). Abschließend wurde an die Datenpunkte ein Polynom 5. Grades angepasst (Fitparameter siehe Tab. A.7), das eine Umrechnung der Pixel in Photonenenergie ermöglicht.

\subsubsection{AUFLÖSUNGSVERMÖGEN}

Eine wichtige Kenngröße von Spektrometern ist deren Auflösungsvermögen A. Es beschreibt die Fähigkeit des Spektrometers, zwei nah beieinanderliegende Spektrallinien voneinander zu trennen und wird üblicherweise als dimensionslose Größe

$$
A=\frac{E}{\Delta E}
$$

angegeben. Dabei ist $E$ die mittlere Energie und $\Delta E$ die Energiedifferenz zwischen den beiden Linien. Gemäß dem Rayleigh-Kriterium gelten die 
Spektrallinien als unterscheidbar, wenn das Maximum der einen Linie in das Minimum der anderen fällt.

Das Auflösungsvermögen des Spektrometers wird von Parametern der Plasmaquelle, der Breite des Eintrittsspalts, der Anzahl ausgeleuchteter Gitterlinien, der Ausrichtung des Gitters und der Pixelgröße des CCD-Chips bestimmt. In der Bildebene lässt sich das Auflösungsvermögen unter Vernachlässigung der abbildenden Eigenschaften des Gitters ausgehend von der Gittergleichung berechnen

$$
\mathrm{m} \lambda=\mathrm{g}[\sin (\alpha)+\sin (\beta)] \quad \Leftrightarrow \quad \mathrm{E}(\beta)=\frac{\mathrm{mhc}_{0}}{\mathrm{~g}[\sin (\alpha)+\sin (\beta)]},
$$

wobei $m=1,2,3 \ldots$ die Beugungsordnung, $\lambda$ die Wellenlänge, $E$ die Photonenenergie, $h=2 \pi \hbar$ das Plancksche Wirkungsquantum, $c_{0}$ die Lichtgeschwindigkeit, $g$ die mittlere Gitterkonstante, $\alpha$ den Einfallswinkel und $\beta$ den Beugungswinkel bezeichnen. Des Weiteren gilt für den Beugungswinkel unter Berücksichtigung des Abstands $r_{G}$ zwischen Gittermitte und Detektor sowie dem Abstand $x_{\mathrm{B}}$ zwischen der o. Ordnung und dem gebeugten Strahl auf dem CCD-Chip (siehe Abb. 4.4)

$$
\sin (\beta)=\sqrt{\frac{1}{1+\left(\frac{x_{B}}{r_{G}}\right)^{2}}} .
$$

Aus den Gleichungen (4.3) und (4.4) folgt für den Abstand $x_{B}$ mit $r_{G}>0$

$$
x_{B}=r_{G} \sqrt{\frac{1}{\left[\frac{m h c_{0}}{g E}-\sin (\alpha)\right]^{2}-1}}
$$

und die Änderung der Energie in Abhängigkeit des Abstands $x_{B}$ auf dem Detektor ergibt sich zu:

$$
\frac{\partial E}{\partial x_{B}}=\frac{g E^{2}}{m h c_{0}} \frac{r_{G} x_{B}}{\left(r_{G}^{2}+x_{B}^{2}\right)^{3 / 2}} .
$$

Aufgrund der endlichen Pixelbreite $\Delta x$ des CCD-Chips gilt für die kleinste messbare Energiedifferenz

$$
\Delta \mathrm{E}=\frac{\partial \mathrm{E}}{\partial \mathrm{x}_{\mathrm{B}}} \Delta \mathrm{x}
$$

und demnach für das Auflösungsvermögen

$$
\frac{E}{\Delta E}=\frac{E}{\frac{\partial E}{\partial x_{B}} \Delta x} .
$$



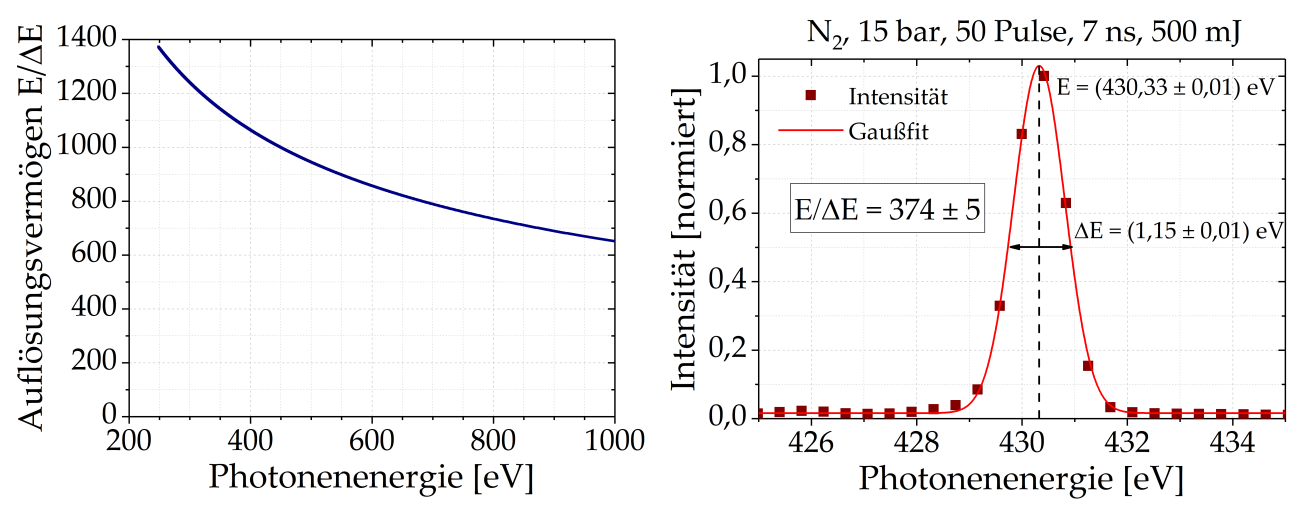

Abbildung 4.6: Anhand der Gitterparameter und Pixelbreite des CCD-Chips berechnetes (links) und aus Halbwertsbreite der Spektrallinie von Stickstoff bei $E=430 \mathrm{eV}$ experimentell bestimmtes (rechts) Auflösungsvermögen des Röntgenspektrometers. Eintrittsspalt (Breite $100 \mu \mathrm{m}$ ) und Plasmaquelle sind für das theoretische Auflösungsvermögen an dieser Stelle nicht berücksichtigt.

Abbildung 4.6 zeigt das nach Gleichung (4.8) berechnete Auflösungsvermögen als Funktion der Photonenenergie E für die erste Beugungsordnung $(m=1)$ und die Parameter Pixelbreite $\Delta x=13,5 \mu \mathrm{m}$, mittlere Gitterkonstante $\mathrm{g}=2400$ Linien/mm, Abstand Gitterkammer - Detektor $r_{\mathrm{G}}=235,3 \mathrm{~mm}$ sowie Einfallswinkel $\alpha=88,7^{\circ}$. Das theoretische Auflösungsvermögen geht mit steigender Photonenenergie von zunächst etwa $\mathrm{E} / \Delta \mathrm{E}=1400$ bei $250 \mathrm{eV}$ auf ungefähr $\mathrm{E} / \Delta \mathrm{E}=650$ bei $1000 \mathrm{eV}$ zurück. Vorausgesetzt wird dabei eine exakte Ausrichtung des Gitters (vgl. Abs. 4.4.1). Bereits ab einer Winkeldifferenz von $\Delta \alpha=0,2^{\circ}$ zum optimalen Einfallswinkel wird die Röntgenstrahlung statt in ein "flat focal field“ in eine gekrümmte Ebene und damit verzerrt auf den ebenen CCD-Chip abgebildet [238] (siehe Abb. 4.7). Das Auflösungsvermögen kann demnach als Kriterium für die Gitterjustage herangezogen werden.

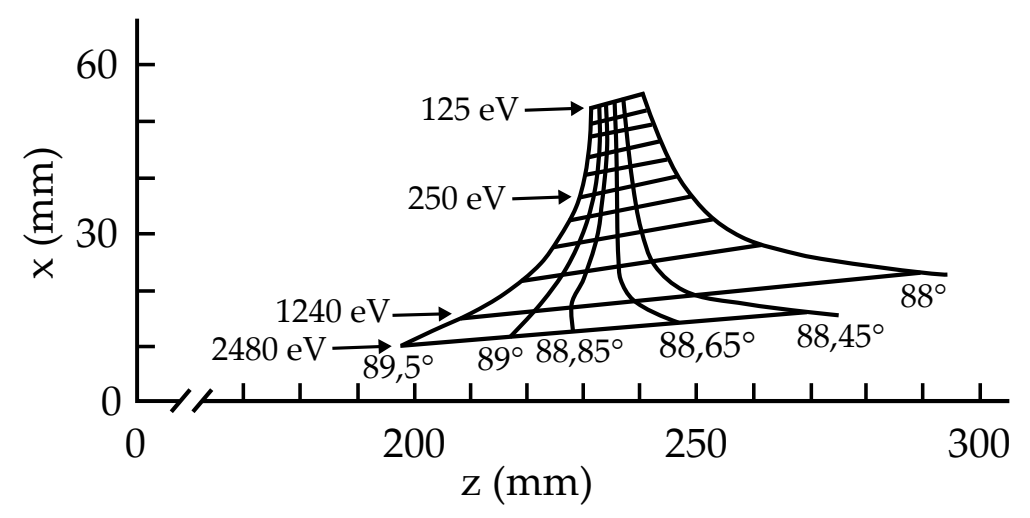

Abbildung 4.7: Berechnete Brennebenen des Reflexionsgitters in Abhängigkeit des Einfallswinkels (übernommen aus [238]) 
Experimentell wird das Auflösungsvermögen meist nicht nach dem Rayleigh-Kriterium, sondern anhand der Breite einer einzelnen Spektrallinie bestimmt, deren natürliche Linienbreite unterhalb des Auflösungsvermögens des Spektrometers liegt. Abbildung 4.6 rechts zeigt die Spektrallinie von Stickstoff bei $E=430 \mathrm{eV}$ und den dazugehörigen Gaußfit. Aus der Halbwertsbreite der Gaußkurve wird das Auflösungsvermögen des Spektrometers zu $(\mathrm{E} / \Delta \mathrm{E})_{\exp }=374 \pm 5$ berechnet und liegt damit etwa um den Faktor 2,75 unter dem theoretischen Wert von $E / \Delta E \approx 1030$. Diese Abweichung hat mehrere Ursachen: Bisher wurde zur Bestimmung des theoretischen Auflösungsvermögens ein idealer CCD-Chip angenommen, bei dem ein Röntgenphoton, das ein Pixel belichtet, auch in diesem Pixel detektiert wird. Allerdings breiten sich die Elektronen, die infolge der Absorption eines hochenergetischen Photons erzeugt werden, innerhalb der Siliziumschicht des CCD-Chips aus. Dinardo et al. [239] konnten zeigen, dass sich 80 \% der Elektronen auf eine Kreisfläche mit einem Durchmesser von etwa $25 \mu \mathrm{m}$ verteilen. Das theoretische Auflösungsvermögen des Spektrometers ist dementsprechend aufgrund der effektiv größeren Pixel etwa um den Faktor 2 auf $E / \Delta E \approx 550$ verringert. Das Auflösungsvermögen wird zusätzlich durch die Breite des Eintrittsspalts und die Plasmaquelle selbst beeinflusst. Beide Aspekte werden im Folgenden diskutiert.

\section{EINFLUSS DER SPALTGRÖSSE}

Um den Einfluss des Eintrittsspalts auf das Auflösungsvermögen des Spektrometers zu ermitteln, wurden Emissionsspektren von Stickstoff für verschiedene Spaltbreiten $b$ aufgenommen, und an die Spektrallinie bei $E=430 \mathrm{eV}$ jeweils eine Gaußkurve angepasst. Die Fläche der Gaußkurve ist ein Maß für die Intensität der Stickstofflinie; aus der Halbwertsbreite wurde - wie bereits beschrieben - das Auflösungsvermögen bestimmt. In Abbildung 4.8 sind beide Größen als Funktion der Spaltbreite aufgetragen. Je schmaler der Spalt ist, desto geringer ist die Intensität, und desto besser ist die Stickstofflinie aufgelöst. Im Gegensatz zur Intensität ist der Anstieg des Auflösungsvermögens allerdings nicht linear zur Spaltbreite, sondern flacht für $b<100 \mu \mathrm{m}$ zunehmend $a b$. Für Messungen wurde daher der Eintrittsspalt auf $100 \mu \mathrm{m}$ eingestellt, um eine hinreichend hohe Intensität bei lediglich geringfügig (10\%) verringerter Auflösung zu erreichen.

EINFLUSS DER PLASMAQUELLE

Obwohl das Auflösungsvermögen für schmalere Spalte ansteigt, weicht es selbst für die kleinste Spaltbreite von $\approx 10 \mu \mathrm{m}$ um einen Faktor 1,33 vom theoretischen Wert ab. Diese Diskrepanz ist - zumindest teilweise - 


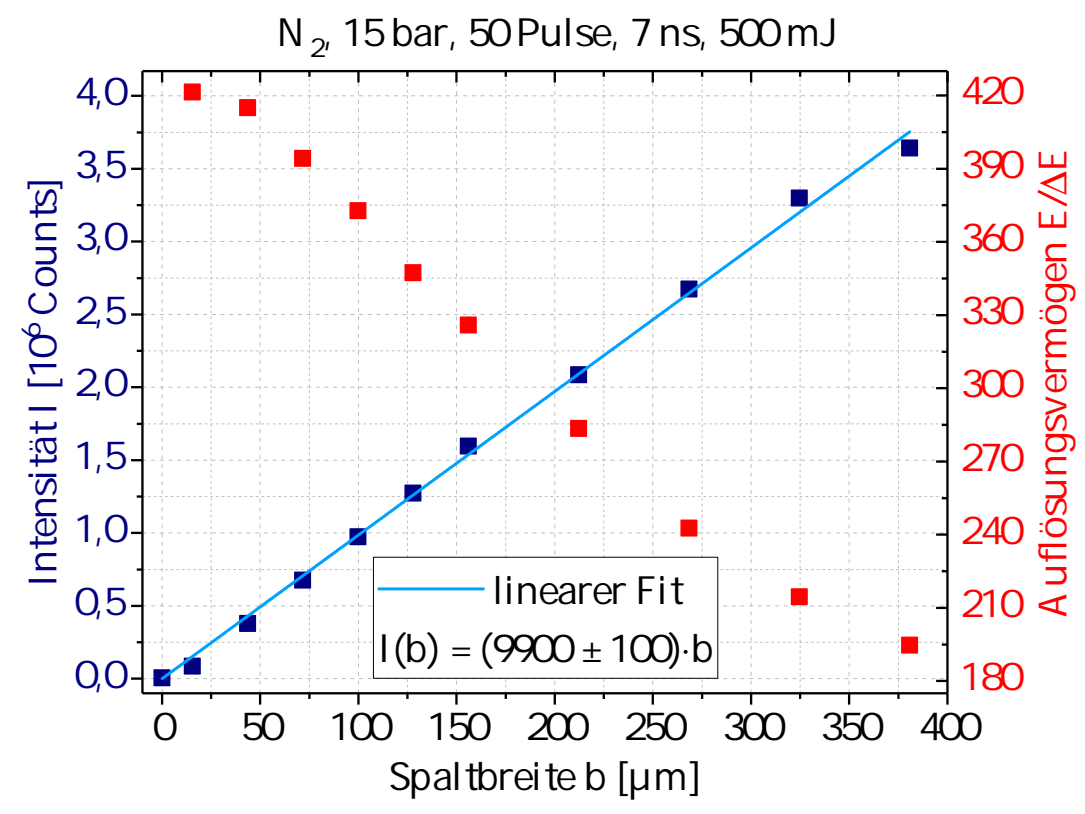

Abbildung 4.8: Intensität der Stickstofflinie bei $E=430 \mathrm{eV}$ und Auflösungsvermögen des Spektrometers in Abhängigkeit von der Breite des Eintrittsspalts

durch die Ausdehnung $\left(1 / \mathrm{e}^{2}: 0,94 \mathrm{~mm} \times 0,35 \mathrm{~mm}\right)$ und Lageinstabilität des Plasmas (etwa $10 \mu \mathrm{m}$ entlang der $x$-Achse) bedingt (siehe Tab. 2.3).

Inwiefern sich die räumlichen Schwankungen des Plasmas auf das Auflösungsvermögen auswirken, wurde überprüft, indem die Spektrallinie des Stickstoffs bei $E=430 \mathrm{eV}$ im Einzelpuls und über 5, 10 und 50 Pulse integriert für zwei verschiedene Spaltbreiten von $100 \mu \mathrm{m}$ bzw. $15 \mu \mathrm{m}$ aufgenommen wurden (siehe Abbildung 4.9). Da unabhängig von der Pulsanzahl keine Unterschiede in der Stickstofflinie zu erkennen sind, lässt sich die Instabilität des Plasmas entlang der $x$-Achse als Ursache für das verringerte Auflösungsvermögen ausschließen.

Im Gegensatz zu Spektrometern für sichtbares Licht wird der Eintrittsspalt in diesem Aufbau nicht mit einem parallelen, sondern mit einem divergenten Strahl beleuchtet. Die weiche Röntgenstrahlung trifft folglich je nach Ursprung im Plasma mit geringfügig unterschiedlichen Einfallswinkeln auf das Gitter und wird an verschiedene Positionen auf den CCD-Chip abgebildet. Je größer das Plasma ist, desto stärker wird demnach das Auflösungsvermögen verringert. Um die Quellgröße und damit die Divergenz zu verkleinern, wurde zusätzlich zum $100 \mu \mathrm{m}$ breiten Eintrittsspalt des Spektrometers ein ebenso breiter Spalt in den Strahlengang eingebracht, und zwar im Abstand von etwa $15 \mathrm{~mm}$ zum Plasma. Die Stickstofflinie und das entsprechende Auflösungsvermögen mit und ohne Quellspalt sind in Abbildung 4.10 gezeigt. Mit Quellspalt beträgt das Auflösungsvermögen $(E / \Delta E)_{\exp } \approx 500$ und ist damit im Vergleich zur Messung ohne zusätzlichen Spalt um etwa ein Drittel größer. Würden statt der $100 \mu \mathrm{m}$ breiten Spalte jeweils kleinere Aperturen verwen- 

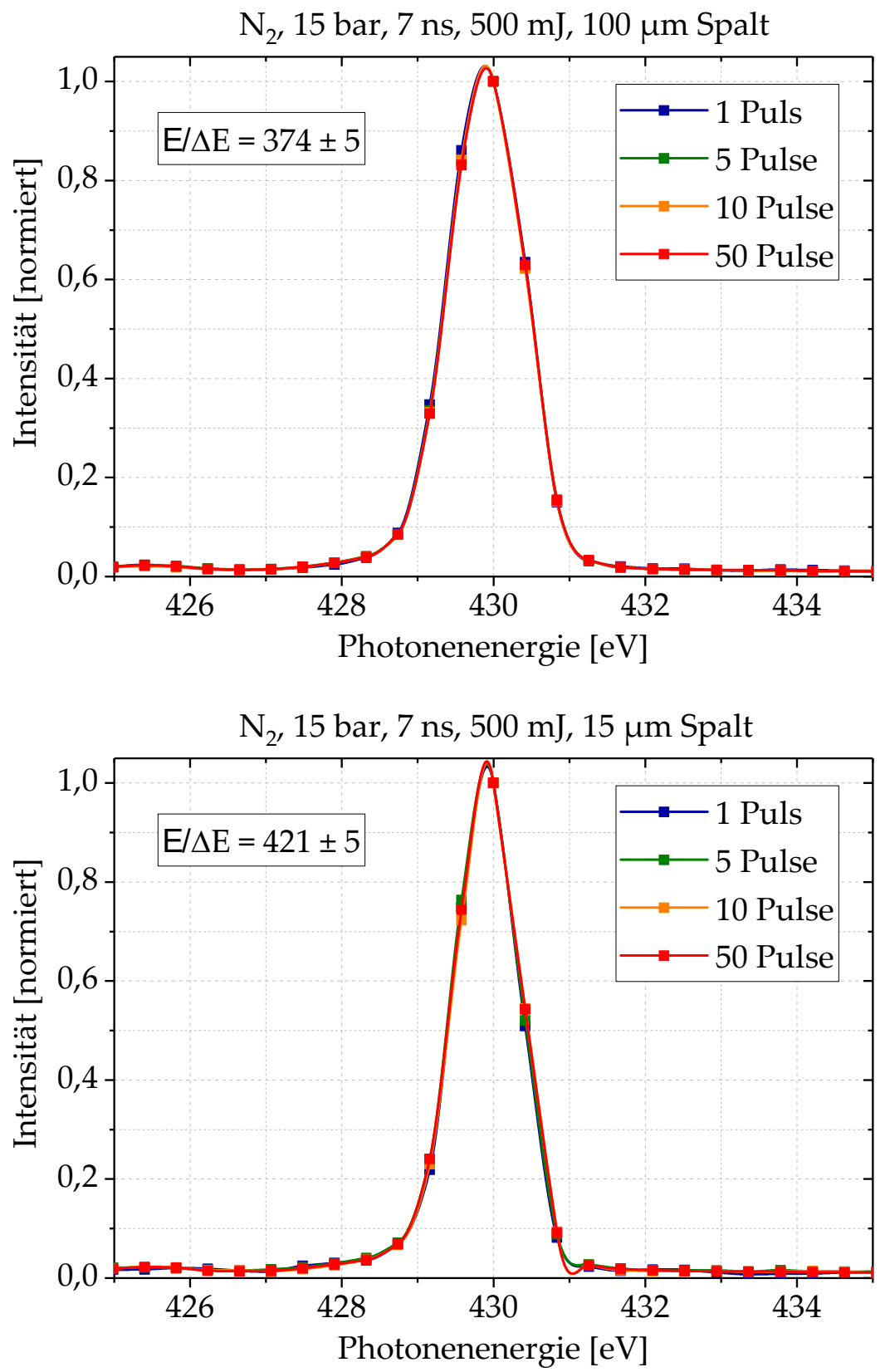

Abbildung 4.9: Spektrallinie des Stickstoffs bei $E=430 \mathrm{eV}$ integriert über unterschiedliche Anzahlen von Pulsen für $100 \mu \mathrm{m}$ (oben) bzw. $15 \mu \mathrm{m}$ (unten) breiten Eintrittsspalt 


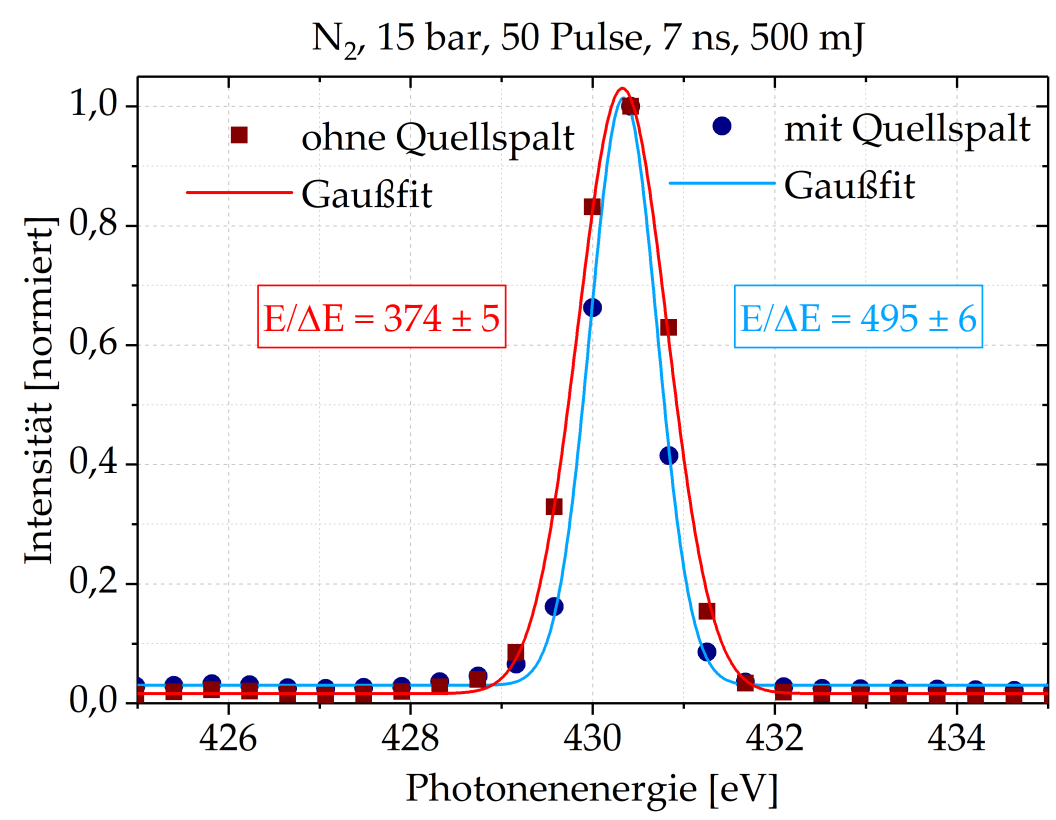

Abbildung 4.10: Spektrallinie des Stickstoffs bei $E=430 \mathrm{eV}$ und daraus ermittelter Wert des Auflösungsvermögens mit und ohne Quellspalt (Breite $100 \mu \mathrm{m}$ )

det, könnte das Auflösungsvermögen des Röntgenspektrometers nahezu auf den theoretischen Wert von $E / \Delta E=550$ erhöht werden. Allerdings würde die Intensität bei sehr schmalen Spalten zu stark abnehmen (siehe Abb. 4.8). Ziel muss es deshalb sein, dass Plasma an sich zu verkleinern, ohne die Intensität zu verringern. Dazu eignet sich beispielsweise die in Abschnitt 2.3.4 beschriebene Fokussierung unter kleineren Winkeln $\left(30^{\circ}\right.$ statt $90^{\circ}$ ), die zukünftig berücksichtigt werden sollte.

\subsection{UNTERSUCHTE MATERIALSYSTEME}

Im folgenden Abschnitt werden die Ergebnisse der NEXAFS-Messungen verschiedener Materialien diskutiert. Die Proben wurden dabei so ausgewählt, dass die Absorptionskanten der entsprechenden Elemente über einen großen Energiebereich verteilt sind. Insbesondere soll gezeigt werden, dass mit dem verbesserten Aufbau des Laborröntgenspektrometers und der neuen heliumgefüllten Probenkammer Untersuchungen im Energiebereich oberhalb von $500 \mathrm{eV}$ verlässlich möglich sind. Die optische Dichte der Proben wird gemäß Lambert-Beer'schem Gesetz (siehe Gl. (4.I)) berechnet.

Wenn nicht anders beschrieben, werden die Proben in wässriger Lösung auf eine $100 \mathrm{~nm}$ dicke $\mathrm{Si}_{3} \mathrm{~N}_{4}$-Membran aufgetragen. Anschließend wird das Wasser ggf. unter Verwendung eines Heißluftföhns verdampft, um eine möglichst gleichmäßige Verteilung des Probenmaterials auf der Membran zu erreichen (siehe Abb. 4.II). 

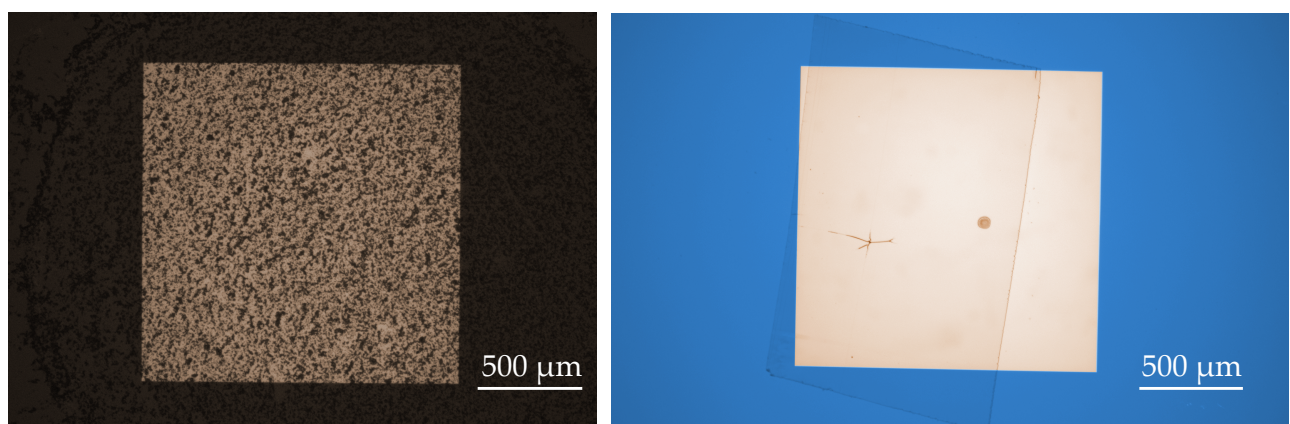

Abbildung 4.11: Mikroskopaufnahme (5fache Vergrößerung, Hellfeld) des Schichtsilikats CCa-2 Chlorit (links) und einer $200 \mathrm{~nm}$ dicken Agar-100-Probe (Epoxidharz, rechts) auf $\mathrm{Si}_{3} \mathrm{~N}_{4}$-Membran $(100 \mathrm{~nm}$ )

\subsubsection{EPOXIDHARZ AGAR-100}

Neben der Homogenität beeinflusst auch die Schichtdicke der Probe die Qualität und damit die Aussagekraft von NEXAFS-Spektren. Anhand sechs unterschiedlich dicker Agar-100-Proben wurde untersucht, inwiefern sich die Spektren in Abhängigkeit der Probendicke verändern. Agar-100 (CAS-Nummer 90 529-77-4) ist ein Epoxidharz, das häufig als Einbettmedium für Proben in der Transmissionselektronenmikroskopie eingesetzt wird ${ }^{4}$. Es besitzt $\mathrm{u}$. a. Kohlenstoffverbindungen $(\mathrm{C}=\mathrm{C}-$ Doppelbindung) mit Methylgruppe ( $\mathrm{CH}_{3}$-Bindung, siehe Abb. 4.12). Die Proben wurden von Dietmar Riedel (Max-Planck-Institut für biophysikalische Chemie) mit einem Ultramikrotom auf Dicken von 100 bis $1400 \mathrm{~nm}$ geschnitten und jeweils auf einer $\mathrm{Si}_{3} \mathrm{~N}_{4}$-Membran abgelegt (Dicke 100 nm, siehe Abb. 4.11).

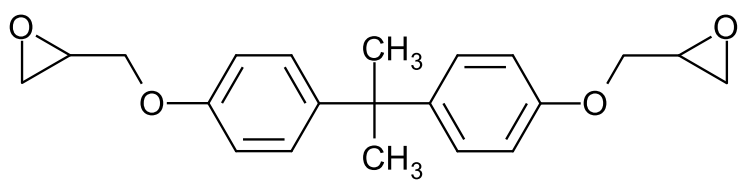

Abbildung 4.12: Strukturformel eines typischen Epoxidharzes

In Abbildung 4.13 sind die NEXAFS-Spektren dieser Agar-100-Proben an der Kohlenstoff-K-Kante dargestellt. Der charakteristische Peak der $\mathrm{C}=\mathrm{C}$-Doppelbindung bei $284,7 \mathrm{eV}$ ist in allen Spektren eindeutig sichtbar, ist aber umso stärker ausgeprägt, je dicker die Probe ist. Ab einer Probendicke von etwa $600 \mathrm{~nm}$ ist die optische Dichte durch Modulationen aus dem Emissionsspektrum des Kryptons geprägt. Der ohnehin etwas verbreiterte Peak der $\mathrm{CH}_{3}$-Bindung $(288,7 \mathrm{eV})$ lässt sich daher mit zunehmender Probendicke immer schlechter vom Rauschen unterschei-

4 Nicht zu verwechseln mit dem Galactose-Polymer Agar, dass u. a. in der Biologie als Nährboden für Mikroorganismen genutzt wird. 
Kr, 20 bar, 10000 Pulse, 7 ns, 500 mJ, Al-Filter
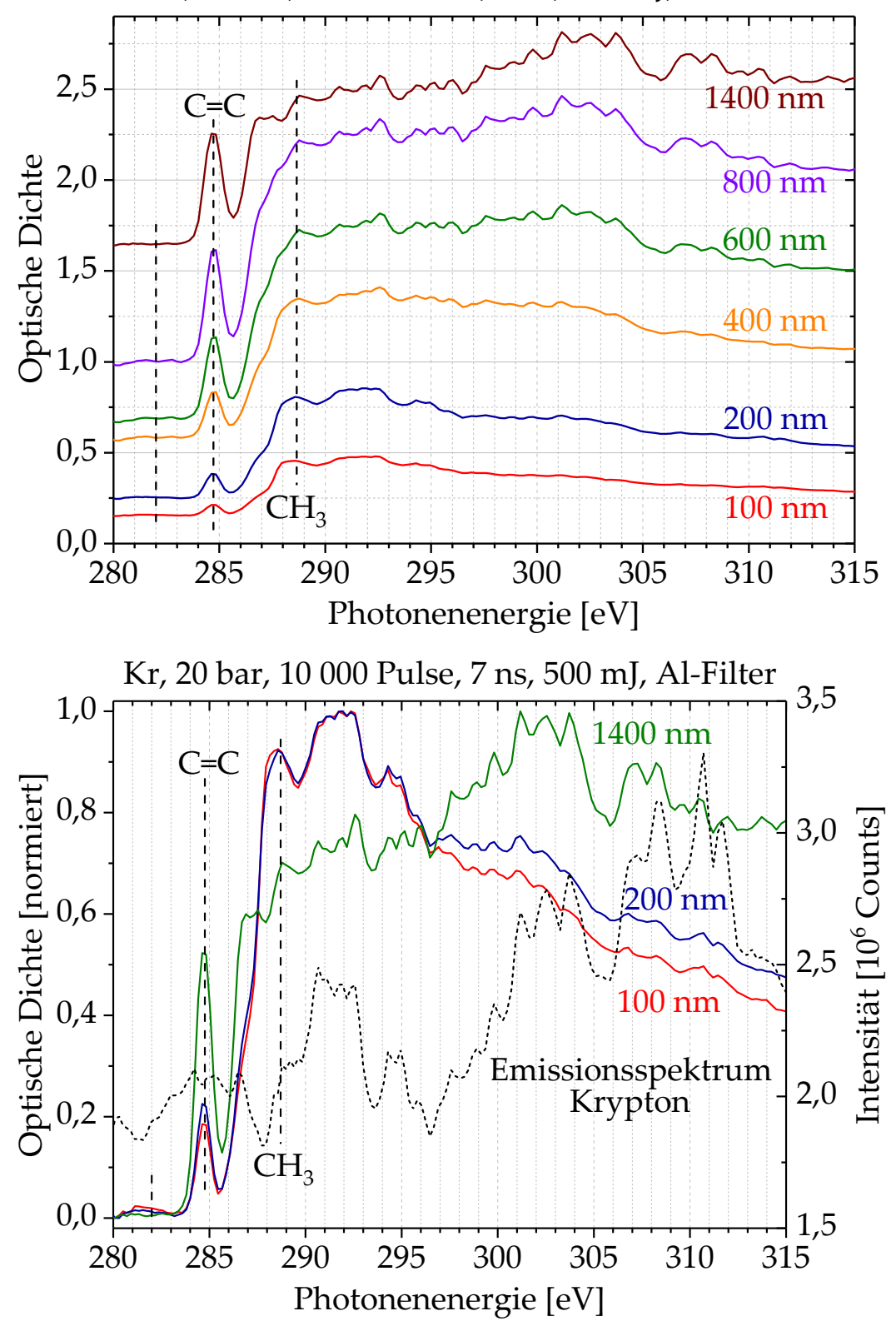

Abbildung 4.13: NEXAFS-Spektren von Agar-100 (Epoxidharz) verschiedener Probendicke an der Kohlenstoff-K-Kante. Um qualitative Unterschiede in den Spektren zu veranschaulichen, sind unten die normierten Spektren der 100, 200 und $1400 \mathrm{~nm}$ dicken Proben aufgetragen. Zusätzlich ist das Emissionsspektrum von Krypton aufgetragen. 

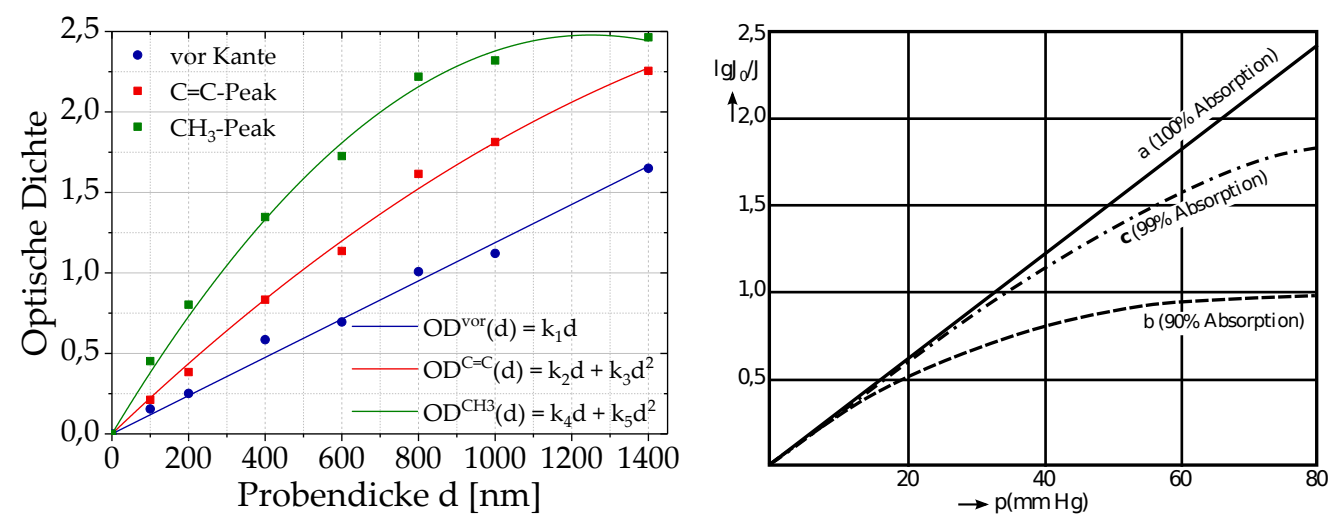

Abbildung 4.14: Optische Dichte der Agar-100-Proben vor der Kohlenstoff-KKante $(282 \mathrm{eV})$, am C=C-Peak $(284,7 \mathrm{eV})$ sowie $\mathrm{CH}_{3}$-Peak $(288,7 \mathrm{eV})$ aufgetragen gegen die Probendicke (links) und Abhängigkeit der optischen Dichte von der Probendicke bei "unvollständiger Absorption“ (rechts, übernommen aus [241]). Die Parameter $k_{1}$ bis $k_{5}$ sind in Tabelle A.8 aufgelistet.

den. Die Position beider Peaks stimmt für alle Proben jedoch bis auf eine minimale Abweichung von 0,1 eV mit Synchrotrondaten überein [240].

Um die Unterschiede in den Spektren quantitativ zu erfassen, wurden die optischen Dichten vor der Kohlenstoff-K-Kante bei $282 \mathrm{eV}$ sowie der $\mathrm{C}=\mathrm{C}$ - und $\mathrm{CH}_{3}$-Peaks gegen die Probendicke aufgetragen (siehe Abb. 4.14). Gemäß dem Lambert-Beer'schen Gesetz (siehe Gl. (4.I)) ist die optische Dichte direkt proportional zur Schichtdicke d, wobei der konstante Proportionalitätsfaktor dem linearen Absorptionskoeffizient $\mu$ entspricht. Die Gültigkeit des Lambert-Beer'schen Gesetzes kann demnach überprüft werden, indem an die in Abbildung 4.14 aufgetragenen Daten eine Ursprungsgerade angepasst wird. Die optische Dichte vor der Kohlenstoff-K-Kante folgt diesem linearen Zusammenhang. Um die Messpunkte des $\mathrm{C}=\mathrm{C}$ - und $\mathrm{CH}_{3}$-Peaks zu beschreiben, ist hingegen ein zusätzlicher quadratischer Term für die Schichtdicke notwendig. Die optische Dichte der energetisch höher gelegenen $\mathrm{CH}_{3}$-Bindung weicht dabei mit zunehmenden Probendicke stärker von der Ursprungsgeraden $a b$ als die der $C=C$-Doppelbindung. Ursache für dieses Verhalten ist der Effekt der „unvollständigen Absorption“5: Ist der Absorptionskoeffizient innerhalb eines Energiebereichs endlicher Breite nahezu konstant (vor der Absorptionskante), dann wird die Röntgenstrahlung in gleichen Schichten im gleichen Verhältnis geschwächt und die optische Dichte steigt linear an. An der Kante ändert sich der Absorptionskoeffizient hingegen sprunghaft; der Anstieg ist allerdings endlich (siehe Abb. 4.2). Die Absorption variiert demnach innerhalb des Energiebereichs und mit zunehmender Schichtdicke ist keine vollständige Absorption zu errei-

5 Bereits 1905 hat Clemens Schaefer die „unvollständige Absorption“ im Infrarotbereich bei Absorptionsmessungen für Kohlensäure beobachtet und gefolgert, dass die Absorptionsbanden eine Feinstruktur aufweisen müssen [242]. 
chen. Der Absorptionskoeffizient hängt von der Probendicke ab und das Lambert-Beer'sche Gesetz wird ungültig. In Abbildung 4.14 rechts ist dieser Effekt für vollständige (Kurve a) und unvollständige Absorption von $99 \%$ bzw. $90 \%$ (Kurve b und c) gezeigt [241].

Ein Maß, um die geeignete Probendicke zu ermitteln, ist die Eindringtiefe $d_{\mathrm{e}}$. Sie folgt aus dem Lambert-Beer'schen Gesetz (siehe Gl. (4.I)) und gibt an, nach welcher Wegstrecke die Intensität der einfallenden Röntgenstrahlung aufgrund von Absorption auf das 1/e-fache (etwa $37 \%$ ) ihres ursprünglichen Wertes $\mathrm{I}_{0}$ abgesunken ist. Die optische Dichte beträgt in diesem Fall 1. Die Eindringtiefe kann als Funktion der Photonenenergie z. B. aus der CXRO-Datenbank [120] entnommen werden. Für aromatische Kohlenstoffverbindungen wie die untersuchten Agar100-Proben liegt die Eindringtiefe an der Kohlenstoff K-Kante im Bereich von 200 bis $300 \mathrm{~nm}$. Dieser Wert stimmt sehr gut mit dem annähernd linearen Verlauf der optischen Dichte des $\mathrm{CH}_{3}$-Peaks für Probendicken kleiner $(250 \pm 50) \mathrm{nm}$ überein (siehe Abb. 4.14). Um eine vollständige Absorption und die Gültigkeit des Lambert-Beer'schen Gesetzes zu gewährleisten, sollten für NEXAFS-Messungen folglich Proben mit einer Dicke unterhalb der jeweiligen Eindringtiefe verwendet werden.

\subsubsection{EISENMINERALE UND SILIKATE}

Die Eisen-L-Kante liegt je nach Material bei Photonenenergien zwischen 705 und $730 \mathrm{eV}$. Daher eignen sich Proben verschiedener Eisenminerale wie Hämatit, Goethit und Ferrihydrit, um das Leistungsvermögen des verbesserten Spektrometers (siehe Abs. 4.4) für die Aufnahme von NEXAFS-Spektren an und oberhalb der Sauerstoff-K-Kante (Energie > $530 \mathrm{eV}) \mathrm{zu}$ testen.

Zuerst wurden Hämatit-Proben $\left(\mathrm{Fe}_{2} \mathrm{O}_{3}\right)$ mit Dicken zwischen 25 und $85 \mathrm{~nm}$ analysiert, die von Ahmed Ismail (Arbeitsgruppe Prof. Dr. Frank de Groot, Universität Utrecht) mittels Sprühpyrolyse aus geeigneten Ausgangsverbindungen hergestellt wurden. Dieses Verfahren bietet den Vorteil, dass die Probendicke bis auf wenige Nanometer genau kontrolliert werden kann und die Proben zudem eine sehr homogene Verteilung auf der $\mathrm{Si}_{3} \mathrm{~N}_{4}$-Membran aufweisen. In Abbildung 4.15 sind die NEXAFSSpektren der Hämatit-Proben gezeigt. Selbst für die dünnste Probe mit einer Dicke von lediglich $25 \mathrm{~nm}$ weisen die Spektren ausgeprägte Merkmale an der Sauerstoff-K- und Eisen-L,2-Kante auf. Dieses Ergebnis unterstreicht, dass die hohe Sensitivität der NEXAFS-Spektroskopie auch mit dem Laborgerät erreicht wird. Die sehr gute Sichtbarkeit der Kanten lässt vermuten, dass auch geringere Dicken messbar wären. Die minimale Probendicke kann in zukünftigen Messungen im Rahmen der Kooperation mit der Arbeitsgruppe de Groot bestimmt werden. 


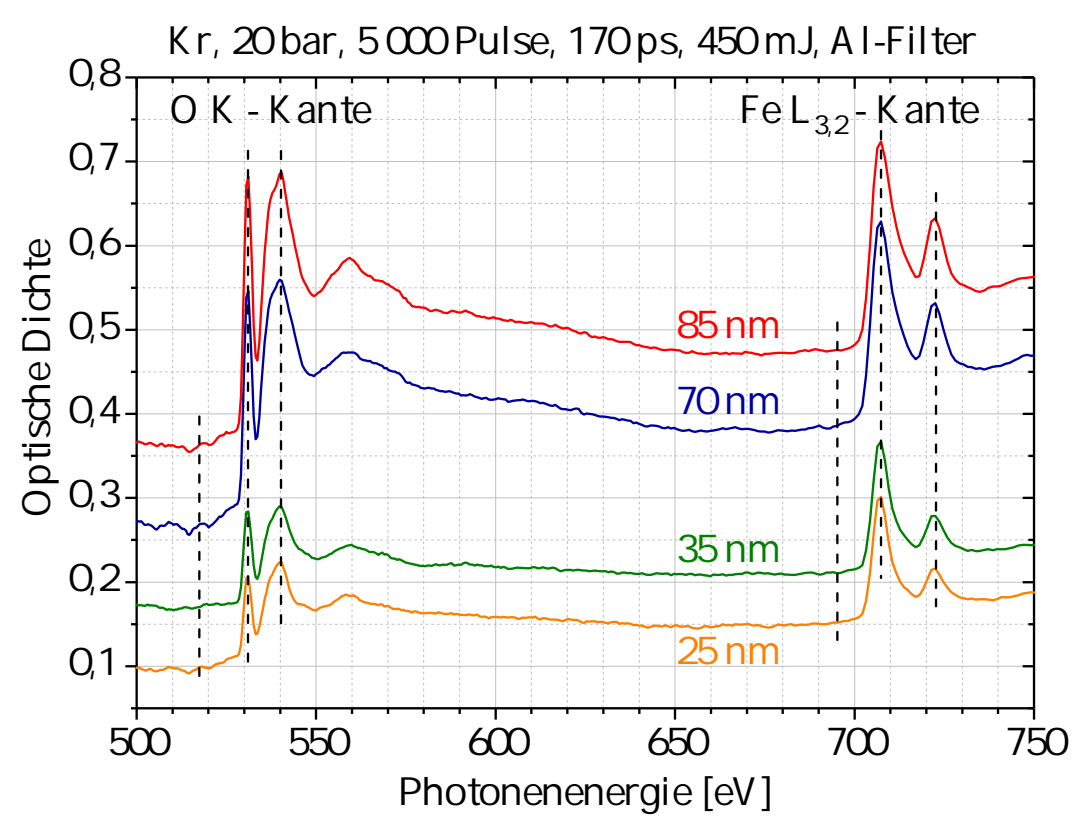

Abbildung 4.15: NEXAFS-Spektren von Hämatit verschiedener Probendicken an der Sauerstoff-K- und Eisen- $\mathrm{L}_{3,2}$-Kante. Die Proben wurden mittels Sprühpyrolyse von Ahmed Ismail (Arbeitsgruppe Prof. Dr. Frank de Groot, Universität Utrecht) hergestellt.

Die Eindringtiefe für Hämatit beträgt für die Sauerstoffkante etwa $140 \mathrm{~nm}$ und für die Eisenkante ungefähr $80 \mathrm{~nm}$ [120]. Die Gültigkeit des Lambert-Beer'schen Gesetzes sollte demnach gegeben sein. In der Tat kann die optische Dichte an beiden Kanten als Funktion der Probendicke durch eine Ursprungsgerade beschrieben werden (siehe Abb. 4.16). Ähnlich wie für Agar-100 wurde dazu die optische Dichte für alle vier Hämatit-Proben für eine Photonenenergie vor und an den beiden charakteristischen Peaks nach der Absorptionskante ermittelt.
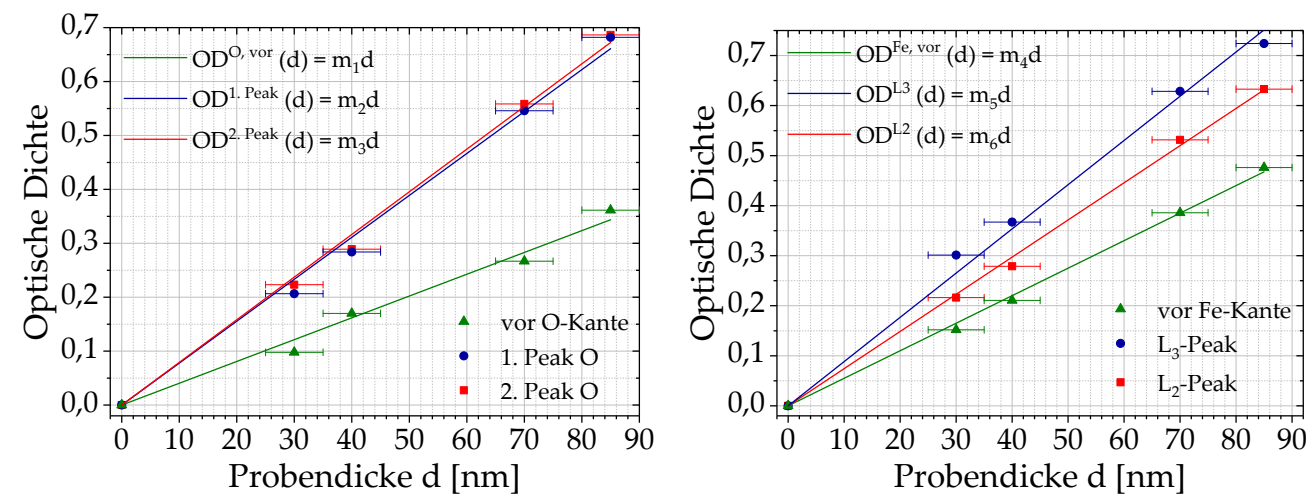

Abbildung 4.16: Optische Dichte der Hämatit-Proben für ausgewählte Photonenenergien an der Sauerstoff-K- (links) und Eisen-L,2-Kante (rechts) aufgetragen gegen die Probendicke (Messunsicherheit $\pm 5 \mathrm{~nm}$ ). Die Parameter $m_{1}$ bis $m_{6}$ sind in Tabelle A.9 aufgelistet. 
In den beiden folgenden Abschnitten werden NEXAFS-Spektren weiterer Eisenminerale (Hämatit, Goethit, Ferrihydrit) und des Schichtsilikats CCa-2 Chlorit an der Sauerstoff-K- und Eisen-L $\mathrm{L}_{3,2}$-Kante gezeigt. Das Ausgangsmaterial für die Proben wurde als wässrige Lösung von Karin Eusterhues (Friedrich-Schiller-Universität Jena) bereitgestellt. Um möglichst homogene Schichten messbarer Dicke zu präparieren, wurden die Lösungen zunächst im Verhältnis 1 : 10 mit Wasser dispergiert und anschließend auf einer $100 \mathrm{~nm}$ dünnen $\mathrm{Si}_{3} \mathrm{~N}_{4}$-Membran aufgetragen. In einigen Fällen formte das Material auf der Probe während des Trocknens größere Aggregate. Daher mussten teils mehrere Versuche unternommen werden, um geeignete Proben zu erhalten.

Um die Absorptionsspektren interpretieren zu können, ist es hilfreich, die elektronische Struktur der untersuchten Proben zu betrachten. In Abbildung 4.17 ist beispielhaft das vereinfachte Termschema von Hämatit und Goethit dargestellt [243]: Das unterste unbesetzte Energieniveau in der Konfiguration „Spin down“ entspricht einem Fe(3d)-Niveau. Ener-
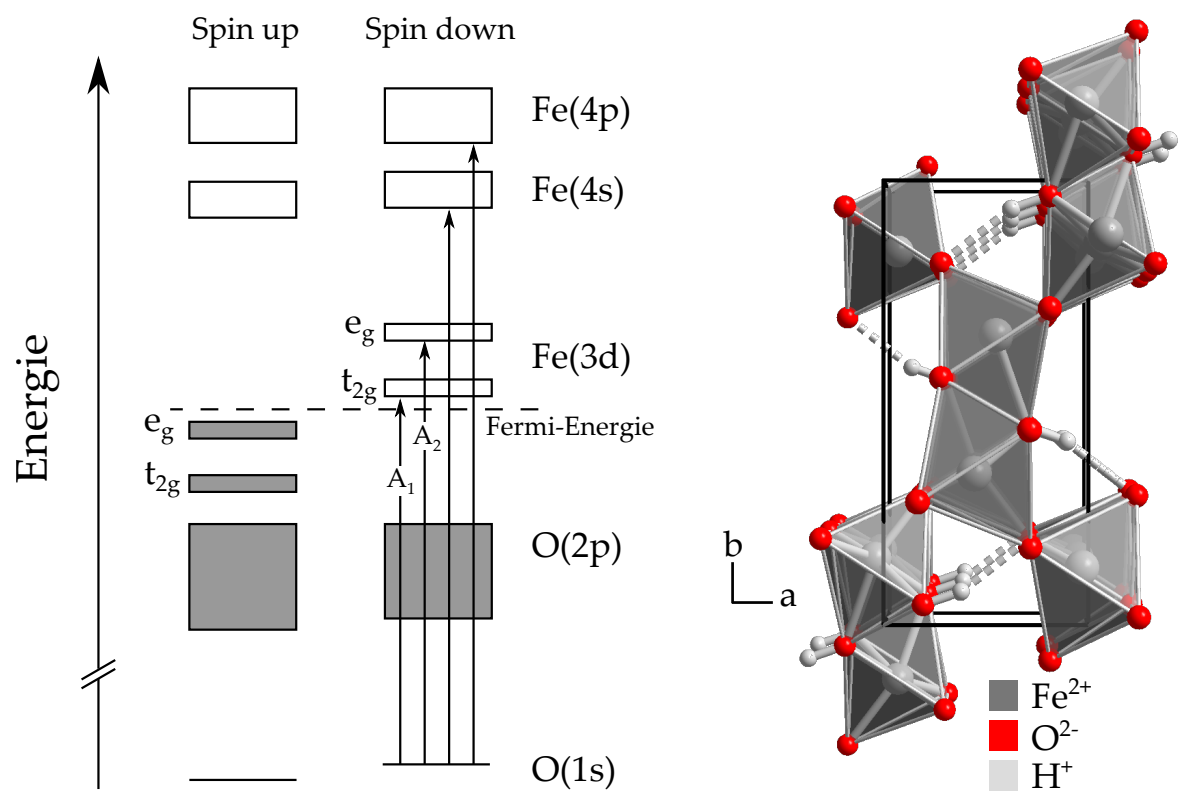

Abbildung 4.17: Vereinfachtes Termschema (links) von Eisenoxiden (z. B. Goethit und Hämatit, übernommen aus Ref. [243]) sowie Kristallstruktur (rechts) von Goethit [244].

getisch höher gelegen sind die $\mathrm{Fe}(4 \mathrm{~s})$ - und $\mathrm{Fe}(4 \mathrm{p})$-Orbitale. Alle drei bilden dabei mit dem $\mathrm{O}(2 \mathrm{p})$-Orbital kombinierte Hybridorbitale. Für Goethit gibt es zusätzlich zum $\mathrm{O}(1 \mathrm{~s})$ - ein $\mathrm{OH}(1 \mathrm{~s})$-Niveau (nicht gezeigt in Abb. 4.17), aus dem Elektronen in unbesetzte Orbitale angeregt werden können. Die unterschiedlichen erlaubten elektronischen Übergänge zwischen den Niveaus führen im NEXAFS-Spektrum zu charakteristischen Peaks an den Absorptionskanten von Sauerstoff und Eisen, die im Folgenden diskutiert werden. 


\section{SAUERSTOFF-K-KANTE}

In Abbildung 4.18 sind die NEXAFS-Spektren der drei Eisenminerale Hämatit, Goethit, Ferrihydrit und des Silikatminerals CCa-2 Chlorit an der Sauerstoff-K-Kante gezeigt. Der Verlauf der optischen Dichte stimmt sehr gut mit Daten überein, die am Synchrotron von Brandt et al. [245] gemessen wurden. Die drei charakteristischen Merkmale A („pre-edge“), B („main edge") und C (,post-edge") lassen sich eindeutig zuordnen. Peak A repräsentiert dabei den Übergang vom $\mathrm{O}(1 \mathrm{~s})$ - in das $\mathrm{Fe}(3 \mathrm{~d})$ Orbital und Peak B die Anregung aus dem O(1s)- in die Fe(4s, 4p)Niveaus [243]. Ebenso zeigt CCa-2 Chlorit anders als Hämatit, Goethit und Ferrihydrit keine bzw. nur eine sehr schwach ausgeprägte „preedge" A. Die spektrale Auflösung hingegen ist am Synchrotron höher: Statt eines breiten Peaks $A$ sind somit zwei Peaks $A_{1}$ und $A_{2}$ deutlich sichtbar. Diese Aufspaltung korreliert mit der oktaedrischen Anordnung der Sauerstoffmoleküle um das Eisen (siehe Abb. 4.17), die zu einer Aufhebung der Entartung der 3d-Orbitale im so entstehenden Ligandenfeld führt. Die Energiedifferenz zwischen dem $t_{2 g^{-}}$und $e_{g}$-Orbital ist ein direktes Maß für die Stärke des Ligandenfeldes [245]. Das Fehlen der „preedge" in CCa-2 Chlorit wiederum deutet auf eine verzerrte oktaedrische Koordination hin [246]. Aufgrund des zusätzlichen $\mathrm{OH}(1 \mathrm{~s})$-Orbitals in Goethit und damit verbunden einem weiteren Übergang zwischen den Molekülorbitalen ergibt sich in dessen Spektrum ein dritter Peak $A_{3}$.

Kr, 20 bar, 170 ps, 450 mJ, Al-Filter
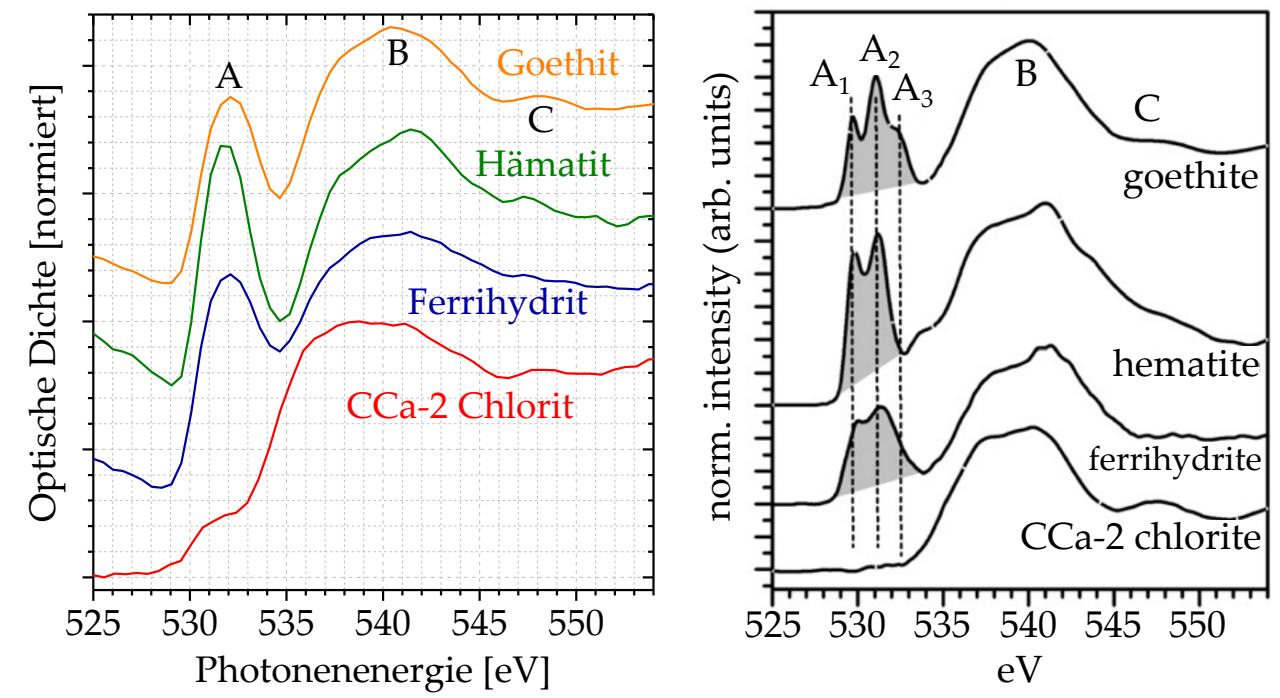

Abbildung 4.18: NEXAFS-Spektren dreier verschiedener Eisenminerale und des Schichtsilikats CCa-2 Chlorit an der Sauerstoff-K-Kante, gemessen mit dem Laborröntgenspektrometer (links) und am Synchrotron (rechts, übernommen aus [245]). CCa-2 Chlorit (20000 Pulse) wurde vor, Ferrihydrit, Hämatit und Goethit (1000 Pulse) wurden nach der Optimierung des Aufbaus gemessen. Zur besseren Übersicht sind die Spektren entlang der $y$-Achse verschoben. 

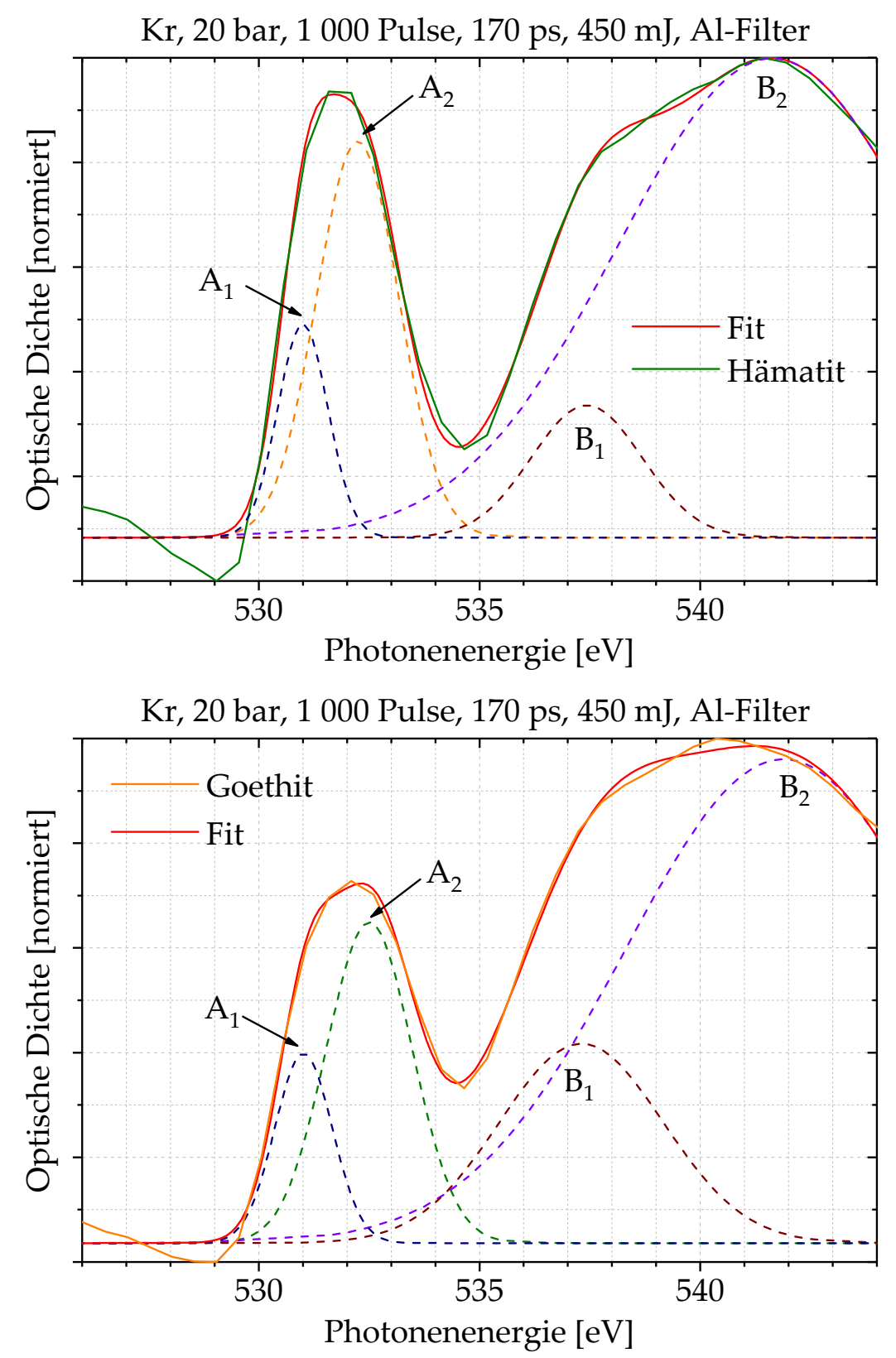

Abbildung 4.19: NEXAFS-Spektrum von Hämatit (oben) und Goethit (unten) an der Sauerstoff-K-Kante sowie daran angepasste Fitfunktion bestehend aus vier Gaußkurven. Die Fitparameter sind in Tabelle A.1o aufgelistet.

Obwohl die Auflösung des Laborröntgenspektrometers geringer ist als an Synchrotronen, lassen sich aus den Hämatit- und Goethitspektren die Merkmale $A_{1}$ und $A_{2}$ sowie $B$ durch Anpassen von Gaußkurven an die optische Dichte extrahieren (siehe Abb. 4.19). In Tabelle 4.I sind die Energiepositionen der vier Peaks im Vergleich zu Synchrotrondaten zusammengefasst. Die Messdaten stimmen sehr gut mit den Werten überein, die von Todd et al. [243] mittels ",total electron yield“ bestimmt wurden. Die maximale Abweichung beträgt lediglich 0,33\%. Auch die Energiedifferenz als Maß für die Stärke des Ligandenfeldes zwischen 
den Peaks $A_{1}$ und $A_{2}$ von 1,2 eV für Hämatit bzw. 1,5 eV für Goethit ist konsistent zu Literaturwerten, die im Bereich von 1,2 bis 1,4 eV [243, 245,247 ] liegen. Einzig der Peak $A_{3}$ der Goethit-Probe kann wegen der zu geringen spektralen Auflösung nicht gemessen werden.

Tabelle 4.1: Energie der vier durch Gaußfit ermittelten Peaks an der SauerstoffK-Kante von Hämatit sowie Goethit, die dazugehörigen elektronischen Übergänge und prozentuale Abweichung $\Delta \mathrm{zu}$ Vergleichsdaten [243]

\begin{tabular}{|c|c|c|c|c|c|}
\hline & Übergang & Peak & Energie $[\mathrm{eV}]$ & $\begin{array}{l}\text { Literatur- } \\
\text { wert }[\mathrm{eV}]\end{array}$ & $\Delta[\%]$ \\
\hline \multirow{4}{*}{ 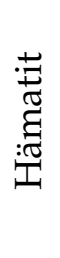 } & $\mathrm{O}(\mathrm{1s}) \rightarrow \mathrm{t}_{2 \mathrm{~g}}-\mathrm{Fe}(3 \mathrm{~d})$ & $\mathrm{A}_{1}$ & $531,0(3)$ & 529,0 & 0,23 \\
\hline & $\mathrm{O}(\mathrm{Is}) \rightarrow \mathrm{e}_{\mathrm{g}}-\mathrm{Fe}(3 \mathrm{~d})$ & $\mathrm{A}_{2}$ & $532,2(5)$ & 531,2 & 0,23 \\
\hline & $\mathrm{O} 1 \mathrm{~s}) \rightarrow \mathrm{Fe}(4 \mathrm{~s}, 4 \mathrm{p})$ & $\mathrm{B}_{1}$ & $537,4(2)$ & 539,0 & 0,31 \\
\hline & $\mathrm{O}(\mathrm{Is}) \rightarrow \mathrm{Fe}(4 \mathrm{~s}, 4 \mathrm{p})$ & $\mathrm{B}_{2}$ & $541,6(4)$ & 542,0 & 0,09 \\
\hline \multirow{5}{*}{ 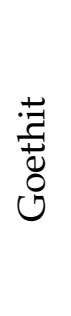 } & $\mathrm{O}(1 \mathrm{~s}) \rightarrow \mathrm{t}_{2 \mathrm{~g}}-\mathrm{Fe}(3 \mathrm{~d})$ & $\mathrm{A}_{1}$ & $531,0(2)$ & 529,0 & 0,23 \\
\hline & $\mathrm{O}(1 \mathrm{~s}) \rightarrow \mathrm{e}_{\mathrm{g}}-\mathrm{Fe}(3 \mathrm{~d})$ & $\mathrm{A}_{2}$ & $532,5(3)$ & 531,2 & 0,25 \\
\hline & $\mathrm{OH}(1 \mathrm{~s}) \rightarrow \mathrm{e}_{\mathrm{g}}-\mathrm{Fe}(3 \mathrm{~d})$ & $\mathrm{A}_{3}$ & - & 532,6 & - \\
\hline & $\mathrm{O}(1 \mathrm{~s}) \rightarrow \mathrm{Fe}(4 \mathrm{~s}, 4 \mathrm{p})$ & $\mathrm{B}_{1}$ & $537,3(2)$ & 539,0 & 0,33 \\
\hline & $\mathrm{O}(1 \mathrm{~s}) \rightarrow \mathrm{Fe}(4 \mathrm{~s}, 4 \mathrm{p})$ & $\mathrm{B}_{2}$ & $541,9(12)$ & 542,0 & 0,03 \\
\hline
\end{tabular}

EISEN-L 3,2-KANTE

Die NEXAFS-Spektren von Hämatit, Ferrihydrit und CCa-2 Chlorit an der Eisen-L,2-Kante sind in Abbildung 4.20 dargestellt. Die $\mathrm{L}_{3}$ - und $\mathrm{L}_{2}$-Peaks, die aus Übergängen vom $\mathrm{Fe}(2 \mathrm{p})$-Orbital in leere $\mathrm{t}_{2 \mathrm{~g}^{-}}$und $\mathrm{e}_{g^{-}}$$\mathrm{Fe}(3 \mathrm{~d})$-Orbitale und in $\mathrm{Fe}(4 \mathrm{~s})$-ähnliche Niveaus resultieren, lassen sich eindeutig zuordnen. Die Spektren stimmen bezüglich der Peakposition mit einer Abweichung von maximal 0,35\% zudem sehr gut mit Synchrotrondaten von Brandt et al. [245] überein (siehe Tab. 4.2). Dabei konnte die Messzeit von über einer Stunde im alten Aufbau auf weniger als fünf Minuten im optimierten Aufbau verkürzt werden. Die Aufspaltung der $\mathrm{L}_{3}$ - und $\mathrm{L}_{2}$-Peaks kann aufgrund der geringeren Auflösung des Laborröntgenspektrometers allerdings nicht gemessen werden.

Im Gegensatz zur Sauerstoffkante ist die Energiedifferenz zwischen $\mathrm{t}_{2 \mathrm{~g}}$ - und $\mathrm{e}_{\mathrm{g}}$-Peak aufgrund der stärkeren Relaxation während des $\mathrm{Fe}(2 \mathrm{p})$ $\rightarrow \mathrm{Fe}(3 \mathrm{~d})$-Übergangs größer (1,7 eV statt 1,2 eV für Hämatit) und ist kein direktes Maß für die Stärke des Ligandenfeldes [243]. Allerdings spiegelt sich im Verhältnis der optischen Dichten beider Peaks der Anteil von $\mathrm{Fe}^{2+} \mathrm{zu} \mathrm{Fe}^{3+}$ wider: Je intensiver der $\mathrm{t}_{2 \mathrm{~g}}$-Peak ausgeprägt ist, desto höher ist der Anteil an $\mathrm{Fe}^{2+}$. Zu erwarten ist daher, dass das Spektrum 
Kr, 20 bar, 170 ps, 450 mJ, Al-Filter
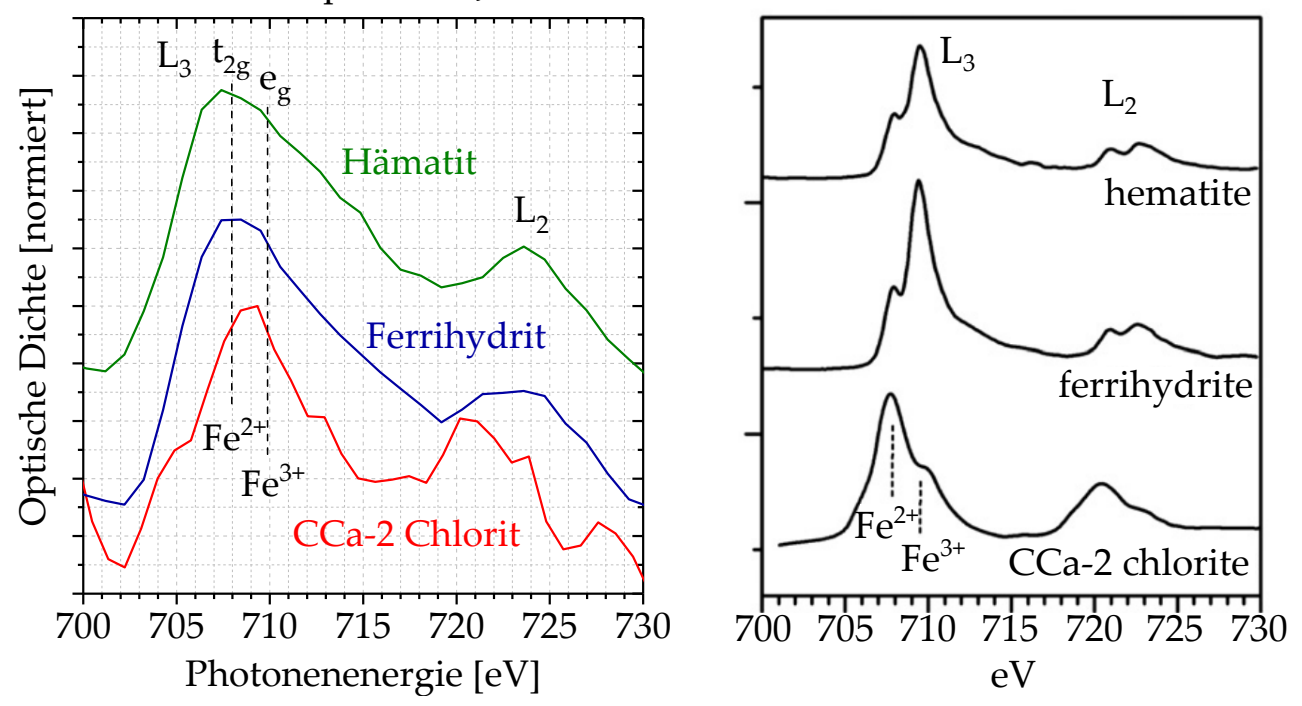

Abbildung 4.20: NEXAFS-Spektren unterschiedlicher Eisenminerale und des Schichtsilikats CCa-2 Chlorit an der Eisen-L $\mathrm{L}_{3,2}$-Kante, gemessen mit Laborröntgenspektrometer (links) und am Synchrotron (rechts, übernommen aus [245]). CCa-2 Chlorit (20000 Pulse) wurde vor, Ferrihydrit und Hämatit (1000 Pulse) wurden nach der Optimierung des Aufbaus gemessen. Zur besseren Übersicht sind die Spektren entlang der $y$-Achse verschoben.

von CCa-2 Chlorit einen starken $t_{2 g}$-Peak aufweist, während bei Hämatit und Ferrihydrit aufgrund des $\mathrm{Fe}^{3+}$ in oktaedrischer Koordination der $\mathrm{e}_{\mathrm{g}}$-Peak überwiegt. Dieser Unterschied in den NEXAFS-Spektren ist aber nur in den Synchrotrondaten sichtbar.

Auch das Verhältnis der optischen Dichte von $\mathrm{L}_{3}$ - und $\mathrm{L}_{2}$-Peak gibt Aufschluss über den Anteil der verschiedenen Eisenkonfigurationen in der Probe [248]: Für $\mathrm{Fe}^{2+}$ ist es geringer als für $\mathrm{Fe}^{3+}$. Tatsächlich ist das $\mathrm{L}_{3} / \mathrm{L}_{2}$-Verhältnis für CCa-2 Chlorit mit 1,55 signifikant kleiner als für Hämatit und Ferrihydrit mit 2,23 bzw. 2,46. Somit lassen sich trotz der verminderten spektralen Auflösung des Laborröntgenspektrometers zumindest qualitative Unterschiede zwischen den Eisen nachweisen. Zu beachten ist dabei, dass Eisen gleicher Oxidationsstufe - wie in Hämatit, Goethit und Ferrihydrit - selbst in Messungen am Synchrotron an der Eisen-L $\mathrm{L}_{3,2}$-Kante quasi ununterscheidbar sind [245]. Die Differenzierung erfolgt daher meist anhand der Sauerstoff-K-Kante.

In Abbildung 4.21 ist das NEXAFS-Spektrum des Eisen(II)disulfids Pyrit $\left(\mathrm{FeS}_{2}\right)$ an der Eisen-L $\mathrm{L}_{3,2}$-Kante gezeigt. Erneut ist bis auf die verminderte spektrale Auflösung die sehr gute Übereinstimmung mit Synchrotrondaten [243] offensichtlich. Da das Spektrum lediglich aus Übergängen von $\mathrm{Fe}(2 \mathrm{p})$-Niveaus in das leere $\mathrm{e}_{\mathrm{g}} \mathrm{Fe}(3 \mathrm{~d})$-Orbital resultiert, erfolgt in reinem Pyrit keine weitere Aufspaltung des $\mathrm{L}_{3}$ - und $\mathrm{L}_{2}$-Peaks. Allerdings oxidiert das Eisen der Pyrit-Probe, sodass an der Probenoberfläche neben $\mathrm{Fe}^{(2+)}$ eine geringe Menge $\mathrm{Fe}^{(3+)}$ entsteht. Daher ist in 
den Spektren von Todd et al. [243] dennoch eine kleine Aufspaltung zu erkennen. Das Verhältnis $L_{3} / L_{2}$ beträgt für Pyrit 1,28 und ist, wie für $\mathrm{Fe}^{(2+)} \mathrm{zu}$ erwarten, viel geringer als z. B. für Hämatit und Ferrihydrit.

Tabelle 4.2: Energie der Peaks L3,2 von Hämatit, Ferrihydrit, CCa-2 Chlorit sowie Pyrit und prozentuale Abweichung $\Delta \mathrm{zu}$ Vergleichsdaten $[243,245]$

\begin{tabular}{|c|c|c|c|c|}
\hline & Peak & Energie [eV] & $\begin{array}{l}\text { Literatur- } \\
\text { wert }[\mathrm{eV}]\end{array}$ & $\Delta[\%]$ \\
\hline \multirow{2}{*}{ 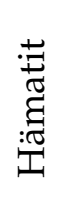 } & $\mathrm{L}_{3}$ & $707,4(5)$ & $\begin{array}{l}708,1\left(\mathrm{Fe}^{2+}\right) \\
709,8\left(\mathrm{Fe}^{3+}\right)\end{array}$ & $\begin{array}{l}0,10 \\
0,33\end{array}$ \\
\hline & $\mathrm{L}_{2}$ & $723,6(5)$ & $\begin{array}{l}721,1 \\
723,0\end{array}$ & $\begin{array}{l}0,35 \\
0,09\end{array}$ \\
\hline \multirow{2}{*}{ 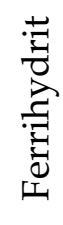 } & $\mathrm{L}_{3}$ & $707,4(5)$ & $\begin{array}{l}708,1\left(\mathrm{Fe}^{2+}\right) \\
709,8\left(\mathrm{Fe}^{3+}\right)\end{array}$ & $\begin{array}{l}0,10 \\
0,33\end{array}$ \\
\hline & $\mathrm{L}_{2}$ & $723,6(5)$ & $\begin{array}{l}721,1 \\
723,0\end{array}$ & $\begin{array}{l}0,35 \\
0,09\end{array}$ \\
\hline \multirow{2}{*}{$\bigcup_{U}^{N}$} & $\mathrm{~L}_{3}$ & $709,3(5)$ & $\begin{array}{l}707,8\left(\mathrm{Fe}^{2+}\right) \\
709,5\left(\mathrm{Fe}^{3+}\right)\end{array}$ & $\begin{array}{l}0,22 \\
0,04\end{array}$ \\
\hline & $\mathrm{L}_{2}$ & $\begin{array}{l}720,2(5) \\
723,9(5)\end{array}$ & $\begin{array}{l}720,8 \\
723,0\end{array}$ & $\begin{array}{l}0,09 \\
0,13\end{array}$ \\
\hline \multirow{2}{*}{ 孛 } & $\mathrm{L}_{3}$ & $709,3(5)$ & $709,5\left(\mathrm{Fe}^{2+}\right)$ & 0,03 \\
\hline & $\mathrm{L}_{2}$ & $\begin{array}{l}722,0(5) \\
723,9(5)\end{array}$ & $\begin{array}{l}721,8 \\
723,3\end{array}$ & $\begin{array}{l}0,03 \\
0,08\end{array}$ \\
\hline
\end{tabular}

ERHÖHUNG DES AUFLÖSUNGSVERMÖGENS

Die spektrale Auflösung des Laborröntgenspektrometers ist bisher nicht ausreichend, um alle charakteristischen Merkmale in den Spektren der eisenhaltigen Minerale zu bestimmen. Im Gegensatz zur Sauerstoff-KKante (siehe Abb. 4.19) ließen sich keine weiteren Informationen aus der optischen Dichte an der Eisen-L,2-Kante durch das Anpassen von Gaußkurven gewinnen. Daher muss die Auflösung des Spektrometers erhöht werden, um die Feinstruktur der Peaks messen zu können. Aufgrund der Lebensdauer des unbesetzten Elektronenzustands („,core hole“) beträgt die Breite der Peaks an der Eisen-L,2-Kante etwa 0,4 bis 0,5 eV [249]. Bei einer Energie von $E \approx 700 \mathrm{eV}$ ist somit eine Auflösung größer $\mathrm{E} / \Delta \mathrm{E}=1400$ anzustreben. Gegenwärtig ist das theoretische Auflösungsvermögen jedoch bedingt durch die Parameter des Reflexionsgitters um einen Faktor 3 bis $4 \mathrm{zu}$ klein. Es wird insbesondere aufgrund des geringen Abstands (ca. 235 mm) zwischen Gitter und Kamera begrenzt. Daher soll für den Aufbau eines hochauflösenden Röntgenspektrometers 

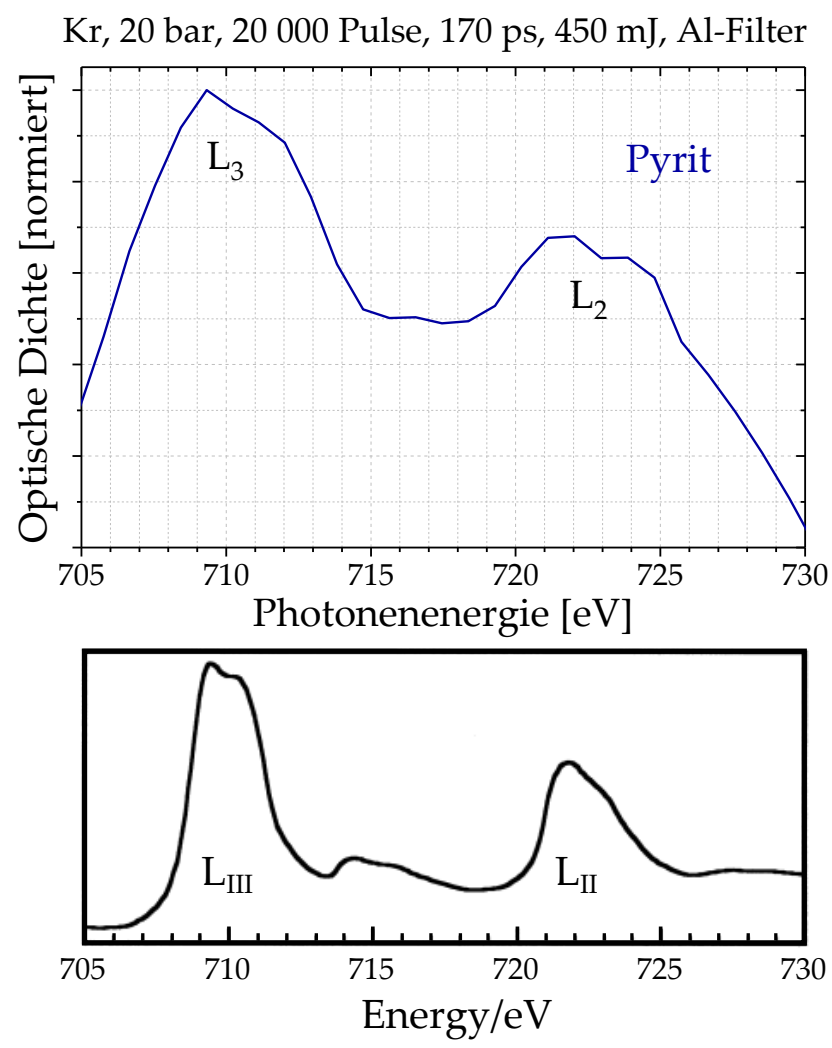

Abbildung 4.21: NEXAFS-Spektrum von Pyrit an der Eisen-L $\mathrm{L}_{3,2}$-Kante, gemessen mit Laborröntgenspektrometer (oben) und am Synchrotron (unten, übernommen aus Ref. [243]) 
ein optimiertes Reflexionsgitter ${ }^{6}$ eingesetzt werden, bei dem dieser $\mathrm{Ab}$ stand um das 2,4fache auf etwa $565 \mathrm{~mm}$ vergrößert wird. Zudem wird die Gitterkonstante um den Faktor 1,5 auf 3600 Linien/mm erhöht.

Alternativ könnten Transmissionsgitter eingesetzt werden, für die Gitterkonstanten von bis zu 5000 Linien/mm möglich sind. Jedoch ist die Dispersion eines Gitters in Transmission nach Gleichung (4.3) aufgrund des Einfallswinkels von $\alpha=0^{\circ}$ geringer als in Reflexion. Um mit einem Transmissionsgitter die gleiche spektrale Auflösung zu erreichen, müsste die Optik zur Fokussierung der Röntgenstrahlung daher eine etwa 5 fach größere Brennweite besitzen als das neu konzipierte Gitter. Darüber hinaus sind Transmissionsgitter mechanisch instabiler. Insbesondere wenn der Aufbau regelmäßig be- und entlüftet werden muss (etwa für den Probenwechsel), besteht ein großes Risiko, das Gitter zu beschädigen. Angesichts dieser Nachteile wird auf den Einsatz eines Transmissionsgitters verzichtet.

\subsection{AUSBLENDEN DER 0. ORDNUNG}

Durch Verkürzen der Abstände zwischen Quelle, Probe und Eintrittsspalt konnte das Signal-Rausch-Verhältnis in den Spektren erhöht werden (siehe Abs. 4.4). Gleichzeitig ist im Emissionsspektrum von Krypton $\mathrm{zu}$ sehen, dass die Intensität nach Erreichen des Maximums bei ca. $400 \mathrm{eV}$ ab etwa $750 \mathrm{eV}$ erneut leicht ansteigt (siehe Abb. 4.22). Diese Zunahme ist auf Licht aus der 0. Beugungsordnung zurückzuführen, deren Ausläufer sich mit der 1. Beugungsordnung auf dem CCD-Chip überlagern. Mit einer zusätzlichen Blende direkt hinter dem Gitter lässt sich dieser Anteil ausblenden. Um die Blende möglichst genau im Strahlengang auszurichten, wurde die optische Dichte einer $100 \mathrm{~nm}$ dicken $\mathrm{Si}_{3} \mathrm{~N}_{4}$-Membran für verschiedene Blendenpositionen gemessen und mit den Daten der CXRO-Datenbank [120] verglichen. In Abbildung 4.22 ist die optische Dichte an der Stickstoff-K-Kante aus der Messung ohne Blende und für die Position der Blende mit der besten Übereinstimmung zu den Literaturwerten aufgetragen. Das Ausblenden der 0. Beugungsordnung führt demnach dazu, dass die optische Dichte nicht nur qualitativ (Position der Absorptionskante), sondern auch quantitativ mit Referenzdaten übereinstimmt.

Daran anschließend wurde der Einfluss der Blende bei höheren Photonenenergien untersucht, indem erneut Spektren der beiden Eisenoxide Goethit und Ferrihydrit aufgenommen wurden (siehe Abb. 4.23). Für beide Proben ist die an den zwei Absorptionskanten mit Blende gemessene optische Dichte geringer als die ohne Blende gemessene. Der Verlauf entspricht damit deutlich besser den Erwartungen: Die optische Dichte

6 Das Gitter wird bereits von der Firma NTT-AT (Japan) gefertigt. Aufgrund der langen Lieferzeiten kann es im Rahmen dieser Arbeit nicht mehr getestet werden. 

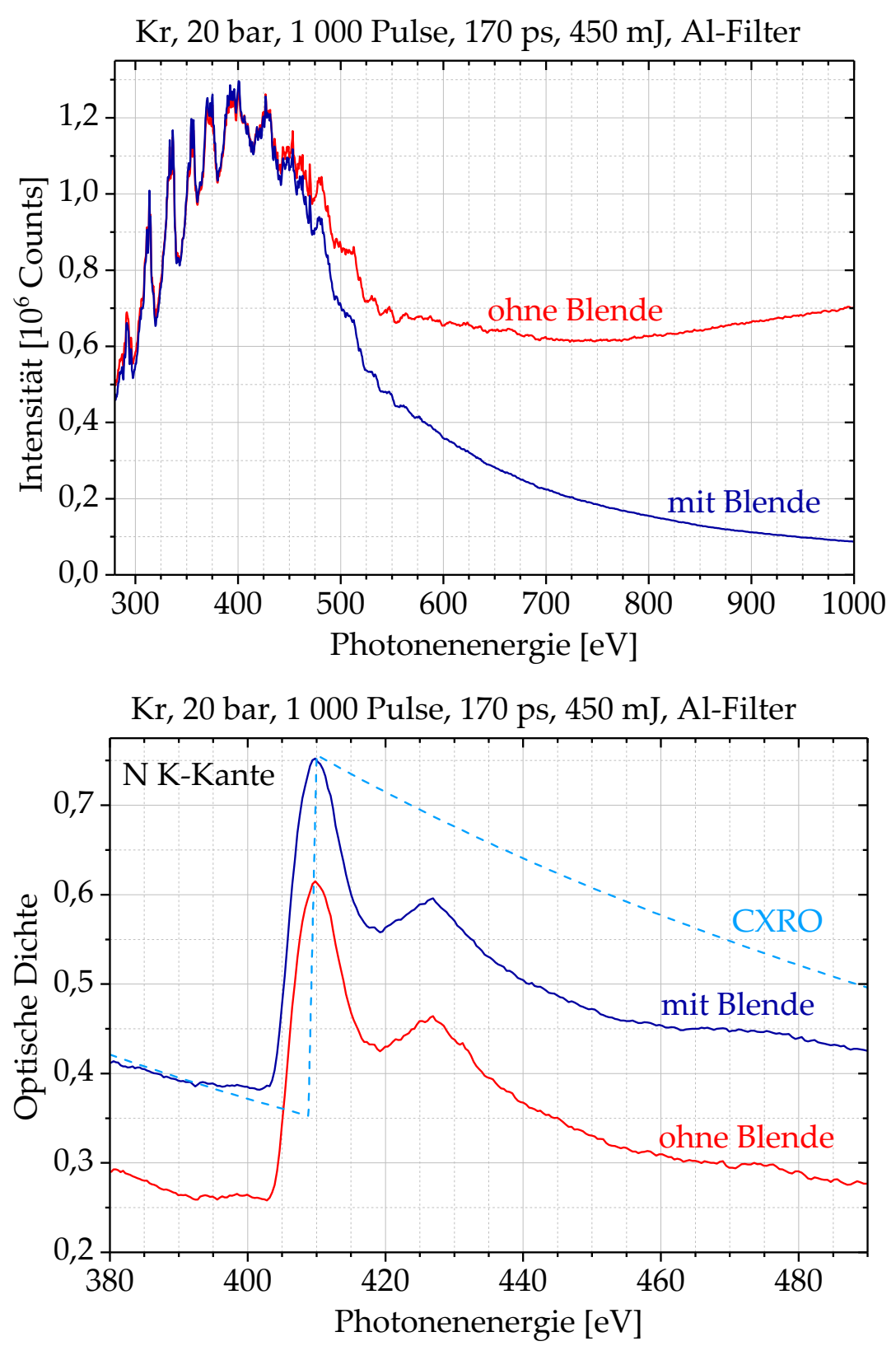

Abbildung 4.22: Emissionsspektren von Krypton (oben) und NEXAFS-Spektren von $\mathrm{Si}_{3} \mathrm{~N}_{4}$ (Dicke $100 \mathrm{~nm}$ ) an der Stickstoff-K-Kante (unten) mit und ohne Blende. Zudem ist die optische Dichte aus der CXRO-Datenbank [120] eingetragen. 
Kr, 20 bar, 1000 Pulse, 170 ps, 450 mJ, Al-Filter
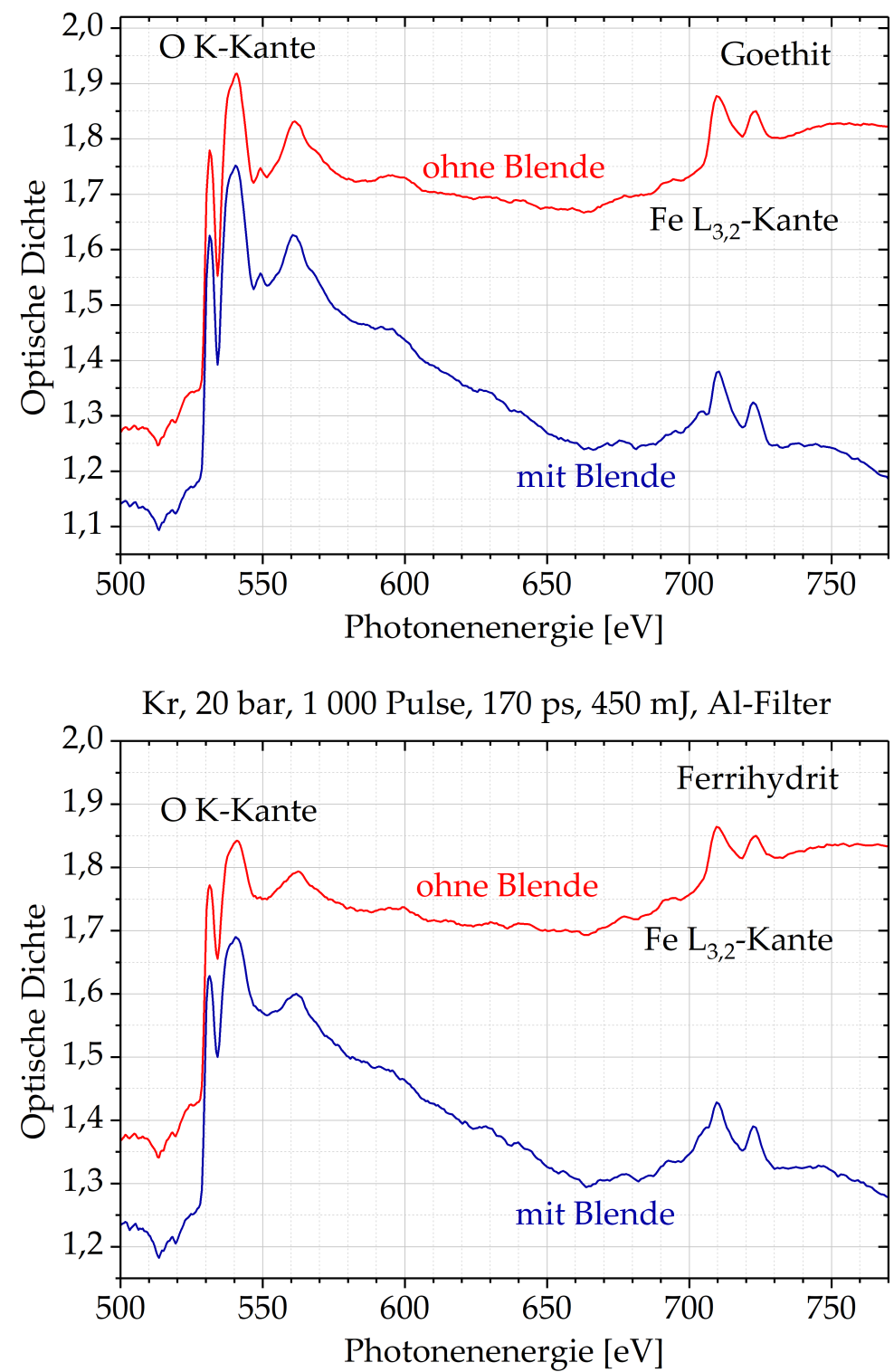

Abbildung 4.23: NEXAFS-Spektren von Goethit (oben) und Ferrihydrit (unten) an der Sauerstoff-K- und Eisen- $\mathrm{L}_{3,2}$-Kante mit und ohne Blende 
nimmt nach den Kanten ab und erreicht vor der Eisen-L,2-Kante fast den gleichen Wert wie vor der Sauerstoff-K-Kante. Im Spektralbereich weicher Röntgenstrahlung liegen die Absorptionskanten allerdings energetisch nah zusammen. Nach einer Kante erreicht die optische Dichte demnach nicht den ursprünglichen Wert, bevor die Absorption an der energetisch höher liegenden Kante bereits wieder ansteigt. Ohne Blende ist die Differenz zwischen den optischen Dichten vor den beiden Kanten jedoch um einen Faktor 4 bis 6 größer. Die 0. Beugungsordnung sollte daher stets ausgeblendet werden.

\section{$4 \cdot 7$}

PROBEN UNTER ATMOSPHÄRENDRUCK

Einige der in diesem Abschnitt beschriebenen Ergebnisse und gezeigten Abbildungen finden sich in ähnlicher Weise in der Publikation:

F.-C. Kühl, M. Müller, M. Schellhorn, K. Mann, S. Wieneke und K. Eusterhues, "Near-edge $\mathrm{x}$-ray absorption fine structure spectroscopy at atmospheric pressure with a table-top laser-induced soft x-ray source," Journal of Vacuum Science and Technology A 34(4), 041302 (2016).

Die Messungen mit der Heliumkammer (zum Aufbau siehe Abs. 4.4) erfolgten in Zusammenarbeit mit Frank-Christian Kühl. Das NEXAFSSpektrometer war hinsichtlich der Abstände zwischen Quelle und Eintrittsspalt noch nicht optimiert.

Vor der Messung von Proben in verschiedenen Umgebungen wurde zunächst der Einfluss der Heliumkammer auf das Emissionsspektrum des Kryptons untersucht (siehe Abb. 4.24). Die Spektren, die mit Helium bzw. Luft gefüllter Probenkammer gemessen wurden, weisen über den gesamten Energiebereich eine geringere Intensität auf als das unter Vakuumbedingungen aufgenommene Emissionsspektrum. Die Abnahme der Intensität stimmt dabei sehr gut mit der aus tabellierten Daten berechneten Transmission überein [120], die aufgrund der beiden $150 \mathrm{~nm}$ dicken $\mathrm{Si}_{3} \mathrm{~N}_{4}$-Membranen und der $4 \mathrm{~mm}$ langen Wegstrecke durch Helium bzw. Luft an Atmosphärendruck erwartet wird. Deutlich $\mathrm{zu}$ erkennen ist zudem die Absorptionskante des Stickstoffs der $\mathrm{Si}_{3} \mathrm{~N}_{4}$ Membranen bei $410 \mathrm{eV}$. Ist die Probenkammer mit Luft gefüllt, geht die Intensität infolge der zusätzlichen Absorption des Luftstickstoffs für Photonenenergien oberhalb der Stickstoff-K-Kante auf Null zurück. Um das Signal-Rausch-Verhältnis zu verbessern, werden die in Heliumatmosphäre bzw. Luft gemessenen NEXAFS-Spektren gegenüber den im Vakuum aufgenommenen Spektren daher über die doppelte Anzahl von Pulsen integriert. 


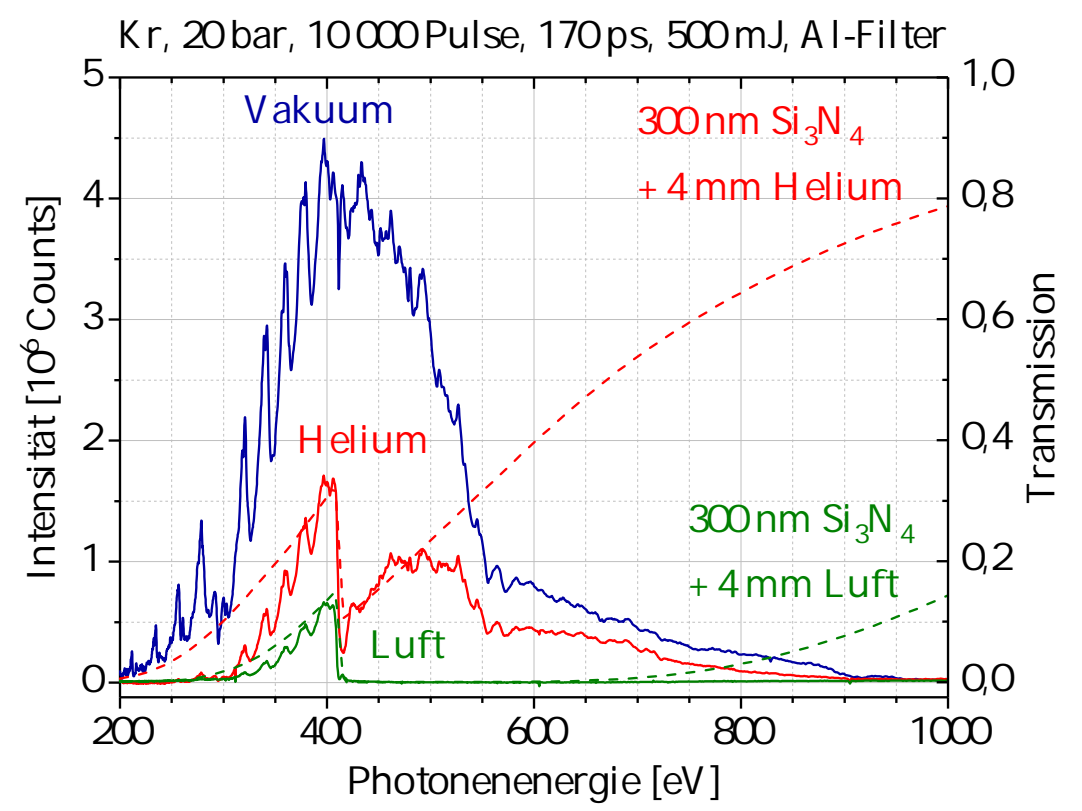

Abbildung 4.24: Emissionsspektren von Krypton, aufgenommen für verschiedene Bedingungen (Vakuum sowie Helium bzw. Luft bei Atmosphärendruck) in der Probenkammer. Zum Vergleich ist die Transmission von zwei jeweils $150 \mathrm{~nm}$ dicken $\mathrm{Si}_{3} \mathrm{~N}_{4}$-Membranen in Kombination mit $4 \mathrm{~mm}$ Helium bzw. Luft aufgetragen [120].

\section{KALIUMPERMANGANAT}

In Abbildung 4.25 sind die NEXAFS-Spektren einer Kaliumpermanganatprobe $\left(\mathrm{KMnO}_{4}\right)$ an der Sauerstoff-K- und Mangan- $\mathrm{L}_{3,2}$-Kante gezeigt, die im Vakuum sowie in Helium bzw. Luft bei Atmosphärendruck gemessen wurden. Wie zu erwarten, kann aufgrund der starken Absorption der weichen Röntgenstrahlung in diesem Energiebereich kein Signal in der mit Luft gefüllten Probenkammer gemessen werden. Im Vakuum bzw. mit Helium sind hingegen in den Spektren die Sauerstoff-K- und Mangan-L $\mathrm{L}_{3,2}$-Kante deutlich ausgeprägt. Die charakteristische Aufspaltung der Mangan-L-Kante in den $\mathrm{L}_{3}-\mathrm{Peak}$ bei 644,0 eV (Helium) bzw. $644,7 \mathrm{eV}$ (Vakuum) und den $\mathrm{L}_{2}$-Peak bei 655,1 eV (Helium und Vakuum) resultiert aus Elektronenübergängen aus dem 2p $p_{3 / 2^{-}}$bzw. $2 p_{1 / 2}$-Orbital in 3d-Orbitale. Der Unterschied für die Position des $\mathrm{L}_{3}$-Peaks bei Messung mit Helium bzw. im Vakuum $\left(\Delta_{\mathrm{L}_{3}} \mathrm{E}=0,7 \mathrm{eV}\right)$ ist geringer als die Energieauflösung, die für diesen Energiebereich etwa $\Delta \mathrm{E}=1,5 \mathrm{eV}$ beträgt. Insgesamt weichen die Peakpositionen um weniger als $0,1 \%$ von Messwerten ab, die von Zaharieva et al. [250] am Synchrotron bestimmt wurden $\left(\mathrm{L}_{3}: 644,41 \mathrm{eV}, \mathrm{L}_{2}: 655,18 \mathrm{eV}\right)$. 


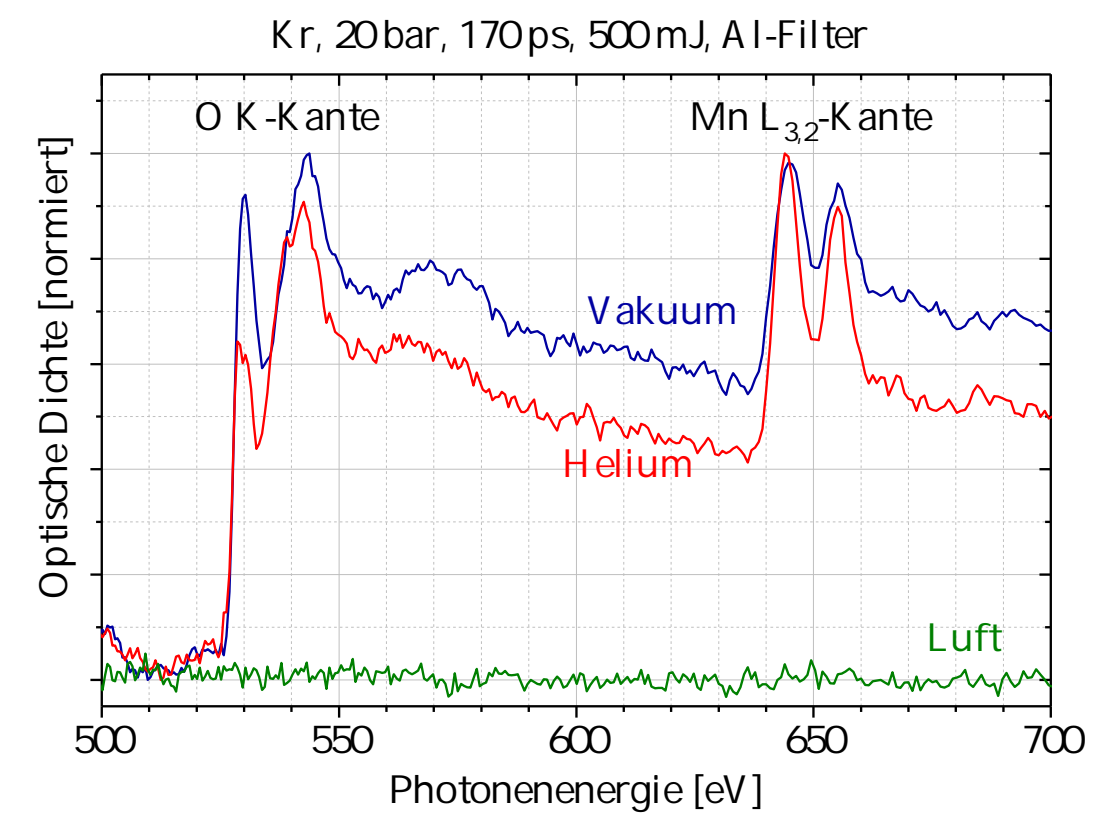

Abbildung 4.25: NEXAFS-Spektren von Kaliumpermanganat $\left(\mathrm{KMnO}_{4}\right)$ an der Sauerstoff-K- und Mangan-L, 3,2 -Kante, aufgenommen mit Probe im Vakuum (10 000 Pulse) sowie in Helium- bzw. Luftatmosphäre (20 000 Pulse)

\section{CALCIUMCHLORID}

Zur Charakterisierung der Probenkammer wurde neben Kaliumpermanganat auch Calciumchlorid $\left(\mathrm{CaCl}_{2} \cdot \mathrm{H}_{2} \mathrm{O}\right)$ gemessen. Die NEXAFS-Spektren an der Calcium-L- und Sauerstoff-K-Kante, die im Vakuum sowie in Helium bzw. Luft bei Atmosphärendruck gemessen wurden, sind in Abbildung 4.26 dargestellt. An der Calcium-L-Kante ist für alle drei Probenumgebungen die typische Aufspaltung in $\mathrm{L}_{3}$ - und $\mathrm{L}_{2}$-Kante sichtbar, die aus Elektronenübergängen aus dem $2 p_{3 / 2}$ - bzw. $2 p_{1 / 2}$-Orbital in $3 d-$ Orbitale folgt. Beide Peakpositionen bleiben dabei für die Messungen an Luft bzw. in Helium und im Vakuum gleich. Die Abweichung zu Synchrotrondaten beträgt weniger als 0,1\% [251].

Calciumchlorid ist stark hygroskopisch [252], nimmt daher Wasser aus der Umgebungsluft auf und bildet dabei einen Tetra- oder Hexahydratkomplex. Allerdings ist die Sauerstoff-K-Kante nur mit heliumgefüllter Probenkammer messbar. Im Vakuum verändert sich die Kristallstruktur des Calciumchlorids mit abnehmendem Umgebungsdruck vom Tetrabzw. Hexahydrat hin zu einer wasserfreien Rutil-Struktur [253], sodass kein Sauerstoffsignal detektiert werden kann. In Luft ist der Hydratkomplex zwar vorhanden, lässt sich aber aufgrund der starken Absorption der weichen Röntgenstrahlung oberhalb der Stickstoff-K-Kante bei $410 \mathrm{eV}$ (siehe Abb. 4.24) nicht messen. Um sensitive Proben wie Calciumchlorid korrekt zu analysieren, ist es demnach notwendig, die Probenkammer mit Helium zu befüllen. 
Kr, 20 bar, 170 ps, 500 mJ, Al-Filter

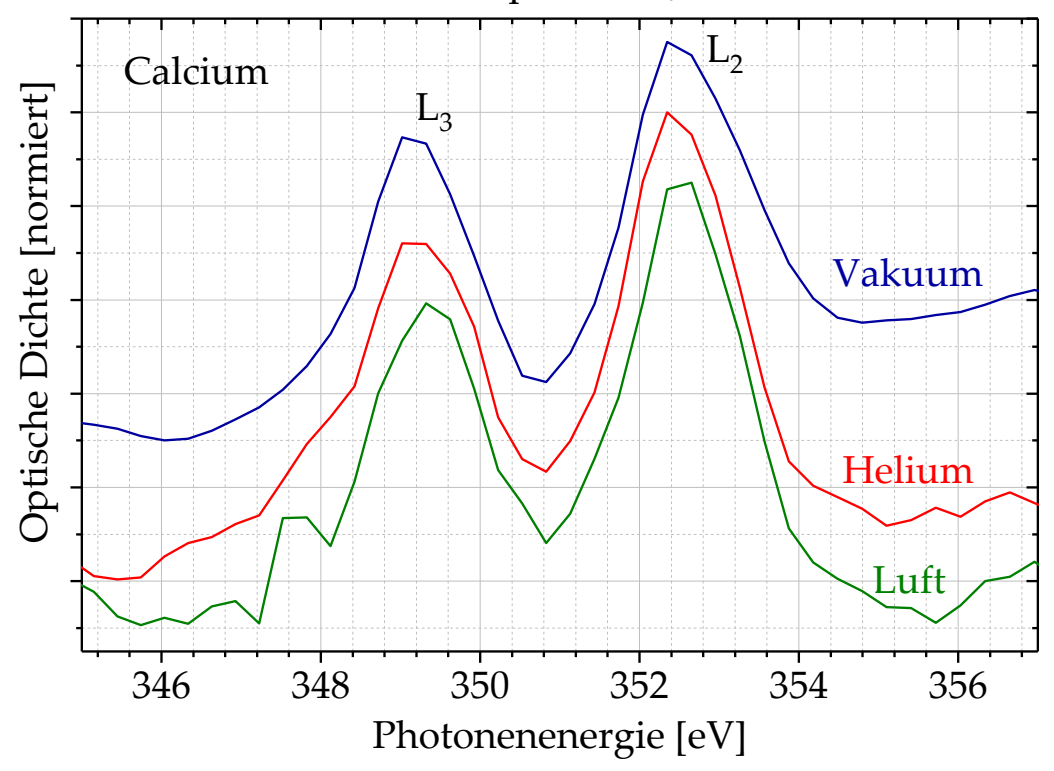

Kr, 20 bar, 170 ps, 500 mJ, Al-Filter

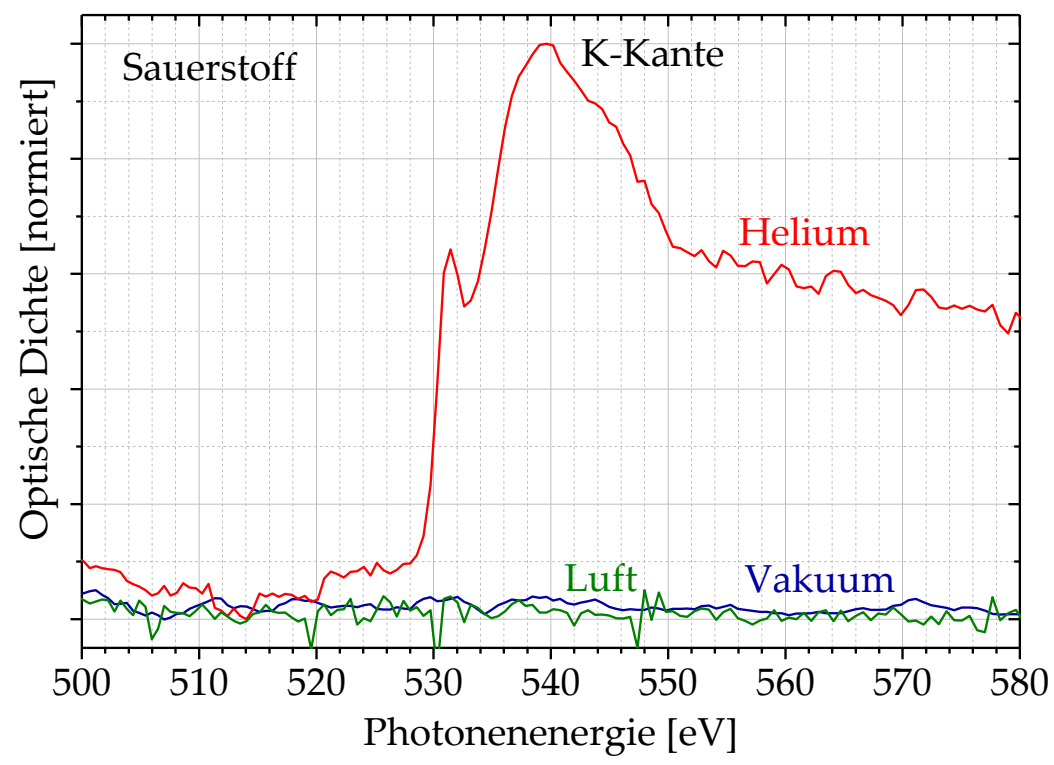

Abbildung 4.26: NEXAFS-Spektren von Calciumchlorid $\left(\mathrm{CaCl}_{2} \cdot \mathrm{H}_{2} \mathrm{O}\right)$ an der Calcium-L- (oben) und Sauerstoff-K-Kante (unten), aufgenommen für verschiedene Bedingungen (Vakuum, 10000 Pulse sowie Helium bzw. Luft bei Atmosphärendruck, 20000 Pulse) in der Probenkammer. Zur besseren Übersicht sind die Spektren entlang der $y$-Achse zueinander verschoben. 


\subsection{WEITERENTWICKLUNG DES NEXAFS-SPEKTROMETERS}

Durch die Verkürzung der Abstände zwischen Quelle, Probe und Eintrittsspalt des Spektrometers wurde das Signal-Rausch-Verhältnis und damit die Qualität der NEXAFS-Spektren insbesondere für Photonenenergien oberhalb von $500 \mathrm{eV}$ deutlich verbessert. Dadurch konnte die durchschnittliche Messzeit zur Aufnahme der Spektren um einen Faktor 5 bis 10 auf unter fünf Minuten verringert werden. Außerdem haben sich neue Anwendungsmöglichkeiten wie die Analyse der L-Kanten eisenhaltiger Proben ergeben. Allerdings reicht die bisherige spektrale Auflösung nicht aus, um die Aufspaltung der Peaks an der Eisen-L-Kante zu messen. Daher sollten in einem zukünftigen Spektrometer die bereits diskutierten Möglichkeiten zur Erhöhung des Auflösungsvermögens umgesetzt werden. Als Folge dessen nimmt das Signal-Rausch-Verhältnis jedoch ab, und zwar z.B. aufgrund der größeren Abstände zwischen Eintrittsspalt, Gitter und Detektor ab. Um diesen Intensitätsverlust auszugleichen, sollten bei der Weiterentwicklung des Spektrometers auch die in Abschnitt 2.3.4 und 2.3.5 beschriebenen Maßnahmen zur Brillanzerhöhung der Plasmaquelle berücksichtigt werden. Zudem können Referenz- und Probenspektrum gleichzeitig aufgenommen werden. Das verkürzt zum einen die Messzeit und kompensiert zum anderen Intensitätsschwankungen der Quelle. Hierfür würde allerdings ein Detektor mit 2048 statt nur 512 Pixeln benötigt.

Im Rahmen dieser Arbeit wurden NEXAFS-Spektren ausschließlich in Transmission aufgenommen. Für möglichst aussagekräftige Spektren dürfen die Proben dazu aber nicht wesentlich dicker sein als die Eindringtiefe der weichen Röntgenstrahlung an der Absorptionskante. Jedoch lassen nicht alle Proben zu, homogene Schichten mit Dicken im Bereich weniger Hundert Nanometer herzustellen. Solche Proben könnten allerdings in Reflexion statt in Transmission gemessen werden. Aufgrund der geringen Eindringtiefe $(<30 \mathrm{~nm})$ weicher Röntgenstrahlung unter streifendem Einfall ist diese Variante zudem besonders oberflächensensitiv. Der Vergleich zwischen Spektren einer Probe aufgenommen in Reflexion bzw. Transmission bietet so die Möglichkeit, den Einfluss von Oberflächeneffekten zu untersuchen. Die Messung von Spektren in Reflexion ist jedoch aufwendiger als in Transmission: Während in Transmission nur die Absorption $\beta$ der Probe von Bedeutung ist, kommt für Reflexionsmessungen die Brechung $\delta$ hinzu. Um beide unbekannte Größen bestimmen zu können, sind daher zwei Messungen z. B. unter geringfügig verschiedenen Einfallswinkeln notwendig. Diese Vorgehensweise ist jedoch sehr aufwendig, da für jeden Winkel das Gitter nachjustiert und die Skala des Detektors neu kalibriert werden müsste. Alternativ können Absorption und Brechung aus einer Messung mit einem iterativen Algorithmus berechnet werden, der von Picard-Lagnel et al. 
[254] vorgeschlagen wurde. Dieser nutzt aus, dass beide Größen über die Kramers-Kronig-Relation [255] miteinander verbunden sind. Ausgehend von der gemessenen, energieabhängigen Reflektivität $R_{0}$ für einen festen Einfallswinkel $\theta$ werden $\beta$ und $\delta$ aus vier Schritten ermittelt:

1. Berechnung von $\beta_{i}$ aus $\delta_{i-1}$

2. Berechnung von $\delta_{i}$ aus $\beta_{i}$ mit Kramers-Kronig-Relation

3. Berechnung von $R_{i}$ nach Fresnel-Gleichung (3.15)

4. Vergleich von $R_{0}$ mit $R_{i}$

Für die erste Iteration wird dabei ein zufällig gewähltes $\delta_{0}$ verwendet. Anschließend werden die vier Schritte so oft wiederholt, bis die Fehlerquadratsumme $\sum\left(R_{0}-R_{i}\right)^{2}$ minimal ist.

Mit Messungen in Reflexion ergeben sich zahlreiche neue Anwendungsmöglichkeiten des kompakten NEXAFS-Spektrometers insbesondere in der Oberflächenanalytik. Zuvor muss der Algorithmus allerdings weiterentwickelt werden, um u. a. negative Werte von $\delta$ an den Absorptionskanten zu berücksichtigen. Zudem muss untersucht werden, wie sich die Oberflächenrauheit der Proben auf die Spektren auswirkt.

Auch durch die Verwendung der Heliumkammer haben sich neue Anwendungen entwickelt, da nun Proben analysiert werden können, die nicht vakuumbeständig sind. Die Heliumkammer ermöglicht darüber hinaus den Einsatz von Fluidzellen, mit denen zeitaufgelöste Insitu-Messungen durchgeführt werden können. Dadurch eröffnen sich neue Perspektiven u. a. die Untersuchung chemischer Prozesse wie von Redoxreaktionen an Eisenoxiden. Bislang sind solche Messungen ausschließlich an Synchrotronquellen möglich (siehe z. B. Ref. [256-258]). 

Spektromikroskopie mit weicher Röntgenstrahlung bezeichnet ein analytisches Verfahren, mit dem sowohl die Form und Struktur als auch die chemische Zusammensetzung einer Probe untersucht werden können [259]. Dabei wird die hohe räumliche Auflösung der Röntgenmikroskopie mit der hohen Sensitivität der NEXAFS-Spektroskopie vereint. Dieses Verfahren ist daher ideal geeignet, um nano-strukturierte Proben wie Polymere, Proteine und Metalloxide zu messen, die aus mehreren Materialien zusammengesetzt sind. Gegenüber anderen spektromikroskopischen Methoden wie EELS (engl. electron energy loss spectroscopy) zeichnet sich die Röntgen-Spektromikroskopie durch höhere Eindringtiefen ins Material und eine geringere Strahlenbelastung der Probe aus [260]. Weitere Vorteile der NEXAFS-Spektroskopie wurden bereits in Abschnitt 4.I diskutiert.

\section{I SYNCHROTRONBASIERTE SPEKTROMIKROSKOPIE}

Um Proben spektromikroskopisch untersuchen zu können, bedarf es einer Röntgenquelle, deren Photonenenergie kontinuierlich verändert werden kann. Synchrotronstrahlquellen sind daher sehr gut für die Röntgen-Spektromikroskopie geeignet.

Wie in der Röntgenmikroskopie gibt es auch für die Spektromikroskopie verschiedene Abbildungstechniken: Die Probe lässt sich entweder im Ganzen abbilden oder wird Punkt für Punkt gerastert (siehe Abs. 3.1.4). Zusätzlich wird zwischen Methoden unterschieden, in denen die transmittierten Photonen (z. B. STXM - engl. scanning transmission $\mathrm{x}$ ray microscopy) oder die von der Probe emittierten Photoelektronen (z.B. PEEM - engl. photoemission electron microscopy) detektiert werden. Neben den klassischen Mikroskopieverfahren werden auch "linsenlose" Techniken wie die Ptychografie verwendet. Um ein NEXAFSSpektrum aufzunehmen, wird aber in allen Fällen die Energie der einfallenden Röntgenstrahlung im Bereich der Absorptionskante variiert und für jede Energie ein Mikroskopbild aufgenommen (siehe Abb. 5.1). Für jede der $n$ Photonenenergien absorbiert die Probe die Röntgenstrahlung unterschiedlich stark, sodass das Spektrum im gewünschten Bildfeld gemäß Lambert-Beer'schem Gesetz berechnet werden kann. Die Intensität I wird dabei an einer Stelle $(x, y)$ mit Probe, die Referenzintensität $I_{0}$ aus einem Bildausschnitt ohne Probe bestimmt. 

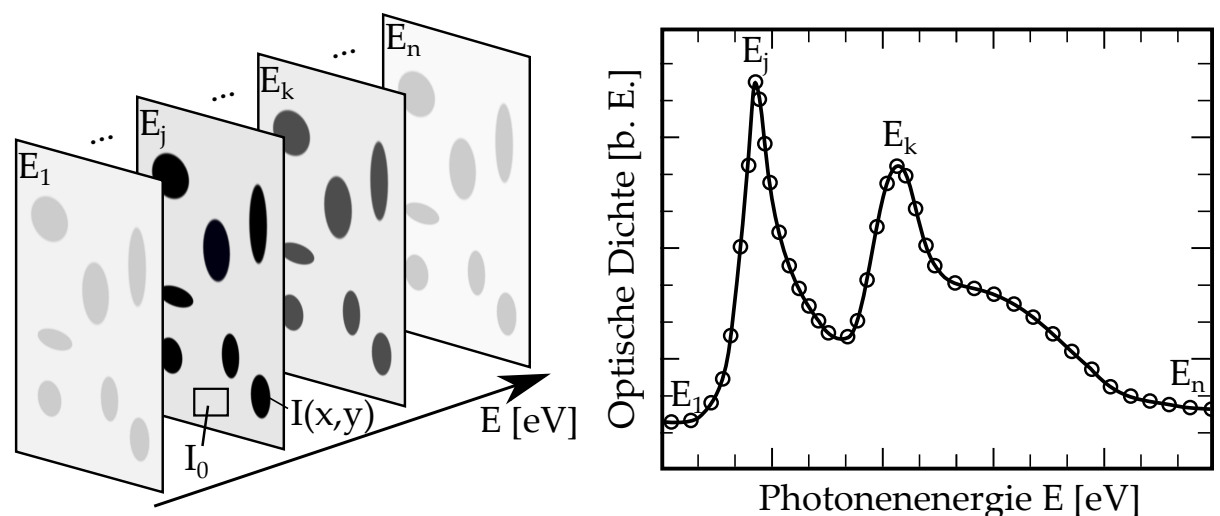

Abbildung 5.1: Schematische Darstellung zur Berechnung eines NEXAFSSpektrums aus Röntgenmikroskopbildern, aufgenommen bei verschiedenen Energien im Bereich der Absorptionskante

Die Energieauflösung synchrotronbasierter Spektromikroskope liegt zwischen $E / \Delta E=2000$ und $E / \Delta E=10000$ [261, 262], sodass bereits geringe Unterschiede in der chemischen Zusammensetzung der Probe detektiert werden können. Die hohe Sensitivität der NEXAFS-Spektroskopie ermöglicht zudem die Messung sehr niedriger Konzentrationen eines Elementes (z. B. 0,9 mg Kohlenstoff pro Gramm Probe [263]).

Die Spektromikroskopie an Synchrotronen ist nicht nur auf die zweidimensionale Abbildung von Objekten beschränkt. Es ist darüber hinaus möglich, Proben dreidimensional [264, 265], in wässriger Umgebung [266], zeitaufgelöst [18], in operando [267] oder in verschiedenen Kombinationen dieser Modi [268] abzubilden. Zusätzlich zur Absorption lässt sich auch die Phase als Funktion der Energie bestimmen [269]. Weitere Anwendungsbeispiele bieten u. a. die Referenzen [259, 261, 270].

\subsection{LABORAUFBAU}

Die Anforderungen an einen kompakten Aufbau für die Spektromikroskopie sind vielfältig: Zum einem muss die Quelle (quasi-)kontinuierlich über einen weiten Energiebereich emittieren, um möglichst viele Absorptionskanten unterschiedlicher Elemente zu erreichen. Als Quelle bietet sich daher ein laserinduziertes Kryptonplasma an, da dessen Emissionsspektrum entsprechend breitbandig ist. Zum anderen muss die Probe aber mit monochromatischer Strahlung belichtet werden, damit diese mit hoher Auflösung abgebildet werden kann. Die größte Schwierigkeit besteht nun darin, einen geeigneten Monochromator für die kompakte Laborquelle zu finden. Außerdem müssen für ein kompaktes Spektromikroskop zunächst die in Abschnitt 3.3.5 genannten Möglichkeiten zur Brillanzsteigerung der Quelle umgesetzt werden. Es wird trotzdem ein Kompromiss zwischen dem räumlichen Auflösungsvermögen und der chemischen Sensitivität nötig sein. 
Im folgenden Abschnitt werden zwei mögliche Ansätze zur Realisierung eines kompakten Spektromikroskops beschrieben. Sie basieren auf den bisherigen Aufbauten des Röntgenmikroskops (siehe Abs. 3.3.1) bzw. des NEXAFS-Spektrometers (siehe Abs. 4.4).

\subsubsection{ZONENPLATTENMONOCHROMATOR}

Um die vom Plasma emittierte Strahlung zu monochromatisieren, kann die Chromatizität einer Zonenplatte in Kombination mit einer energieselektiven Apertur ausgenutzt werden (siehe Abb. 5.2). Dabei gilt: Je
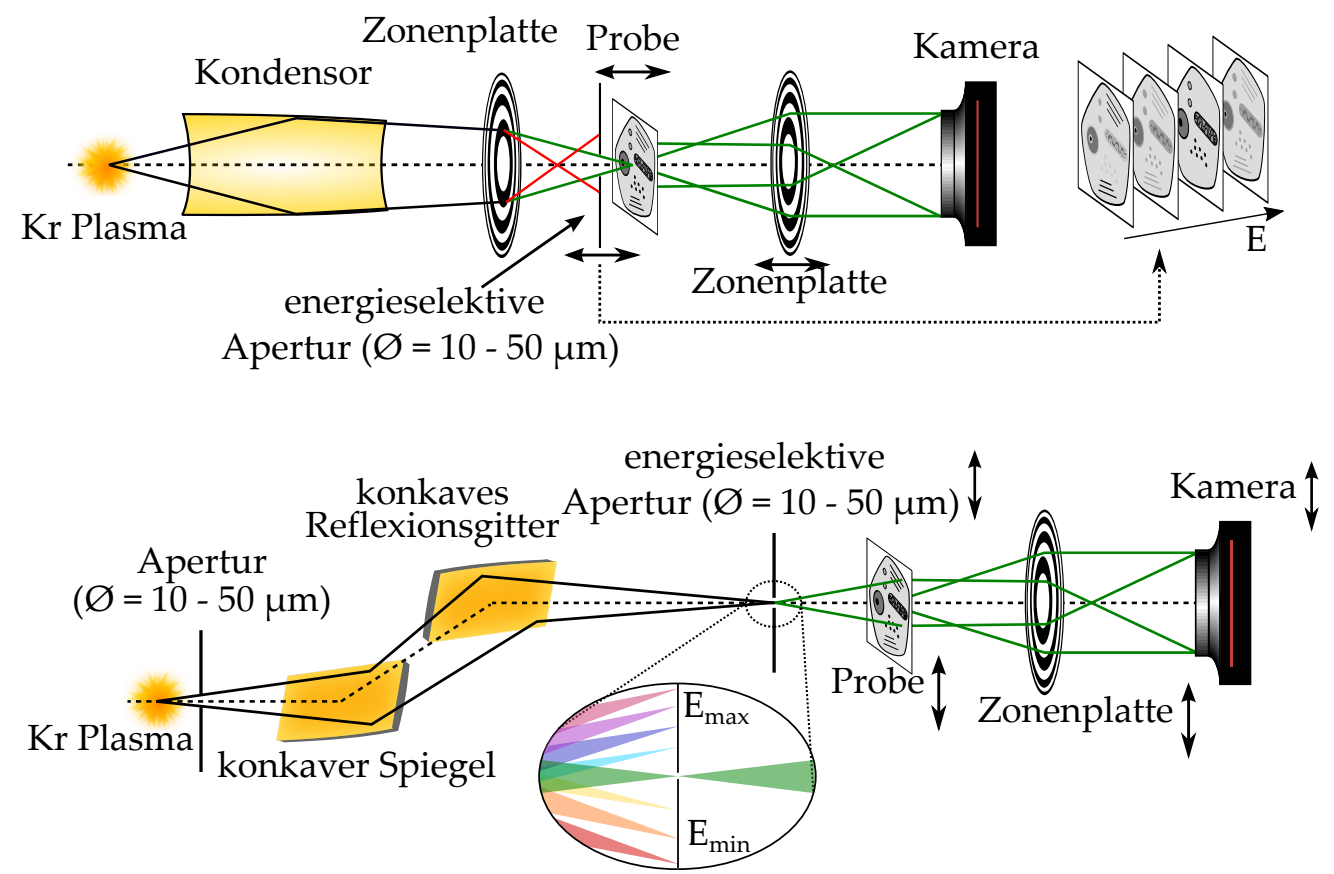

Abbildung 5.2: Skizzen möglicher Laboraufbauten für die Spektromikroskopie mit Zonenplatte (oben) bzw. konkavem Reflexionsgitter (unten) als Monochromator

kleiner die Blendenöffnung ist, desto besser ist die spektrale Auflösung, und die Intensität nimmt im selben Umfang ab. Aufgrund der geringen Effizienz der Zonenplatte (ca. $4 \%$ ) ist daher absehbar, dass die Belichtungszeiten entsprechend steigen werden. Ein großer Vorteil dieser Variante ist jedoch, dass sich die zusätzliche Zonenplatte und Apertur einfach in den bestehenden Mikroskopaufbau integrieren lassen.

\subsubsection{GITTERMONOCHROMATOR}

Alternativ zur Zonenplatte kann auf ein konkaves Reflexionsgitter zurückgegriffen werden (siehe Abb. 5.2). Es vereint Dispersion und Fokussierung entlang der $x$-Richtung in einem optischen Element. Allerdings 
ist ein zweiter Konkavspiegel nötig, um die Röntgenstrahlung auch in yRichtung zu fokussieren. Somit ergibt sich eine Anordnung vergleichbar einer Kirkpatrick-Baez-Optik. Im Fokus wird die gewünschte Photonenenergie wiederum durch eine Lochblende selektiert und die Abbildung des Objekts erfolgt wie im Röntgenmikroskop mit einer Zonenplatte.

\subsubsection{ZWEI-FARBMIKROSKOP}

Die bisher vorgestellten Ansätze zur Spektromikroskopie basieren auf einer quasi-kontinuierlichen Röntgenquelle und geeigneten Monochromatoren. Stattdessen können aber auch Stickstoff, Sauerstoff oder Neon als Targetgase für die Plasmaquelle genutzt werden. Ihre Emissionsspektren bestehen aus einzelnen, charakteristischen Linien (siehe Abb. 5.3), die im Bereich der L-Kanten von Eisen $(\lambda=1,75 \mathrm{~nm})$, Mangan $(\lambda=1,94 \mathrm{~nm})$ und Titan $(\lambda=2,70 \mathrm{~nm})$ sowie der K-Kante des Sauerstoffs $(\lambda=2,34 \mathrm{~nm})$ liegen. Um chemische Informationen zu erhalten, wird die Probe jeweils

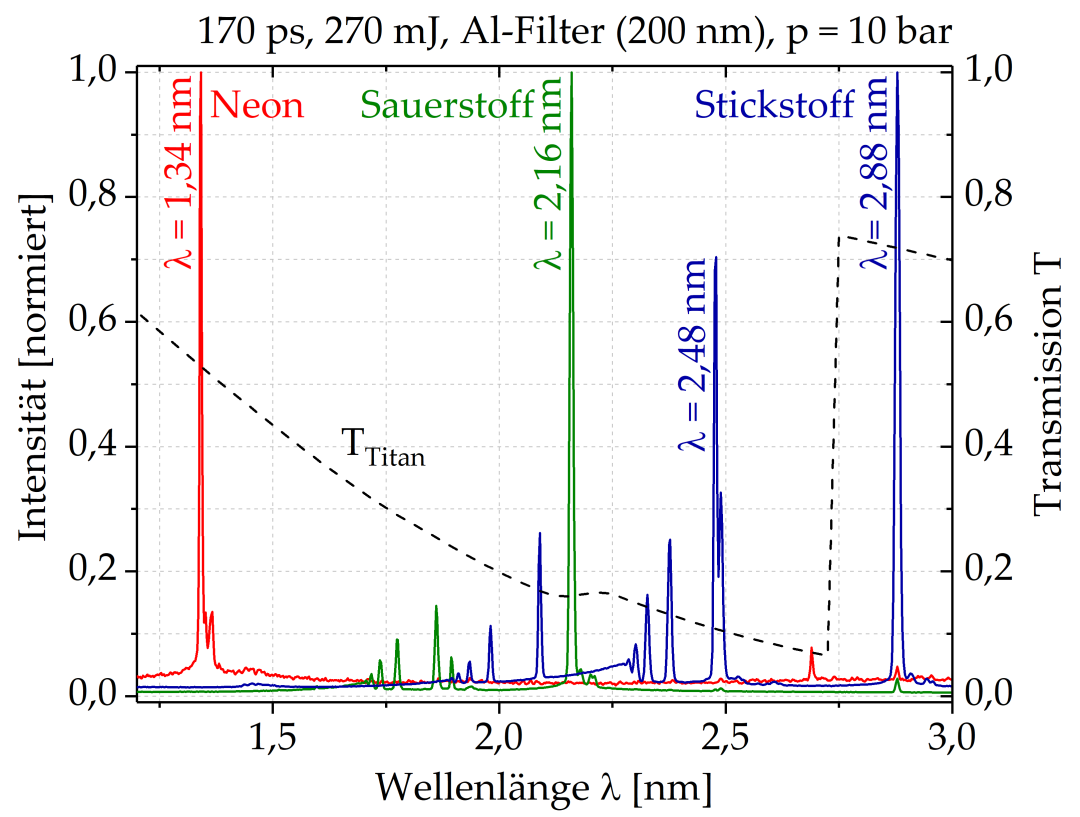

Abbildung 5.3: Normierte Emissionsspektren (100 Pulse) von Neon, Sauerstoff und Stickstoff und Transmission einer $215 \mathrm{~nm}$ dicken Titanfolie [120] im Spektralbereich von $\lambda=1,2$ bis $3,0 \mathrm{~nm}$

bei einer Energie ober- und unterhalb der Absorptionskante abgebildet. Dadurch kann zwar nicht die Feinstruktur der Kante, wohl aber die räumliche Verteilung des absorbierenden Elements gemessen werden. $\mathrm{Zu}$ beachten ist, dass die einzelnen Linien unterschiedlich intensiv sind. So ist $\mathrm{z}$. B. die Neonlinie bei $\lambda=1,34 \mathrm{~nm}$ circa um den Faktor 10 dunkler als die Stickstofflinie bei $\lambda=2,88 \mathrm{~nm}$. Bei der Abbildung eines Objekts sollte die Belichtungszeit dementsprechend angepasst werden. Zudem ist der Abstand der Neonlinie zur Eisen-L-Kante und Sauerstofflinie 
recht hoch, sodass die Transmissionsunterschiede eisenhaltiger Proben vor und nach der Kante geringer sind.

Im Gegensatz zum bisherigen Betrieb des Röntgenmikroskops wird keine Titan-, sondern eine Aluminiumfolie zum Blocken des sichtbaren Lichts und gestreuter Laserstrahlung verwendet. Damit wird sichergestellt, dass auch Röntgenstrahlung unterhalb der Titan-L-Kante bei $\lambda=2,7 \mathrm{~nm}$ genutzt werden kann. Mit Stickstoff als Targetgas wird das Objekt allerdings nicht mehr mit monochromatischem Licht, sondern mit 2,48 nm- und 2,88 nm-Strahlung beleuchtet ${ }^{1}$. Die Abbildung eines Objekts ist daher durch ein unscharfes "Zwillingsbild“ der jeweils anderen Wellenlänge überlagert. Wie stark dieser Einfluss ist, wurde zunächst am Beispiel einer $10 \mu \mathrm{m}$-Lochblende untersucht (siehe Abb. 5.4). Um zwischen den Wellenlängen zu wechseln, wurde die Zonenplatte
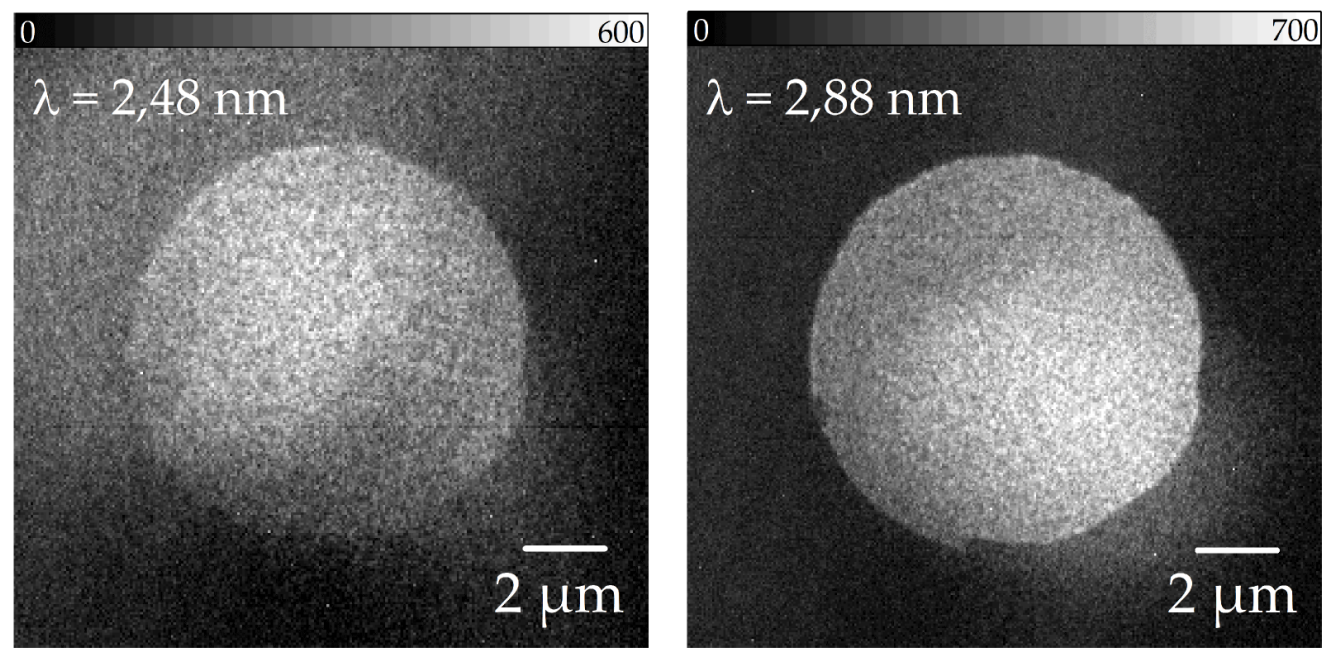

Abbildung 5.4: Mikroskopaufnahme $\left(M=210\right.$ bzw. $\left.M=245, t=20 \mathrm{~s}, \mathrm{ZP}_{3}\right)$ einer $10 \mu \mathrm{m}$-Lochblende, aufgenommen bei der Wellenlänge $\lambda=2,48 \mathrm{~nm}$ (links) bzw. $\lambda=2,88 \mathrm{~nm}$ (rechts)

um $170 \mu \mathrm{m}$ entlang der optischen Achse verfahren. Da lediglich über 100 Pulse integriert wurde, ist der Kontrast in beiden Aufnahmen sehr gering $\left(C_{M} \approx 0,05\right)$. Insbesondere in der $2,88 \mathrm{~nm}$-Aufnahme ist zudem das unscharfe Abbild der Lochblende zu erkennen, das durch die 2,48 nmStrahlung verursacht wird. Dennoch ist die Apertur für beide Wellenlängen eindeutig zu erkennen. Da der Abstand zwischen Probe und Kamera nicht verändert wurde, ist der Abbildungsmaßstab für die beiden Aufnahmen unterschiedlich.

Ausgehend von diesem Vorversuch wurde eine $100 \mathrm{~nm}$ dünne $\mathrm{Si}_{3} \mathrm{~N}_{4}$ Membran abgebildet, die teilweise mit einer Titanfolie (Dicke $215 \mathrm{~nm}$ ) bedeckt ist (siehe Abb. 5.5). Unterhalb der Absorptionskante des Titans $(\lambda=2,7 \mathrm{~nm})$ ist im Mikroskopbild nur die unbeschichtete Seite der

I Alle anderen Emissionslinien sind nicht intensiv genug, um die Abbildung negativ zu beeinflussen. 

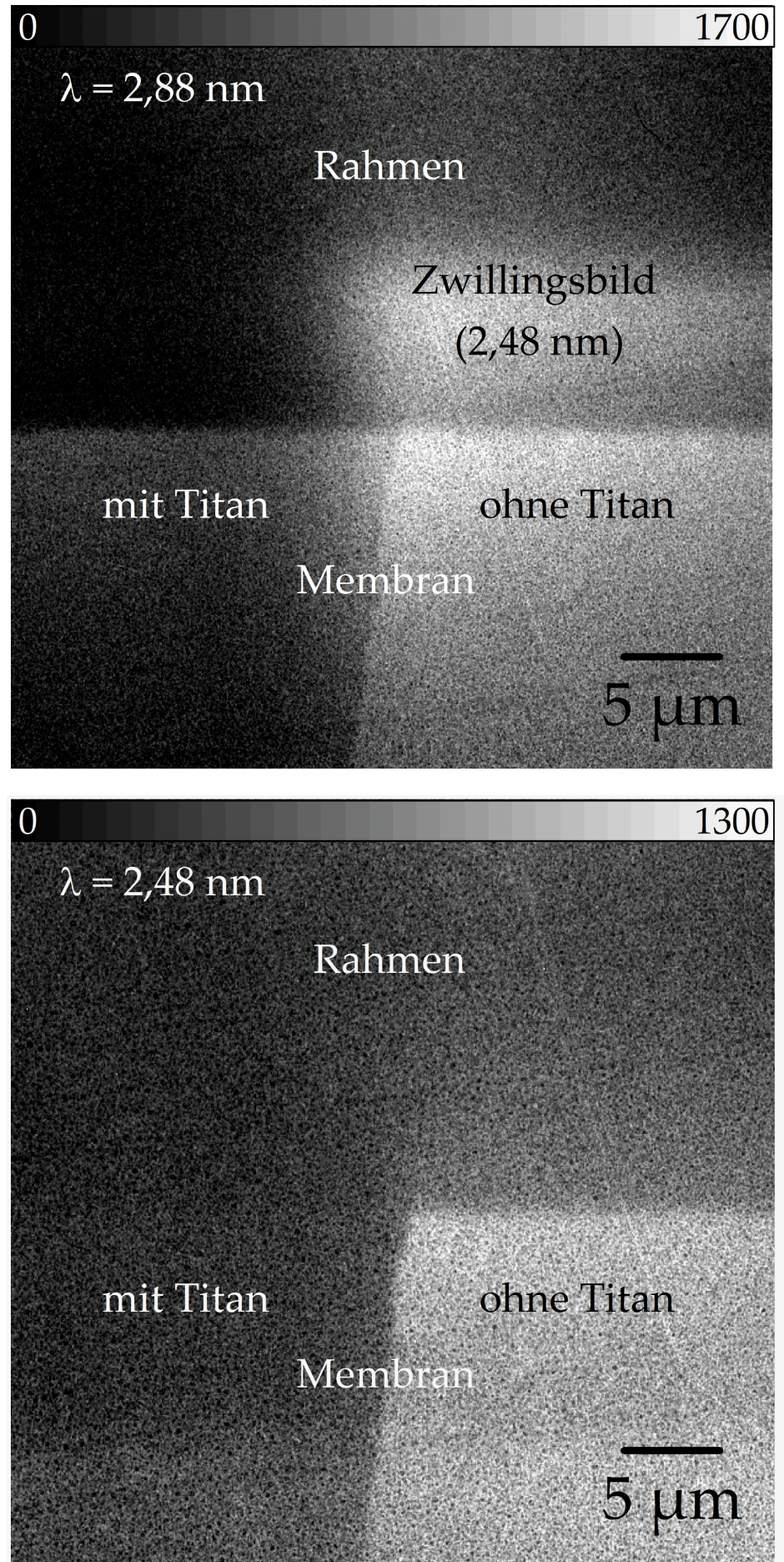

Abbildung 5.5: Mikroskopaufnahme $\left(M=210\right.$ bzw. $\left.M=245, t=30 \mathrm{~min}, \mathrm{ZP}_{3}\right)$ einer zum Teil mit Titanfolie (Dicke $215 \mathrm{~nm}$ ) bedeckten $\mathrm{Si}_{3} \mathrm{~N}_{4}$-Membran, aufgenommen bei der Wellenlänge $\lambda=2,48 \mathrm{~nm}$ (oben) bzw. $\lambda=2,88 \mathrm{~nm}$ (unten) 
Membran sichtbar, da die 2,48 nm-Strahlung von der Titanfolie nahezu vollständig absorbiert wird. In der 2,88 nm-Aufnahme oberhalb der Kante sind hingegen beide Bereiche und ihr Übergang zu sehen. Wäre die Membran mit anderen Metallen bedeckt, könnte kein signifikanter Unterschied festgestellt werden, da die Strahlung beider Wellenlängen im selben Maß transmittiert oder absorbiert worden wäre. 

Die Nachfrage nach Messzeit an synchrotronbasierten Röntgenmikroskopen und -spektrometern steigt stetig. Weltweit stehen aber nur wenige geeignete Synchrotronstrahlquellen zur Verfügung. Es besteht daher ein hoher Bedarf an kompakten Quellen zur Erzeugung weicher Röntgenstrahlung. Laserinduzierte Plasmen mit Gastarget sind dafür sehr gut geeignet. Die Brillanz dieser Quellen ist aufgrund der geringen Dichte des Gases zwar niedriger als bei Plasmaquellen mit flüssigen oder festen Targets, sie zeichnen sich aber durch eine hohe Langzeitstabilität, eine extrem kompakte Bauweise und einfache Handhabung aus. Diese drei Eigenschaften sind eine entscheidene Voraussetzung für eine weite Verbreitung dieser Quellen sowie der darauf basierenden Laborgeräte für die Röntgenmikroskopie und Absorptionsspektroskopie. Aus diesem Kontext haben sich drei Hauptziele ergeben:

1. Die Brillanz der Plasmaquelle mit Gastarget muss erhöht werden.

2. Mit dieser Quelle soll ein Laborröntgenmikroskop aufgebaut und

3. ein kompaktes NEXAFS-Spektrometer weiterentwickelt werden.

Die wichtigsten Ergebnisse dieser drei Arbeitsschwerpunkte werden im Folgenden zusammengefasst.

\section{QUELLENTWICKLUNG}

Die Brillanz des laserinduzierten Plasmas hängt maßgeblich vom Anregungslaser, der Gasdichte im Laserfokus und der Quellgröße ab. Im ersten Schritt wurde daher zwecks Brillanzerhöhung der Quelle der Einfluss der Laserpulslänge untersucht. Wird statt eines Nano- ein Pikosekundenlaser mit gleicher Pulsenergie verwendet, nimmt die Intensität der Plasmen um etwa eine Größenordnung zu. Gleichzeitig verringert sich die Plasmagröße um fast den Faktor 2. Die Emissionsspektren verschieben sich zudem zu höheren Photonenenergien, da die Plasmen aufgrund der höheren Leistungsdichte des Pikosekundenlasers insgesamt stärker aufgeheizt und höher angeregt werden. Diese Beobachtung konnte durch die mit dem Programm PrismSPECT berechneten Emissionsspektren von Argon bestätigt werden: Für den Pikosekundenlaser ergeben sich eine etwa $30 \%$ höhere Elektronentemperatur und eine 3 fach höhere Elektronendichte. Zukünftig könnte im Rahmen der Kooperation mit dem HiLASE Centre (Prag) untersucht werden, inwiefern sich noch kürzere Pulslängen $<5$ ps auf die Plasmaemission auswirken. 
Die Gasdichte im Laserfokus wurde durch eine erstmals eingesetzte Hochdruckdüse gesteigert, mit der sich der Gasdruck von 20 auf 100 bar erhöhen ließ. Damit konnte unter Verwendung des Pikosekundenlasers ein 5 fach intensiveres Stickstoffplasma induziert werden. Allerdings wird dabei die Wolframdüse beschädigt, da der Laserstrahl vom Plasma aufgeweitet und Material vom Düsenplättchen ablatiert wird. Bei diesem Gasdruck ist daher kein langzeitstabiler Betrieb der Quelle möglich. Um dies zu verhindern, sollen zukünftig andere Düsenmaterialien (z. B. Keramiken) eingesetzt werden. Zusätzlich lässt sich der Gasstrahl im Laserfokus weiter verdichten, wenn z. B. zwei Düsen senkrecht gegenüber angeordnet werden. In ersten Vorarbeiten wurde mit diesem Aufbau bereits ein helleres und kleineres Plasma erzeugt.

Weiterhin ist die Brillanz der Plasmaquelle um einen weiteren Faktor 6 höher, wenn die emittierte Röntgenstrahlung unter einem Winkel von $30^{\circ}$ statt $90^{\circ}$ zum einfallenden Laserstrahl genutzt wird. Ursache ist vor allem die auf etwa ein Viertel verringerte Plasmaausdehnung, aber auch die geringere Reabsorption der Strahlung im Gasstrahl.

Insgesamt lässt sich die Brillanz der Quelle um etwas mehr als zwei Größenordnungen steigern, wenn die bisher einzeln untersuchten Maßnahmen zukünftig kombiniert werden.

\section{HOCHAUFLÖSENDE BILDGEBUNG}

Auf Basis des laserinduzierten Plasmas mit gasförmigem Target wurde erstmals ein Mikroskop für weiche Röntgenstrahlung bei einer Wellenlänge von $\lambda=2,88 \mathrm{~nm}$ aufgebaut, mit dem ein besseres Auflösungsvermögen als mit konventionellen Lichtmikroskopen erreicht wird. Derzeit können Strukturen mit einer Größe von etwa $50 \mathrm{~nm}$ abgebildet werden. Die Belichtungszeit zur Aufnahme von Objekten liegt dabei zwischen $30 \mathrm{~min}$ bei einer Hellfeldabbildung und $120 \mathrm{~min}$ im Dunkelfeldkontrast. Bei der Untersuchung biologischer und geologischer Objekte stellte sich jedoch heraus, dass lediglich deren äußere Form sichtbar war, aber keine innere Struktur aufgenommen werden konnte. Ursache ist die noch immer zu geringe Brillanz der Quelle und damit das niedrige SignalRausch-Verhältnis der Mikroskopbilder. Allerdings könnten in einem zukünftigen Aufbau die beschriebenen Maßnahmen zur Erhöhung der Brillanz umgesetzt werden, um auch das Innere der Proben abbilden zu können. Zudem verspricht die Kombination von Röntgen- und STEDMikroskopie aufgrund der unterschiedlichen Kontrastverfahren der beiden Abbildungsmethoden mehr Informationen über die untersuchten Objekte zu gewinnen.

Zusätzlich zur „,klassischen“ Röntgenmikroskopie wurde als Vorarbeit zur „linsenlosen“ Bildgebung (CDI) die Kohärenz des laserinduzierten Plasmas untersucht. Dazu wurde die Kohärenzlänge der Quelle zum einen mit dem Van-Cittert-Zernike-Theorem direkt aus der Intensitäts- 
verteilung des Plasmas mit Quellapertur und zum anderen anhand des Beugungsbildes einer Lochblende berechnet. Aus beiden Methoden ergab sich in sehr guter Übereinstimmung in $1 \mathrm{~m}$ Entfernung zur Quelle eine Kohärenzlänge von ungefähr $13 \mu \mathrm{m}$.

\section{ABSORPTIONSSPEKTROSKOPIE}

Weiche Röntgenstrahlung eignet sich nicht nur für hochauflösende Abbildungsmethoden, sondern auch für die NEXAFS-Spektroskopie. Aus der Feinstruktur der Absorptionskante lassen sich zahlreiche Informationen wie die beteiligten Bindungstypen herleiten. Aus den Spektren ergibt sich somit quasi der "chemische Fingerabdruck" einer Probe. Im Rahmen dieser Arbeit konnte ein bereits vorhandenes NEXAFS-Spektrometer signifikant weiterentwickelt werden. Insbesondere durch kürzere Abstände zwischen Quelle, Probe und Eintrittsspalt des Spektrometers wurde das Signal-Rausch-Verhältnis der gemessenen Spektren erhöht. Damit sind erstmals NEXAFS-Messungen für Photonenenergien über $500 \mathrm{eV}$ bei Messzeiten von weniger als 5 min möglich. Das Auflösungsvermögen des Spektrometers liegt bei einer Energie von $430 \mathrm{eV} \mathrm{im}$ Bereich von $E / \Delta E=375$ bis 500 .

Mit dem optimierten Spektrometer wurde zunächst an Agar-100 der Einfluss der Dicke der Messprobe auf die Spektrenqualität untersucht. Übertrifft die Probendicke die Eindringtiefe der weichen Röntgenstrahlung, kommt es zur „unvollständigen Absorption“ und die NEXAFSSpektren können nicht mehr nach dem Lambert-Beer'schen Gesetz berechnet werden. Um korrekte Spektren in Transmission messen zu können, müssen die Proben daher entsprechend dünn sein. Weil aber nicht für alle Materialien homogene Schichten mit Dicken im Bereich weniger Hundert Nanometer präpariert werden können, sollen zukünftig auch Messungen in Reflexion erprobt werden.

Außerdem wurden NEXAFS-Spektren verschiedener Eisenoxide aufgenommen. Selbst Proben mit einer Schichtdicke von lediglich $25 \mathrm{~nm}$ konnten an der Sauerstoff-K- und Eisen-L-Kante vermessen werden. Die hohe chemische Sensitivität der NEXAFS-Spektroskopie wird demnach auch mit dem Laborspektrometer erreicht. Die NEXAFS-Spektren zeigen zudem eine sehr gute Übereinstimmung mit entsprechenden Synchrotrondaten. Die Auflösung ist verglichen mit synchrotronbasierten Spektrometern jedoch zu gering, um alle Peaks der Eisen-L-Kante zu sehen. Das Auflösungsvermögen des Spektrometers soll daher mit einem verbesserten Gitter um den Faktor 2 bis 3 gesteigert werden.

Bisher konnten die Proben im Laborspektrometer ausschließlich im Vakuum vermessen werden. Diese Einschränkung wurde durch den Bau einer kompakten Probenkammer überwunden, in der sich Proben an Luft oder in einer Heliumatmosphäre untersuchen lassen. Am Beispiel des stark hygroskopischen Calciumchlorids wurde daraufhin gezeigt, 
dass die Heliumumgebung zwingend erforderlich ist, um das aus der Hydrathülle stammende Sauerstoffsignal der Probe zu detektieren.

Darüber hinaus wurden zwei mögliche Ansätze für die Spektromikroskopie mit Laborgeräten und ein Zwei-Farbmikroskop in ersten Testversuchen evaluiert. Mit Letzterem konnte bereits gezeigt werden, dass ein Testobjekt bei den beiden Wellenlängen $\lambda=2,48 \mathrm{~nm}$ und $\lambda=2,88 \mathrm{~nm}$ unter- und oberhalb der Titan-L-Kante abgebildet werden kann.

In dieser Arbeit wurde somit anhand ausgewählter Beispiele gezeigt, dass Röntgenmikroskopie und Absorptionsspektroskopie mit kompakten Laborgeräten auf Basis eines laserinduzierten Plasmas mit gasförmigem Target möglich sind. Zudem wurden verschiedene Maßnahmen diskutiert, mit denen Quelle, Röntgenmikroskop und NEXAFS-Spektrometer hinsichtlich ihrer Brillanz und ihrer Anwendungsmöglichkeiten weiter verbessert werden können. Dabei bleibt die kompakte Bauweise, die hohe Stabilität und die einfache Handhabung der Laborgeräte erhalten. 


\section{A.I INSTRUMENTIERUNG}

Tabelle A.1: Übersicht der wichtigsten im Rahmen dieser Arbeit genutzten Messgeräte, ihrer Anwendungen und technischen Kenngrößen

\begin{tabular}{|c|c|c|}
\hline & Anwendung / Messparameter & technische Kenngrößen \\
\hline 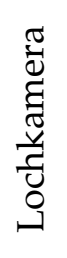 & $\begin{array}{l}\text { Plasmacharakterisierung } \\
\text { Intensität, Größe, Lage- } \\
\text { stabilität, typischerweise Auf- } \\
\text { nahme von } 1 \text { bis } 100 \text { Pulsen }\end{array}$ & $\begin{array}{l}\text { CCD-Chip SonyICX285, Pixelgröße } \\
6,45 \mu \mathrm{m}, 1280 \text { x } 1024 \text { Pixel, Phosphor } \\
\text { beschichtet (ProxiVision, P43, Dicke } \\
5 \mu \mathrm{m} \text { ), } 50 \mu \mathrm{m} \text {-Blende, } 200 \mathrm{~nm} \text {-Titan- } \\
\text { filter, Röntgenstrahlung } \lambda<20 \mathrm{~nm}\end{array}$ \\
\hline 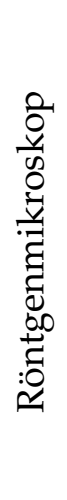 & $\begin{array}{l}\text { hochauflösende Abbildung, } \\
\text { Hell- und Dunkelfeldkontrast, } \\
\text { monochromatische Strahlung } \\
\lambda=2,88 \mathrm{~nm} \text {, Vergrößerung } 175 \\
\text { bis } 500 \text {, Auflösung } 50 \mathrm{~nm} \text {, typ. } \\
\text { Messzeit } 5 \text { bis } 120 \mathrm{~min}\end{array}$ & $\begin{array}{l}\text { Nanosekundenlaser Quantel (Para- } \\
\text { meter siehe Tab. 2.1), Parameter } \\
\text { Ellipsoid siehe Abb. 3.10, Fokus- } \\
\text { durchmesser } \approx 200 \mu \mathrm{m} \text {, Parameter } \\
\text { Zonenplatten siehe Tab. 3.2, rück- } \\
\text { seitig belichteter, gedünnter CCD- } \\
\text { Chip (Roper Scientific, Pixelgröße } \\
13 \mu \mathrm{m}, 1024 \times 1024 \text { Pixel bzw. Andor, } \\
\text { Pixelgröße } 13,5 \mu \mathrm{m}, 2048 \times 2048 \\
\text { Pixel), QE } \approx 80 \%(\lambda=2,88 \mathrm{~nm})\end{array}$ \\
\hline 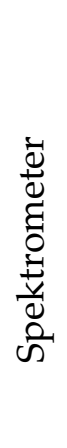 & $\begin{array}{l}\text { spektrale Charakterisierung } \\
\text { Emissionsspektren, NEXAFS- } \\
\text { Spektroskopie Messung } \\
\text { optische Dichte, Energiebereich } \\
250 \text { bis } 1250 \mathrm{eV}, \text { Auflösungs- } \\
\text { vermögen } \mathrm{E} / \Delta \mathrm{E} \approx 375 \text { bis } 500 \\
(\mathrm{E}=430 \mathrm{eV}), \text { typ. Messzeit } \\
\text { Einzelpuls bis } 5 \text { min, Helium- } \\
\text { kammer bis } 60 \mathrm{~min}\end{array}$ & $\begin{array}{l}\text { Nano- und Pikosekundenlaser } \\
\text { Innolas bzw. Ekspla (Parameter siehe } \\
\text { Tab. 2.I), Spaltbreite } 10 \text { bis } 400 \mu \mathrm{m}, \\
\text { typ. } 100 \mu \mathrm{m} \text {, Parameter Reflexions- } \\
\text { gitter siehe Abb. 4.4, rückseitig } \\
\text { belichteter, gedünnter CCD-Chip } \\
\text { (Roper Scientific, Pixelgröße } 13 \mu \mathrm{m}, \\
2048 \times 512 \text { Pixel, QE } \approx 60 \text { bis } 95 \% \\
(\mathrm{E}=250 \text { bis } 1250 \mathrm{eV})\end{array}$ \\
\hline
\end{tabular}




\section{A.2 PRISMSPECT SIMULATIONSPARAMETER}

- Element: Argon $(Z=18)$

- Model type: Detailed configuration accounting (DCA)

- Atomic Model: Emission Visible/UV/EUV Spectroscopy

- Atomic Rate Equations Solution: Steady-state

- Plasma Variables: Spherical geometry, Maxwellian Electron Distribution, Ion Density, Size $\Delta \mathrm{L}=500 \mu \mathrm{m}$

- External Radiation Source: None

- Independent Variables: Plasma Temperature, Ion Density

- Atomic Level Populations Model: LTE

\section{A.3 FITPARAMETER}

Tabelle A.2: Fitparameter der Lorentzkurvenkurven $\left(y=y_{0}+\frac{2 A}{\pi} \frac{w}{4\left(x-x_{c}\right)^{2}+w^{2}}\right)$, die an die Intensitätsprofile im Fokus des elliptischen Kondensorspiegels (siehe Abb. 3.12) angepasst wurden.

\begin{tabular}{cccc}
\hline & \multirow{2}{*}{ Parameter } & \multicolumn{2}{c}{ Wert } \\
& & horizontal & vertikal \\
\hline \multirow{2}{*}{ Konfiguration A } & $x_{c}[\mathrm{~mm}]$ & $4,9525(7)$ & $3,6038(9)$ \\
& $A[\mathrm{~mm}]$ & $0,640(3)$ & $0,619(4)$ \\
& $w[\mathrm{~mm}]$ & $0,4073(21)$ & $0,3944(26)$ \\
& $y_{0}[-]$ & $0,00580(6)$ & $-0,0062(26)$ \\
\hline Konfiguration B & $x_{c}[\mathrm{~mm}]$ & $3,985(3)$ & $2,976(2)$ \\
& $A[\mathrm{~mm}]$ & $0,232(8)$ & $0,246(6)$ \\
& $w[\mathrm{~mm}]$ & $0,176(8)$ & $0,194(6)$ \\
& $y_{0}[-]$ & $0,1617(28)$ & $0,117(3)$ \\
\hline & & &
\end{tabular}

Tabelle A.3: Aus Abbildung 3.13 ermittelte Werte für Intensität und Rauschen. Als Unsicherheit wird die Standardabweichung berechnet.

\begin{tabular}{lcccc}
\hline & $t=1 \mathrm{~min}$ & $t=5 \mathrm{~min}$ & $t=10 \mathrm{~min}$ & $t=10 \times 1 \mathrm{~min}$ \\
\hline Intensität [Counts] & $109(17)$ & $510(35)$ & $943(49)$ & $971(199)$ \\
Rauschen [Counts] & $70(12)$ & $329(25)$ & $612(35)$ & $622(131)$ \\
\hline
\end{tabular}


Tabelle A.4: Parameter der Gaußkurven $\left(\mathrm{I}(\mathrm{x})=\mathrm{y}_{\mathrm{0}}+\frac{\mathrm{A}}{w \sqrt{\pi / 2}} e^{-2\left(\frac{x-x_{\mathrm{c}}}{w}\right)^{2}}\right)$, die an die horizontalen Intensitätsprofile des Stickstoffplasmas in Abbildung 3.20 angepasst wurden.

\begin{tabular}{ccc}
\hline Profil & Parameter & Wert \\
\hline ohne Apertur & $x_{c}[\mathrm{~mm}]$ & $2,5970(6)$ \\
& $w[\mathrm{~mm}]$ & $0,3810(13)$ \\
& $A[\mathrm{~mm}]$ & $0,4473(14)$ \\
& $y_{0}[-]$ & $0,0281(5)$ \\
mit Apertur & $x_{c}[\mathrm{~mm}]$ & $2,584(4)$ \\
& $w[\mathrm{~mm}]$ & $0,11(9)$ \\
& $A[\mathrm{~mm}]$ & $0,03(6)$ \\
& $y_{0}[-]$ & $0,78(25)$ \\
\hline
\end{tabular}

Tabelle A.5: Parameter der Funktion $F(x)=\left[A \cdot\left(\frac{J_{1}\left(\pi\left(x-x_{c}\right) \frac{d}{d z}\right)}{\pi\left(x-x_{c}\right) \frac{d}{\lambda z}}\right)^{2}\right] *$ $\left[c+B \cdot e^{-m x^{2}}\right]$, die an das horizontale Profil des Beugungsbildes in Abbildung 3.22 angepasst wurden.

\begin{tabular}{cc}
\hline Parameter & Wert \\
\hline A [-] & $445(1)$ \\
$\chi_{c}[\mathrm{~mm}]$ & $677,01(1)$ \\
$c[-]$ & $0,00020(5)$ \\
$B[-]$ & $0,0219(2)$ \\
$\mathrm{m}\left[\mathrm{mm}^{-2}\right]$ & $-5,35(1)$ \\
\hline
\end{tabular}

Tabelle A.6: Photonenenergie charakteristischer Peaks von Polyimid, Siliziumnitrit, Goethit und elementaren Kupfers sowie deren Pixelposition auf dem Detektor des Spektrometers

\begin{tabular}{lcc}
\hline Probe & Pixel & Photonenergie $[\mathrm{eV}]$ \\
\hline Polyimid & 1433 & 285,2 \\
Polyimid & 1424 & 287,4 \\
Polyimid & 1414 & 289,2 \\
Siliziumnitrid & 1022 & 409,8 \\
Polyimid & 751 & 532,0 \\
Goethit & 503 & 709,5 \\
Goehtit & 489 & 722,5 \\
Kupfer & 300 & 933,0 \\
\hline
\end{tabular}


Tabelle A.7: Parameter des Polynom 5. Grades, dass für die Kalibrierung des Gitterspektrometers zur Umrechnung der Pixel in Photonenenergie verwendet wurde.

\begin{tabular}{cc}
\hline Parameter & Wert \\
\hline$a_{0}[\mathrm{eV}]$ & $1412(28)$ \\
$a_{1}[\mathrm{eV} /$ Pixel $]$ & $-1,86(23)$ \\
$a_{2}\left[\mathrm{eV} /\right.$ Pixel $\left.^{2}\right]$ & $7,1(64) \cdot 10^{-4}$ \\
$a_{3}\left[\mathrm{eV} /\right.$ Pixel $\left.^{3}\right]$ & $8,3(89) \cdot 10^{-7}$ \\
$a_{4}\left[\mathrm{eV} /\right.$ Pixel $\left.^{4}\right]$ & $-9,3(58) \cdot 10^{-10}$ \\
$a_{5}\left[\mathrm{eV} /\right.$ Pixel $\left.^{5}\right]$ & $2,6(14) \cdot 10^{-13}$ \\
\hline
\end{tabular}

Tabelle A.8: Parameter der an die optischen Dichten in Abbildung 4.14 angepassten Funktionen.

\begin{tabular}{ccc}
\hline Photonenenergie & Parameter & Wert \\
\hline $282,0 \mathrm{eV}$ & $k_{1}\left[\mathrm{~nm}^{-1}\right]$ & $1,19(3) \cdot 10^{-3}$ \\
$284,7 \mathrm{eV}$ & $k_{2}\left[\mathrm{~nm}^{-1}\right]$ & $2,28(9) \cdot 10^{-3}$ \\
& $k_{3}\left[\mathrm{~nm}^{-2}\right]$ & $-4,7(8) \cdot 10^{-7}$ \\
$288,7 \mathrm{eV}$ & $k_{4}\left[\mathrm{~nm}^{-1}\right]$ & $3,96(11) \cdot 10^{-3}$ \\
& $k_{5}\left[\mathrm{~nm}^{-2}\right]$ & $-1,6(10) \cdot 10^{-7}$ \\
\hline
\end{tabular}

Tabelle A.9: Parameter der an die optischen Dichten in Abbildung 4.16 angepassten linearen Funktionen

\begin{tabular}{ccc}
\hline Photonenenergie & Parameter & Wert \\
\hline $517,1 \mathrm{eV}$ & $\mathrm{m}_{1}\left[\mathrm{~nm}^{-1}\right]$ & $4,04(15) \cdot 10^{-3}$ \\
$531,1 \mathrm{eV}$ & $m_{2}\left[\mathrm{~nm}^{-1}\right]$ & $7,78(18) \cdot 10^{-3}$ \\
$540,4 \mathrm{eV}$ & $m_{3}\left[\mathrm{~nm}^{-1}\right]$ & $7,91(15) \cdot 10^{-3}$ \\
$695,1 \mathrm{eV}$ & $m_{4}\left[\mathrm{~nm}^{-1}\right]$ & $5,50(8) \cdot 10^{-3}$ \\
$707,4 \mathrm{eV}$ & $m_{5}\left[\mathrm{~nm}^{-1}\right]$ & $8,8(2) \cdot 10^{-3}$ \\
$722,5 \mathrm{eV}$ & $m_{6}\left[\mathrm{~nm}^{-1}\right]$ & $7,4(1) \cdot 10^{-3}$ \\
\hline
\end{tabular}


Tabelle A.10: Fitparameter der Gaußkurven $\left(\mathrm{OD}(\mathrm{E})=y_{\mathrm{o}}+\frac{\mathrm{A}}{w \sqrt{\pi / 2}} e^{-2\left(\frac{\mathrm{E}-\mathrm{x}_{\mathrm{c}}}{w}\right)^{2}}\right)$, die an die NEXAFS-Spektren von Hämatit und Goethit an der Sauerstoff-KKante (siehe Abb. 4.19) angepasst wurden.

\begin{tabular}{cccccc}
\hline \multirow{2}{*}{ Probe } & Parameter & \multicolumn{4}{c}{ Wert } \\
& & Peak 1 & Peak 2 & Peak 3 & Peak 4 \\
\hline \multirow{2}{*}{ Hämatit } & $x_{\mathrm{c}}[\mathrm{eV}]$ & $531,0(3)$ & $532,2(5)$ & $537,4(2)$ & $541,6(4)$ \\
& $\mathrm{A}[\mathrm{eV}]$ & $0,6(9)$ & $1,8(10)$ & $0,8(7)$ & $8,0(13)$ \\
& $w[\mathrm{eV}]$ & $1,1(5)$ & $1,9(6)$ & $2,5(10)$ & $7,0(11)$ \\
& $y_{0}[-]$ & $0,083(14)$ & $0,083(14)$ & $0,083(14)$ & $0,083(14)$ \\
\hline \multirow{6}{*}{ Goethit } & $x_{\mathrm{c}}[\mathrm{eV}]$ & $531,0(2)$ & $532,5(3)$ & $537,3(2)$ & $541,9(12)$ \\
& $\mathrm{A}[\mathrm{eV}]$ & $0,6(4)$ & $1,5(5)$ & $2(3)$ & $8(4)$ \\
& $w[\mathrm{eV}]$ & $1,3(3)$ & $1,9(4)$ & $3,7(16)$ & $7(3)$ \\
& $y_{0}[-]$ & $0,04(1)$ & $0,04(1)$ & $0,04(1)$ & $0,04(1)$ \\
\hline
\end{tabular}




\section{NATURKONSTANTEN}

$\begin{array}{lll}\text { Symbol } & \text { Wert und Einheit } & \text { Bedeutung } \\ \mathrm{a}_{0} & 0,52917721067(12) \cdot 10^{-10} \mathrm{~m} & \text { Bohr'scher Radius } \\ \mathrm{c}_{0} & 299792458 \mathrm{~m} \mathrm{~s}^{-1} & \text { Lichtgeschwindigkeit } \\ \mathrm{e} & 1,6021766208(98) \cdot 10^{-19} \mathrm{C} & \text { Elementarladung } \\ \mathrm{e} & 2,71828 \ldots & \text { Euler'sche Zahl } \\ \epsilon_{0} & 8,854187817 \ldots \cdot 10^{-12} \mathrm{~F} / \mathrm{m} & \text { elektrische Feldkonstante } \\ \hbar & 6,582119514(40) \cdot 10^{-16} \mathrm{eV} \mathrm{s} & \text { Planck-Konstante } \\ \mathrm{k}_{\mathrm{B}} & 8,6173303(50) \cdot 10^{-5} \mathrm{eV} / \mathrm{K} & \text { Boltzmann-Konstante } \\ \mathrm{m}_{e} & 9,10938356(11) \cdot 10^{-31} \mathrm{~kg} & \text { Ruhemasse Elektron } \\ \mathrm{m}_{e} \mathrm{c}_{0}{ }^{2} & 0,51099906(15) \mathrm{MeV}^{\prime} & \text { Ruheenergie Elektron } \\ \pi & 3,1415926 \ldots & \text { Kreiszahl } \\ \mathrm{r}_{e} & 2,8179403227(19) \cdot 10^{-15} \mathrm{~m} & \text { klassischer Elektronenradius } \\ \mathrm{R}_{\infty} & 10973731,568508(65) \mathrm{m}^{-1} & \text { Rydberg-Konstante } \\ \sigma & 5,670367(13) \cdot 10^{-8} \mathrm{~W} /\left(\mathrm{m}^{2} \mathrm{~K}^{4}\right) & \text { Stefan-Boltzmann-Konstante }\end{array}$


Abkürzung Bedeutung

Abb.

Abbildung

Abs.

Abschnitt

CCD

charge-coupled device

CDI

coherent diffractive imaging

DESY

Deutsches Elektronen-Synchrotron

ESRF

European Synchrotron Radiation Facility

FLASH

Freie-Elektronen-Laser in Hamburg

HHG

high-harmonic generation

Kap.

Kapitel

LCLS

Linac Coherent Light Source

MTF

modulation transfer function

NEXAFS

near-edge x-ray absorption fine structure

NSLS

National Synchrotron Light Source

PALM

photoactivated localisation microscopy

PETRA III

Positron-Elektron-Tandem-Ring-Anlage III

Ref.

Referenz

SNR

Signal-Rausch-Verhältnis

STED

stimulated emission depletion

STORM

stochastic optical reconstruction microscopy

Tab.

Tabelle

X-FEL

X-Ray Freie-Elektronen-Laser

$\mathrm{ZP}$

Zonenplatte 


\section{ABBILDUNGSVERZEICHNIS}

2.I Spitzen- und mittlere Brillanz ausgewählter Röntgenquellen .

2.2 Synchrotronstrahlung und zugehöriges Spektrum eines Ringbeschleunigers, Wigglers und Undulators . . . . . . . . . .

2.3 Strahlungsleistung als Funktion der im Undulator zurückgelegten Wegstrecke nach dem SASE-Prinzip und Strukturierung der Elektronendichte . . . . . . . . . . . . . . 10

2.4 Exemplarisches Spektrum bei der Erzeugung hoher Harmonischer in Edelgasen . . . . . . . . . . . . . II

2.5 Mechanismen zur Erzeugung freier Elektronen . . . . . . . . . 12

2.6 (a) Trajektorien der Elektronen, die zum Zeitpunkt $t=0$ mit einer Phasenverschiebung $\varphi$ im elektrischen Feld des Laserpulses $(\lambda=800 \mathrm{~nm})$ beschleunigt werden. (b) Kinetische Energie der Elektronen zum Zeitpunkt der Rekombination mit dem Ausgangsion als Funktion der Phase $\varphi . . . . . . .14$

2.7 Emissionsspektrum eines heißen, dichten Plasmas . . . . . . . 17

2.8 Elektronendichteverlauf eines laserinduzierten Plasmas . . . . 20

2.9 Schematische Darstellung und Foto des Aufbaus zur Erzeugung weicher Röntgenstrahlung . . . . . . . . . . 23

2.10 Emissionsspektren verschiedener Gase . . . . . . . . . . . . 25

2.I1 Emissionsspektren von Stickstoff und Krypton für verschiedene Pulslängen . . . . . . . . . . . . . . . . 25

2.12 Vergleich zwischen gemessenen und berechneten Argonspek-

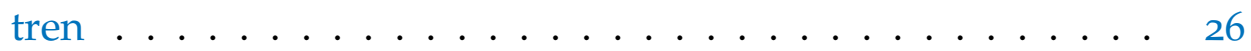

2.13 Emissionsspektren von Stickstoff und Krypton für verschiedene Pulsenergien des Pikosekundenlasers . . . . . . . . . 28

2.14 Anteil der absorbierten Pulsenergie in Stickstoff und Krypton für Nano- und Pikosekundenlaser . . . . . . . . . . . . . . . 29

2.15 Emissionsspektren von Krypton in Abhängigkeit der tatsächlich absorbierten Energie Qabs von Nano- und Pikosekundenlaser ......................... 30

2.16 Intensität von Stickstoff- und Kryptonplasma in Abhängigkeit vom Gasdruck . . . . . . . . . . . . . . 31

2.17 Mikroskopbild eines durch Pikosekundenlaserstrahlung beschädigten Düsenplättchens . . . . . . . . . . . . . . 32

2.18 Schematische Darstellung des Aufbaus zur Plasmacharakterisierung unter $120^{\circ}$ und $30^{\circ}$ zum einfallenden Laserstrahl und entsprechende Lochkamerabilder der Kryptonplasmen . . . .

2.19 Lage und Standardabweichung der Schwerpunkte von 100 Kryptonplasmen unter einem Winkel von $30^{\circ}$ bzw. 120 $\ldots 34$ 
2.20 Schlierenaufnahme zur Visualisierung des Dichtegradienten und Lochkamerabilder der dazugehörigen Plasmen . . . . . . 36

3.I Eindringtiefe weicher Röntgenstrahlung in Wasser und einem typischen Protein als Funktion der Photonenenergie . . . . . .

3.2 Verlauf von $f_{1}^{0}$ und $f_{2}^{0}$ für Kohlenstoff als Funktion der Photonenenergie . . . . . . . . . . . . . . . 4 42

3.3 Mögliche Testobjekte zur Messung der MTF . . . . . . . . . . 44

3.4 Reflektivität an einer Grenzschicht zwischen Vakuum und Materie als Funktion des normierten Einfallswinkels $\theta / \theta_{c}$ für verschiedene Parameter $\beta / \delta \ldots \ldots \ldots . \ldots . \ldots 48$

3.5 Schematische Darstellung einer Fresnel'schen Zonenplatte . . 49

3.6 Berechnete Wirkungsgrade phasenschiebender Zonenplatten aus verschiedenen Materialien . . . . . . . . . 53

3.7 Schematischer Aufbau eines Transmissions- und Rasterröntgenmikroskops .................... 57

3.8 Schematische Darstellung und Foto des Laborröntgenmikro-

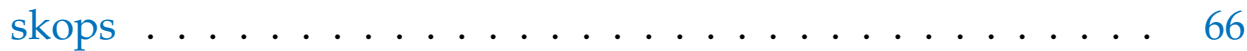

3.9 Mit Aluminium- und Titanfilter gemessenes Emissionsspektrum von Stickstoff. . . . . . . . . . . . . . . . . 66

3.10 Skizze mit Spezifikationen des Kondensorspiegels . . . . . . . 67

3.II Intensitätsverteilung entlang der optischen Achse hinter dem Kondensorspiegel . . . . . . . . . . . . . . 68

3.12 Intensitätsverteilung im Fokus des Kondensorspiegels . . . . 69

3.13 Röntgenmikroskopische Aufnahmen eines Kupfergitters . . 72

3.14 Röntgenmikroskopische Aufnahmen eines Siemenssterns im Hell- und Dunkelfeldontrast . . . . . . . . . . . . . . 74

3.15 Röntgenmikroskopische Aufnahmen eines Siemenssterns für verschiedene Vergrößerungen und Belichtungszeiten . . . . . 75

3.16 Röntgenmikroskopische Aufnahme einer Zonenplatte . . . . . 76

3.17 Kontrast als Funktion der Raumfrequenz . . . . . . . . . . . . 77

3.18 Röntgenmikroskopische Aufnahmen unterschiedlicher biologischer und geologischer Objekte . . . . . . . . . . . 78

3.19 Röntgen- und lichtmikroskopische Aufnahmen verschiedener Algen ..................... . . 80

3.20 Lochkameraaufnahme und horizontale Intensitätsprofile des Stickstoffplasmas ohne und mit Lochblende . . . . . . . . . 82

3.21 Kohärenzgrad der Plasmaquelle berechnet nach Van-CittertZernike-Theorem ................. 83

3.22 Schematische Darstellung des Aufbaus zur kohärenten Bildgebung und Beugungsbild der $10 \mu \mathrm{m}$-Lochblende . . . . . . .

3.23 Horizontaler Querschnitt durch das Beugungsbild der $10 \mu \mathrm{m}$ Lochblende sowie Fitfunktionen für vollständige und partielle Kohärenz . . . . . . . . . . . . . . . . . . 84 
3.24 Kohärenzgrad der Plasmaquelle berechnet nach Van-CittertZernike-Theorem und ermittelt aus Beugungsbild einer Lochblende. . . . . . . . . . . . . . . . .

4.I Übersicht der Elemente, die an der K- bzw. L2-Kante im Energiebereich von 250 bis $1250 \mathrm{eV}$ für die NEXAFS-Spektros-kopie zugänglich sind. . . . . . . . . . . . . .

4.2 Schematische Darstellung des Potenzials für ein zweiatomiges Molekül und des K-Schalen-Absorptionsspektrums mit charakteristischer Feinstruktur . . . . . . . . . . 91

4.3 Schematische Darstellung und Foto des Laborröntgenspektrometers . . . . . . . . . . . . . . . . . 95

4.4 Skizze mit Spezifikationen des Reflexionsgitters . . . . . . . . 96

4.5 Schematische Darstellung und Foto der Probenkammer für Atmosphärendruck. . . . . . . . . . . . . .

4.6 Anhand der Gitterparameter und Pixelbreite des CCD-Chips berechnetes und aus Halbwertsbreite der Spektrallinie von Stickstoff bei $E=430 \mathrm{eV}$ experimentell bestimmtes Auflösungsvermögen des Röntgenspektrometers . . . . . . . . . . 100

4.7 Berechnete Brennebenen des Reflexionsgitters in Abhängigkeit des Einfallswinkels . . . . . . . . . . . . . 100

4.8 Intensität der Stickstofflinie bei $E=430 \mathrm{eV}$ und Auflösungsvermögen des Spektrometers in Abhängigkeit von der Breite des Eintrittsspalts . . . . . . . . . . . . . . 102

4.9 Spektrallinie des Stickstoffs bei $E=430 \mathrm{eV}$ integriert über unterschiedliche Anzahlen von Pulsen für $100 \mu \mathrm{m}$ bzw. $15 \mu \mathrm{m}$ breiten Eintrittsspalt . . . . . . . . . . . . . 103

4.10 Spektrallinie des Stickstoffs bei $E=430 \mathrm{eV}$ und daraus ermittelter Wert des Auflösungsvermögens mit und ohne Quell-

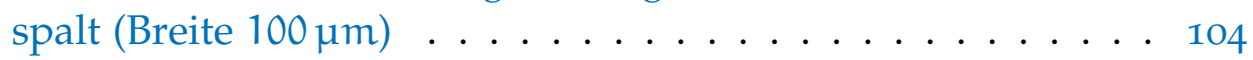

4.II Mikroskopaufnahme des Schichtsilikats CCa-2 Chlorit und einer $200 \mathrm{~nm}$ dicken Agar-100-Probe auf $\mathrm{Si}_{3} \mathrm{~N}_{4}$-Membran . . . 105

4.12 Strukturformel eines typischen Epoxidharzes . . . . . . . . . 105

4.13 NEXAFS-Spektren von Agar-100 (Epoxidharz) verschiedener Probendicke an der Kohlenstoff-K-Kante . . . . . . . . . . . . 106

4.14 Optische Dichte der Agar-100-Proben (links) und Abhängigkeit der optischen Dichte von der Probendicke bei „unvollständiger Absorption" (rechts) . . . . . . . . . . . . 107

4.15 NEXAFS-Spektren von Hämatit verschiedener Probendicken an der Sauerstoff-K- und Eisen-L,2-Kante . . . . . . . . . 109

4.16 Optische Dichte der Hämatit-Proben vor, am 1. sowie 2. Peak der Sauerstoff-K-Kante und vor, am L3-sowie $\mathrm{L}_{2}$-Peak der Eisen-L-Kante aufgetragen gegen die Probendicke . . . . . . . 109

4.17 Vereinfachtes Termschema von Eisenoxiden (z. B. Goethit und Hämatit) sowie Kristallstruktur von Goethit . . . . . . . . . I10 
4.18 NEXAFS-Spektren verschiedener Eisenminerale und des Silikats CCa-2 Chlorit an der Sauerstoff-K-Kante gemessen mit dem Laborröntgenspektrometer und am Synchrotron . . . . . III

4.19 NEXAFS-Spektrum von Hämatit und Goethit an der Sauerstoff-K-Kante sowie daran angepasste Funktion bestehend aus vier Gaußkurven . . . . . . . . . . . . . . . . . . 112

4.20 NEXAFS-Spektren unterschiedlicher Eisenminerale und des Schichtsilikats CCa-2 Chlorit an der Eisen-L3,2-Kante, gemessen mit Laborröntgenspektrometer und am Synchrotron . . . 114

4.21 NEXAFS-Spektrum von Pyrit an der Eisen-L3,2-Kante gemessen mit Laborröntgenspektrometer und am Synchrotron . . . I16

4.22 Emissionsspektren von Krypton und NEXAFS-Spektren von $\mathrm{Si}_{3} \mathrm{~N}_{4}$ (Dicke $100 \mathrm{~nm}$ ) an der Stickstoff-K-Kante mit und ohne

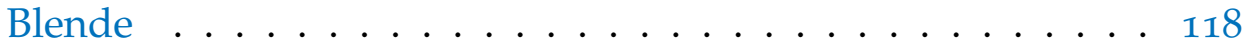

4.23 NEXAFS-Spektren von Goethit und Ferrihydrit an der Sauerstoff-K- und Eisen-L3,2-Kante mit und ohne Blende . . . . . 119

4.24 Emissionsspektren von Krypton, aufgenommen für verschiedene Bedingungen (Vakuum sowie Helium bzw. Luft bei Atmosphärendruck) in der Probenkammer . . . . . . . . . . . 121

4.25 NEXAFS-Spektren von $\mathrm{KMnO}_{4}$, aufgenommen mit Probe im Vakuum (10000 Pulse) sowie in Helium- bzw. Luftatmosphä-

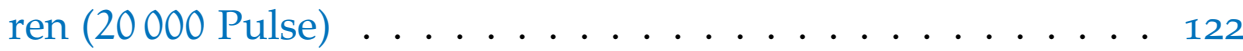

4.26 NEXAFS-Spektren von Calciumchlorid $\left(\mathrm{CaCl}_{2} \cdot \mathrm{H}_{2} \mathrm{O}\right)$ an der Calcium-L- und Sauerstoff-K-Kante, aufgenommen für verschiedene Bedingungen (Vakuum, 10000 Pulse sowie Helium bzw. Luft bei Atmosphärendruck, 20000 Pulse) in der Probenkammer ... . . . . . . . . . . . . 123

5.I Darstellung zur Berechnung eines NEXAFS-Spektrums aus Röntgenmikroskopbildern, aufgenommen bei verschiedenen Energien im Bereich der Absorptionskante . . . . . . . . 128

5.2 Skizzen möglicher Laboraufbauten für die Spektromikroskopie 129

5.3 Emissionsspektren von Neon, Sauerstoff und Stickstoff und Transmission einer $215 \mathrm{~nm}$ dicken Titanfolie zwischen 1,2 $\mathrm{nm}$

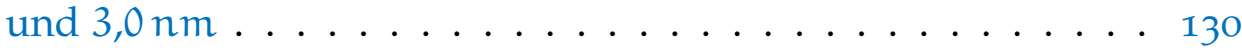

5.4 Mikroskopaufnahme einer $10 \mu \mathrm{m}$ Lochblende, aufgenommen bei $2,48 \mathrm{~nm}$ und $2,88 \mathrm{~nm} \ldots \ldots \ldots$. . . . . . . . 131

5.5 Mikroskopaufnahme einer zum Teil mit Titanfolie bedeckten $\mathrm{Si}_{3} \mathrm{~N}_{4}$-Membran, aufgenommen bei 2,48 $\mathrm{nm}$ und 2,88 nm . . . 132 
TABELLENVERZEICHNIS

2.1 Parameter der eingesetzten Nano- bzw. Pikosekundenlaser .

2.2 Anteile der Elektronentemperaturen $T_{e}$ und Elektronendichten $n_{e}$ zur Berechnung der Argonspektren . . . . . . . . .

2.3 Vergleich charakteristischer Größen des Kryptonplasmas, gemessen mit Lochkamera unter $120^{\circ}$ und $30^{\circ}$ zum einfallenden Laserstrahl . . . . . . . . . . . . . . . . . . . . .

2.4 Vergleich charakteristischer Größen der beiden Plasmen, aufgenommen nur mit Krypton sowie Krypton und Stickstoff zusammen . . . . . . . . . . . . . . .

3.I Charakteristische Größen Fresnel'scher Zonenplatten für die hochauflösende Abbildung . . . . . . . . . . . 50

3.2 Parameter der verwendeten Zonenplatten . . . . . . . . 71

4.I Energie der vier durch Gaußfit ermittelten Peaks an der Sauerstoff-K-Kante von Hämatit sowie Goethit und dazugehörige elektronische Übergänge . . . . . . . . . . . . . . . . . I13

4.2 Energie der Peaks L3,2 von Hämatit, Ferrihydrit, CCa-2 Chlorit und Pyrit . . . . . . . . . . . . . . I15

A.I Übersicht der wichtigsten im Rahmen dieser Arbeit genutzten Messgeräte, ihrer Anwendungen und technischen Kenngrößen . . . . . . . . . . . . . . . . . . . 139

A.2 Fitparameter der Lorentzfunktionen, die an die Intensitätsprofile im Fokus des elliptischen Kondensorspiegels (siehe Abb. 3.12) angepasst wurden. . . . . . . . . . . 140

A.3 Aus Abbildung 3.13 ermittelte Werte für Intensität und Rauschen . . . . . . . . . . . . . . . . I40

A.4 Parameter der Gaußkurven, die an die horizontalen Profile des Stickstoffplasmas in Abbildung 3.20 angepasst wurden. . I4I

A.5 Parameter der Funktion, die an das horizontale Profil des Beugungsbildes in Abbildung 3.22 angepasst wurden. . . . . I41

A.6 Photonenenergie charakteristischer Peaks von Polyimid, Siliziumnitrit, Goethit und elementaren Kupfers sowie deren Pixelposition auf dem Detektor des Spektrometers . . . . . . I41

A.7 Parameter des Polynom 5. Grades, dass für die Kalibrierung des Gitterspektrometers zur Umrechnung der Pixel in Photonenenergie verwendet wurde. . . . . . . . . . . . . 142

A.8 Parameter der an die optischen Dichten in Abbildung 4.14 angepassten Funktionen . . . . . . . . . . . . . 142

A.9 Parameter der an die optischen Dichten in Abbildung 4.16 angepassten linearen Funktionen . . . . . . . . 142 
A.1o Fitparameter der Gaußkurven, die an die NEXAFS-Spektren von Hämatit und Goethit an der Sauerstoff-K-Kante (siehe Abb. 4.19) angepasst wurden. . . . . . . . . . . . . 143 
TABELLENVERZEICHNIS 
[1] CRoft, W. J.: Under the Microscope: A Brief History of Microscopy. World Scientific Publishing, 2005. - ISBN 981-02-3781-2

[2] Авве, E.: Beiträge zur Theorie des Mikroskops und der mikroskopischen Wahrnehmung. In: Archiv für Mikroskopische Anatomie 9 (1873), Dezember, Nr. 1, S. 413-418. http://dx.doi.org/10.1007/ BF02956173. - DOI 10.1007/BF02956173

[3] Hell, S. ; Wichmann, J.: Breaking the diffraction resolution limit by stimulated emission: stimulated-emission-depletion fluorescence microscopy. In: Optics Letters 19 (1994), Juni, Nr. 11, S. 780. http://dx.doi.org/10.1364/0L.19.000780. - DOI 10.1364/OL.19.000780

[4] Betzig, E. ; Patterson, G. ; Sougrat, R. ; Lindwasser, O. ; Olenych, S. ; Bonifacino, J. ; Davidson, M. ; LippincottSchwartz, J. ; Hess, H.: Imaging Intracellular Fluorescent Proteins at Nanometer Resolution. In: Science 313 (2006), September, Nr. 5793, S. 1642-1645. http: //dx. doi . org/10. 1126/science. 1127344. - DOI 10.1126/science.1127344

[5] Rust, M. ; Bates, M. ; Zhuang, X.: Sub-diffraction-limit imaging by stochastic optical reconstruction microscopy (STORM). In: Nature Methods 3 (2006), August, Nr. 10, S. 793-796. http: //dx.doi.org/10.1038/NMETH929. - DOI 10.1038/NMETH929

[6] Aтtwood, D.: Soft X-Rays and Extreme Ultraviolet Radiation: Principles and Applications. Cambridge University Press, 1999. - ISBN 0521652146

[7] FAHRni, C.: Biological applications of X-ray fluorescence microscopy: exploring the subcellular topography and speciation of transition metals. In: Current Opinion in Chemical Biology 11 (2007), April, Nr. 2, S. 121-127. http://dx.doi.org/10.1016/j .cbpa.2007.02. 039. - DOI 10.1016/j.cbpa.2007.02.039

[8] Song, C. ; Jiang, H. ; Mancuso, A. ; Amirbekian, B. ; Peng, L. ; Sun, R. ; Shah, S. ; Zhou, H. ; Ishikawa, T. ; Miao, J.: Quantitative Imaging of Single, Unstained Viruses with Coherent X Rays. In: Physical Review Letters 101 (2008), Oktober, Nr. 15. http://dx.doi.org/10.1103/PhysRevLett.101.158101. - DOI 10.1103/PhysRevLett.101.158101 
[9] Giewekemeyer, K. ; Krüger, S. ; Kalbfleisch, S. ; Bartels, M. ; Beta, C. ; SAlditT, T.: X-ray propagation microscopy of biological cells using waveguides as a quasipoint source. In: Physical Review A 83 (2011), Februar, Nr. 2. http://dx.doi.org/10.1103/ PhysRevA. 83.023804. - DOI 10.1103/PhysRevA.83.023804

[1o] Bartels, M. ; Krenkel, M. ; Haber, J. ; Wilke, R. ; Salditt, T.: X-Ray Holographic Imaging of Hydrated Biological Cells in Solution. In: Physical Review Letters 114 (2015), Januar, Nr. 4. http://dx.doi.org/10.1103/PhysRevLett.114.048103. - DOI 10.1103/PhysRevLett.114.048103

[11] Shapiro, D. ; Thibault, P. ; Beetz, T. ; Elser, V. ; Howells, M. ; Jacobsen, C. ; Kirz, J. ; Lima, E. ; Miao, H. ; Neiman, A. ; SayRE, D.: Biological imaging by soft x-ray diffraction microscopy. In: Proceedings of the National Academy of Sciences 102 (2005), Oktober, Nr. 43, S. 15343-15346. http://dx. doi.org/10.1073/pnas. 0503305102. - DOI 10.1073/pnas.0503305102

[12] Niemann, B. ; Rudolph, D. ; Schmahl, G.: X-ray microscopy with synchrotron radiation. In: Applied Optics 15 (1976), August, Nr. 8, S. 1883. http://dx.doi.org/10.1364/A0.15.001883. - DOI 10.1364/AO.15.001883

[13] Kaulich, B. ; Thibault, P. ; Gianoncelli, A. ; Kiskinova, M.: Transmission and emission $\mathrm{x}$-ray microscopy: operation modes, contrast mechanisms and applications. In: Journal of Physics: Condensed Matter 23 (2011), Februar, Nr. 8, S. 083002. http://dx. doi.org/10.1088/0953-8984/23/8/083002. - DOI 10.1088/0953$8984 / 23 / 8 / 083002$

[14] Meyer-Ilse, W. ; Hamamoto, D. ; Nair, A. ; Lelievre, S. ; Denbeaux, G. ; Johnson, L. ; Pearson, A. ; Yager, D. ; Legros, M. ; LARABELL, C.: High resolution protein localization using soft Xray microscopy. In: Journal of Microscopy 201 (2001), März, Nr. 3, S. 395-403. http://dx.doi.org/10.1046/j.1365-2818.2001.00845. x. - DOI 10.1046/j.1365-2818.2001.00845.x

[15] Parkinson, D. ; McDermott, G. ; Etkin, L. ; Gros, M. L. ; LARABELL, C.: Quantitative 3-D imaging of eukaryotic cells using soft X-ray tomography. In: Journal of Structural Biology 162 (2008), Juni, Nr. 3, S. 380-386. http://dx.doi.org/10.1016/j . j sb. 2008. 02.003. - DOI 10.1016/j.jsb.2008.02.003

[16] Gros, M. L. ; McDermott, G. ; Cinquin, B. ; Smith, E. ; Do, M. ; Chao, W. ; Naulleau, P. ; Larabell, C.: Biological soft 
X-ray tomography on beamline 2.1 at the Advanced Light Source. In: Journal of Synchrotron Radiation 21 (2014), Oktober, Nr. 6, S. 1370-1377. http://dx.doi.org/10.1107/S1600577514015033. DOI 10.1107/S1600577514015033

[17] Zschech, E. ; Yun, W. ; Schneider, G.: High-resolution X-ray imaging - a powerful nondestructive technique for applications in semiconductor industry. In: Applied Physics A 92 (2008), Mai, Nr. 3, S. 423-429. http: //dx. doi .org/10.1007/s00339-008-4551-x. DOI 10.1007/so0339-008-4551-x

[18] Kasai, S. ; Fischer, P. ; Im, M.-Y. ; Yamada, K. ; Nakatani, Y. ; Kobayashi, K. ; Kohno, H. ; Ono, T.: Probing the Spin Polarization of Current by Soft X-Ray Imaging of Current-Induced Magnetic Vortex Dynamics. In: Physical Review Letters 101 (2008), Dezember, Nr. 23. http://dx.doi.org/10.1103/PhysRevLett.101.237203. DOI 10.1103/PhysRevLett.101.237203

[19] Groot, F. de ; Smit, E. de ; Schooneveld, M. van ; Aramburo, L. ; Weckhursen, B.: In-situ Scanning Transmission X-Ray Microscopy of Catalytic Solids and Related Nanomaterials. In: ChemPhysChem 11 (2010), März, Nr. 5, S. 951-962. http://dx.doi.org/ 10.1002/cphc. 200901023. - DOI 10.1002/cphc.200901023

[20] Kapitel 8. In: Cosmidis, J. ; BenzerarA, K.: Soft $x$-ray scanning transmission spectromicroscopy. CRC Press, 2014. - ISBN 978-1$4665-1837-7$, S. 115-133

[21] Berglund, M. ; Rymell, L. ; Peuker, M. ; Wilhein, T. ; Hertz, H.: Compact water-window transmission X-ray microscopy. In: Journal of Microscopy 197 (2000), März, Nr. 3, S. 268-273. http://dx.doi.org/10.1046/j.1365-2818.2000.00675.x. - DOI 10.1046/j.1365-2818.2000.00675.x

[22] Benk, M. ; Bergmann, K. ; Schäfer, D. ; Wilhein, T.: Compact soft $x$-ray microscope using a gas-discharge light source. In: Optics Letters 33 (2008), Oktober, Nr. 20, S. 2359. http://dx. doi.org/10. 1364/0L.33.002359. - DOI 10.1364/OL.33.002359

[23] Allain, J. ; Hassanein, A. ; Allain, M. ; Heuser, B. ; Nieto, M. ; Снговак, C. ; Rokusek, D. ; Rice, B.: Xe ${ }^{+}$-irradiation effects on multilayer thin-film optical surfaces in EUV lithography. In: Nuclear Instruments and Methods in Physics Research Section B: Beam Interactions with Materials and Atoms 242 (2006), Januar, Nr. 1-2, S. 520-522. http://dx.doi.org/10.1016/j.nimb.2005.08.188. DOI 10.1016/j.nimb.2005.08.188 
[24] Fiedorowicz, H. ; Bartnik, A. ; Patron, Z. ; Parys, P.: X-ray emission from laser-irradiated gas puff targets. In: Applied Physics Letters 62 (1993), Mai, Nr. 22, S. 2778-2780. http://dx.doi.org/ 10.1063/1.109232. - DOI 10.1063/1.109232

[25] Kranzusch, S. ; MAnN, K.: Spectral characterization of EUV radiation emitted from a laser-irradiated gas puff target. In: Optics Communications 200 (2001), Dezember, Nr. 1-6, S. 223230. http : //dx.doi.org/10.1016/S0030-4018(01)01639-X. - DOI 10.1016/Sо030-4018(о1)о1639-X

[26] Ретн, C.: XUV-Laserplasmaquellen für die Absorptions-Spektroskopie und zeitaufgelöste Röntgenbeugung, Georg-August-Universität Göttingen, Dissertation, Oktober 2008

[27] Peth, C. ; Barkusky, F. ; MAnN, K.: Near-edge x-ray absorption fine structure measurements using a laboratory-scale XUV source. In: Journal of Physics D: Applied Physics 41 (2008), April, Nr. 10, S. 105202. http://dx.doi.org/10.1088/0022-3727/41/10/105202. DOI 10.1088/0022-3727/41/10/105202

[28] Nováková, E. ; Mitrea, G. ; Peth, C. ; Thieme, J. ; Mann, K. ; SAlditT, T.: Solid supported multicomponent lipid membranes studied by x-ray spectromicroscopy. In: Biointerphases 3 (2008), Nr. 2. http://dx.doi.org/10.1116/1.2976445. - DOI $10.1116 / 1.2976445$

[29] Sedlmair, J. ; Gleber, S.-C. ; Peth, C. ; Mann, K. ; Niemeyer, J. ; THIEME, J.: Characterization of refractory organic substances by NEXAFS using a compact X-ray source. In: Journal of Soils and Sediments 12 (2012), Januar, Nr. 1, S. 24-34. http://dx.doi.org/ 10.1007/s11368-011-0385-9. - DOI 10.1007/s11368-011-0385-9

[30] Röntgen, W. C.: Ueber eine neue Art von Strahlen. In: Annalen der Physik 300 (1898), Nr. 1, 1-11. http://dx.doi.org/10.1002/andp. 18983000102. - DOI 10.1002/andp.18983000102. - ISSN 1521-3889

[31] Als-Nielsen, J. ; McMorrow, D.: Elements of Modern X-Ray Physics. John Wiley \& Sons, Ltd, 2001. - ISBN 978-0471-49858-2

[32] Pfeifer, T. ; Spielmann, C. ; Gerber, G.: Femtosecond x-ray science. In: Reports on Progress in Physics 69 (2006), Januar, Nr. 2, S. 443-505. http://dx.doi.org/10.1088/0034-4885/69/2/R04. DOI 10.1088/0034-4885/69/2/Ro4

[33] Ackermann, W. ; Asova, G. ; Ayvazyan, V. ; Azima, A. ; Baboi, N. ; Bähr, J. ; Balandin, V. ; Beutner, B. ; Brandt, A. 
; Bolzmann, A. ; Brinkmann, R. ; Brovko, I. O. ; Castellano, M. ; Castro, P. ; Catani, L. ; Chiadroni, E. ; Choroba, S. ; Cianchi, A. ; Costello, T. J. ; Cubaynes, D. ; Dardis, J. ; Decking, W. ; Delsim-Hashemi, H. ; Delserieys, A. ; Pirro, Di G. ; Dohlus, M. ; Düsterer, S. ; Eckhardt, A. ; Edwards, T. H. ; FaAtz, B. ; Feldhaus, J. ; Flöttmann, K. ; Frisch, J. ; Fröhlich, L. ; Garvey, T. ; Gensch, U. ; Gerth, Ch. ; Görler, M. ; Golubeva, N. ; Grabosch, H.-J. ; Grecki, M. ; Grimm, O. ; Hacker, K. ; Hahn, U. ; Han, H. J. ; Honkavaara, K. ; Hott, T. ; Hüning, M. ; IvanisenKo, Y. ; JaeschKe, E. ; Jalmuzna, W. ; Jezynski, T. ; Kammering, R. ; Katalev, V. ; Kavanagh, K. ; Kennedy, T. E. ; Khodyachykh, S. ; Klose, K. ; Kocharyan, V. ; Körfer, M. ; Kollewe, M. ; Koprek, W. ; Korepanov, S. ; Kostin, D. ; Krassilnikov, M. ; Kube, G. ; Kuhlmann, M. ; Lewis, S. C. L. C. L. ; Lilje, L. ; LimberG, T. ; LipKa, D. ; Löhl, F. ; LUna, H. ; Luong, M. ; Martins, M. ; Meyer, M. ; Michelato, P. ; Miltchev, V. ; Möller, D. W. ; Monaco, L. ; Müller W. F., O. ; Napieralski, O. ; Napoly, O. ; Nicolosi, P. ; Nölle, D. ; Nunez, T. ; Oppelt, A. ; Pagani, C. ; Paparella, R. ; Pchalek, N. ; Pedregosa-Gutierrez, J. ; Petersen, B. ; Petrosyan, B. ; Petrosyan, G. ; Petrosyan, L. ; Pflüger, J. ; E., Plönjes ; Poletto, L. ; Pozniak, K. ; Prat, E. ; Proch, D. ; Pucyk, P. ; Radcliffe, P. ; Redlin, H. ; Rehlich, K. ; Richter, M. ; Roehrs, M. ; Roensch, J. ; Romaniuk, R. ; Ross, M. ; Rossbach, J. ; Rybnikov, V. ; Sachwitz, M. ; Saldin, L. E. ; Sandner, W. ; Schlarb, H. ; Schmidt, B. ; Schmitz, M. ; Schmüser, P. ; Schneider, R. J. ; Schneidmiller, A. E. ; Schnepp, S. ; Schreiber, S. ; Seidel, M. ; Sertore, D. ; Shabunov, V. A. ; Simon, C. ; Simrock, S. ; Sombrowski, E. ; Sorokin, A. A. ; Spanknebel, P. ; Spesyvtsev, R. ; Staykov, L. ; Steffen, B. ; Stephan, F. ; Stulle, F. ; Thom, H. ; TiedtKe, K. ; Tischer, M. ; Toleikis, S. ; Treusch, R. ; Trines, D. ; Tsakov, I. ; Vogel, E. ; Weiland, T. ; Weise, H. ; Wellhöfer, M. ; Wendt, M. ; Will, I. ; Winter, A. ; Wittenburg, K. ; Wurth, W. ; Yeates, P. ; Yurkov, V. M. ; Zagorodnov, I. ; ZAPFE, K.: Operation of a free-electron laser from the extreme ultraviolet to the water window. In: Nature Photonics I (2007), Juni, Nr. 6, 336-342. http://dx. doi.org/10. 1038/nphoton. 2007. 76. - ISSN 1749-4885

[34] Thompson, A. C. (Hrsg.): X-Ray Data Booklet. 3. Ausgabe. Lawrence Berkeley National Laboratory, University of California, Berkeley, California : Center for X-Ray Optics Advanced Light Sources, 2009 http: //xdb. lbl.gov/ 
[35] Suckewer, S. ; Jaeglé, P.: X-Ray laser: past, present, and future. In: Laser Physics Letters 6 (2009), Juni, Nr. 6, S. 411-436. http://dx. doi.org/10. 1002/lapl. 200910023. - DOI 10.1002/lapl.200910023

[36] Macchietto, C. D. ; Benware, B. R. ; Rocca, J. J.: Generation of millijoule-level soft-x-ray laser pulses at a $4-\mathrm{Hz}$ repetition rate in a highly saturated tabletop capillary discharge amplifier. In: Optics Letters 24 (1999), August, Nr. 16, S. 1115. http://dx.doi.org/10. 1364/0L . 24.001115. - DOI 10.1364/OL.24.001115

[37] Janulewicz, K. A. ; Lucianetti, A. ; Priebe, G. ; Nickles, P. V.: Review of state-of-the-art and output characteristics of table-top soft x-ray lasers. In: X-Ray Spectrometry 33 (2004), Juli, Nr. 4, S. 262266. http://dx.doi.org/10.1002/xrs.718. - DOI 10.1002/xrs.718

[38] Alessi, D. ; Wang, Y. ; Luther, B. M. ; Yin, L. ; Martz, D. H. ; Woolston, M. R. ; LiU, Y. ; Berrill, M. ; RoccA, J. J.: Efficient Excitation of Gain-Saturated Sub-9-nm-Wavelength Tabletop SoftX-Ray Lasers and Lasing Down to $7.36 \mathrm{~nm}$. In: Physical Review X I (2011), Dezember, Nr. 2. http://dx. doi.org/10.1103/PhysRevX. 1.021023. - DOI 10.1103/PhysRevX.1.021023

[39] Behling, R.: Modern Diagnostic X-Ray Sources. CRC Press Taylor \& Francis Group, 2016. - ISBN 9781482241327

[40] Imanenko, D. ; Pomeranchuk, I.: On the Maximal Energy Attainable in a Betatron. In: Physical Review 65 (1944), Juni, Nr. 11-12, S. 343-343. http://dx.doi.org/10.1103/PhysRev.65.343. - DOI 10.1103/PhysRev.65.343

[41] Elder, F. R. ; Gurewitsch, A. M. ; Langmuir, R. V. ; Pollock, H. C.: Radiation from Electrons in a Synchrotron. In: Physical Review 71 (1947), Juni, Nr. 11, S. 829-830. http://dx. doi.org/10. 1103/PhysRev.71.829.5. - DOI 10.1103/PhysRev.71.829.5

[42] Wiedemann, H.: Particle Accelerator Physics. 3. Ausgabe. Springer, 2007. - ISBN 9783540490432

[43] Madey, J. M. J.: Stimulated Emission of Bremsstrahlung in a Periodic Magnetic Field. In: Journal of Applied Physics 42 (1971), April, Nr. 5, S. 1906-1913. http://dx.doi.org/10.1063/1.1660466. DOI 10.1063/1.1660466

[44] Deacon, D. A. G. ; Elias, L. R. ; Madey, J. M. J. ; Ramian, G. J. ; Schwettman, H. A. ; Smith, T. I.: First Operation of a FreeElectron Laser. In: Physical Review Letters 38 (1977), April, Nr. 16, S. 892-894. http://dx.doi.org/10.1103/PhysRevLett.38.892. DOI 10.1103/PhysRevLett.38.892 
[45] McNeIL, B.: Free electron lasers: First light from hard X-ray laser. In: Nature Photonics 3 (2009), Juli, Nr. 7, S. 375-377. http://dx. doi. org/10.1038/nphoton. 2009.110. - DOI 10.1038/nphoton.2009.110

[46] McNeil, B. W. J. ; Thompson, N. R.: X-ray free-electron lasers. In: Nature Photonics 4 (2010), November, Nr. 12, S. 814-821. http: //dx.doi.org/10.1038/nphoton.2010.239. - DOI 10.1038/nphoton.2010.239

[47] HuAng, Z. ; Kim, K.-J.: Review of x-ray free-electron laser theory. In: Physical Review Special Topics - Accelerators and Beams 10 (2007), März, Nr. 3, S. 034801. http://dx. doi . org/10. 1103/PhysRevSTAB. 10.034801. - DOI 10.1103/PhysRevSTAB.10.034801

[48] Margaritondo, G. ; Ribic, P. R.: A simplified description of $\mathrm{X}$-ray free-electron lasers. In: Journal of Synchrotron Radiation 18 (2011), Januar, Nr. 2, S. 101-108. http://dx.doi.org/10.1107/ S090904951004896X. - DOI 10.1107/So90904951004896X. - ISSN 0909-0495

[49] Kontratenko, A. M. ; Saldin, E. L.: Generation of coherent radiation by a relativistic electron beam in an ondulator. In: Particle Accelerators 10 (1980), Januar, Nr. 3-4, S. 207-216. - ISSN o031-2460

[50] Tiedtke, K. ; Azima, A. ; Bargen, N. von ; Bittner, L. ; Bonfigt, S. ; Düsterer, S. ; FaAtz, B. ; Frühling, U. ; Gensch, M. ; Gerth, C. ; Guerassimova, N. ; Hahn, U. ; Hans, T. ; HesSe, M. ; HonkavaAr, K. ; Jastrow, U. ; Juranic, P. ; Kapitzki, S. ; Keitel, B. ; Kracht, T. ; KuhlmanN, M. ; Li, W. B. ; Martins, M. ; Núñez, T. ; Plönjes, E. ; Redlin, H. ; SAldin, E. L. ; Schneidmiller, E. A. ; Schneider, J. R. ; Schreiber, S. ; Stojanovic, N. ; Tavella, F. ; Toleikis, S. ; Treusch, R. ; Weigelt, H. ; Wellhöfer, M. ; Wabnitz, H. ; Yurkov, M. V. ; Feldhaus, J.: The soft $x$-ray free-electron laser FLASH at DESY: beamlines, diagnostics and end-stations. In: New Journal of Physics 11 (2009), Nr. 2, o23029. http://stacks.iop.org/1367-2630/11/i=2/a=023029

[51] Saldin, E. L. ; Schneidmiller, E. A. ; Yurkov, M. V.: Statistical and coherence properties of radiation from $x$-ray freeelectron lasers. In: New Journal of Physics 12 (2010), März, Nr. 3, S. 035010. http://dx.doi.org/10.1088/1367-2630/12/3/035010. - DOI 10.1088/1367-2630/12/3/035010

[52] Allaria, E. ; Appio, R. ; Badano, L. ; Barletta, W. A. ; Bassanese, S. ; Biedron, S. G. ; Borga, A. ; Busetto, E. ; Castronovo, D. ; Cinquegrana, P. ; Cleva, S. ; Cocco, D. ; Cornacchia, M. ; Craievich, P. ; Cudin, I. ; D’Auria, G. ; Forno, M. D. 
; Danailov, M.B. ; Monte, R. D. ; Ninno, G. D. ; Delgiusto, P. ; Demidovich, A. ; Mitri, S. D. ; Diviacco, B. ; Fabris, A. ; Fabris, R. ; Fawley, W. ; Ferianis, M. ; Ferrari, E. ; Ferry, S. ; Froehlich, L. ; Furlan, P. ; Gaio, G. ; Gelmetti, F. ; Giannessi, L. ; Giannini, M. ; Gobessi, R. ; Ivanov, R. ; KaRantzoulis, E. ; Lonza, M. ; Lutman, A. ; Mahieu, B. ; Milloch, M. ; Milton, S. V. ; Musardo, M. ; Nikolov, I. ; Noe, S. ; Parmigiani, F. ; Penco, G. ; Petronio, M. ; Pivetta, L. ; Predonzani, M. ; Rossi, F. ; Rumiz, L. ; Salom, A. ; Scafuri, C. ; Serpico, C. ; Sigalotti, P. ; Spampinati, S. ; Spezzani, C. ; Svandrlik, M. ; Svetina, C. ; Tazzari, S. ; Trovo, M. ; Umer, R. ; Vascotto, A. ; Veronese, M. ; Visintini, R. ; Zaccaria, M. ; ZANGRANDO, D. ; ZANGRANDO, M.: Highly coherent and stable pulses from the FERMI seeded free-electron laser in the extreme ultraviolet. In: Nature Photonics 6 (2012), September, Nr. 10, S. 699-704. http://dx.doi.org/10.1038/NPHOTON.2012.233. - DOI 10.1038/NPHOTON.2012.233

[53] Amann, J. ; Berg, W. ; Blank, V. ; Decker, F.-J. ; Ding, Y. ; Emma, P. ; Feng, Y. ; Frisch, J. ; Fritz, D. ; Hastings, J. ; Huang, Z. ; Krzywinski, J. ; Lindberg, R. ; Loos, H. ; Lutman, A. ; Nuhn, H.-D. ; Ratner, D. ; Rzepiela, J. ; Shu, D. ; Shvyd'ko, Yu. ; Spampinati, S. ; Stoupin, S. ; Terentyev, S. ; Trakhtenberg, E. ; Walz, D. ; Welch, J. ; Wu, J. ; ZholENTS, A. ; ZHU, D.: Demonstration of self-seeding in a hard-X-ray free-electron laser. In: Nature Photonics 6 (2012), August, Nr. 10, S. 693-698. http://dx.doi.org/10.1038/NPHOTON.2012.180. - DOI 10.1038/NPHOTON.2012.180

[54] European XFEL - Facts E Figures: Properties of the X-Ray Laser Flashes. http://www.xfel .eu/overview/facts_and_figures/. - aufgerufen am 03.03.2017

[55] Nakajima, K.: Compact X-ray sources: Towards a table-top freeelectron laser. In: Nature Physics 4 (2008), Februar, Nr. 2, S. 92-93. http://dx.doi.org/10.1038/nphys846. - DOI 10.1038/nphys846

[56] Maier, A. R. ; Meseck, A. ; Reiche, S. ; Schroeder, C. B. ; Seggebrock, T. ; Grüner, F.: Demonstration Scheme for a Laser-Plasma-Driven Free-Electron Laser. In: Physical Review X 2 (2012), September, Nr. 3. http://dx.doi.org/10.1103/PhysRevX. 2.031019. - DOI 10.1103/PhysRevX.2.031019

[57] Tilborg, J. van ; Barber, S. K. ; Isono, F. ; Schroeder, C. B. ; Esarey, E. ; Leemans, W. P.: Free-electron lasers driven by laser plasma accelerators. In: AIP Conference Proceedings 1812 (2017), 
März, Nr. 1, S. 020002. http://dx.doi.org/10.1063/1.4975838. DOI 10.1063/1.4975838

[58] Franken, P. A. ; Hill, A. E. ; Peters, C. W. ; Weinreich, G.: Generation of Optical Harmonics. In: Physical Review Letters 7 (1961), August, Nr. 4, S. 118-119. http://dx.doi.org/10.1103/ PhysRevLett .7.118. - DOI 10.1103/PhysRevLett.7.118

[59] McPherson, A. ; Gibson, G. ; Jara, H. ; Johann, U. ; LuK, T. S. ; McIntyre, I. A. ; Boyer, K. ; Rhodes, C. K.: Studies of multiphoton production of vacuum-ultraviolet radiation in the rare gases. In: Journal of the Optical Society of America B 4 (1987), April, Nr. 4, S. 595. http://dx. doi . org/10. 1364/J0SAB . 4.000595. - DOI 10.1364/JOSAB.4.000595

[6o] Ferray, M. ; L'Huillier, A. ; Li, X. F. ; Lompre, L. A. ; MainFrAY, G. ; MANus, C.: Multiple-harmonic conversion of 1064 $\mathrm{nm}$ radiation in rare gases. In: Journal of Physics B: Atomic, Molecular and Optical Physics 21 (1988), Februar, Nr. 3, S. L31L35. http://dx.doi.org/10.1088/0953-4075/21/3/001. - DOI 10.1088/0953-4075/21/3/001

[61] Seres, J. ; Seres, E. ; Verhoef, A. J. ; Tempea, G. ; Streli, C. ; WobrauscheK, P. ; Yakovlev, V. ; Scrinzi, A. ; Spielmann, C. ; KRAUsz, F.: Laser technology: Source of coherent kiloelectronvolt X-rays. In: Nature 433 (2005), Februar, Nr. 7026, S. 596-596. http: //dx.doi.org/10.1038/433596a. - DOI 10.1038/433596a

[62] Paul, P. M. ; Toma, E. S. ; Berger, P. ; Mullot, G. ; Auge, F. ; Balcou, Ph. ; Muller, H. G. ; Agostini, P.: Observation of a Train of Attosecond Pulses from High Harmonic Generation. In: Science 292 (2001), Juni, Nr. 5522, S. 1689-1692. http://dx.doi. org/10.1126/science.1059413. - DOI 10.1126/science.1059413

[63] Midorikawa, K.: High-Order Harmonic Generation and Attosecond Science. In: Japanese Journal of Applied Physics 50 (2011), September, Nr. 9, S. ogooo1. http://dx.doi.org/10.1143/JJAP. 50.090001. - DOI 10.1143/JJAP.50.090001

[64] Silva, F. ; Teichmann, S. M. ; Cousin, S. L. ; Hemmer, M. ; BieGERT, J.: Spatiotemporal isolation of attosecond soft X-ray pulses in the water window. In: Nature Communications 6 (2015), mar, S. 6611. http://dx.doi.org/10.1038/ncomms7611. - DOI 10.1038/ncomms7611

[65] Li, X. F. ; L'Huillier, A. ; Ferray, M. ; Lompré, L. A. ; MainFRAY, G.: Multiple-harmonic generation in rare gases at high laser 
intensity. In: Physical Review A 39 (1989), Juni, Nr. 11, S. 57515761. http://dx.doi.org/10.1103/PhysRevA.39.5751. - DOI 10.1103/PhysRevA.39.5751

[66] Corkum, P. B.: Plasma perspective on strong field multiphoton ionization. In: Physical Review Letters 71 (1993), September, Nr. 13, S. 1994-1997. http://dx.doi.org/10.1103/PhysRevLett.71.1994. - DOI 10.1103/PhysRevLett.71.1994

[67] Kulander, K. C. ; Schafer, K. J. ; Krause, J. L.: Dynamics of Short-Pulse Excitation, Ionization and Harmonic Conversion. Version: 1993. http://dx. doi . org/10 . 1007/978-1-4615-7963-2 10. In: L'Huillier, A. (Hrsg.) ; Piraux, B. (Hrsg.) ; Rzazewski, K. (Hrsg.): Super-Intense Laser-Atom Physics. Springer US, 1993. DOI 10.1007/978-1-4615-7963-2_10. - ISBN 978-1-4615-7963-2, Kapitel 10, S. 95-110

[68] Keldysh, L. V.: Ionization in the Field of a Strong Electromagnetic Wave. In: Journal of Experimental and Theoretical Physics 20 (1965), Mai, Nr. 5, 1307-1314. http://jetp.ac.ru/cgi-bin/dn/e_020_05_ 1307.pdf

[69] Krause, J. L. ; Schafer, K. J. ; Kulander, K. C.: High-order harmonic generation from atoms and ions in the high intensity regime. In: Physical Review Letters 68 (1992), Juni, Nr. 24, S. 35353538. http://dx.doi.org/10.1103/PhysRevLett.68.3535. - DOI 10.1103/PhysRevLett.68.3535

[7o] Christov, I. P. ; Murnane, M. M. ; Kapteyn, H. C.: HighHarmonic Generation of Attosecond Pulses in the "Single-Cycle" Regime. In: Physical Review Letters 78 (1997), Februar, Nr. 7, S. 1251-1254. http://dx.doi.org/10.1103/PhysRevLett.78.1251. DOI 10.1103/PhysRevLett.78.1251

[71] Teichmann, S. M. ; Silva, F. ; Cousin, S. L. ; Hemmer, M. ; BIEgeRT, J.: 0.5-keV Soft X-ray attosecond continua. In: Nature Communications 7 (2016), Mai, S. 11493. http://dx.doi.org/10. 1038/ncomms11493. - DOI 10.1038/ncomms11493

[72] Raith, W. ; Schaefer, C.: Lehrbuch der Experimentalphysik: Elektromagnetismus. Bd. 2. 8. Auflage. Berlin, New York: Walter De Gruyter Inc, 1999. - ISBN 3110160978

[73] Bennett, W. H.: Magnetically Self-Focussing Streams. In: Physical Review 45 (1934), Juni, Nr. 12, S. 890-897. http://dx. doi.org/10. 1103/PhysRev.45.890. - DOI 10.1103/PhysRev.45.890 
[74] Bergmann, K. ; Küpper, F. ; BenK, M.: Soft x-ray emission from a pulsed gas discharge in a pseudosparklike electrode geometry. In: Journal of Applied Physics 103 (2008), Juni, Nr. 12, S. 123304. http: //dx.doi.org/10.1063/1.2940786. - DOI 10.1063/1.2940786

[75] Powell, K. ; Duffy, G. ; Choi, P. ; Aliaga-Rossel, R. ; BeNali, O. ; Keles, H. ; Lebert, B. ; Sarroukh, O. ; Tantart, L. ; Zaepffel, C. ; Michette, A. ; McNulty, I. ; Eyberger, C. ; LAI, B.: The Development of a Tabletop Soft X-ray in vitroMicroscope. In: AIP Conference Proceedings 1365 (2011), September, Nr. 1. http://dx.doi.org/10.1063/1.3625321. - DOI $10.1063 / 1.3625321$

[76] Nawaz, M. F. ; Nevrkla, M. ; Jancarek, A. ; Torrisi, A. ; Parkman, T. ; Turnova, J. ; Stolcova, L. ; Vrbova, M. ; LimPOUCH, J. ; Pina, L. ; WachulaK, P.: Table-top water-window soft X-ray microscope using a Z-pinching capillary discharge source. In: Journal of Instrumentation 11 (2016), Juli, Nr. 07, S. Po7002Po7002. http://dx.doi.org/10.1088/1748-0221/11/07/P07002. DOI 10.1088/1748-0221/11/07/Po7002

[77] Horne, S. F. ; Besen, M. M. ; Smith, D. K. ; Blackborow, P. A. ; D'Agostino, R.: Application of a high-brightness electrodeless Z-pinch EUV source for metrology, inspection, and resist development. In: SPIE Proceedings 6151 (2006), März, Nr. EUV Source I. http://dx.doi.org/10.1117/12.655696. - DOI $10.1117 / 12.655696$

[78] Mayer-Kuckuк, T.: Atomphysik: Eine Einführung (Teubner Studienbücher Physik). Vieweg+Teubner Verlag, 1997 (5. Auflage). - ISBN 3519430428

[79] Gerthsen, C. ; Kneser, H. O. ; Vogel, H.: Physik: Ein Lehrbuch zum Gebrauch neben Vorlesungen. Springer, 1977 (13. Auflage). ISBN 3540078762

[8o] Fiedorowicz, H. ; Bartnik, A. ; Szczurek, M. ; Daido, H. ; Sakaya, N. ; KMetik, V. ; Kato, Y. ; Suzuki, M. ; Matsumura, M. ; TAjima, J. ; NAKAyama, T. ; Wilhein, T.: Investigation of soft X-ray emission from a gas puff target irradiated with a Nd:YAG laser. In: Optics Communications 163 (1999), Mai, Nr. 1-3, S. 103114. http://dx. doi . org/10 . 1016/S0030-4018(99)00100-5. - DOI 10.1016/Sо030-4018(99)oo100-5

[81] Müller, M. ; Kühl, F.-C. ; Grossmann, P. ; Vrba, P. ; Mann, K.: Emission properties of $\mathrm{ns}$ and ps laser-induced soft $\mathrm{x}$-ray sources using pulsed gas jets. In: Optics Express 21 (2013), Mai, Nr. 
10, S. 12831. http://dx.doi.org/10.1364/0E.21.012831. - DOI 10.1364/OE.21.012831

[82] MacFarlane, J. J. ; Rettig, C. L. ; Wang, P. ; Golovkin, I. E. ; Woodruff, P. R.: Radiation-hydrodynamics, spectral, and atomic physics modeling of laser-produced plasma EUVL light sources. In: SPIE Proceedings 5751 (2005), Mai. http://dx.doi.org/10. 1117/12.600295. - DOI 10.1117/12.600295

[83] MacFarlane, J. J. ; Golovkin, I. E. ; Wang, P. ; Woodruff, P. R. ; Pereyra, N. A.: SPECT3D - A multi-dimensional collisionalradiative code for generating diagnostic signatures based on hydrodynamics and PIC simulation output. In: High Energy Density Physics 3 (2007), Mai, Nr. 1-2, S. 181-190. http://dx.doi.org/10. 1016/j . hedp.2007.02.016. - DOI 10.1016/j.hedp.2007.02.016

[84] Barkusky, F. ; Bayer, A. ; Peth, C. ; Mann, K.: Direct photoetching of polymers using radiation of high energy density from a table-top extreme ultraviolet plasma source. In: Journal of Applied Physics 105 (2009), Januar, Nr. 1, S. 014906. http://dx.doi.org/ 10.1063/1.3054565. - DOI 10.1063/1.3054565

[85] Mantouvalou, I. ; K. ; Grötzsch, D. ; Neitzel, M. ; GünTher, S. ; Baumann, J. ; Jung, R. ; Stiel, H. ; Kanngiesser, B. ; SAndner, W.: High average power, highly brilliant laserproduced plasma source for soft X-ray spectroscopy. In: Review of Scientific Instruments 86 (2015), März, Nr. 3, S. 035116. http: //dx.doi.org/10.1063/1.4916193. - DOI 10.1063/1.4916193

[86] Haney, S. J. ; Berger, K. W. ; Kubiak, G. D. ; Rockett, P. D. ; Hunter, J.: Prototype high-speed tape target transport for a laser plasma soft-x-ray projection lithography source. In: Applied Optics 32 (1993), Dezember, Nr. 34, S. 6934. http: //dx. doi . org/10.1364/ A0.32.006934. - DOI 10.1364/AO.32.006934

[87] Mantouvalou, I. ; Jung, R. ; Tuemmler, J. ; Legall, H. ; BiDU, T. ; Stiel, H. ; Malzer, W. ; Kanngiesser, B. ; SAndner, W.: Note: Study of extreme ultraviolet and soft $x$-ray emission of metal targets produced by laser-plasma-interaction. In: Review of Scientific Instruments 82 (2011), Juni, Nr. 6, S. o66103. http: //dx.doi.org/10.1063/1.3600069. - DOI 10.1063/1.3600069

[88] Ohashi, H. ; Higashiguchi, T. ; Suzuki, Y. ; Arai, G. ; Otani, Y. ; Yatagai, T. ; Li, B. ; Dunne, P. ; O'Sullivan, G. ; Jiang, W. ; Endo, A. ; Sakaue, H. A. ; Kato, D. ; Murakami, I. ; Tamura, N. ; Sudo, S. ; Koike, F. ; Suzuki, C.: Quasi-Moseley's law for strong narrow bandwidth soft $\mathrm{x}$-ray sources containing 
higher charge-state ions. In: Applied Physics Letters 104 (2014), Juni, Nr. 23, S. 234107. http://dx.doi.org/10.1063/1.4883475. - DOI $10.1063 / 1.4883475$

[89] Lebert, R. ; Schriever, G. ; Wilhein, T. ; Niemann, B.: Soft Xray emission of laser-produced plasmas using a low-debris cryogenic nitrogen target. In: Journal of Applied Physics 84 (1998), September, Nr. 6, S. 3419-3421. http://dx. doi .org/10.1063/1.368502. DOI 10.1063/1.368502

[9o] Peth, C. ; Kalinin, A. ; Barkusky, F. ; Mann, K. ; Toennies, J. P. ; RUsIN, L. Y.: XUV laser-plasma source based on solid Ar filament. In: Review of Scientific Instruments 78 (2007), Oktober, Nr. 10, S. 103509. http://dx.doi.org/10.1063/1.2801882. - DOI $10.1063 / 1.2801882$

[91] Berglund, M. ; Rymell, L. ; Hertz, H. M. ; Wilhein, T.: Cryogenic liquid-jet target for debris-free laser-plasma soft x-ray generation. In: Review of Scientific Instruments 69 (1998), Juni, Nr. 6, S. 2361-2364. http://dx.doi.org/10.1063/1.1148944. - DOI $10.1063 / 1.1148944$

[92] Rymell, L. ; Hertz, H.M.: Droplet target for low-debris laserplasma soft X-ray generation. In: Optics Communications 103 (1993), November, Nr. 1-2, S. 105-110. http://dx.doi.org/10.1016/ 0030-4018 (93) 90651-K. - DOI 10.1016/0030-4018(93)90651-K

[93] Vogt, U. ; Frueke, R. ; Wilhein, T. ; Stollberg, H. ; Jansson, P. A. C. ; Hertz, H. M.: High-resolution spatial characterization of laser produced plasmas at soft x-ray wavelengths. In: Applied Physics B 78 (2004), Januar, Nr. 1, S. 53-58

[94] Komori, H. ; Ueno, Y. ; Hoshino, H. ; Ariga, T. ; SoumaGNe, G. ; ENdo, A. ; Mizoguchi, H.: EUV radiation characteristics of a $\mathrm{CO}_{2}$ laser produced Xe plasma. In: Applied Physics $B$ 83 (2006), März, Nr. 2, S. 213-218. http://dx.doi.org/10.1007/ s00340-006-2172 - 7. - DOI 10.1007/s00340-006-2172-7

[95] Wieland, M. ; Wilhein, T. ; Faubel, M. ; Ellert, C. ; Schmidt, M. ; Sublemontier, O.: EUV and fast ion emission from cryogenic liquid jet target laser-generated plasma. In: Applied Physics $B$ 72 (2001), April, Nr. 5, S. 591-597. http://dx.doi.org/10.1007/ s003400100542. - DOI 10.1007/s003400100542

[96] Letardi, T. ; Lo, D. ; Zheng, C.-E.: Particle dynamics of debris produced during laser-plasma soft x-ray generation. In: Journal of Applied Physics 89 (2001), Januar, Nr. 2, S. 1458-1462. http://dx. doi.org/10.1063/1.1334365. - DOI 10.1063/1.1334365 
[97] Jin, F. ; Richardson, M.: New laser plasma source for extremeultraviolet lithography. In: Applied Optics 34 (1995), September, Nr. 25, S. 5750-5760. http://dx. doi.org/10.1364/A0.34.005750. - DOI 10.1364/AO.34.005750

[98] Elg, D. T. ; Sporre, J. R. ; Curreli, D. ; Shchelkanov, I. A. ; Ruzic, D. N. ; Umstadter, K. R.: Magnetic debris mitigation system for extreme ultraviolet sources. In: Journal of Micro/Nanolithography, MEMS, and MOEMS 14 (2015), Februar, Nr. 1, S. 013506. http://dx.doi.org/10.1117/1.JMM.14.1.013506. - DOI 10.1117/1.JMM.14.1.013506

[99] Ginter, M. L. ; McIlrath, T. J.: Debris and VUV emission from a laser-produced plasma operated at $150 \mathrm{~Hz}$ using a krypton fluoride laser. In: Applied Optics 27 (1988), März, Nr. 5, S. 885-889. http://dx.doi.org/10.1364/A0.27.000885. - DOI $10.1364 /$ AO.27.000885

[10o] Rymell, L. ; Hertz, H. M.: Debris elimination in a droplet-target laser-plasma soft x-ray source. In: Review of Scientific Instruments 66 (1995), Oktober, Nr. 10, S. 4916-4920. http://dx.doi.org/10. 1063/1.1146174. - DOI 10.1063/1.1146174

[101] BAKshi, V. (Hrsg.): EUV Lithography. JOHN WILEY \& SONS INC, 2009. - ISBN 9780470471555

[102] Semushin, S. ; Malka, V.: High density gas jet nozzle design for laser target production. In: Review of Scientific Instruments 72 (2001), Juli, Nr. 7, S. 2961-2965. http: //dx. doi . org/10.1063/1. 1380393. - DOI 10.1063/1.1380393

[103] Peth, C. ; Kranzusch, S. ; Mann, K. ; Viöl, W.: Characterization of gas targets for laser produced extreme ultraviolet plasmas with a Hartmann-Shack sensor. In: Review of Scientific Instruments 75 (2004), Oktober, Nr. 10, S. 3288-3293. http://dx.doi.org/10. 1063/1.1791314. - DOI 10.1063/1.1791314

[104] Fiedorowicz, H. ; Bartnik, A. ; Daido, H. ; Choi, I. W. ; SuzuKI, M. ; YAMAGAMI, S.: Strong extreme ultraviolet emission from a double-stream xenon/helium gas puff target irradiated with a Nd:YAG laser. In: Optics Communications 184 (2000), Oktober, Nr. 1-4, S. 161-167. http://dx. doi . org/10 . 1016/S0030-4018 (00) 00946 - 9. - DOI 10.1016/Soo30-4018(oo)00946-9

[105] Mey, T. ; Rein, M. ; Grossmann, P. ; Mann, K.: Brilliance improvement of laser-produced soft x-ray plasma by a barrel shock. In: New Journal of Physics 14 (2012), Juli, Nr. 7, S. 
073045. http://dx.doi.org/10.1088/1367-2630/14/7/073045. DOI 10.1088/1367-2630/14/7/073045

[106] Proch, D. ; TrickL, T.: A high-intensity multi-purpose piezoelectric pulsed molecular beam source. In: Review of Scientific Instruments 6o (1989), April, Nr. 4, S. 713-716. http://dx.doi.org/10. 1063/1.1141006. - DOI 10.1063/1.1141006

[107] Colombant, D. ; Tonon, G. F.: X-ray emission in laser-produced plasmas. In: Journal of Applied Physics 44 (1973), August, Nr. 8, S. 3524-3537. http://dx.doi.org/10.1063/1.1662796. - DOI $10.1063 / 1.1662796$

[108] Li, Y. M. ; Fedosejevs, R.: Density measurements of a highdensity pulsed gas jet for laser-plasma interaction studies. In: Measurement Science and Technology 5 (1994), Oktober, Nr. 10, S. 11971201. http://dx.doi.org/10.1088/0957-0233/5/10/002. - DOI 10.1088/0957-0233/5/10/002

[109] Fiedorowicz, H. ; Bartnik, A. ; Szczurek, M. ; Daido, H. ; Sakaya, N. ; KMetik, V. ; Kato, Y. ; Suzuki, M. ; Matsumura, M. ; TAjima, J. ; NAKAyama, T. ; Wilhein, T.: Investigation of soft X-ray emission from a gas puff target irradiated with a Nd:YAG laser. In: Optics Communications 163 (1999), Mai, Nr. 1-3, S. 103114. http://dx.doi.org/10.1016/S0030-4018(99)00100-5. - DOI 10.1016/So030-4018(99)о0100-5

[110] Li, B. ; Higashiguchi, T. ; Otsuka, T. ; Jiang, W. ; Endo, A. ; Dunne, P. ; O'Sullivan, G.: Water window sources: Selection based on the interplay of spectral properties and multilayer reflection bandwidth. In: Applied Physics Letters 102 (2013), Januar, Nr. 4, S. 041117. http://dx.doi.org/10.1063/1.4789982. - DOI $10.1063 / 1.4789982$

[111] Hagena, O. F.: Nucleation and growth of clusters in expanding nozzle flows. In: Surface Science 106 (1981), Mai, Nr. 1-3, S. 101116. http://dx.doi.org/10.1016/0039-6028(81)90187-4. - DOI 10.1016/0039-6028(81)90187-4

[112] Ditmire, T. ; Donnelly, T. ; Rubenchik, A. M. ; Falcone, R. W. ; Perry, M. D.: Interaction of intense laser pulses with atomic clusters. In: Physical Review A 53 (1996), Mai, Nr. 5, S. 33793402. http://dx.doi.org/10.1103/PhysRevA.53.3379. - DOI 10.1103/PhysRevA.53.3379

[113] Holburg, J.: Charakterisierung einer Hochdruckdüse zur Erzeugung weicher Röntgenstrahlung mittels laserinduzierter Plasmen, Hochschule für angewandte Wissenschaft und Kunst in Göttingen 
und Laser-Laboratorium Göttingen e.V., Bachelorarbeit, September 2015

[114] Kanouff, M. ; Shields, H. ; Bernardez, L. ; Chenoweth, D. ; KUBIAK, G.: Absorption of extreme ultraviolet light in a laser produced gas-jet plasma source. In: Journal of Applied Physics 90 (2001), Oktober, Nr. 8, S. 3726-3734. http://dx. doi . org/10.1063/ 1.1403681. - DOI 10.1063/1.1403681

[115] Kranzusch, S. ; Peth, C. ; Mann, K.: Spatial characterization of extreme ultraviolet plasmas generated by laser excitation of xenon gas targets. In: Review of Scientific Instruments 74 (2003), Februar, Nr. 2, S. 969-974. http://dx.doi.org/10.1063/1.1532835. - DOI $10.1063 / 1.1532835$

[116] Settles, G. S.: Schlieren and Shadowgraph Techniques. Springer Berlin Heidelberg, 2012. - ISBN 978-3-642-63034-7

[117] Wolter, H.: Spiegelsysteme streifenden Einfalls als abbildende Optiken für Röntgenstrahlen. In: Annalen der Physik 445 (1952), Nr. 1-2, S. 94-114. http://dx.doi.org/10.1002/andp. 19524450108. DOI 10.1002/andp.19524450108

[118] Kirz, J. ; JAcobsen, C.: The history and future of X-ray microscopy. In: Journal of Physics: Conference Series 186 (2009), September, S. 012001. http://dx.doi.org/10.1088/1742-6596/186/1/012001. DOI 10.1088/1742-6596/186/1/012001

[119] Howells, M. ; Jacobsen, C. ; Warwick, T. ; Bos, A.: Principles and Applications of Zone Plate X-Ray Microscopes. Version: 2007. http://dx.doi.org/10.1007/978-0-387-49762-4_13. In: Science of Microscopy. Springer New York, 2007. - DOI 10.1007/978-0387-49762-4_13, S. 835-926

[120] Henke, B. ; Gullikson, E. ; Davis, J.: X-ray interactions: photoabsorption, scattering, transmission, and reflection at $\mathrm{E}=5 \mathrm{O}-30000$ $\mathrm{eV}, \mathrm{Z}=1-92$. In: Atomic Data and Nuclear Data Tables 54 (1993), Juli, Nr. 2, S. 191-342. http: //dx. doi . org/10. 1006/adnd. 1993. 1013. DOI 10.1006/adnd.1993.1013

[121] Michelson, A.: Studies in Optics. Dover Publications, 1995. ISBN 0-486-68700-7

[122] Rayleigh, J.: XXXI. Investigations in optics, with special reference to the spectroscope. In: Philosophical Magazine Series 58 (1879), Oktober, Nr. 49, S. 261-274. http://dx. doi.org/10.1080/ 14786447908639684. - DOI 10.1080/14786447908639684 
[123] Chao, W. ; Fischer, P. ; Tyliszczak, T. ; Rekawa, S. ; AnDerson, E. ; Naulleau, P.: Real space soft x-ray imaging at $10 \mathrm{~nm}$ spatial resolution. In: Optics Express 20 (2012), April, Nr. 9, S. 9777. http://dx.doi.org/10.1364/0E.20.009777. - DOI 10.1364/OE.20.009777

[124] Williams, C. ; Becklund, O.: Introduction to the Optical Transfer Function. Bd. PM 112. SPIE Publications, 2002. - ISBN 9780819443366

[125] Adam, J.-F. ; Moy, J.-P. ; Susini, J.: Table-top water window transmission x-ray microscopy: Review of the key issues, and conceptual design of an instrument for biology. In: Review of Scientific Instruments 76 (2005), September, Nr. 9, S. o91301. http: //dx.doi.org/10.1063/1.2018633. - DOI 10.1063/1.2018633

[126] Snigirev, A. ; Kohn, V. ; Snigireva, I. ; Lengeler, B.: A compound refractive lens for focusing high-energy X-rays. In: Nature 384 (1996), November, Nr. 6604, S. 49-51. http://dx. doi.org/10. 1038/384049a0. - DOI 10.1038/384049ao

[127] Schroer, C. G. ; Kurapova, O. ; Patommel, J. ; Boye, P. ; FeldKAMP, J. ; Lengeler, B. ; Burghammer, M. ; Riekel, C. ; Vincze, L. ; HaRt, A. van d. ; Küchler, M.: Hard x-ray nanoprobe based on refractive x-ray lenses. In: Applied Physics Letters 87 (2005), September, Nr. 12, S. 124103. http: //dx. doi . org/10 . 1063/1.2053350. - DOI 10.1063/1.2053350

[128] Michette, A.: Optical Systems for Soft X Rays. Springer, 2011. ISBN 9781461293040

[129] Yulin, S. ; Benoit, N. ; Feigl, T. ; Kaiser, N.: Interfaceengineered EUV multilayer mirrors. In: Microelectronic Engineering 83 (2006), April, Nr. 4-9, S. 692-694. http://dx. doi .org/10.1016/ j.mee.2006.01.126. - DOI 10.1016/j.mee.2006.01.126

[130] BAKSHI, V.: EUV Lithography. Wiley, 2008. - ISBN 9780470471555

[131] UnderWOOd, J. ; BARBEE, T.: Layered synthetic microstructures as Bragg diffractors for $X$ rays and extreme ultraviolet: theory and predicted performance. In: Applied Optics 20 (1981), September, Nr. 17, S. 3027. http://dx.doi.org/10.1364/A0.20.003027. - DOI 10.1364/AO.20.003027

[132] Gullikson, E. ; Salmassi, F. ; Aquila, A. ; Dollar, F.: Progress in short period multilayer coatings for water window applications. 8th 
International Conference on the Physics of X-Ray Multilayer Structures, Japan. www. osti.gov/scitech/biblio/932470. Version: Mai 2006

[133] Stollberg, H. ; Yulin, S. ; Takman, P. ; Hertz, H.: Highreflectivity $\mathrm{Cr} / \mathrm{Sc}$ multilayer condenser for compact soft x-ray microscopy. In: Review of Scientific Instruments 77 (2006), Dezember, Nr. 12, S. 123101. http://dx.doi.org/10.1063/1.2400665. - DOI $10.1063 / 1.2400665$

[134] Fogelqvist, E. ; Kördel, M. ; Carannante, V. ; Önfelt, B. ; HeRtz, H.: Laboratory cryo x-ray microscopy for $3 \mathrm{D}$ cell imaging. In: Scientific Reports 7 (2017), Oktober, Nr. 1. http://dx. doi.org/ 10.1038/s41598-017-13538-2. - DOI 10.1038/s41598-017-135382

[135] Yulin, S. ; Feigl, T. ; Benoit, N. ; Perske, M. ; Taracheva, E. ; Schröder, S. ; Nesterenko, V. ; Kaiser, N.: Hochreflektierende EUV/Röntgen-Multilayerspiegel. Jahresbericht, 2007. - Fraunhofer IOF Jena

[136] Compton, A.: The total reflexion of X-rays. In: The London, Edinburgh, and Dublin Philosophical Magazine and Journal of Science 45 (1923), Juni, Nr. 270, S. 1121-1131. http://dx.doi.org/10.1080/ 14786442308634208. - DOI 10.1080/14786442308634208

[137] Arndt, U. W. ; Duncumb, P. ; Long, J. V. P. ; Pina, L. ; InneMAN, A.: Focusing Mirrors for Use with Microfocus X-ray Tubes. In: Journal of Applied Crystallography 31 (1998), Oktober, Nr. 5, S. 733-741. http://dx.doi.org/10.1107/S0021889898004439. - DOI 10.1107/Soo21889898004439

[138] Kirkpatrick, P. ; Baez, A.: Formation of Optical Images by XRays. In: Journal of the Optical Society of America 38 (1948), September, Nr. 9, S. 766. http: //dx. doi . org/10. 1364/J0SA. 38.000766. DOI 10.1364/JOSA.38.000766

[139] Montel, M.: X-ray microscopy with catamegonic roof mirrors. In: X-ray Microscopy and Microradiography (1957), S. 177-185

[140] Hudec, R. ; PinA, L. ; Inneman, A.: Replicated grazing incidence x-ray optics: past, present, and future. In: Hoover, R. (Hrsg.) ; Walker, A. (Hrsg.): X-Ray Optics, Instruments, and Missions II, SPIE, September 1999, S. 3766

[141] KIRz, J.: Phase zone plates for $x$ rays and the extreme uv. In: Journal of the Optical Society of America 64 (1974), März, Nr. 3, S. 301. 
http://dx.doi.org/10.1364/J0SA.64.000301. - DOI 10.1364/JOSA.64.000301

[142] Schmahl, G. ; Rudolph, D. ; Schneider, G. ; Thieme, J. ; Schliebe, T. ; Kaulich, B. ; Hettwer, M.: Diffraction optics for X-ray imaging. In: Microelectronic Engineering 32 (1996), September, Nr. 1-4, S. 351-367. http://dx.doi.org/10.1016/0167-9317(95) 00177- 8. - DOI 10.1016/0167-9317(95)00177-8

[143] Holmberg, A. ; Rehbein, S. ; Hertz, H.: Nano-fabrication of condenser and micro-zone plates for compact X-ray microscopy. In: Microelectronic Engineering 73-74 (2004), Juni, S. 639643. http://dx.doi.org/10.1016/j.mee.2004.03.006. - DOI 10.1016/j.mee.2004.03.006

[144] Werner, S. ; Rehbein, S. ; Guttman, P. ; Heim, S. ; SchneiDER, G.: Towards stacked zone plates. In: Journal of Physics: Conference Series 186 (2009), Nr. 1, S. 012079. http://dx. doi . org/10 . 1088/1742 -6596/186/1/012079. - DOI 10.1088/17426596/186/1/012079

[145] Shastri, S. ; Maser, J. ; LaI, B. ; Tys, J.: Microfocusing of 50 $\mathrm{keV}$ undulator radiation with two stacked zone plates. In: Optics Communications 197 (2001), September, Nr. 1-3, S. 9-14. http://dx. doi . org/10 . 1016/S0030-4018(01) 01408- 0. - DOI 10.1016/Soo3o4018(о1)01408-0

[146] Feng, Y. ; Feser, M. ; Lyon, A. ; Rishton, S. ; Zeng, X. ; Chen, S. ; SAssolini, S. ; YUN, W.: Nanofabrication of high aspect ratio $24 \mathrm{~nm} x$-ray zone plates for x-ray imaging applications. In: Journal of Vacuum Science \& Technology B, Nanotechnology and Microelectronics: Materials, Processing, Measurement, and Phenomena 25 (2007), Nr. 6, S. 2004. http://dx.doi.org/10.1116/1.2789447. - DOI $10.1116 / 1.2789447$

[147] Mohacsi, I. ; Karvinen, P. ; Vartiainen, I. ; Guzenko, V. ; Somogyi, A. ; Kewish, C. ; Mercere, P. ; David, C.: Highefficiency zone-plate optics for multi-keV X-ray focusing. In: Journal of Synchrotron Radiation 21 (2014), März, Nr. 3, S. 497501. http://dx.doi.org/10.1107/S1600577514003403. - DOI 10.1107/S1600577514003403

[148] Jefimovs, K. ; Vila-Comamala, J. ; Pilvi, T. ; RaAbe, J. ; Ritala, M. ; David, C.: Zone-Doubling Technique to Produce Ultrahigh-Resolution X-Ray Optics. In: Physical Review Letters 99 (2007), Dezember, Nr. 26. http://dx.doi.org/10.1103/ PhysRevLett. 99.264801. - DOI 10.1103/PhysRevLett.99.264801 
[149] Vila-Comamala, J. ; Gorelick, S. ; Färm, E. ; Kewish, C. ; Diaz, A. ; Guzenko, V. ; Barrett, R. ; RaAbe, J. ; Menzel, A. ; Bunk, O. ; Ritala, M. ; David, C. ; McNulty, I. ; Eyberger, C. ; LAI, B.: Zone-Doubled Fresnel Zone Plates for Scanning Transmission X-ray Microscopy. In: AIP Conference Proceedings 1365 (2011), Nr. 1, S. 192-195. http://dx.doi.org/10.1063/1.3625337. - DOI $10.1063 / 1.3625337$

[150] Lauterborn, W. ; Kurz, T.: Coherent Optics: Fundamentals and Applications. Springer, 2003. - ISBN 3-540-43933-1

[151] Born, M. ; Wolf, E.: Principles of Optics: Electromagnetic Theory of Propagation, Interference and Diffraction of Light. Cambridge University Press, 1999. - ISBN 978-0521642224

[152] Demtröder, W.: Experimentalphysik 2: Elektrizität und Optik. Springer, 2014. - ISBN 978-3-642-29944-5

[153] Michelson, A. ; Morley, E.: On the relative motion of the earth and the luminiferous Ether. In: The London, Edinburgh, and Dublin Philosophical Magazine and Journal of Science 24 (1887), Dezember, Nr. 151, S. 449-463. http://dx.doi.org/10.1080/ 14786448708628130. - DOI 10.1080/14786448708628130

[154] Young, T.: The Bakerian Lecture: Experiments and Calculations Relative to Physical Optics. In: Philosophical Transactions of the Royal Society of London 94 (1804), Januar, Nr. O, S. 1-16. http://dx. doi. org/10.1098/rstl. 1804.0001. - DOI 10.1098/rstl.1804.0001

[155] Rudolph, D. ; Niemann, B. ; Schmahl, G. ; Christ, O.: The Göttingen X-Ray Microscope and X-Ray Microscopy Experiments at the BESSY Storage Ring. Version: 1984. http://dx.doi.org/ 10. 1007/978-3-540-38833-3_21. In: SchмaнL, G. (Hrsg.) ; RuDOLPH, D. (Hrsg.): X-Ray Microscopy. Springer Berlin Heidelberg, 1984. - DOI 10.1007/978-3-540-38833-3_21, S. 192-202

[156] Kim, K. ; Kwon, Y. ; NAM, K.-Y. ; LIM, J.-H. ; KIM, K.-G. ; CHON, K. ; KIm, B. ; KIM, D. ; KIM, J. ; Ahn, B. ; Shin, H. ; RAH, S. ; KIM, K.-H. ; Chae, J. ; Gweon, D. ; Kang, D. ; Kang, S. ; Min, J. ; ChoI, K.-S. ; Yoon, S. ; Kim, E. ; NAmbA, Y. ; Yoon, K.-H.: Compact soft $\mathrm{x}$-ray transmission microscopy with sub-50 $\mathrm{nm}$ spatial resolution. In: Physics in Medicine and Biology 51 (2006), Februar, Nr. 6, S. N99Nio7. http://dx.doi.org/10.1088/0031-9155/51/6/N01. - DOI 10.1088/0031-9155/51/6/No1

[157] Jochum, L. ; Meyer-Ilse, W.: Partially coherent image formation with x-ray microscopes. In: Applied Optics 34 (1995), August, Nr. 
22, S. 4944. http://dx.doi.org/10.1364/A0.34.004944. - DOI 10.1364/AO.34.004944

[158] Schmahl, G. ; Rudolph, D. ; Guttmann, P.: Phase Contrast X-Ray Microscopy - Experiments at the BESSY Storage Ring. In: SAyre, D. (Hrsg.) ; Kirz, J. (Hrsg.) ; Howells, M. (Hrsg.) ; RaRBACK, H. (Hrsg.): X-Ray Microscopy II, Springer Berlin Heidelberg, 1988, S. 228-232

[159] Denbeaux, G. ; Fischer, P. ; Salmassi, F. ; Dunn, K. ; EvertSEN, J.: Reflection Mode Imaging with High Resolution X-ray Microscopy. Conference: The 8th International Conference on XrayMicroscopy, Egret Himeji, Japan, Juli 2005

[160] Brizuela, F. ; Vaschenko, G. ; Brewer, C. ; Grisham, M. ; Menoni, C. ; Marconi, M. ; Rocca, J. ; Chao, W. ; LiddLe, J. ; Anderson, E. ; Attwood, D. ; Vinogradov, A. ; Artioukov, I. ; Pershyn, Y. ; Kondratenko, V.: Reflection mode imaging with nanoscale resolution using a compact extreme ultraviolet laser. In: Optics Express 13 (2005), Nr. 11, S. 3983. http://dx.doi.org/10.1364/OPEX.13.003983. - DOI 10.1364/OPEX.13.003983

[161] Schmahl, G. (Hrsg.) ; Rudolph, D. (Hrsg.): X-Ray Microscopy. Springer Berlin Heidelberg, 1984. http://dx.doi. org/10 . 1007/978-3-540-38833-3. http://dx. doi .org/10.1007/ $978-3-540-38833-3$

[162] Sayre, D. ; Howells, M. ; KIrz, J.: X-Ray Microscopy II. Springer Verlag, 1988. - ISBN 978-0387193922

[163] Michette, A. (Hrsg.) ; Morrison, G. (Hrsg.) ; Buckley, C. (Hrsg.): X-Ray Microscopy III. Springer Berlin Heidelberg, 1992. http://dx.doi.org/10.1007/978-3-540-46887-5. http: //dx.doi.org/10.1007/978-3-540-46887-5

[164] Aristov, V. (Hrsg.) ; Erko, A. (Hrsg.): X-ray Microscopy IV. Chernogolovka, Russia: Bogorodskii Pechatnik, 1993. - ISBN 5-90084601-6

[165] Thieme, J. (Hrsg.) ; Schmahl, G. (Hrsg.) ; Rudolph, D. (Hrsg.) ; Uмвасн, E. (Hrsg.): X-Ray Microscopy and Spectromicroscopy. Springer Berlin Heidelberg, 1998. http://dx.doi. org/10.1007/978-3-642-72106-9. http://dx. doi . org/10.1007/ $978-3-642-72106-9$

[166] Meyer-Ilse, M. (Hrsg.) ; Warwick, T. (Hrsg.) ; Attwood, D. (Hrsg.): X-Ray Microscopy: Proceedings of the Sixth International Conference. American Institute of Physics, 2000. - ISBN 1-56396-926-2 
[167] Susini, J. ; Joyeux, D. ; Polack, F.: $7^{\text {th International Conference }}$ on X-Ray Microscopy. In: Journal de Physique 104 (2003), März, S. V

[168] Aoki, S. (Hrsg.) ; Kagoshima, Y. (Hrsg.) ; Suzuki, Y. (Hrsg.): 8th International Conference on X-ray Microscopy. IPAP, 2006

[169] Quitmann, C. ; David, C. ; Nolting, F. ; Pfeiffer, F. ; StamPANONI, M.: 9th International Conference on X-Ray Microscopy. In: Journal of Physics: Conference Series 186 (2009), September, S. o11001. http://dx.doi.org/10.1088/1742-6596/186/1/011001. DOI 10.1088/1742-6596/186/1/011001

[170] McNulty, I. (Hrsg.) ; Eyberger, C. (Hrsg.) ; LaI, B. (Hrsg.): The 1oth International Conference on X-Ray Microscopy. Bd. 1365. American Institute of Physics, 2011. - ISBN 978-0-7354-0925-5

[171] XU, H. ; WU, Z. ; TAI, R.: 11th International Conference on XRay Microscopy. In: Journal of Physics: Conference Series 463 (2013), Oktober, S. O11001. http://dx.doi .org/10 . 1088/1742-6596/463/ 1/011001. - DOI 10.1088/1742-6596/463/1/011001

[172] Jonge, M. de ; Paterson, D. ; Ryan, C.: Preface: The International Conference on X-Ray Microscopy. In: AIP Conference Proceedings 1696 (2016), Januar, S. 010001. http: //dx. doi.org/10. 1063/ 1.4937493. - DOI 10.1063/1.4937493

[173] Rau, C.: X-Ray Microscopy Conference 2016. In: Journal of Physics: Conference Series 849 (2017), Juni, S. o11001. http://dx. doi .org/10.1088/1742-6596/849/1/011001. - DOI 10.1088/17426596/849/1/011001

[174] Marchesini, S. ; Chapman, H. ; Hau-Riege, S. ; London, R. ; Szoke, A. ; He, H. ; Howells, M. ; Padmore, H. ; Rosen, R. ; Spence, J. ; Weierstall, U.: Coherent X-ray diffractive imaging: applications and limitations. In: Optics Express 11 (2003), September, Nr. 19, S. 2344. http : //dx. doi . org/10. 1364/0E . 11.002344. DOI 10.1364/OE.11.002344

[175] Kapitel D. Sayre - Prospects for long-wavelength x-ray microscopy and diffraction. In:Schlenker, M. ; Fink, M. ; Goedgebuer, J. ; Malgrange, C. ; Viénot, J. ; WAdE, R.: Imaging processes and coherence in physics. Bd. 112. Springer-Verlag Berlin, Heidelberg, New York, 1980, S. 229-235

[176] Sayre, D. ; Chapman, H. ; Miao, J.: On the Extendibility of X-ray Crystallography to Noncrystals. In: Acta Crystallographica Section A Foundations of Crystallography 54 (1998), März, Nr. 2, S. 
232-239. http://dx.doi.org/10.1107/S0108767397015572. - DOI 10.1107/So108767397015572

[177] Miao, J. ; Charalambous, P. ; Kirz, J. ; Sayre, D.: Extending the methodology of X-ray crystallography to allow imaging of micrometre-sized non-crystalline specimens. In: Nature 400 (1999), Juli, Nr. 6742, S. 342-344. http://dx.doi.org/10.1038/22498. DOI 10.1038/22498

[178] Miao, J. ; Ishikawa, T. ; Shen, Q. ; Earnest, T.: Extending XRay Crystallography to Allow the Imaging of Noncrystalline Materials, Cells, and Single Protein Complexes. In: Annual Review of Physical Chemistry 59 (2008), Mai, Nr. 1, S. 387-410. http://dx. doi.org/10.1146/annurev. physchem.59.032607.093642. - DOI 10.1146/annurev.physchem.59.032607.093642

[179] Pfeiffer, F.: X-ray ptychography. In: Nature Photonics 12 (2017), Dezember, Nr. 1, S. 9-17. http://dx.doi.org/10.1038/ s41566- 017 - 0072 - 5. - DOI 10.1038/s41566-017-0072-5

[180] Hoppe, W.: Beugung im inhomogenen Primärstrahlwellenfeld. III. Amplituden- und Phasenbestimmung bei unperiodischen Objekten. In: Acta Crystallographica Section A 25 (1969), Juli, Nr. 4, S. 508-514. http://dx.doi.org/ https://doi.org/10.1107/S0567739469001069. - DOI https://doi.org/10.1107/So567739469001069

[181] Rodenburg, J. ; Hurst, A. ; Cullis, A. ; Dobson, B. ; Pfeiffer, F. ; Bunk, O. ; DAvid, C. ; Jefimovs, K. ; Johnson, I.: Hard-X-Ray Lensless Imaging of Extended Objects. In: Physical Review Letters 98 (2007), Januar, Nr. 3. http://dx.doi.org/10.1103/PhysRevLett. 98.034801. - DOI 10.1103/PhysRevLett.98.034801

[182] Thibault, P. ; Dierolf, M. ; Menzel, A. ; Bunk, O. ; David, C. ; Pfeiffer, F.: High-Resolution Scanning X-ray Diffraction Microscopy. In: Science 321 (2008), Juli, Nr. 5887, S. 379-382. http://dx.doi.org/10.1126/science.1158573. - DOI 10.1126/science.1158573

[183] Dierolf, M. ; Thibault, P. ; Menzel, A. ; Kewish, C. ; Jefimovs, K. ; Schlichting, I. ; König, K. von ; Bunk, O. ; Pfeiffer, F.: Ptychographic coherent diffractive imaging of weakly scattering specimens. In: New Journal of Physics 12 (2010), März, Nr. 3, S. 035017. http://dx.doi.org/10.1088/1367-2630/12/3/035017. - DOI 10.1088/1367-2630/12/3/035017 
[184] Dierolf, M. ; Menzel, A. ; Thibault, P. ; Schneider, P. ; Kewish, C. ; Wepf, R. ; Bunk, O. ; Pfeiffer, F.: Ptychographic Xray computed tomography at the nanoscale. In: Nature 467 (2010), September, Nr. 7314, S. 436-439. http://dx.doi.org/10.1038/ nature09419. - DOI 10.1038/natureo9419

[185] Beckers, M. ; Senkbeil, T. ; Gorniak, T. ; Reese, M. ; Giewekemeyer, K. ; Gleber, S.-C. ; Salditt, T. ; Rosenhahn, A.: Chemical Contrast in Soft X-Ray Ptychography. In: Physical Review Letters 107 (2011), November, Nr. 20. http://dx.doi.org/10.1103/ PhysRevLett. 107.208101. - DOI 10.1103/PhysRevLett.107.208101

[186] Shapiro, D. ; Yu, Y.-S. ; Tyliszczak, T. ; Cabana, J. ; Celestre, R. ; Chao, W. ; Kaznatcheev, K. ; Kilcoyne, D. ; Maia, F. ; Marchesini, S. ; Meng, S. ; Warwick, T. ; Yang, L. ; Padmore, H.: Chemical composition mapping with nanometre resolution by soft X-ray microscopy. In: Nature Photonics 8 (2014), September, Nr. 10, S. 765-769. http://dx. doi .org/10.1038/nphoton. 2014.207. DOI 10.1038/nphoton.2014.207

[187] McNulty, I. ; Kirz, J. ; Jacobsen, C. ; Anderson, E. ; Howells, M. ; KERN, D.: High-Resolution Imaging by Fourier Transform X-ray Holography. In: Science 256 (1992), Mai, Nr. 5059, S. 1009-1012. http://dx.doi.org/10.1126/science. 256.5059.1009. - DOI 10.1126/science.256.5059.1009

[188] Eisebitt, S. ; Lüning, J. ; Schlotter, W. ; Lörgen, M. ; HellWIG, O. ; EbERHARdT, W. ; STÖHR, J.: Lensless imaging of magnetic nanostructures by X-ray spectro-holography. In: Nature 432 (2004), Dezember, Nr. 7019, S. 885-888. http://dx.doi.org/10. 1038/nature03139. - DOI 10.1038/natureo3139

[189] Sayre, D. ; Kirz, J. ; Feder, R. ; Kim, D. ; Spiller, E.: Transmission microscopy of unmodified biological materials. Comparative radiation dosages with electrons and ultrasoft $X$-ray photons. In: Ultramicroscopy 2 (1976), Januar, S. 337-349. http://dx. doi . org/10 . 1016/S0304-3991(76)91997-5. - DOI 10.1016/So3043991(76)91997-5

[190] Schneider, G.: Cryo X-ray microscopy with high spatial resolution in amplitude and phase contrast. In: Ultramicroscopy 75 (1998), November, Nr. 2, S. 85-104. http://dx.doi.org/10.1016/ S0304 - 3991 ( 98 ) 00054 - 0. - DOI 10.1016/So304-3991(98)00054-0

[191] Chapman, H. ; Barty, A. ; Bogan, M. ; Boutet, S. ; Frank, M. ; Hau-Riege, S. ; Marchesini, S. ; Woods, B. ; Bajt, S. ; Benner, H. ; London, R. ; Plönjes, E. ; Kuhlmann, M. ; Treusch, R. ; 
Düsterer, S. ; Tschentscher, T. ; Schneider, J. ; Spiller, E. ; Möller, T. ; Bostedt, C. ; Hoener, M. ; Shapiro, D. ; Hodgson, K. ; Spoel, D. van d. ; Burmeister, F. ; Bergh, M. ; Caleman, C. ; Huldt, G. ; Seibert, M. ; Maia, F. ; Lee, R. ; Szöke, A. ; Timneanu, N. ; Hajdu, J.: Femtosecond diffractive imaging with a soft-X-ray free-electron laser. In: Nature Physics 2 (2006), November, Nr. 12, S. 839-843. http://dx.doi.org/10.1038/nphys461. DOI 10.1038/nphys461

[192] Bergh, M. ; Huldt, G. ; Timneanu, N. ; Maia, F. ; Hajdu, J.: Feasibility of imaging living cells at subnanometer resolutions by ultrafast X-ray diffraction. In: Quarterly Reviews of Biophysics 41 (2008), November, Nr. 3-4, S. 181. http://dx.doi.org/10.1017/ S003358350800471X. - DOI 10.1017/So03358350800471X

[193] Nakayama, S. ; Haramura, K. ; Zeng, G.-M. ; Daido, H. ; NaKatsuka, M. ; Nakai, S. ; Katakura, N. ; Nagata, H. ; Ariтоме, H.: Zone-Plate X-Ray Microscope Using a Laser Plasma Source. In: Japanese Journal of Applied Physics 33 (1994), September, Nr. Part 2, No. 9A, S. L128o-L1282. http://dx. doi.org/10.1143/ JJAP. 33. L1280. - DOI 10.1143/JJAP.33.L1280

[194] Rudolph, D. ; Schmahl, G. ; Niemann, B. ; Diehl, M. ; Thieme, J. ; Wilhein, T. ; David, C. ; Michelmann, K.: Wet specimen imaging with an x-ray microscope with a pulsed plasma source. In: X-Ray Microscopy IV (1994), S. 381-386

[195] Takman, P. ; Stollberg, H. ; Johansson, G. ; Holmberg, A. ; Lindblom, M. ; Hertz, H.: High-resolution compact X-ray microscopy. In: Journal of Microscopy 226 (2007), Mai, Nr. 2, S. 175-181. http://dx.doi.org/10.1111/j.1365-2818.2007.01765.x. - DOI $10.1111 /$ j.1365-2818.2007.01765.x

[196] Hofsten, O. von ; Bertilson, M. ; Reinspach, J. ; Holmberg, A. ; Hertz, H. ; Vogt, U.: Sub-25-nm laboratory x-ray microscopy using a compound Fresnel zone plate. In: Optics Letters 34 (2009), August, Nr. 17, S. 2631. http://dx.doi.org/10.1364/0L. 34.002631. - DOI 10.1364/OL.34.002631

[197] Hofsten, O. von ; Bertilson, M. ; Lindblom, M. ; Holmberg, A. ; Vogt, U.: Compact Zernike phase contrast x-ray microscopy using a single-element optic. In: Optics Letters 33 (2008), April, Nr. 9, S. 932. http://dx.doi.org/10.1364/0L.33.000932. - DOI $10.1364 /$ OL.33.000932

[198] Martz, D. ; Selin, M. ; Hofsten, O. von ; Fogelqvist, E. ; Holmberg, A. ; Vogt, U. ; Legall, H. ; Blobel, G. ; Seim, C. 
; Stiel, H. ; Hertz, H.: High average brightness water window source for short-exposure cryomicroscopy. In: Optics Letters 37 (2012), Oktober, Nr. 21, S. 4425. http://dx.doi.org/10.1364/0L. 37.004425. - DOI 10.1364/OL.37.004425

[199] Legall, H. ; Blobel, G. ; Stiel, H. ; Sandner, W. ; Seim, C. ; Takman, P. ; Martz, D. ; Selin, M. ; Vogt, U. ; Hertz, H. ; EsSer, D. ; Sipma, H. ; Luttmann, J. ; Höfer, M. ; Hoffmann, H. ; Yulin, S. ; Feigl, T. ; Rehbein, S. ; Guttmann, P. ; SchneiDer, G. ; Wiesemann, U. ; Wirtz, M. ; Diete, W.: Compact $\mathrm{x}$-ray microscope for the water window based on a high brightness laser plasma source. In: Optics Express 20 (2012), Juli, Nr. 16, S. 18362. http://dx.doi.org/10.1364/0E.20.018362. - DOI 10.1364/OE.20.018362

[20o] Legall, H. ; Stiel, H. ; Blobel, G. ; Seim, C. ; Baumann, J. ; Yulin, S. ; Esser, D. ; Höfer, M. ; Wiesemann, U. ; Wirtz, M. ; Schneider, G. ; Rehbein, S. ; Hertz, H.: A compact Laboratory Transmission X-ray Microscope for the water window. In: Journal of Physics: Conference Series 463 (2013), Oktober, S. o12013. http: //dx. doi .org/10. 1088/1742-6596/463/1/012013. - DOI 10.1088/1742-6596/463/1/012013

[201] Bertilson, M. ; Hofsten, O. von ; Thieme, J. ; Lindblom, M. ; Holmberg, A. ; TAKman, P. ; Vogt, U. ; Hertz, H.: First application experiments with the Stockholm compact soft $\mathrm{x}$-ray microscope. In: Journal of Physics: Conference Series 186 (2009), September, S. 012025. http://dx.doi .org/10.1088/1742-6596/186/1/012025. DOI 10.1088/1742-6596/186/1/012025

[202] H.Hertz ; Bertilson, M. ; Hofsten, O. von ; Gleber, S.-C. ; Sedlmair, J. ; Thieme, J.: Laboratory X-ray microscopy for high-resolution imaging of environmental colloid structure. In: Chemical Geology 329 (2012), November, S. 2631. http://dx.doi.org/10.1016/j.chemgeo.2011.07.012. - DOI 10.1016/j.chemgeo.2011.07.012

[203] Bertilson, M. ; Hofsten, O. von ; Vogt, U. ; Holmberg, A. ; Hertz, H.: High-resolution computed tomography with a compact soft x-ray microscope. In: Optics Express 17 (2009), Juni, Nr. 13, S. 11057. http://dx.doi.org/10.1364/0E.17.011057. - DOI 10.1364/OE.17.011057

[204] Bertilson, M. ; Hofsten, O. von ; Vogt, U. ; Holmberg, A. ; Christakou, A. ; Hertz, H.: Laboratory soft-x-ray microscope for cryotomography of biological specimens. In: Optics Letters 36 
(2011), Juli, Nr. 14, S. 2728. http://dx.doi.org/10.1364/0L.36. 002728. - DOI 10.1364/OL.36.002728

[205] Hertz, H. ; Hofsten, O. von ; Bertilson, M. ; Vogt, U. ; Holmberg, A. ; Reinspach, J. ; Martz, D. ; Selin, M. ; Christakou, A. ; Jerlström-Hultqvist, J. ; SvÄrd, S.: Laboratory cryo soft X-ray microscopy. In: Journal of Structural Biology 177 (2012), Februar, Nr. 2, S. 267-272. http://dx.doi .org/10 . 1016/j . j sb.2011. 11. 015. - DOI 10.1016/j.jsb.2011.11.015

[206] Müller, M. ; Mey, T. ; Niemeyer, J. ; Mann, K.: Table-top soft Xray microscope using laser-induced plasma from a pulsed gas jet. In: Optics Express 22 (2014), Sep, Nr. 19, 23489-23495342. http:// dx.doi.org/10.1364/0E.22.023489. - DOI 10.1364/OE.22.023489. - ISSN 1094-4087

[207] Müller, M. ; Mey, T. ; Niemeyer, J. ; Lorenz, M. ; Mann, K.: Table-top soft $\mathrm{X}$-ray microscopy with a laser-induced plasma source based on a pulsed gas-jet. In: AIP Conference Proceeding 1764 (2016), Nr. 1, S. 030003. http: //dx. doi .org/10. 1063/1.4961137. DOI 10.1063/1.4961137

[208] Wachulak, P. ; Torrisi, A. ; Bartnik, A. ; Adjei, D. ; Kostecki, J. ; Wegrzynski, L. ; Jarocki, R. ; Szczurek, M. ; Fiedorowicz, H.: Desktop water window microscope using a double-stream gas puff target source. In: Applied Physics B 118 (2015), Februar, Nr. 4, S. 573-578. http://dx. doi .org/10.1007/s00340-015-6044-x. DOI 10.1007/s00340-015-6044-X

[209] Wachulak, P. ; Torrisi, A. ; Nawaz, M. ; Bartnik, A. ; Adjei, D. ; Vondrová, Š ; Turňová, J. ; JanČareK, A. ; Limpouch, J. ; Vrbová, M. ; Fiedorowicz, H.: A Compact "Water Window" Microscope with 6o nm Spatial Resolution for Applications in Biology and Nanotechnology. In: Microscopy and Microanalysis 21 (2015), September, Nr. 05, S. 1214-1223. http://dx.doi.org/ 10.1017/S1431927615014750. - DOI 10.1017/S1431927615014750

[210] Bernhardt, M. ; Nicolas, J.-D. ; Osterhoff, M. ; Mittelstädt, H. ; Reuss, M. ; Harke, B. ; Wittmeier, A. ; Sprung, M. ; Köster, S. ; SAlditt, T.: Correlative microscopy approach for biology using X-ray holography, X-ray scanning diffraction and STED microscopy. In: Nature Communications 9 (2018), September, Nr. 1. http://dx.doi.org/10.1038/s41467-018-05885-z. - DOI 10.1038/s41467-018-05885-Z

[211] Friedrich, W. ; Knipping, P. ; LAue, M.: Interferenzerscheinungen bei Röntgenstrahlen. In: Annalen der Physik 346 (1913), Nr. 
10, S. 971-988. http://dx.doi.org/10. 1002/andp. 19133461004. DOI 10.1002/andp.19133461004

[212] Bragg, W. H. ; Bragg, W. L.: The Reflection of X-rays by Crystals. In: Proceedings of the Royal Society A 88 (1913), Juli, Nr. 605, S. 428-438. http://dx.doi.org/10.1098/rspa.1913.0040. - DOI 10.1098/rspa.1913.0040. - ISSN 1471-2946

[213] Sayers, D. E. ; Stern, E. A. ; Lyte, F. W.: New Technique for Investigating Noncrystalline Structures: Fourier Analysis of the Extended X-Ray-Absorption Fine Structure. In: Physical Review Letters 27 (1971), November, Nr. 18, S. 1204-1207. http://dx. doi.org/10.1103/PhysRevLett.27.1204. - DOI 10.1103/PhysRevLett.27.1204

[214] StÖHR, J.: NEXAFS Spectroscopy. Springer, 2003. - ISBN 3540544224

[215] Groot, F. de ; Kotani, A.: Core Level Spectroscopy of Solids (Advances in Condensed Matter Science). CRC Press, 2008. - ISBN 978-08493-9071-5

[216] Dewey, R. D. ; Mapes, R. S. ; Reynolds, T. W.: Handbook of Xray and Microprobe Data. Pergamon Press, 1969 (Analytical chemistry 9)

[217] Hüfner, S.: Photoelectron Spectroscopy. 3. Auflage. Springer, 2003. - ISBN 3-540-41802-4

[218] FRICKE, H.: The K-Characteristic Absorption Frequencies for the Chemical Elements Magnesium to Chromium. In: Physical Review 16 (1920), September, Nr. 3, S. 202. http://dx.doi.org/10.1103/ PhysRev.16.202. - DOI 10.1103/PhysRev.16.202

[219] Kapitel 15 The Historical Development of X-ray Absorption Fine Spectroscopy and of Its Applications to Materials Science. In: Pisano, R. (Hrsg.): A Bridge between Conceptual Frameworks. Springer Netherlands, 2015. - ISBN 978-94-017-9645-3, S. 275301

[220] Kortright, J. B. ; Awschalom, D. D. ; Stöhr, J. ; Bader, S. D. ; Idzerda, Y. U. ; PARKin, S. S. P. ; SChuller, I. K. ; SIEgmaNN, H.-C.: Research frontiers in magnetic materials at soft X-ray synchrotron radiation facilities. In: Journal of Magnetism and Magnetic Materials 207 (1999), November, Nr. 1-3, S. 744. http://dx.doi.org/10.1016/S0304-8853(99)00485- - . - DOI 10.1016/So304-8853(99)00485-O 
[221] Ade, H. ; Stoll, H.: Near-edge X-ray absorption fine-structure microscopy of organic and magnetic materials. In: Nature Materials 8 (2009), April, S. 281-290. http: //dx. doi . org/10 . 1038/nmat2399. - DOI 10.1038/nmat2399

[222] Abbate, M. ; Groot, F. de ; Fuggle, J. C. ; Ma, Y. J. ; Chen, C. T. ; Sette, F. ; Fujimori, A. ; ueda, Y. ; Kosuge, K.: Soft-Xray-absorption studies of the electronic-structure changes through the $\mathrm{VO}_{2}$ phase transition. In: Physical Review B 43 (1991), März, Nr. 9, S. 7263-7266. http://dx.doi.org/10.1103/PhysRevB.43.7263. - DOI 10.1103/PhysRevB.43.7263

[223] Schöll, A. ; Zou, Y. ; Schmidt, T. ; Fink, R. ; Uмbасн, E.: Energy calibration and intensity normalization in high-resolution NEXAFS spectroscopy. In: Journal of Electron Spectroscopy and Related Phenomena 129 (2003), März, Nr. 1, S. 1-8. http://dx. doi . org/10 . 1016/S0368-2048(03)00016-1. - DOI 10.1016/So3682048(03)00016-1

[224] Cavalleri, A. ; Rini, M. ; Chong, H. H. W. ; Fourmaux, S. ; Glover, T. E. ; Heimann, P. A. ; Kieffer, J. C. ; Schoenlein, R. W.: Band-Selective Measurements of Electron Dynamics in $\mathrm{VO}_{2}$ Using Femtosecond Near-Edge X-Ray Absorption. In: Physical Review Letters 95 (2005), August, Nr. 6, S. 067405. http://dx.doi. org/10.1103/PhysRevLett.95.067405. - DOI 10.1103/PhysRevLett.95.067405

[225] Roper, M. D. ; LaAn, G. van d. ; Flaherty, J. V. ; PadmoRE, H. A.: Soft x-ray spectroscopy in atmospheric pressure helium. In: Review of Scientific Instruments 63 (1992), Januar, Nr. 1, S. 1482-1485. http://dx.doi.org/10.1063/1.1143048. - DOI $10.1063 / 1.1143048$

[226] Wernet, P. ; Gavrila, G. ; Godehusen, K. ; Weniger, C. ; NibBERING, E. T. J. ; ElsAesser, T. ; EBERHARDT, W.: Ultrafast temperature jump in liquid water studied by a novel infrared pumpx-ray probe technique. In: Applied Physics A 92 (2008), August, Nr. 13, S. 511-516. http://dx. doi .org/10 . 1007/s00339-008-4726- 5. - DOI 10.1007/s00339-008-4726-5

[227] Nagasaka, M. ; Hatsui, T. ; Horigome, T. ; Hamamura, Y. ; Kosugi, N.: Development of a liquid flow cell to measure soft X-ray absorption in transmission mode: A test for liquid water. In: Journal of Electron Spectroscopy and Related Phenomena 177 (2010), März, Nr. 2-3, S. 130-134. http://dx. doi.org/10.1016/j .elspec . 2009.11.001. - DOI 10.1016/j.elspec.2009.11.001 
[228] Vogt, U. ; Wilhein, T. ; Stiel, H. ; Legall, H.: High resolution $\mathrm{x}$-ray absorption spectroscopy using a laser plasma radiation source. In: Review of Scientific Instruments 75 (2004), November, Nr. 11, S. 4606-46o9. http://dx.doi.org/10.1063/1.1808071. - DOI $10.1063 / 1.1808071$

[229] Mantouvalou, I. ; Witte, K. ; Martyanov, W. ; Jonas, A. ; Grötzsch, D. ; Streeck, C. ; Löchel, H. ; Rudolph, I. ; ERKO, A. ; Stiel, H. ; Kanngiesser, B.: Single shot near edge $x-$ ray absorption fine structure spectroscopy in the laboratory. In: Applied Physics Letters 108 (2016), April, Nr. 20, S. 201106. http: //dx.doi.org/10.1063/1.4951000. - DOI 10.1063/1.4951000

[230] KÜHL, F.-C.: Nah-Kanten-Absorptionsspektroskopie im weichen Röntgenbereich bei Atmosphärendruck, Hochschule für angewandte Wissenschaft und Kunst in Göttingen und Laser-Laboratorium Göttingen e.V., Bachelorarbeit, April 2013

[231] Grossmann, P. ; Rajkovic, I. ; Moré, R. ; Norpoth, J. ; TeCHERT, S. ; Jooss, C. ; MANN, K.: Time-resolved near-edge x-ray absorption fine structure spectroscopy on photo-induced phase transitions using a tabletop soft-x-ray spectrometer. In: Review of Scientific Instruments 83 (2012), Mai, Nr. 5, S. 053110. http: //dx.doi.org/10.1063/1.4718936. - DOI 10.1063/1.4718936

[232] Kühl, F.-C. ; Müller, M. ; Schellhorn, M. ; Mann, K. ; WieNEKE, S. ; Eusterhues, K.: Near-edge x-ray absorption fine structure spectroscopy at atmospheric pressure with a table-top laserinduced soft x-ray source. In: Journal of Vacuum Science $\mathcal{E}$ Technology A 34 (2016), Mai, Nr. 4, S. o41302. http: //dx. doi . org/10.1116/ 1.4950599. - DOI 10.1116/1.4950599

[233] Wachulak, P. ; Duda, M. ; Bartnik, A. ; Sarzyński, A. ; WęGRZYŃSKI, L. ; NoWAK, M. ; JANCAREK, A. ; FIEdorowicz, H.: Compact system for near edge X-ray fine structure (NEXAFS) spectroscopy using a laser-plasma light source. In: Optics Express 26 (2018), März, Nr. 7, S. 8260. http://dx.doi.org/10.1364/0E. 26. 008260. - DOI 10.1364/OE.26.008260

[234] Cousin, S. L. ; Silva, F. ; Teichmann, S. ; Hemmer, M. ; BuaDES, B. ; Biegert, J.: High-flux table-top soft x-ray source driven by sub-2-cycle, CEP stable, $1.85-\& \# x_{3} B C ; m$ 1-kHz pulses for carbon K-edge spectroscopy. In: Optics Letters 39 (2014), Nr. 18, S. 5383-5386. http://dx.doi.org/10.1364/0L.39.005383. - DOI 10.1364/OL.39.005383 
[235] Bressler, C. ; Chergui, M.: Ultrafast X-ray Absorption Spectroscopy. In: Chemical Reviews 100 (2004), März, Nr. 4, S. 1781-1812. http://dx.doi.org/10.1021/cr0206667. - DOI $10.1021 /$ cro206667

[236] Kita, T. ; Harada, T. ; Nakano, N. ; Kuroda, H.: Mechanically ruled aberration-corrected concave gratings for a flat-field grazing-incidence spectrograph. In: Applied Optics 22 (1983), Nr. 4, S. 512-513. http://dx.doi.org/10.1364/A0.22.000512. - DOI $10.1364 / A O .22 .000512$

[237] NIST Atomic Spectra Database Line Form. https://physics. nist.gov/PhysRefData/ASD/lines_form.html. - aufgerufen am 10.08.2017

[238] Nakano, N. ; Kuroda, H. ; Kita, T. ; Harada, T.: Development of a flat-field grazing-incidence XUV spectrometer and its application in picosecond XUV spectroscopy. In: Applied Optics 23 (1984), Juli, Nr. 14, S. 2386-2392. http://dx.doi.org/10.1364/A0. 23.002386. - DOI 10.1364/AO.23.002386

[239] Dinardo, M. E. ; Piazzalunga, A. ; Braicovich, L. ; Bisogni, V. ; Dallera, C. ; Giarda, K. ; Marcon, M. ; Tagliaferri, A. ; Ghiringhelli, G.: Gaining efficiency and resolution in soft Xray emission spectrometers thanks to directly illuminated CCD detectors. In: Nuclear Instruments and Methods in Physics Research Section A: Accelerators, Spectrometers, Detectors and Associated Equipment 570 (2006), Januar, Nr. 1, S. 176-181. http://dx. doi.org/10. 1016/j . nima. 2006.10.024. - DOI 10.1016/j.nima.2006.10.024

[240] Jordan-Sweet, J. ; Kovac, C. ; Goldberg, M. ; Morar, J.: Polymer/metal interfaces studied by carbon near-edge x-ray absorption fine structure spectroscopy. In: The Journal of Chemical Physics 89 (1988), August, Nr. 4, S. 2482-2489. http://dx.doi.org/10.1063/ 1.455042. - DOI 10.1063/1.455042

[241] KortüM, G. ; Müller, D.: Über die Gültigkeit des LambertBeerschen Gesetzes in Bromdampf. In: Zeitschrift für Naturforschung $A$ 1 (1946), August, Nr. 8. http://dx.doi.org/10.1515/ zna-1946-0804. - DOI 10.1515/zna-1946-0804

[242] Schaefer, C.: Über das ultrarote Absorptionsspektrum der Kohlensäure in seiner Abhängigkeit vom Druck. In: Annalen der Physik 321 (1905), Nr. 1, S. 93-105. http://dx. doi.org/10.1002/andp. 19053210108. - DOI 10.1002/andp.19053210108

[243] Todd, E. ; Sherman, D. ; Purton, J.: Surface oxidation of pyrite under ambient atmospheric and aqueous ( $\mathrm{pH}=2$ to 10) conditions: 
electronic structure and mineralogy from X-ray absorption spectroscopy. In: Geochimica et Cosmochimica Acta 67 (2003), März, Nr. 5, S. 881-893. http: //dx. doi . org/10. 1016/S0016- 7037 (02 ) 00957- 2. - DOI 10.1016/Soo16-7037(02)00957-2

[244] Andif1: Eigenes Werk, CC BY-SA 4.o. https: //commons .wikimedia. org/w/index . php?curid=58360992

[245] Brandt, F. ; Schäfer, T. ; Claret, F. ; Bosbach, D.: Heterogeneous formation of ferric oxide nanoparticles on chlorite surfaces studied by $\mathrm{x}$-ray absorption spectromicroscopy (STXM). In: Chemical Geology 329 (2012), November, Nr. 3, S. 4252. http://dx.doi.org/10.1016/j.chemgeo.2011.08.016. - DOI 10.1016/j.chemgeo.2011.08.016

[246] Gloter, A. ; Ingrin, J. ; Bouchet, D. ; Colliex, C.: Composition and orientation dependence of the $\mathrm{O} \mathrm{K}$ and $\mathrm{Fe} \mathrm{L}_{2,3}$ EELS fine structures in $\mathrm{Ca}_{2}\left(\mathrm{Al}_{x} \mathrm{Fe}_{1-\chi}\right)_{2} \mathrm{O}_{5}$. In: Physical Review B 61 (2000), Januar, Nr. 4, S. 2587-2594. http://dx. doi.org/10.1103/PhysRevB. 61.2587. - DOI 10.1103/PhysRevB.61.2587

[247] Wu, Z. ; Gota, S. ; Jollet, F. ; Pollak, M. ; Gautier-Soyer, M. ; Natoli, C.: Characterization of iron oxides by x-ray absorption at the oxygen $\mathrm{K}$ edge using a full multiple-scattering approach. In: Physical Review B 55 (1997), Januar, Nr. 4, S. 25702577. http://dx.doi.org/10.1103/PhysRevB.55.2570. - DOI 10.1103/PhysRevB.55.2570

[248] Thole, B. T. ; LAAN, G. van d.: Branching ratio in x-ray absorption spectroscopy. In: Physical Review B 38 (1988), August, Nr. 5, S. 3158-3171. http://dx.doi.org/10.1103/PhysRevB.38.3158. DOI 10.1103/PhysRevB.38.3158

[249] Parratt, L.: Electronic Band Structure of Solids by X-Ray Spectroscopy. In: Reviews of Modern Physics 31 (1959), Juli, Nr. 3, S. 616-645. http://dx.doi.org/10.1103/RevModPhys.31.616. - DOI 10.1103/RevModPhys.31.616

[250] Zaharieva, I. ; Chernev, P. ; Risch, M. ; Gerencser, L. ; Berggren, G. ; Shevchenko, D. ; Anderlund, M. ; Weng, T. ; Haumann, M. ; DAu, H.: Towards a comprehensive X-ray approach for studying the photosynthetic manganese complex XANES, $\mathrm{K} \alpha / \mathrm{K} \beta / \mathrm{K} \beta$-satellite emission lines, RIXS, and comparative computational approaches for selected model complexes. In: Journal of Physics: Conference Series 190 (2009), November, S. 012142. http://dx.doi.org/10.1088/1742-6596/190/1/012142. DOI 10.1088/1742-6596/190/1/012142 
[251] Naftel, S. ; Sham, T. ; Yiu, Y. ; Yates, B.: Calcium L-edge XANES study of some calcium compounds. In: Journal of Synchrotron Radiation 8 (2001), März, Nr. 2, S. 255-257. http: //dx. doi . org/10. 1107/S0909049500019555. - DOI 10.1107/So909049500019555

[252] Kapitel Calcium Chloride. In:Kemp, R. ; Kefgan, S.: Ullmann's Encyclopedia of Industrial Chemistry. Bd. 6. Wiley-VCH Verlag, 2000. - ISBN 9783527306732, 511-518

[253] Galasso, F. ; Kurti, N. (Hrsg.) ; Smoluchowski, R. (Hrsg.): Structure and Properties of Inorganic Solids. 1. Elsevier Science, 2016 (International Series of Monographs in Solid State Physics). http://www.ebook.de/de/product/23272869/francis_s_ galasso_structure_and_properties_of_inorganic_solids.html. - ISBN 9781483155418

[254] Picard-Lagnel, F. ; Poumellec, B. ; Cortes, R.: Study of amorphous thin films of $(\mathrm{Ti}, \mathrm{Nb}) \mathrm{O}_{2}$ solid solutions by $\mathrm{X}$-ray absorption at the Ti K edge. In: Journal of Physics and Chemistry of Solids 50 (1989), Januar, Nr. 12, S. 1211-1220. http://dx. doi.org/10.1016/ 0022 - 3697 ( 89 ) 90392 - 2. - DOI 10.1016/0022-3697(89)90392-2

[255] Lucarini, V. ; SaArinen, J. ; Peiponen, K.-E. ; Vartiainen, E.: Kramers-Kronig Relations in Optical Materials Research. Springer Berlin Heidelberg, 2006. - ISBN 978-3-540-27316-5

[256] Fuchs, O. ; Maier, F. ; Weinhardt, L. ; Weigand, M. ; Blum, M. ; Zharnikov, M. ; Denlinger, J. ; Grunze, M. ; Heske, C. ; Uмвасн, E.: A liquid flow cell to study the electronic structure of liquids with soft X-rays. In: Nuclear Instruments and Methods in Physics Research Section A: Accelerators, Spectrometers, Detectors and Associated Equipment 585 (2008), Februar, Nr. 3, S. 172177. http://dx.doi.org/10.1016/j.nima.2007.10.029. - DOI 10.1016/j.nima.2007.10.029

[257] Bora, D. ; Glans, P.-A. ; Pepper, J. ; Liu, Y.-S. ; Du, C. ; Wang, D. ; Guo, J.-H.: An ultra-high vacuum electrochemical flow cell for in situ/operando soft X-ray spectroscopy study. In: Review of Scientific Instruments 85 (2014), April, Nr. 4, S. 043106. http: //dx.doi.org/10.1063/1.4870795. - DOI 10.1063/1.4870795

[258] Prabu, V. ; Obst, M. ; Hosseinkhannazer, H. ; Reynolds, M. ; Rosendahl, S. ; WANG, J. ; Нiтснсоск, A.: Instrumentation for in situ flow electrochemical Scanning Transmission X-ray Microscopy (STXM). In: Review of Scientific Instruments 89 (2018), Juni, Nr. 6, S. o63702. http://dx.doi.org/10.1063/1.5023288. - DOI $10.1063 / 1.5023288$ 
[259] Hitchсоск, A. ; Morin, C. ; Zhang, X. ; Araki, T. ; Dynes, J. ; Stöver, H. ; Brash, J. ; LAwrence, J. ; Leppard, G.: Soft X-ray spectromicroscopy of biological and synthetic polymer systems. In: Journal of Electron Spectroscopy and Related Phenomena 144-147 (2005), Juni, S. 259-269. http://dx.doi.org/10.1016/j.elspec. 2005.01.279. - DOI 10.1016/j.elspec.2005.01.279

[26o] Rightor, E. ; Hitchсоск, A. ; Ade, H. ; Leapman, R. ; URQuhart, S. ; SMith, A. ; Mitchell, G. ; Fischer, D. ; Shin, H. ; Warwick, T.: Spectromicroscopy of Poly(ethylene terephthalate): Comparison of Spectra and Radiation Damage Rates in X-ray Absorption and Electron Energy Loss. In: The Journal of Physical Chemistry B 101 (1997), März, Nr. 11, S. 1950-1960. http: //dx.doi.org/10.1021/jp9622748. - DOI 10.1021/jp9622748

[261] Ade, H. ; Нiтснсоск, A.: NEXAFS microscopy and resonant scattering: Composition and orientation probed in real and reciprocal space. In: Polymer 49 (2008), Februar, Nr. 3, S. 643675. http://dx.doi.org/10.1016/j . polymer. 2007.10.030.-DOI 10.1016/j.polymer.2007.10.030

[262] Guttmann, P. ; Bittencourt, C. ; Rehbein, S. ; Umek, P. ; Ke, X. ; Tendeloo, G. V. ; Ewels, C. ; Schneider, G.: Nanoscale spectroscopy with polarized X-rays by NEXAFS-TXM. In: Nature Photonics 6 (2011), November, Nr. 1, S. 25-29. http: //dx. doi.org/ 10. 1038/NPHOTON . 2011 . 268. - DOI 10.1038/NPHOTON.2011.268

[263] Prietzel, J. ; Müller, S. ; Kögel-Knabner, I. ; Thieme, J. ; JAYE, C. ; FisCHER, D.: Comparison of soil organic carbon speciation using C NEXAFS and CPMAS 13 C NMR spectroscopy. In: Science of The Total Environment 628-629 (2018), Juli, S. 906-918. http://dx.doi.org/10.1016/j.scitotenv.2018.02.121. - DOI 10.1016/j.scitotenv.2018.02.121

[264] Johansson, G. ; TyliszczaK, T. ; Mitchell, G. ; Keefe, M. ; HitCHсOCK, A.: Three-dimensional chemical mapping by scanning transmission X-ray spectromicroscopy. In: Journal of Synchrotron Radiation 14 (2007), August, Nr. 5, S. 395-402. http : //dx. doi . org/ 10.1107/S0909049507029962. - DOI 10.1107/So909049507029962

[265] Овsт, M. ; Wang, J. ; Нітснсоск, A. P.: Soft X-ray spectrotomography study of cyanobacterial biomineral nucleation. In: Geobiology 7 (2009), Dezember, Nr. 5, S. 577-591. http://dx.doi. org/10.1111/j.1472-4669.2009.00221.x. - DOI 10.1111/j.14724669.2009.00221.x 
[266] Hitchсоск, A. ; Dynes, J. ; Johansson, G. ; Wang, J. ; ВотTON, G.: Comparison of NEXAFS microscopy and TEM-EELS for studies of soft matter. In: Micron 39 (2008), April, Nr. 3, S. 311319. http://dx.doi.org/10.1016/j.micron.2007.09.008. - DOI 10.1016/j.micron.2007.09.008

[267] Lim, J. ; Li, Y. ; Alsem, D. ; So, H. ; Lee, S. ; P. ; Cogswell, D. ; LiU, X. ; Jin, N. ; Yu, Y.-S. ; Salmon, N. ; Shapiro, D. ; Bazant, M. ; TrliszczaK, T. ; ChueH, W.: Origin and hysteresis of lithium compositional spatiodynamics within battery primary particles. In: Science 353 (2016), August, Nr. 6299, S. 566-571. http://dx.doi. org/10.1126/science. aaf4914. - DOI 10.1126/science.aaf4914

[268] Yu, Y.-S. ; Farmand, M. ; Kim, C. ; Liu, Y. ; Grey, C. ; Strobridge, F. ; Tyliszczak, T. ; Celestre, R. ; Denes, P. ; Joseph, J. ; Krishnan, H. ; Maia, F. ; Kilcoyne, D. ; Marchesini, S. ; Leite, T. ; Warwick, T. ; Padmore, H. ; Cabana, J. ; Shapiro, D.: Three-dimensional localization of nanoscale battery reactions using soft X-ray tomography. In: Nature Communications 9 (2018), März, Nr. 1. http://dx. doi .org/10.1038/s41467-018-03401-x. DOI 10.1038/s41467-018-03401-x

[269] Farmand, M. ; Celestre, R. ; Denes, P. ; Kilcoyne, D. ; MarChesini, S. ; PAdmore, H. ; Tyliszczak, T. ; Warwick, T. ; ShI, X. ; Lee, J. ; Yu, Y.-S. ; Cabana, J. ; Joseph, J. ; Krishnan, H. ; Perciano, T. ; Maia, F. ; Shapiro, D.: Near-edge X-ray refraction fine structure microscopy. In: Applied Physics Letters 110 (2017), Februar, Nr. 6, S. o63101. http: //dx. doi.org/10.1063/1. 4975377. - DOI 10.1063/1.4975377

[270] Watts, B. ; AdE, H.: NEXAFS imaging of synthetic organic materials. In: Materials Today 15 (2012), April, Nr. 4, S. 148157. http : //dx. doi.org/10.1016/S1369-7021 (12) 70068-8. - DOI 10.1016/S1369-7021(12)70068-8 
Einige der in dieser Arbeit beschriebenen Ideen und gezeigten Abbildungen finden sich so oder so ähnlich in folgenden Publikationen:

M. Müller, F.-C. Kühl, P. Großmann, P. Vrba und K. Mann

"Emission properties of ns and ps laser-induced soft $\mathrm{x}$-ray sources using pulsed gas jets"

Optics Express 21(10), 12831 (2013)

M. Müller, T. Mey, J. Niemeyer und K. Mann

"Table-top soft x-ray microscope using laser-induced plasma from a pulsed gas jet"

Optics Express 22(19), 023489 (2014)

F.-C. Kühl, M. Müller, M. Schellhorn, K. Mann, S. Wieneke und K. Eusterhues

"Near-edge x-ray absorption fine structure spectroscopy at atmospheric pressure with a table-top laser-induced soft x-ray source"

Journal of Vacuum Science and Technology A 34, 041302 (2016)

M. Müller, T. Mey, J. Niemeyer, M. Lorenz und K. Mann

"Table-top soft $\mathrm{x}$-ray microscopy with a laser-induced plasma source based on a pulsed gas jet"

AIP Conference Proceedings 1764, 030003 (2016) 
Weitere wissenschaftliche Beiträge:

A. J. Corso, P. Zuppella, F. Barkusky, K. Mann, M. Müller, P. Nicolosi, M. Nardello und M. G. Pelizzo

"Damage of multilayer optics with varying capping layers induced by focused extreme ultraviolet beam"

Journal of Applied Physics 108, 203106 (2013)

C. Liberatore, K. Mann, M. Müller, L. Pina, L. Juha, L. Vysin, J. Rocca,

A. Endo und T. Mocek

"Short-wavelength ablation of polymers in the high-fluence regime"

Physica Scripta 2014, T161 (2014)

C. Liberatore, K. Mann, M. Müller, L. Pina, L. Juha, J. Rocca, A. Endo und T. Mocek

"EUV ablation of organic polymers at a high fluence"

High Power Laser Science and Engineering 2, e26 (2014)

M. Nikl, V. Babin, J. Pejchal, V.V. Laguta, M. Buryi, J.A. Mares,

K. Kamada, S. Kurosawa, A. Yoshikawa, D. Panek, P. Bruza,

K. Mann und M. Müller

"The stable $\mathrm{Ce}^{4+}$ center: a new tool to optimize Ce-doped

oxide scintillators"

IEEE Transactions on Nuclear Science 63(2), 433 (2016)

F. Döring, H. Ulrichs, S. Pagel, M. Müller, M. Mansurova, M. Müller,

C. Eberl, T. Erichsen, D. Huebner, P. Vana, K. Mann, M. Münzenberg und H.-U. Krebs

"Confinement of phonon propagation in laser deposited Tungsten/Polycarbonate multilayers"

New Journal of Physics 18, 111001 (2016)

P. Vrba, M. Vrbova, M. Müller, K. Mann. D. Panek und T. Parkman "Picosecond laser krypton plasma emission in water window spectral range" Physics of Plasmas 24, 123301 (2017) 
KONFERENZBEITR R̈GE

Teile dieser Arbeit wurden auf folgenden Konferenzen präsentiert:

DPG-Frühjahrstagung - Vortrag, Berlin, Deutschland (2014)

$2^{\text {nd }}$ BOX Workshop - Vortrag, Kladno, Tschechien (2014)

COST Action MP1203 Advanced X-ray spatial and temporal metrology - Poster, Dubrovnik, Kroatien (2014)

ICXOM23 - Vortrag, Brookhaven National Laboratory, USA (2015)

International Symposium Biological Dynamics from Microscopic to Mesoscopic Scales - Vortrag und Poster, Grimma, Deutschland (2016)

304 ${ }^{\text {th }}$ PTB-Seminar VUV and EUV Metrology - Vortrag, Berlin, Deutschland (2017) 
Während meiner Promotion haben mich viele Menschen begleitet, ohne die diese Arbeit nicht erfolgreich gewesen wäre. Dafür möchte ich mich ganz herzlich bei allen bedanken.

Mein besonderer Dank gilt Dr. Klaus Mann. Er hat mir die Möglichkeit gegeben, meine Dissertation am Laser-Laboratorium Göttingen e.V. anzufertigen. Dabei hat er mich die gesamte Zeit ausgezeichnet betreut und mit sehr viel Engagement unterstützt. Besonders gefreut habe ich mich über sein großes Vertrauen, das es mir ermöglichte, meine Promotion selbstständig durchzuführen, entscheidend mitzugestalten und eigene Ideen zu verwirklichen. Vielen, vielen Dank für die tolle Zusammenarbeit, die mich fachlich und persönlich sehr bereichert hat!

Prof. Dr. Tim Salditt danke ich sehr herzlich für die Übernahme des Erstgutachtens und damit verbunden die Einbindung in das spannende wissenschaftliche Umfeld des Sonderforschungsbereichs 755 „Nanoscale Photonic Imaging". Seine Unterstützung und seine Fähigkeit zu begeistern, haben sehr zum Gelingen dieser Arbeit beigetragen.

Bei PD Dr. Alexander Egner, PD Dr. Jürgen Niemeyer, Prof. Dr. Arnulf Quadt und Prof. Dr. Claus Ropers bedanke ich mich für ihren Einsatz in der Prüfungskommission. Jürgen Niemeyer möchte ich darüber hinaus für die vielen hilfreichen Diskussionen und Erläuterungen z. B. zur Analyse der NEXAFS-Spektren danken.

Ich bedanke mich bei allen jetzigen und ehemaligen Kolleginnen und Kollegen des LLGs und speziell der Abteilung „Optik / Kurze Wellenlängen“ für die große Hilfsbereitschaft und angenehme Arbeitsatmosphäre. Besonders erwähnen möchte ich dabei Uwe Lampe und Maik Lübbecke. Bei Uwe Lampe bedanke ich mich für die hervorragende Unterstützung bei den unzähligen Bestellungen sowie die vielen spannenden Gespräche und Diskussionen über Fußball und Sport im Allgemeinen. Maik Lübbecke gebührt mein Dank nicht nur für die Hilfe bei Elektronikproblemen, sondern auch für die tolle und lustige Zeit im Büro und die spannenden Kickerpartien.

Die Promotion wurde im Rahmen des SFB 755 im Projekt Co4 "Soft x-ray spectro-microscopy with a lab-scale source" von der Deutschen Forschungsgemeinschaft finanziert. Für die Förderung wird gedankt. Für die Unterstützung bei administrativen Angelegenheiten im SFB 755 und für die herausragende Organisation verschiedenster Veranstaltungen möchte ich mich zudem herzlich bei Eva Hetzel und Christine WilkeFeist bedanken. 
Ein großes Dankeschön gilt auch meinen Freundinnen und Freunden. Ihnen ist es zu verdanken, dass ich mich vom ersten Tag an bis heute in Göttingen wohlfühle. Die gemeinsame Zeit mit vielen Doppelkopfrunden, Ausflügen, Mensatreffen ... werde ich nie vergessen.

Meiner Familie bin ich von ganzem Herzen dankbar für die grenzenlose Unterstützung und den bedingungslosen Rückhalt in all den Jahren. Ohne sie wäre all das undenkbar gewesen. Danke, dass ihr immer für mich da seid! 
Person

Name Matthias Müller

Geburtsdatum 21.04.1987

Geburtsort Karl-Marx-Stadt (heute Chemnitz)

Staatsangehörigkeit deutsch

Wissenschaftlicher Werdegang

06/2006 Abitur am Albert-Einstein-Gymnasium, Buchholz i.d. Nordheide

10/2007 - 09/2010 Bachelorstudium Physik

Georg-August-Universität Göttingen

10/2010 - 09/2012 Masterstudium Physik

Georg-August Universität Göttingen \& Laser-Laboratorium Göttingen e.V.

09/2012 Master of Science in Physik (M. Sc.)

Masterarbeit: Stabilität und Übertragungseigenschaften von Optiken für den extremen UV- und weichen Röntgenbereich

seit 11/2012 Wissenschaftlicher Mitarbeiter am

Laser-Laboratorium Göttingen e.V.

11/2012 - 10/2018 Promotionsstudium Physik

Georg-August Universität Göttingen 